\title{
Unbiased evaluation and development of Green's-function-based density-matrix functionals
}

\author{
Dissertation \\ zur Erlangung des mathematisch-naturwissenschaftlichen Doktorgrades \\ "Doctor rerum naturalium" \\ der Georg-August-Universität Göttingen \\ im Promotionsprogramm ProPhys \\ der Georg-August University School of Science (GAUSS)
}

vorgelegt von

\section{Ebad Kamil}

aus Barabanki, Indien

Göttingen, 2016 


\section{Betreuungsausschuss}

Prof. Dr. Peter E. Blöchl, Institut für Theoretische Physik, Technische Universität Clausthal

Prof. Dr. Stefan Kehrein, Institut für Theoretische Physik, Georg-August-Universität Göttingen

\section{Mitglieder der Prüfungskommission}

Referent: Prof. Dr. Peter E. Blöchl, Institut für Theoretische Physik,

Technische Universität Clausthal

Korreferent: Prof. Dr. Stefan Kehrein, Institut für Theoretische Physik, Georg-August-Universität Göttingen

\section{Weitere Mitglieder der Prüfungskommission}

Priv.-Doz. Dr. Salvatore R. Manmana, Institut für Theoretische Physik,

Georg-August-Universität Göttingen

apl. Prof. Dr. Michael Seibt,

IV. Physikalisches Institut,

Georg-August-Universität Göttingen

Prof. Dr. Marcus Müller,

Institut für Theoretische Physik,

Georg-August-Universität Göttingen

Prof. Dr. Stefan Mathias,

I. Physikalisches Institut,

Georg-August-Universität Göttingen

Tag der mündlichen Prüfung: 6 July, 2016 
Speak, for your lips are yet free;

Speak, for your tongue is still your own;

Your lissom body yours alone;

Speak, your life is still your own.

Look into the blacksmith's forge:

The flame blazes, the iron's red;

Locks unfasten open-mouthed,

Every chain's link springing wide.

Speak, a little time suffices

Before the tongue, the body die.

Speak, the truth is still alive;

Speak, say what you have to say $\mathbb{1}^{1}$

— Faiz Ahmad Faiz (1911-1984)

Dedicated to my family, for love and patience.

To Thomas and Peter, for opportunity and support.

\footnotetext{
${ }^{1}$ The original version is in Urdu. The english version is obtained from a website which cites Ms. Yasmin Hosain as the translator.
} 



\section{Contents}

Page

$\begin{array}{lll}1 & \text { Introduction } & 1\end{array}$

2 Reduced density matrix functional theory 5

2.1 General solid state system . . . . . . . . . . . . . . . . . . 5

2.2 Born-Oppenheimer Approximation . . . . . . . . . . . . . . . 6

2.3 Solid state system in second quantization . . . . . . . . . . . . . . . 8

2.3 .1 Local spin orbitals $\ldots \ldots \ldots \ldots$. . . . . . . . . . . . 8

2.3 .2 Projector states . . . . . . . . . . . . . . . . . . . . . . . . . . . 9

2.3.3 Hamiltonian in second quantization . . . . . . . . . . . . . . . . 9

2.4 Grand-canonical potential . . . . . . . . . . . . . . . . . . . . . . . . . . . . . 11

2.5 Important quantities . . . . . . . . . . . . . . . . . . . . . . . . . . . . . . . . . . . . . . . . . . . .

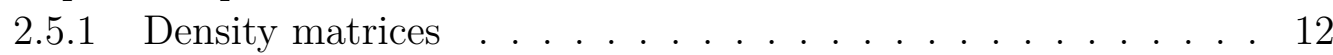

2.5 .2 Green's function and self energy . . . . . . . . . . . . . . . . 15

2.5.3 Lehmann representation of the Green's function . . . . . . . . . 17

2.5.4 Relation between one particle density matrix and the Green's

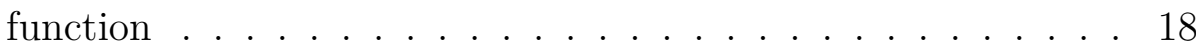

2.5.5 Relation between self energy and the interaction energy . . . . 19

2.6 Gilbert theorem and the reduced density matrix functional . . . . . . 20

2.6 .1 Wave-function based approach . . . . . . . . . . . . . . 21

2.6 .2 Green's function based method . . . . . . . . . . . . . . . . 23

2.6 .3 Parametrized density matrix functionals . . . . . . . . . . . 27

2.7 Test systems $\ldots \ldots \ldots \ldots \ldots \ldots \ldots \ldots$

2.7 .1 Hubbard Dimer and the exact results . . . . . . . . . . . . . 36

$2.7 .2 \quad$ Exact energy and density matrix . . . . . . . . . . . . . . . . . . . 37

2.7 .3 Natural orbitals and occupations . . . . . . . . . . . . . . . 39

2.7 .4 Correlations . . . . . . . . . . . . . . . . . . . . 44

\begin{tabular}{lll}
\hline 3 & Numerical optimization scheme & 43
\end{tabular}

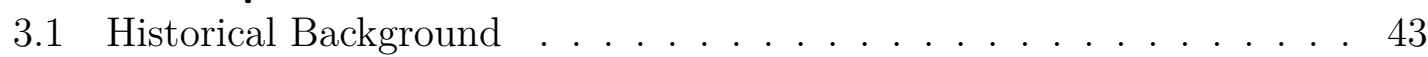

3.2 Numerical simulation . . . . . . . . . . . . . . . . . . . . . . . . 44

3.2 .1 Time evolution . . . . . . . . . . . . . . . . . . . . . . . . . . . . . . . 48

3.3 Constraints $\ldots \ldots \ldots \ldots \ldots$

3.3 .1 Wave function constraint . . . . . . . . . . . . . . . . . . 48 
$3.3 .2 \quad$ Occupation constraint . . . . . . . . . . . . . . . . 49

3.4 Convergence, stability and accuracy . . . . . . . . . . . . . . . . . . . 49

$3.4 .1 \quad$ Accuracy and Stability of Verlet Algorithm. . . . . . . . . . . 49

3.4.2 Convergence . . . . . . . . . . . . . . . . . 50 50

4 Parametrized density matrix functionals 53

4.1 Grand canonical potential and the density matrix functional . . . . . 53

4.2 Construction of density-matrix functionals . . . . . . . . . . . 54

4.3 Optimization scheme for the evaluation of the grand canonical potential 55

4.4 Performance of density-matrix functionals $\ldots \ldots \ldots \ldots 6$

$4.4 .1 \quad$ Hartree-Fock approximation . . . . . . . . . . . . . . . . . 56

4.4 .2 Müller's functional . . . . . . . . . . . . . . . . . . . . . . . . 59

4.5 Power functional . . . . . . . . . . . . . . . . . . . . . . . . . . . 64

4.6 Beyond half-filling . . . . . . . . . . . . . . . . . . . . . 71

4.7 Beyond the dimer . . . . . . . . . . . . . . . . . . . . . . . . . 74

4.8 Summary $\ldots \ldots \ldots \ldots$. . . . . . . . . . . . . . . . . . . . 75

5 Density matrix functionals derived from Green's function 81

5.1 Framework for construction of approximate scheme . . . . . . . . . . 81

5.2 Approximation for Luttinger Ward functional $\left.\Phi_{\beta}^{L W}[\boldsymbol{G}[\boldsymbol{\rho}], \hat{W}]\right]$. . . . . 83

5.2 .1 Hartree-Fock approximation $\ldots \ldots$. . . . . . . . 83

5.2 .2 Second order diagrams . . . . . . . . . . . . . . . . . . . 85

$5.2 .3 \quad$ Higher order diagrams . . . . . . . . . . . . . . . . . . 88

5.2 .4 Regularization . . . . . . . . . . . . . . . . . . . . . . 90

5.3 Model Green's function . . . . . . . . . . . . . . . . . . . . . . . . . 92

$5.3 .1 \quad$ Single-pole approximation . . . . . . . . . . . . . . . . . 93

5.3 .2 Two-pole approximation . . . . . . . . . . . . . . . . . . . . 94

5.4 Performance of the density matrix functional . . . . . . . . . . . . . . 98

$5.4 .1 \quad$ Approximate density matrix functional . . . . . . . . . . . . . 98

5.4 .2 Results . . . . . . . . . . . . . . . . . . . . 100

5.4 .3 Consistent way to fix $\Delta \ldots \ldots \ldots$. . . . . . . . . 103

5.5 Summary . . . . . . . . . . . . . . . . . . . . . 104

6 Conclusion and Outlook 107

\begin{tabular}{ll}
\hline Appendices & 111
\end{tabular}

\begin{tabular}{|lr}
\hline A Evaluation of derivative matrix $D$ & 113
\end{tabular}

\begin{tabular}{ll}
\hline B Unconstrained Optimization & 115
\end{tabular}

C Grand potential and the Luttinger Ward functional $\quad \mathbf{1 1 9}$

C.1 Rules to evaluate the diagrams . . . . . . . . . . . . . . . . 120 
C.2 Symmetry factors of the diagrams . . . . . . . . . . . . . . . 122

C.3 Linked cluster theorem . . . . . . . . . . . . . . . . . . . . . . . . 123

C.4 Luttinger Ward functional $\left.\Phi^{\tilde{W}}[\boldsymbol{G}]\right] \ldots \ldots \ldots \ldots$

\begin{tabular}{ll}
\hline Bibliography & 127
\end{tabular} 



\section{Introduction}

The general solid-state system is completely determined by its constituent particles and their respective interactions. The electrons and the nuclei are the constituents which interact through electro-static Coulomb interaction. Nuclei on the other hand are made of neutrons and protons which interact via weak and strong nuclear forces. However these short-range nuclear interactions do not contribute to the macroscopic properties of the solid-state system. The gravitational interactions among the constituent particles is also neglected because of their small mass.

With the knowledge of all the relevant interactions, the stage is set to solve the governing equation of the quantum systems i.e. the time-dependent Schrödinger equation. In quantum mechanics, the system is described by a many-particle wavefunction which is the solution of the Schrödinger equation. The biggest hurdle in the evaluation of the many-particle wave-function, is the exponential growth of the Hilbert space with the number of constituent particles. The gigantic Hilbert space in almost all the cases of an interacting system, makes it impossible to attempt for an exact solution.

To overcome the problem of an exponential growth of the Hilbert space with the system size, one first proceed towards separating the degrees of freedom involved, which in this case are the electrons and the nuclei. Due to an enormous difference between the mass of an electron and the nuclei, Born-Oppenheimer treatment [1] is the first step towards separating the dynamics of nuclei and the electrons. It allows the dynamics of the nuclei to be studied separately in the presence of a potential field (Born-Oppenheimer surface) which can be approximated by an empirical interaction (Classical molecular dynamics) or evaluated from state of the art electronic structure calculations (ab-initio molecular dynamics).

The second branch of approximation comes in during the treatment of the electronic degrees of freedom. The exact treatment of an interacting electron system is again an impossible task due to a formidable Hilbert space. The electronic manybody wave-function which depends parametrically on the nuclear degrees of freedom can not be exactly evaluated in almost all the cases of an interacting quantum system. The full-information of the many-particle wave-function is however inordinate. The valuable physical properties of the electronic system is mostly obtained from the one-body (kinetic energy, magnetization etc) or the two-body (electron-interaction, magnetic susceptibility etc.) operators. To this end, the concept of density-matrices, specifically the one-particle and two-particle reduced density-matrices, single particle Green's function, self-energy etc. is paramount. 
In solid-state physics, formalisms like density-functional theory (DFT) [2, 3, 4, 5], reduced density-matrix functional theory [6, 7], many-body perturbation theory [8, 9], dynamical mean-field theory [10, 11, 12], etc, are used to study the electronic properties of the system.

Ab-initio calculations are dominated by density functional theory (DFT), which provides an efficient and accurate description of the electronic structure for most materials [13]. In the DFT framework, an interacting electron system is rigorously mapped to a system of non-interacting particles (Kohn-Sham system) with an effective potential that reproduces the density of the original system. The effective potential in turn depends on the density and therefore the Kohn-Sham set of equations need to be solved self-consistently. The entire complexity of the many-body interactions is clubbed into a density-functional or the exchange-correlation energy. The first density-functional which were used in practice were based on local density approximation (LDA). It worked surprisingly well for real materials with weak correlations [13]. LDA was however inept for most applications in chemistry due to its deficiency to describe bond breaking correctly. The situation was considerably improved by the introduction of generalized gradient approximations (GGA) [14, 15] which reproduces the bond energies to an accuracy of few tenth of an electron volt. The most widely used GGA functional is the Perdew-Burke-Ernzerhof (PBE) functional [16, 17]. Compared to GGA functionals, another class of density-functionals which provides better estimates of binding energies, band gaps etc, are the Hybrid functionals [18, 19, 20, 21, 22, 23]. The hybrid-functionals replace a part of exchangecorrelation energy by the exact exchange. The most widely used hybrid functional are B3YLP [24] and PBE0 [25, 26].

For materials with strong correlations, however, many of the available density functionals yield poor results [27, 28]. Most well known is the case of transition metal oxides, for which most density functionals produce a qualitatively incorrect description [29]. There is a quest to improve the description by borrowing from methods specifically designed for strongly correlated materials. One of the method which is widely used to study strongly correlated electron system in the thermodynamic limit is the dynamical mean-field theory (DMFT). DMFT treats the local correlations explicitly by mapping a complicated lattice model to an impurity system with an effective bath which is determined self-consistently. One of the biggest success for DMFT was the correct description of the phase-transition between a metal and a Mott insulator [30, 12, 31]. For calculations pertaining to real materials, approaches like DFT+DMFT [12, 32, 33, 34, 35] are commonly used. Other methods which are also quite extensively used to study real materials are LDA $+\mathrm{U}[36$, 37, 38, 39] and DFT-plus-Gutzwiller approximation[40, 41, 42, 43]. The guiding idea behind these approaches is to merge density functional theory with methods developed for the study of strong correlations for model Hamiltonians such as the Hubbard model [44, 45, 46. These hybrid methods are immensely successfull in the advance- 
ment of our understanding behind the physics of Mott transition, structural phase diagrams, optical conductivity, transport etc. in the strongly correlated real materials.

We consider reduced density-matrix functional theory (rDMFT) [6, 7] to be a useful framework for a rigorous formulation of such hybrid theories 47, 48. Reduced density-matrix functional theory can be viewed as a relative of DFT, which emphasizes orbital occupations rather than the density as basic variable. Such a description seems to be natural for correlated materials, because the latter are dominated by orbital physics. In 1975, Gilbert [6] proved that there exist a one-to-one mapping between non-degenerate ground-state wave-function and the corresponding one-particle reduced density matrix. This lead to construction of the ground-state properties of the electronic system as a functional of one-particle reduced density matrix. Further, constrained minimization in the space of N-representable oneparticle reduced density matrices leads to the state with the minimum energy or the ground-state. The complexity of the explicit many-body effects are concealed in an object called the reduced density-matrix functional.

The link from rDMFT to many-particle wave functions has been established by Levy's constrained-search algorithm [7] on the one hand. The link to many-body perturbation theory and Green's function, on the other hand, has been provided recently [48] via the Luttinger-Ward functional [49]. However until half a decade ago, the development in the field of rDMFT proceeded analogously to the development of DFT. In order to avoid the full complexity of an explicit many-body description, most density-matrix functionals are not extracted from the exact expressions [7, 48. Rather, one proceeds analogously to the development of density functionals, namely by searching models for the density-matrix functional, that capture the most essential physical effects while having an algebraic dependence on the density matrix. Among such models, the most prominent ones are the Müller functional[50, 51, Goedecker-Umrigar functional [52], Sharma functional [53] and Marques-Lathiotakis functional [54].

One of the major arguments in favor of density-matrix functionals is that one of the most simple functionals, the Müller functional [50], seems to provide a correct description of the bond-dissociation problem, for which common density functionals fail [55]. Despite the successes, the common density-matrix functionals also reproduce a number of features in a qualitatively incorrect manner [56]. Several such discrepancies of the standard state of the art density-matrix functionals are discussed in details in this thesis.

The objective of this thesis is to study the performance of the class of commonly used model density-matrix functionals and to set the stage for the desire to develop the framework for an approximate scheme to evaluate new class of functionals from exact Green's function based method [48. To this end the thesis is organized as follows. 
Chapter 2 is the introductory chapter where we discuss the basic concepts of the rDMFT. We start with introducing the model Hamiltonian and the important static and dynamical objects like the density matrices, Green's function and the self-energy etc. The notion of density-matrix functional and its evaluation via three different methods namely the wave-function based, Green's function based and the empirical approaches, are discussed towards the later half of the chapter. We end the chapter by discussing some of the exact results for the model benchmark systems.

Chapter 3 is devoted towards the numerical methodology of searching for the ground-state. The optimization in the space of N-representable one-particle reduced density matrices, is done in Car-Parinello [57, 58] spirit. Starting with the historical background of the minimization algorithm, we discuss its practical implementation suitable for our purpose. The treatment of the constraints and the issues related to the convergence and stability of the algorithm are discussed in details.

In Chapter 4, we analyze the quality of existing state of the art parametrized density-matrix functionals. We discuss in particular the Hartree-Fock, Müller [50, 51] and the power functionals [53. The performance of these functionals are benchmarked on exactly solvable system like the Hubbard dimer. Several discrepancies like the tendency of Müller functional to produce infinite magnetic polarizability at zero temperature, abrupt artifical transition to anti-ferromagnetic states in case of power functionals etc are highlighted. The problems are also shown to persist in larger systems like the Hubbard chains or the rings.

Chapter 5, is devoted towards the development of new class of functionals based on the Green's function based methods [48]. We develop a new framework to avoid the complicated stationary point search in the space of Green's function and self-energy during the evaluation the density-matrix functional. The goal is to provide an algebraic form of the density-matrix functional in terms of Luttinger-Ward functional. Using the standard perturbation theory in the interaction strength, we aim to build a controlled scheme towards the construction of the density-matrix functional. 


\section{Reduced density matrix functional theory}

\subsection{General solid state system}

The full Hamiltonian which governs the static and dynamic properties of the solid state system can be decomposed as,

$$
\hat{\mathcal{H}}=\hat{T}_{e}+\hat{T}_{n}+\hat{W}_{e-e}+\hat{W}_{n-n}+\hat{W}_{e-n}
$$

where $\hat{T}_{e}$ and $\hat{T}_{n}$ are the kinetic energy operators for the electrons and the nuclei respectively. $\hat{W}_{e-e}$ is the Coulomb repulsion between the electrons, $\hat{W}_{n-n}$ is the electro-static interaction between the nuclei and $\hat{W}_{e-n}$ is the electro-static interactions between the electrons and the nuclei.

The operators in Eq. 2.1 can be written in their full glory as

$$
\begin{aligned}
\hat{T}_{e} & =\sum_{i=1}^{N}-\frac{\hbar^{2}}{2 m_{e}} \nabla_{i}^{2} \quad \text { where } \nabla_{i}^{2}=\frac{\partial^{2}}{\partial r_{i}^{2}} \\
\hat{T}_{n} & =\sum_{i=1}^{M}-\frac{\hbar^{2}}{2 M_{i}} \nabla_{i}^{2} \quad \text { where } \nabla_{i}^{2}=\frac{\partial^{2}}{\partial R_{i}^{2}} \\
\hat{W}_{e-e} & =\sum_{i \neq j} \frac{e^{2}}{4 \pi \epsilon_{0}\left|\vec{r}_{i}-\vec{r}_{j}\right|^{2}} \\
\hat{W}_{n-n} & =\sum_{i=1}^{M} \sum_{j=1}^{M} \frac{Z_{i} Z_{j} e^{2}}{4 \pi \epsilon_{0}\left|\vec{R}_{i}-\vec{R}_{j}\right|^{2}} \\
\hat{W}_{e-n} & =-\sum_{i=1}^{N} \sum_{j=1}^{M} \frac{Z_{j} e^{2}}{4 \pi \epsilon_{0}\left|\vec{r}_{i}-\vec{R}_{j}\right|^{2}}
\end{aligned}
$$

where $e$ and $m_{e}$ are the unit charge and the mass of an electron respectively. $Z_{i}$ and $M_{i}$ are the atomic number and the mass of $i$-th nucleus respectively. $\epsilon_{0}$ is the electric permitivity of the free space.

The dynamics of the constituent particles is governed by the celebrated time- 
dependent Schrödinger equation, which has the form,

$$
i \hbar \frac{\partial}{\partial t}|\Phi\rangle=\hat{\mathcal{H}}|\Phi\rangle
$$

where the ket $|\Phi\rangle$ describes the many-body state of the electrons and the nuclei. For a solid state system comprising of $\mathrm{N}$ electrons and $\mathrm{M}$ nuclei, the many-particle wave-function has the following form,

$$
\Phi\left(\vec{x}_{1}, \vec{x}_{2}, \ldots \vec{x}_{N}, \vec{R}_{1}, \vec{R}_{2}, \ldots \vec{R}_{M}\right)
$$

where $\vec{x}_{i}=\left(\vec{r}_{i}, \sigma_{i}\right)$ is a composite index which labels an electron at position $\vec{r}_{i}$ with a spin $\sigma_{i}$. The spin index $\sigma$ for the electrons can take one of the two values from the set $\{\uparrow, \downarrow\}$. The position vector of the $i$-th nucleus is labeled as $\vec{R}_{i}$.

The large number of the degrees of freedom renders an exact solution for the time-dependent Schrödinger equation impossible in almost all cases. The progress of theoretical physics relies on proposing controlled approximations which simplifies the calculations and yet effectively describes the important aspects of the physical system. Several such approximation will be discussed in the following sections.

\subsection{Born-Oppenheimer Approximation}

To overcome the dimensional bottleneck in the simulation of large systems, a set of approximations are proposed which captures the essential physics of the system. One such approximation was proposed by Max Born and J. Robert Oppenheimer in 1927 [1]. This approximation treats the dynamics of the nuclei and the electrons on a different level i.e. the motion of the electrons and the nuclei are separated. The separation comes from the large difference between the nucleus and the electron mass, which leads to the separation of the energy scales of the two entities in the physical system.

The construction starts with an exact ansatz for the many-particle wave-function in Eq. 2.4. The wavefunction is separated into a product of electronic and nuclear wavefunction. The electronic wave function is denoted by $\psi(\vec{x} ; \vec{R})$ with the position of electrons as its arguments. The position vector $\vec{R}=\left\{\vec{R}_{1}, \vec{R}_{2}, \ldots, \vec{R}_{M}\right\}$ of the nuclei, enter as a parameter. The wavefunction of the nuclei is denoted by $\phi(\vec{R}, t)$ with the position of nuclei and time as its argument.

The many-particle wave-function in Eq. 2.4 can be written as [1, 59],

$$
\Phi(\vec{x}, \vec{R}, t)=\sum_{n} \psi_{n}(\vec{x} ; \vec{R}) \phi_{n}(\vec{R}, t)
$$

The full Hamiltonian of the solid-state system in Eq. 2.1 can also be decomposed 
into two parts as,

$$
\hat{\mathcal{H}}=\hat{T}_{n}+\hat{\mathcal{H}}^{\mathrm{BO}}(\vec{R})
$$

where $\hat{\mathcal{H}}^{\mathrm{BO}}$ is referred to as the Born-Oppenheimer Hamiltonian, which only excludes the kinetic energy of the nuclei. The Born-Oppenheimer Hamiltonian is used to study the spectrum of the electrons. The position of the nuclei $\vec{R}_{i}$ in $\hat{\mathcal{H}}^{\mathrm{BO}}$ is considered as a parameter and the Hamiltonian only acts on the Hilbert space of the electrons. The Schrödinger equation for the electronic degrees of freedom is given by

$$
\left[\hat{\mathcal{H}}^{\mathrm{BO}}(\vec{R})-E_{n}^{\mathrm{BO}}(\vec{R})\right] \psi_{n}(\vec{x} ; \vec{R})=0
$$

As evident from Eq. 2.7. the spectrum or the eigen-energy $E_{n}^{\mathrm{BO}}(\vec{R})$ of the electrons, depend parametrically on the position vectors $\vec{R}$ of nuclei. $E_{n}^{\mathrm{BO}}(\vec{R})$ is also termed as the Born-Oppenheimer surface which plays a crucial role in the dynamics of the nuclei. The electronic eigenstates $\psi_{n}(\vec{x} ; \vec{R})$ form an orthonormal basis set, i.e.,

$$
\sum_{\vec{\sigma}} \int d^{3 N} \vec{r} \psi_{n}^{*}(\vec{r}, \vec{\sigma} ; \vec{R}) \psi_{m}(\vec{r}, \vec{\sigma} ; \vec{R})=\delta_{m n}
$$

where $\vec{r}=\left\{\vec{r}_{1}, \vec{r}_{2}, \ldots \vec{r}_{N}\right\}$ and $\vec{\sigma}=\left\{\sigma_{1}, \sigma_{2}, \ldots \sigma_{N}\right\}$ are the set of position vectors and the spins of the N-electrons.

On the other hand the Schrödinger equation for the nuclei wave-functions $\phi_{n}(\vec{R}, t)$ has the following form [[59] and See Chapter 2 of [60]]

$$
\begin{aligned}
i \partial_{t} \phi_{n}(\vec{R}, t) & =\left[\sum_{i=1}^{M}-\frac{\hbar^{2}}{2 M_{i}} \vec{\nabla}_{i}^{2}+E_{n}^{\mathrm{BO}}\right] \phi_{n}(\vec{R}, t) \\
& +\sum_{m}\left[\sum_{i=1}^{M} A_{n m}^{i}(\vec{R}) \vec{\nabla}_{i}+B_{n m}^{i}(\vec{R})\right] \phi_{m}(\vec{R}, t)
\end{aligned}
$$

where the quantities $A_{n m}^{i}(\vec{R})$ and $B_{n m}^{i}(\vec{R})$ are termed as the first and second derivative couplings respectively. The derivative couplings has the form [See Chapter 2 of [60]],

$$
\begin{gathered}
A_{n m}^{i}(\vec{R})=-\frac{\hbar^{2}}{M_{i}}\left\langle\psi_{n}\left|\vec{\nabla}_{i}\right| \psi_{m}\right\rangle \\
B_{n m}^{i}(\vec{R})=-\frac{\hbar^{2}}{2 M_{i}}\left\langle\psi_{n}\left|\vec{\nabla}_{i}^{2}\right| \psi_{m}\right\rangle
\end{gathered}
$$

where the notation of \langle\rangle denotes the integration over electronic degrees of freedom. The derivative couplings depend parametrically on the nuclear position vectors because electronic positions $\vec{r}$ and spin $\vec{\sigma}$ have been explicitly integrated over. 


\subsection{Solid state system in second quantization}

The electronic and the nuclear degrees of freedoms have been separated through the Born-Oppenheimer approximation. As stated earlier, the Born-Oppenheimer surface is the electronic eigenstate of the Born-Oppenheimer Hamiltonian given by,

$$
\hat{\mathcal{H}}^{\mathrm{BO}}=\hat{T}_{e}+\hat{W}_{e-e}+\sum_{i} \hat{v}_{e x t}^{i}
$$

where $\hat{v}_{\text {ext. }}^{i}$ contains the information about the electrostatic potential due to the nuclei and any other external potential acting on the $i$-th electron.

It is understood that the Born-Oppenheimer Hamiltonian is for the fixed nuclear positions $\{\vec{R}\}$. In this section we will express the Hamiltonian in second quantized notation in the Fock space i.e. to extend the Hilbert space and allow the states with different particle numbers. In this space different particle number states are build by successively creating particles in the vacuum.

\subsubsection{Local spin orbitals}

To this direction we introduce single particle states called the local spin orbitals $\chi_{\alpha}(\vec{x})$, which is a two component spinor defined as,

$$
\chi_{\alpha}(\vec{x})=\left\langle\vec{r}, \sigma \mid \chi_{\alpha}\right\rangle:=\left(\begin{array}{c}
\chi_{\alpha}(\vec{r}, \uparrow) \\
\chi_{\alpha}(\vec{r}, \downarrow)
\end{array}\right)
$$

This wave-function has information about the contribution along the spin-up and spin-down direction given by $\chi_{\alpha}(\vec{r} \uparrow)$ and $\chi_{\alpha}(\vec{r}, \downarrow)$ respectively. The amplitude of the wave-function $\left|\chi_{\alpha}^{*}(\vec{x}) \chi_{\alpha}(\vec{x})\right|$ gives the probability of finding a particle at position $\vec{r}$ with spin $\sigma$.

This is a very general notation of the orbitals where spin is treated as a quantum number and it allows to go beyond the spin eigenstates along a particular direction. A local spin orbital, which say is an eigenstate of $\hat{S}_{z}$, has only one non-zero component of the spinor. The advantage of using spin orbital is the fact that it allows to describe orbitals that do not point along the z-direction.

The spin-direction or the magnetization $\vec{m}_{\alpha}(\vec{r})$ at position $\vec{r}$ of the local spin orbitals $\chi_{\alpha}(\vec{x})$, has the form,

$$
\vec{m}_{\alpha}(\vec{r}):=\frac{e \hbar}{2 m_{e}}\left\langle\chi_{\alpha}|\hat{\vec{\sigma}}| \chi_{\alpha}\right\rangle
$$

where $e$ and $m_{e}$ are the charge and the mass of the electron respectively. The vector 
$\hat{\vec{\sigma}}=\left\{\hat{\sigma}_{x}, \hat{\sigma}_{y}, \hat{\sigma}_{z}\right\}$ is a three component vector of Pauli matrices given by

$$
\sigma_{x}=\left(\begin{array}{cc}
0 & 1 \\
1 & 0
\end{array}\right), \quad \sigma_{y}=\left(\begin{array}{cc}
0 & -i \\
i & 0
\end{array}\right), \quad \sigma_{z}=\left(\begin{array}{cc}
1 & 0 \\
0 & -1
\end{array}\right),
$$

\subsubsection{Projector states}

In the absence of orthonormal basis set, one constructs a projector state $\left|\pi_{\alpha}\right\rangle$ for each local orbital $\left|\chi_{\alpha}\right\rangle$ such that it obeys the bi-orthogonality condition,

$$
\left\langle\pi_{\alpha} \mid \chi_{\beta}\right\rangle=\delta_{\alpha \beta}
$$

An arbitrary single particle wave-function $|\psi\rangle=\sum_{\alpha} c_{\alpha}\left|\chi_{\alpha}\right\rangle$ can be decomposed into its contribution of the local orbitals as,

$$
|\psi\rangle=\sum_{\alpha}\left|\chi_{\alpha}\right\rangle\left\langle\pi_{\alpha} \mid \psi\right\rangle
$$

The projection of a general wave-function $|\psi\rangle$ on to a set of local orbitals $\left|\chi_{\alpha}\right\rangle$ will be complete, if,

$$
\sum_{\alpha}\left|\chi_{\alpha}\right\rangle\left\langle\pi_{\alpha}\right|=1
$$

The projector states are introduced to compensate for the in availability of the complete orthonormal basis set of the local spin orbitals. The projector states are identical to the local spin orbitals only if the local spin orbitals form a complete orthonormal basis set.

\subsubsection{Hamiltonian in second quantization}

With the recipe in hand, we introduce the field operators $\hat{\Psi}^{\dagger}(\vec{x})$ and $\hat{\Psi}(\vec{x})$ which creates and annihilates an electron with spin $\sigma$ at position $\vec{r}$ respectively, and rewrite the Born-Oppenheimer Hamiltonian $\hat{\mathcal{H}}^{\mathrm{BO}}$ as,

$$
\begin{aligned}
\hat{\mathcal{H}}^{\mathrm{BO}} & =\int d \vec{x} \hat{\Psi}^{\dagger}(\vec{x})\left[-\frac{\hbar^{2}}{2 m_{e}} \nabla^{2}+v_{\text {ext. }}(\vec{x})\right] \hat{\Psi}(\vec{x}) \\
& +\frac{1}{2} \int d \vec{x} \int d \overrightarrow{x^{\prime}} \hat{\Psi}^{\dagger}(\vec{x}) \hat{\Psi}^{\dagger}\left(\overrightarrow{x^{\prime}}\right) \frac{e^{2}}{4 \pi \epsilon_{0}\left|\vec{r}-\overrightarrow{r^{\prime}}\right|^{2}} \hat{\Psi}\left(\overrightarrow{x^{\prime}}\right) \hat{\Psi}(\vec{x})
\end{aligned}
$$

where $\vec{x}=(\vec{r}, \sigma)$ is as usual the composite index for the position and the spin variable. We use the shorthand $\int d \vec{x}=\sum_{\sigma} \int d \vec{r}$ for the integration over positions and the sum over spin indices. The field operators obey the usual anticommutator relations $\left\{\hat{\Psi}^{\dagger}(\vec{x}), \hat{\Psi}\left(\overrightarrow{x^{\prime}}\right)\right\}=\delta\left(\vec{r}-\overrightarrow{r^{\prime}}\right) \delta_{\sigma, \sigma^{\prime}}$

The creation and annihilation operators in terms of local spin orbitals can be 
expressed through field operators as,

$$
\begin{aligned}
& \hat{c}_{\alpha}^{\dagger}=\sum_{\sigma} \int d \vec{r} \hat{\Psi}^{\dagger}(\vec{r}, \sigma) \pi_{\alpha}(\vec{r}, \sigma) \\
& \hat{c}_{\alpha}=\sum_{\sigma} \int d \vec{r} \pi_{\alpha}^{*}(\vec{r}, \sigma) \hat{\Psi}(\vec{r}, \sigma)
\end{aligned}
$$

where the back transform for the field operators is given by

$$
\begin{aligned}
& \hat{\Psi}^{\dagger}(\vec{r}, \sigma)=\sum_{\alpha} \chi_{\alpha}^{*}(\vec{r}, \sigma) \hat{c}_{\alpha}^{\dagger} \\
& \hat{\Psi}(\vec{r}, \sigma)=\sum_{\alpha} \chi_{\alpha}(\vec{r}, \sigma) \hat{c}_{\alpha}
\end{aligned}
$$

The creation and annihilation operators obey the following anti-commutation rules,

$$
\left\{\hat{c}_{\alpha}^{\dagger}, \hat{c}_{\beta}\right\}=\left\langle\pi_{\alpha} \mid \pi_{\beta}\right\rangle ; \quad\left\{\hat{c}_{\alpha}^{\dagger}, \hat{c}_{\beta}^{\dagger}\right\}=0 ; \quad\left\{\hat{c}_{\alpha}, \hat{c}_{\beta}\right\}=0
$$

The anti-commutation between the creation and annihilation operator is not a delta function, but the overlap between two projector states, and this is due to the fact that the set of local spin orbitals may not necessarily be a complete orthonormal basis set.

Substituting the field operators as defined in Eq. 2.20) into the expression of Born-Oppenheimer Hamiltonian $\hat{\mathcal{H}}^{\mathrm{BO}}$ in Eq.2.18, we get,

$$
\hat{\mathcal{H}}^{\mathrm{BO}}=\sum_{\alpha \beta} h_{\alpha \beta} \hat{c}_{\alpha}^{\dagger} \hat{c}_{\beta}+\frac{1}{2} \sum_{\alpha \beta \gamma \delta} W_{\alpha \beta \gamma \delta} \hat{c}_{\alpha}^{\dagger} \hat{c}_{\beta}^{\dagger} \hat{c}_{\delta} \hat{c}_{\gamma}
$$

with the one-particle hamiltonian $\hat{\boldsymbol{h}}$

$$
h_{\alpha, \beta}=\int d \vec{x} \chi_{a}^{*}(\vec{x})\left(-\frac{\hbar^{2}}{2 m_{e}} \vec{\nabla}^{2}+v_{\text {ext }}(\vec{x})\right) \chi_{\beta}(\vec{x})
$$

The off-diagonal elements of $\boldsymbol{h}$ are named hopping parameters, and the diagonal elements are named orbital energies.

The interaction matrix elements are

$$
W_{\alpha \beta \gamma \delta}=\int d \vec{x} \int d \overrightarrow{x^{\prime}} \frac{\chi_{\alpha}^{*}(\vec{x}) \chi_{\beta}^{*}\left(\overrightarrow{x^{\prime}}\right) \chi_{\gamma}(\vec{x}) \chi_{\delta}\left(\overrightarrow{x^{\prime}}\right)}{4 \pi \epsilon_{0}\left|\vec{r}-\overrightarrow{r^{\prime}}\right|}
$$

The interaction tensor $\hat{\boldsymbol{W}}$ is symmetric under the exchange of extreme indices i.e.,

$$
W_{\alpha \beta, \gamma \delta}=W_{\beta \alpha, \delta \gamma}
$$


which is simply obtained by interchanging the position and spin indices which are integrated over in Eq.(2.24).

In the subsequent discussion, the nuclear positions are assumed to be fixed and therefore for notational simplicity we will give up superscript BO for Born Oppenheimer in Eq. (2.22) and instead use $\hat{\mathcal{H}}$ for the electronic Hamiltonian and $E_{n}$ for the Born-Oppenheimer surface. The value of reduced Planck constant has been set to $\hbar=1$.

\subsection{Grand-canonical potential}

At finite temperature, instead of the ground state, the grand canonical potential is an appropriate quantity. The grand canonical potential $\Omega_{\beta}^{\hat{h}+\hat{W}}$ for the many-body system described by the Hamiltonian $\mathcal{H}$ in Eq. (2.22), is derived from the partition function $\mathcal{Z}$ as,

$$
\begin{aligned}
\Omega_{\beta}^{\hat{\boldsymbol{h}}+\hat{\boldsymbol{W}}} & =-\frac{1}{\beta} \ln \mathcal{Z} \\
\mathcal{Z} & =\operatorname{Tr}\left(e^{-\beta(\hat{\boldsymbol{h}}+\hat{\boldsymbol{W}}-\mu \hat{\mathcal{N}})}\right)
\end{aligned}
$$

where $\beta=1 / k_{B} T$ is the inverse temperature and $\hat{\mathcal{N}}$ is the number operator which has the form

$$
\hat{\mathcal{N}}=\sum_{\alpha \beta}\left\langle\chi_{\alpha} \mid \chi_{\beta}\right\rangle \hat{c}_{\alpha}^{\dagger} \hat{c}_{\beta}
$$

The trace in Eq.(2.26) is over any complete set of basis states of the full-Hamiltonian. This tracing suffers from the exponential bottleneck and thus exact evaluation of the grand potential for a many body system is hard.

The grand potential is an important quantity in thermodynamics. All the thermodynamic properties of a system can be derived from the grand potential, through partial derivatives with respect to thermodynamic variables, for eg.

$$
\begin{aligned}
\langle N\rangle & =-\frac{\partial \Omega_{\beta}^{\hat{\boldsymbol{h}}+\hat{\boldsymbol{W}}}}{\partial \mu} \\
S & =-\frac{\partial \Omega_{\beta}^{\hat{\boldsymbol{h}}+\hat{\boldsymbol{W}}}}{\partial T}
\end{aligned}
$$

where $\langle N\rangle$ is average number of particle and $S$ is the entropy of the system.

Since the exact evaluation of grand-canonical potential for a many-body system is extremely hard, one proceeds towards development of approximate schemes which can describe a many-body system effectively. To this direction, important 
formalisms like Density-Functional Theory (DFT), reduced Density Matrix Functional Theory (rDMFT), many-body approaches like perturbation theory and the Dynamical Mean Field Theory (DMFT) etc, have been proposed which provides an approximate yet effective approach to study complicated many-body systems.

In the above mentioned formalisms, static objects like density, one-particle reduced density matrices, two-particle density matrices, and dynamical objects like Green's function and the self-energy, are used quite often. These objects are mostly used to evaluate ground state energy or the grand-canonical potential, spectral functions etc. In the following section, we briefly discuss some of these important objects and their relation to each other.

\subsection{Important quantities}

\subsubsection{Density matrices}

The density matrices [61] are objects which encodes the same information as the many particle wave functions. The full many particle density matrix $\hat{\Gamma}$ for a statistical ensemble $\left\{P_{i}, \Psi_{i}(\{\vec{x}\})\right\}$ has the form,

$$
\Gamma\left(\vec{x}_{1}, \vec{x}_{2} \ldots \vec{x}_{N} ;{\overrightarrow{x^{\prime}}}_{1},{\overrightarrow{x^{\prime}}}_{2} \ldots{\overrightarrow{x^{\prime}}}_{N}\right)=\sum_{i} P_{i} \Psi_{i}\left(\vec{x}, \vec{x}_{2} \ldots \vec{x}_{N}\right) \Psi_{i}^{*}\left({\overrightarrow{x^{\prime}}}_{1},{\overrightarrow{x^{\prime}}}_{2} \ldots{\overrightarrow{x^{\prime}}}_{N}\right)
$$

$\hat{\Gamma}$ is a positive semi-definite hermitian matrix with trace equal to one.

The statistical average of any operator $\hat{A}$ for the ensemble $\left\{P_{i}, \Psi_{i}(\{\vec{x}\})\right\}$ can be evaluated from the many-particle density matrix $\hat{\Gamma}$ as,

$$
\langle\hat{A}\rangle=\operatorname{Tr}(\hat{\Gamma} \hat{A})
$$

In solid-state physics, the typical operators one deal with are the one-body objects like kinetic energy, magnetization etc. and the two-body operators like $\hat{W}$, the magnetic susceptibility etc. In the evaluation of the averages of such operators, the full knowledge of many-particle density matrix is redundant and to that effect we introduce the concept of one and two-particle reduced density matrices.

\section{One-particle reduced density matrix}

The one-particle reduced density matrix is denoted by an operator $\hat{\rho}$ and is an essential entity for the evaluation of the expectation value of one-body operators such as kinetic energy, magnetization etc. of the electronic system.

The one-particle reduced density matrix is obtained from an ensemble $\left\{P_{i}, \Psi_{i}(\{\vec{x}\})\right\}$ of an N-electron system, by systematically tracing out the positions of $N-1$ electrons 
as,

$$
\rho\left(\vec{x}, \overrightarrow{x^{\prime}}\right):=\sum_{i} P_{i}\left(N \int \Psi_{i}\left(\vec{x}, \vec{x}_{2} \ldots \vec{x}_{N}\right) \Psi_{i}^{*}\left(\vec{x}^{\prime}, \vec{x}_{2} \ldots \vec{x}_{N}\right) d \vec{x}_{2} \ldots d \vec{x}_{N}\right)
$$

It is important to note that one-particle reduced density matrix has two positions in its argument. However, these are the positions of only one electron. The diagonal part of the one-particle density matrix gives the spatial particle density denoted by,

$$
n^{(1)}(\vec{r})=\sum_{\sigma} \rho(\vec{x}, \vec{x})
$$

The one-particle reduced density matrix can also be represented in terms of field operators as,

$$
\rho\left(\vec{x}, \overrightarrow{x^{\prime}}\right):=\sum_{i} P_{i}\left\langle\Psi_{i}\left|\hat{\Psi}^{\dagger}\left(\overrightarrow{x^{\prime}}\right) \hat{\Psi}(\vec{x})\right| \Psi_{j}\right\rangle
$$

which, when expressed in terms of creation and annihilation operators, has the form,

$$
\rho\left(\vec{x}, \overrightarrow{x^{\prime}}\right)=\sum_{\alpha \beta} \chi_{\alpha}(\vec{x})\left(\sum_{i} P_{i}\left\langle\Psi_{i}\left|\hat{c}_{\beta}^{\dagger} \hat{c}_{\alpha}\right| \Psi_{i}\right\rangle\right) \chi_{\beta}^{*}\left(\overrightarrow{x^{\prime}}\right)
$$

where we define the matrix elements of one-particle reduced density matrix in local orbital basis as,

$$
\rho_{\alpha \beta}=\sum_{i} P_{i}\left\langle\Psi_{i}\left|\hat{c}_{\beta}^{\dagger} \hat{c}_{\alpha}\right| \Psi_{i}\right\rangle
$$

The eigen-decomposition of one particle reduced density matrix $\rho\left(\vec{x}, \overrightarrow{x^{\prime}}\right)$ has the form,

$$
\rho\left(\vec{x}, \overrightarrow{x^{\prime}}\right)=\sum_{n} f_{n} \phi_{n}(\vec{x}) \phi_{n}^{*}\left(\vec{x}^{\prime}\right)
$$

where the eigenvalues $f_{n}$ are called the occupations and the eigen vectors $\phi_{n}(\vec{x})$ are also termed as the natural orbitals.

The one-particle reduced density matrix obtained from an anti-symmetrized Nparticle wave function is an N-representable matrix [62, 63, 64] which means that eigenvalues follow the conditions,

$$
\sum_{n} f_{n}=N, \quad \forall n, 0 \leq f_{n} \leq 1
$$

The statement is more general which states that any matrix that can be represented by an ensemble of fermionic N-particle wave functions is an N-representable matrix.

The one-particle reduced density matrix comes in handy in the evaluation of ensemble average of one-body terms in the Hamiltonian in Eq.(2.18). Using the definition of one-particle reduced density matrix in Eq. (2.33), it can be shown that 
the ensemble average has the form,

$$
\int d \vec{x} \lim _{\vec{x}^{\prime} \rightarrow \vec{x}}\left(-\frac{\nabla_{\vec{r}}^{2}}{2}+V_{\text {ext. }}\right) \rho\left(\vec{x}, \vec{x}^{\prime}\right)
$$

\section{Two particle density matrix}

The second important quantity for systems with two-particle interactions is the two-particle density matrix denoted by $\rho^{(2)}\left(\vec{x}_{1}, \vec{x}_{2} ; \vec{x}_{1}^{\prime}, \vec{x}_{2}^{\prime}\right)$. The two particle reduced density matrix is obtained from $\mathrm{N}$-particle wave functions by integrating out the remaining $N-2$ degrees of freedom as,

$$
\rho^{(2)}\left(\vec{x}_{1}, \vec{x}_{2} ; \vec{x}_{1}{ }_{1}, \vec{x}^{\prime}\right)=N(N-1) \int \Psi\left(\vec{x}_{1}, \vec{x}_{2}, \vec{x}_{3} \ldots \vec{x}_{N}\right) \Psi^{*}\left(\vec{x}^{\prime}, \vec{x}_{2}, \vec{x}_{3} \ldots \vec{x}_{N}\right) d \vec{x}_{3} \ldots d \vec{x}_{N}
$$

The two particle density matrix has four arguments, two for each electron. The two particle density operator is used to evaluate the expectation value of two-body operators (like Coulomb interaction) in Eq. (2.18), and has the form,

$$
\frac{1}{2} \int d \vec{x} \int d \overrightarrow{x^{\prime}} \frac{e^{2}}{4 \pi \epsilon_{0}\left|\vec{r}-\vec{r}^{\prime}\right|^{2}} \rho^{(2)}\left(\vec{x}, \overrightarrow{x^{\prime}} ; \vec{x}, \overrightarrow{x^{\prime}}\right)
$$

\section{Hole-function}

The physical quantity which is more identifiable is the two-particle density $n^{(2)}\left(\vec{r}, \vec{r}^{\prime}\right)$ given by,

$$
n^{(2)}\left(\vec{r}, \overrightarrow{r^{\prime}}\right)=\sum_{\sigma, \sigma^{\prime}} \rho^{(2)}\left(\vec{x}, \overrightarrow{x^{\prime}} ; \vec{x}, \overrightarrow{x^{\prime}}\right)
$$

i.e. the spin-averaged diagonal elements of two particle reduced density matrix gives the two particle density. From the physical perspective, for an uncorrelated system, the two particle density is the product of densities at two positions

$$
n^{(2)}\left(\vec{r}, \overrightarrow{r^{\prime}}\right)=n^{(1)}(\vec{r}) n^{(1)}\left(\overrightarrow{r^{\prime}}\right)
$$

The conditional density $\frac{n^{(2)}\left(\vec{r}, \overrightarrow{r^{\prime}}\right)}{n^{(1)}(\vec{r})}$ is the density at $\overrightarrow{r^{\prime}}$ as seen by the electron at $\vec{r}$ which experiences density due to $N-1$ electrons. This conditional density is given in terms of a quantity called hole function $h\left(\vec{r}, \vec{r}^{\prime}\right)$ as,

$$
\frac{n^{(2)}\left(\vec{r}, \overrightarrow{r^{\prime}}\right)}{n^{(1)}(\vec{r})}=n^{(1)}\left(\vec{r}^{\prime}\right)+h\left(\vec{r}, \overrightarrow{r^{\prime}}\right)
$$

The hole function has the following properties,

- Since the electron at any position sees density due to other $N-1$ electrons, 
the hole function should always integrate to -1 ,

$$
\int d \overrightarrow{r^{\prime}} h\left(\vec{r}, \overrightarrow{r^{\prime}}\right)=-1
$$

- Electrons obey Pauli principle i.e. two electrons of same spin cannot be at the same position, which demands

$$
n^{(2)}(\vec{r}, \vec{r})=0
$$

- At large distances $\left|\vec{r}-\overrightarrow{r^{\prime}}\right| \rightarrow \infty$, the two particle density is simply the product of individual densities, and thus the hole function should satisfy,

$$
h\left(\vec{r}, \overrightarrow{r^{\prime}}\right) \rightarrow 0 \quad \text { for } \quad\left|\vec{r}-\overrightarrow{r^{\prime}}\right| \rightarrow \infty
$$

A detailed discussion on the properties of the hole function can be found in the lecture notes of P. Blöchl [See Chapter 1 of [65]]

\subsubsection{Green's function and self energy}

In this subsection we will discuss important dynamical objects which are used extensively in many-body approaches to investigate solid-state systems.

\section{Green's function}

At finite temperature, the single particle Matsubara Green's function [66] for the many body Hamiltonian $\hat{\mathcal{H}}=\hat{\boldsymbol{h}}+\hat{\boldsymbol{W}}$ has the form,

$$
\begin{aligned}
\mathcal{G}_{\alpha \beta}\left(\tau, \tau^{\prime}\right) & :=-\left\langle\mathcal{T} \hat{c}_{\alpha}(\tau) \hat{c}_{\beta}^{\dagger}\left(\tau^{\prime}\right)\right\rangle \\
& =-\frac{1}{\mathcal{Z}} \operatorname{Tr}\left\{e^{-\beta(\hat{\boldsymbol{h}}+\hat{\boldsymbol{W}}-\mu \hat{\mathcal{N}})} \mathcal{T}\left(\hat{c}_{\alpha}(\tau) \hat{c}_{\beta}^{\dagger}\left(\tau^{\prime}\right)\right)\right\} \\
\mathcal{Z} & =\operatorname{Tr}\left\{e^{-\beta(\hat{\boldsymbol{h}}+\hat{\boldsymbol{W}}-\mu \hat{\mathcal{N}})}\right\}
\end{aligned}
$$

where $\tau$ is the imaginary time, obtained from the real time $t$ through the Wick's rotation $\tau=i t$. The time-ordering operator $\mathcal{T}$ orders the operator in decreasing order of time i.e. the operators with smaller time are moved towards right.

The time-dependence of the operators are obtained as,

$$
\begin{aligned}
& \hat{c}_{\alpha}(\tau)=e^{(\hat{\mathcal{H}}-\mu \hat{\mathcal{N}}) \tau} \hat{c}_{\alpha} e^{-(\hat{\mathcal{H}}-\mu \hat{\mathcal{N}}) \tau} \\
& \hat{c}_{\beta}^{\dagger}(\tau)=e^{(\hat{\mathcal{H}}-\mu \hat{\mathcal{N}}) \tau} \hat{c}_{\beta}^{\dagger} e^{-(\hat{\mathcal{H}}-\mu \hat{\mathcal{N}}) \tau}
\end{aligned}
$$

Using the fact that the total Hamiltonian $\hat{\mathcal{H}}$ is time-independent and the operators 
can be permuted cyclically within the trace, it can be shown that the Green's function $\mathcal{G}_{\alpha \beta}\left(\tau, \tau^{\prime}\right)$ depends only on the time difference $\tau-\tau^{\prime}$ in Eq. 2.47). Therefore it is convenient to set $\tau^{\prime}=0$.

It can also be shown easily that the Green's function $\mathcal{G}_{\alpha \beta}(\tau)$ follows an important property,

$$
\mathcal{G}_{\alpha \beta}(\tau+\beta)=\eta \mathcal{G}_{\alpha \beta}(\tau)
$$

where $\eta= \pm 1$ for bosons and fermions respectively. This property helps in restricting the imaginary time domain to a finite set $\tau \in[0, \beta]$.

Due to this periodicity and anti-periodicty of bosonic and fermionic Green's function respectively, we can do the Fourier transformation of $\mathcal{G}(\tau)$ and the back transformation as,

$$
\begin{aligned}
\mathcal{G}\left(i \omega_{n}\right) & =\frac{1}{2} \int_{-\beta}^{\beta} d \tau e^{i \omega_{n} \tau} \mathcal{G}(\tau) \\
\mathcal{G}(\tau) & =\frac{1}{\beta} \sum_{\omega_{n}} e^{-i \omega_{n} \tau} \mathcal{G}\left(i \omega_{n}\right) \\
\omega_{n} & = \begin{cases}\frac{(2 n+1) \pi}{\beta} & \text { for fermions } \\
\frac{2 n \pi}{\beta} & \text { for bosons }\end{cases}
\end{aligned}
$$

\section{Self-energy}

Another important quantity which is used quite often in the many-body approaches for the strongly correlated electron system is the self-energy. The self energy relates a two- particle propagator to one-particle Green's function. The two-particle propagator arises in the expression for the equation of motion of one particle Green's function. The equation of motion for one-particle Green's function $\mathcal{G}_{\alpha \beta}(\tau)$ has the form,

$$
\begin{aligned}
\frac{\partial}{\partial \tau} \mathcal{G}_{\alpha \beta}(\tau) & =\partial_{\tau}\left[-\Theta(\tau)\left\langle\hat{c}_{\alpha}(\tau) \hat{c}_{\beta}^{\dagger}(0)\right\rangle+\Theta(-\tau)\left\langle\hat{c}_{\beta}^{\dagger}(0) \hat{c}_{\alpha}(\tau)\right\rangle\right] \\
& =\delta(\tau)\left[-\left\langle\hat{c}_{\alpha}(\tau) \hat{c}_{\beta}^{\dagger}(0)\right\rangle-\left\langle\hat{c}_{\beta}^{\dagger}(0) \hat{c}_{\alpha}(\tau)\right\rangle\right] \\
& -\Theta(\tau)\left\langle\partial_{\tau} \hat{c}_{\alpha}(\tau) \hat{c}_{\beta}^{\dagger}(0)\right\rangle+\Theta(-\tau)\left\langle\hat{c}_{\beta}^{\dagger}(0) \partial_{\tau} \hat{c}_{\alpha}(\tau)\right\rangle
\end{aligned}
$$

The time derivative of annihilation operator $\hat{c}_{\alpha}(\tau)$ is evaluated from the Heisenberg equation of motion as,

$$
\partial_{\tau} \hat{c}_{\alpha}(\tau)=\left[\hat{\mathcal{H}}-\mu \hat{\mathcal{N}}, \hat{c}_{\alpha}(\tau)\right]
$$

The commutator [,] in Eq.2.52 can be evaluated, given the form of Hamiltonian in Eq. 2.22 as,

$$
\left[\hat{\mathcal{H}}-\mu \hat{\mathcal{N}}, \hat{c}_{\alpha}(\tau)\right]=\sum_{\gamma}\left(h_{\alpha \gamma}-\mu \delta_{\alpha \gamma}\right) \hat{c}_{\gamma}(\tau)+\sum_{\gamma, \delta, \lambda} W_{\alpha \gamma, \delta \lambda} \hat{c}_{\gamma}^{\dagger}(\tau) \hat{c}_{\lambda}(\tau) \hat{c}_{\delta}(\tau)
$$


The equation of motion for the one particle Green's function in Eq.(2.51), can be expanded using Eq.2.53 as,

$$
\begin{aligned}
\frac{\partial}{\partial \tau} \mathcal{G}_{\alpha \beta}(\tau) & =-\delta(\tau) \delta_{\alpha \beta}-\sum_{\gamma}\left(h_{\alpha \gamma}-\mu \delta_{\alpha \gamma}\right) \mathcal{G}_{\gamma \beta}(\tau) \\
& +\sum_{\gamma, \delta, \lambda} W_{\alpha \gamma, \delta \lambda}\left\langle\mathcal{T} \hat{c}_{\gamma}^{\dagger}(\tau) \hat{c}_{\lambda}(\tau) \hat{c}_{\delta}(\tau) \hat{c}_{\beta}^{\dagger}(0)\right\rangle
\end{aligned}
$$

As evident from Eq.2.54), the equation of motion for a one particle Green's function requires the knowledge of two particle correlator arisng due to the presence of two body operator $\hat{W}$. The two body correlator is related to one-particle Green's function through self-energy $\Sigma(\tau)$ as,

$$
\sum_{\eta} \int_{0}^{\beta} d \tau^{\prime} \Sigma_{\alpha \eta}\left(\tau-\tau^{\prime}\right) \mathcal{G}_{\eta \beta}\left(\tau^{\prime}\right)=-\sum_{\gamma \delta \lambda} W_{\alpha \gamma, \delta \lambda}\left\langle\mathcal{T} \hat{c}_{\gamma}^{\dagger}(\tau) \hat{c}_{\lambda}(\tau) \hat{c}_{\delta}(\tau) \hat{c}_{\beta}^{\dagger}(0)\right\rangle
$$

The equation of motion for the one particle Green's function in Eq. 2.54) attains the form,

$$
\begin{aligned}
\frac{\partial}{\partial \tau} \mathcal{G}_{\alpha \beta}(\tau) & =-\delta(\tau) \delta_{\alpha \beta}-\sum_{\gamma}\left(h_{\alpha \gamma}-\mu \delta_{\alpha \gamma}\right) \mathcal{G}_{\gamma \beta}(\tau) \\
& -\sum_{\eta} \int_{0}^{\beta} d \tau^{\prime} \Sigma_{\alpha \eta}\left(\tau-\tau^{\prime}\right) \mathcal{G}_{\eta \beta}\left(\tau^{\prime}\right)
\end{aligned}
$$

Using the fourier transformation for the Green's function and the self energy the equation of motion in Eq.(2.56) can be casted in the well known Dyson equation which has the form,

$$
\mathcal{G}_{\alpha \beta}\left(i \omega_{n}\right)=\left[i \omega_{n} \mathbf{1}+\mu \mathbf{1}-\boldsymbol{h}-\boldsymbol{\Sigma}\left(i \omega_{n}\right)\right]_{\alpha \beta}^{-1}
$$

\subsubsection{Lehmann representation of the Green's function}

In the later discussions on the density matrix functional derived from the Green's function, the search for an approximate Green's function will be a crucial task. For that purpose, we present the most useful representation of the Green's function, namely the Lehmann representation. In this representation the Green's function has the following form, 


$$
\mathcal{G}_{\alpha \beta}\left(i \omega_{n}\right)=\sum_{m n}\left\langle\Phi_{m}\left|\hat{c}_{\alpha}\right| \Phi_{n}\right\rangle\left\langle\Phi_{n}\left|\hat{c}_{\beta}^{\dagger}\right| \Phi_{m}\right\rangle \frac{e^{-\beta\left(E_{m}-\mu N_{m}-\Omega\right)}+e^{-\beta\left(E_{n}-\mu N_{n}-\Omega\right)}}{i \omega_{n}+E_{m}-E_{n}+\mu}
$$

We assume that the full spectrum i.e the eigenvalues $\left\{E_{m}\right\}$ and the eigen-vectors $\left\{\left|\Phi_{m}\right\rangle\right\}$ of the Hamiltonian $\hat{\mathcal{H}}-\mu \mathcal{N}$ are available, and the eigen-vectors are orthonormal and form the complete basis set in the Fock space, i.e.

$$
\begin{aligned}
\sum_{m}\left|\Phi_{m}\right\rangle\left\langle\Phi_{m}\right| & =\mathbf{1} \\
\left\langle\Phi_{m} \mid \Phi_{n}\right\rangle & =\delta_{m n}
\end{aligned}
$$

The Green's function can further be written in a convenient form using the spectral function $\boldsymbol{A}$ as,

$$
\mathcal{G}_{\alpha \beta}\left(i \omega_{n}\right)=\int_{-\infty}^{\infty} d \epsilon \frac{A_{\alpha \beta}(\epsilon)}{i \omega_{n}+\mu-\epsilon}
$$

where

$$
\begin{aligned}
A_{\alpha \beta}(\epsilon) & =\sum_{m n}\left\langle\Phi_{m}\left|\hat{c}_{\alpha}\right| \Phi_{n}\right\rangle\left\langle\Phi_{n}\left|\hat{c}_{\beta}^{\dagger}\right| \Phi_{m}\right\rangle\left(e^{-\beta\left(E_{m}-\mu N_{m}-\Omega\right)}+e^{-\beta\left(E_{n}-\mu N_{n}-\Omega\right)}\right) \delta\left(\epsilon-\left(E_{n}-E_{m}\right)\right) \\
& =\sum_{n} e^{-\beta\left(E_{n}-\mu N_{n}-\Omega\right)}\left\{\sum_{m}\left\langle\Phi_{n}\left|\hat{c}_{\alpha}\right| \Phi_{m}\right\rangle\left\langle\Phi_{m}\left|\hat{c}_{\beta}^{\dagger}\right| \Phi_{n}\right\rangle \delta\left(\epsilon-\left(E_{m}-E_{n}\right)\right)\right. \\
& \left.+\sum_{m}\left\langle\Phi_{n}\left|\hat{c}_{\beta}^{\dagger}\right| \Phi_{m}\right\rangle\left\langle\Phi_{m}\left|\hat{c}_{\alpha}\right| \Phi_{n}\right\rangle \delta\left(\epsilon-\left(E_{n}-E_{m}\right)\right)\right\} \\
& =A_{\alpha \beta}^{\text {elec. }}(\epsilon)+A_{\alpha \beta}^{\text {hole }}(\epsilon)
\end{aligned}
$$

In the last part the spectral function $A_{\alpha \beta}(\epsilon)$ has been divided in two parts, first of which represents the spectral function for the electrons and the second part represents the holes.

\subsubsection{Relation between one particle density matrix and the Green's function}

In this subsection we show the relation between the one particle Green's function $\mathcal{G}(\tau)$ and physical quantities like one-particle reduced density matrix $\boldsymbol{\rho}$. The relation between the Green's function and one-particle reduced density matrix is also important for our future discussion on the density matrix functionals.

The one-particle reduced density matrix $\boldsymbol{\rho}$ for the system given by Hamiltonian 
$\hat{\mathcal{H}}-\mu \mathcal{N}$ is defined as,

$$
\rho_{\alpha \beta}=\operatorname{Tr}\left\{e^{-\beta(\hat{\mathcal{H}}-\mu \mathcal{N}-\Omega)} \hat{c}_{\beta}^{\dagger} \hat{c}_{\alpha}\right\}
$$

If we compare the expression in Eq. (2.62) with the definition of the single particle Green's function in Eq. (2.47), we can conclude that $\boldsymbol{\rho}$ is nothing but the Green's function at imaginary time $\tau \rightarrow 0^{-}$i.e.

$$
\begin{aligned}
\boldsymbol{\rho} & =\mathcal{G}\left(\tau \rightarrow 0^{-}\right) \\
& =\frac{1}{\beta} \sum_{\omega_{n}} e^{i \omega_{n} 0^{+}} \mathcal{G}\left(i \omega_{n}\right)
\end{aligned}
$$

The factor $e^{i \omega_{n} 0^{+}}$is also important for the numerical purpose, since it ensures convergence of the Matsubara frequency sum.

The one particle density matrix can also be obtained from the hole part of the spectral function $A_{\alpha \beta}^{\text {hole }}(\epsilon)$ by integrating over the entire energy range as,

$$
\begin{aligned}
\rho_{\alpha \beta} & =\int_{-\infty}^{\infty} d \epsilon A_{\alpha \beta}^{\text {hole }}(\epsilon) \\
& =\sum_{m n} e^{-\beta\left(E_{n}-\mu N_{n}-\Omega\right)}\left\langle\Phi_{n}\left|\hat{c}_{\beta}^{\dagger}\right| \Phi_{m}\right\rangle\left\langle\Phi_{m}\left|\hat{c}_{\alpha}\right| \Phi_{n}\right\rangle \\
& =\operatorname{Tr}\left\{e^{-\beta(\hat{\mathcal{H}}-\mu \mathcal{N}-\Omega)} \hat{c}_{\beta}^{\dagger} \hat{c}_{\alpha}\right\}
\end{aligned}
$$

\subsubsection{Relation between self energy and the interaction energy}

The additional knowledge of the self energy $\Sigma(\tau)$ also helps in the evaluation of the interaction energy. The average interaction energy is,

$$
\langle\hat{\boldsymbol{W}}\rangle=\frac{1}{2} \sum_{\alpha \beta \gamma \delta} W_{\alpha \beta \gamma \delta}\left\langle\hat{c}_{\alpha}^{\dagger} \hat{c}_{\beta}^{\dagger} \hat{c}_{\delta} \hat{c}_{\gamma}\right\rangle
$$

where $\langle\hat{\boldsymbol{W}}\rangle=\operatorname{Tr}\left\{e^{-\beta(\hat{\mathcal{H}}-\mu \mathcal{N}-\Omega)} \hat{\boldsymbol{W}}\right\}$. The self energy defined in Eq.2.55 can be reformulated in a way such that the time ordering operator can be evaluated,

$$
\sum_{\eta} \int_{0}^{\beta} d \tau^{\prime} \Sigma_{\alpha \eta}\left(0-\tau^{\prime}\right) \mathcal{G}_{\eta \beta}\left(\tau^{\prime}, 0^{+}\right)=\sum_{\gamma \delta \lambda} W_{\alpha \gamma, \delta \lambda}\left\langle\hat{c}_{\beta}^{\dagger}\left(0^{+}\right) \hat{c}_{\gamma}^{\dagger}(0) \hat{c}_{\lambda}(0) \hat{c}_{\delta}(0)\right\rangle
$$


Thus relabelling $\beta=\alpha$ on the both side of Eq.2.66) and summing over $\alpha$ we get,

$$
\begin{aligned}
\langle\hat{\boldsymbol{W}}\rangle & =\frac{1}{2} \sum_{\eta \alpha} \int_{0}^{\beta} d \tau^{\prime} \Sigma_{\alpha \eta}\left(0-\tau^{\prime}\right) \mathcal{G}_{\eta \alpha}\left(\tau^{\prime}, 0^{+}\right) \\
& =\frac{1}{2 \beta} \sum_{\eta \alpha} \sum_{\omega_{n}} e^{i \omega_{n} 0^{+}} \Sigma_{\alpha \eta}\left(i \omega_{n}\right) \mathcal{G}_{\eta \alpha}\left(i \omega_{n}\right) \\
& =\frac{1}{2 \beta} \sum_{\omega_{n}} e^{i \omega_{n} 0^{+}} \operatorname{Tr}\left\{\boldsymbol{\Sigma}\left(i \omega_{n}\right) \mathcal{G}\left(i \omega_{n}\right)\right\}
\end{aligned}
$$

where we have used the Fourier transformation $\Sigma\left(i \omega_{n}\right)$ of the self-energy, defined as,

$$
\Sigma\left(i \omega_{n}\right)=\int_{0}^{\beta} d \tau e^{i \omega_{n} \tau} \Sigma(\tau)
$$

\subsection{Gilbert theorem and the reduced density matrix functional}

Equipped with the knowledge of all the important ingredients, the stage is set to discuss the schemes used to evaluate the grand-canonical potential of Eq. (2.26). To overcome the complexity of the exact evaluation of the grand canonical potential (specifically the ground-state energy at T=0), Hohenberg and Kohn [2] in 1964, provided an exact mapping of the interacting electrons at $T=0$ to a system of noninteracting quasi particles also referred as the Kohn-Sham system [3]. They showed that the ground state property of the interacting system is contained in the ground state density $n_{0}(\vec{r})$. The mapping to an non-interacting Kohn-Sham system assumes that the external potential which is a one body operator is purely local. The locality of the external potential leads to a one-one mapping between the non-degenerate exact ground state wave function and the density.

$$
\Psi_{G S}\left(\vec{x}_{1}, . ., \vec{x}_{N}\right) \leftrightarrow n_{0}(\vec{r})
$$

This one-one mapping ensures the existence of density functionals. The entire complexity of the problem is encoded in the density functional for which several approximations have been tested successfully on different materials.

In 1975 T. Gilbert [6] extended the Hohenberg-Kohn theorem for the non-local external potentials. He proved that for the non-local external potential, there is oneone mapping between the ground state wavefunction and the one particle reduced density matrix,

$$
\Psi_{G S}\left(\vec{x}_{1}, . ., \vec{x}_{N}\right) \leftrightarrow \rho_{0}\left(\vec{x}, \overrightarrow{x^{\prime}}\right)
$$

This one to one mapping ensures that all the ground state expectation values can be written as a functional of the ground state one-particle reduced density matrix. 
This in principle ensures that even the expectation values of two-body operators (See Eq. (2.40) can be written as a functional of one-particle reduced density matrix.

The full complexity in the evaluation of the ensemble average of interaction terms are clubbed in a quantity called reduced density matrix functional $F_{\beta}^{\hat{\boldsymbol{W}}}[\boldsymbol{\rho}]$ and the grand-canonical potential is obtained by minimzation in the space of N-representable one-particle reduced density matrix $\boldsymbol{\rho}$ as,

$$
\Omega_{\beta}^{\hat{\boldsymbol{h}}+\hat{\boldsymbol{W}}}=\min _{\boldsymbol{\rho}}\left[\operatorname{Tr}(\boldsymbol{h} \boldsymbol{\rho})+F_{\beta}^{\hat{\boldsymbol{W}}}[\boldsymbol{\rho}]\right]
$$

The reduced density matrix functional $F_{\beta}^{\hat{W}}[\boldsymbol{\rho}]$ on the other hand can formally be defined as a Legendre transformation of grand-canonical potential as,

$$
F_{\beta}^{\hat{W}}[\boldsymbol{\rho}]=\max _{h_{\alpha \beta}}\left[\Omega_{\beta}^{\hat{h}+\hat{\boldsymbol{W}}}-\operatorname{Tr}(\boldsymbol{h} \boldsymbol{\rho})\right]
$$

It is evident from the definition, that the density matrix functional $F_{\beta}^{\hat{W}}[\boldsymbol{\rho}]$ is universal i.e. it only depends on the interaction $\hat{\boldsymbol{W}}$ and not on the one particle Hamiltonian $\hat{\boldsymbol{h}}$. It also shows that the complexity to evaluate density matrix functional is same as the evaluation of grand canonical potential.

\section{Ways to evaluate density matrix functional}

The practical evaluation of the density matrix functional is however done via three different approaches. In Sec. 2.6.1 and Sec. 2.6.2 we discuss the wave-function and the Green's function based approaches respectively. These two approaches provide an exact expression for the density matrix functional. In Sec. 2.6 .3 we discuss the state of the art parametrized density matrix functionals which are most commonly used in the rDMFT community.

\subsubsection{Wave-function based approach}

The wave-function based approach [67] is the most transparent and straightforward way to define the density matrix functional. In this approach one evaluates the grand potential as a minimum of a functional over an ensemble of fermionic many body wave-functions $\left.\psi_{i}\right\rangle$ in the Fock space. The ensemble of wave-functions are characterized by their respective probabilities $P_{j}$. Thus the optimization is done over an exponentially large space of many body wave-functions and the probabilities as 68],

$$
\begin{aligned}
\Omega_{\beta}^{\hat{\boldsymbol{h}}+\hat{\boldsymbol{W}}=} & \min _{0 \leq P_{j} \leq 1,\left|\psi_{j}\right\rangle} \operatorname{stat}_{\boldsymbol{\Lambda}, \lambda}\left\{\frac{1}{\beta} \sum_{j} P_{j} \ln P_{j}+\sum_{j} P_{j}\left\langle\psi_{j}|\hat{\boldsymbol{h}}+\hat{\boldsymbol{W}}-\mu \hat{\boldsymbol{N}}| \psi_{j}\right\rangle\right. \\
& \left.-\sum_{i j} \Lambda_{i j}\left(\left\langle\psi_{i} \mid \psi_{j}\right\rangle-\delta_{i j}\right)-\lambda \sum_{j}\left(\sum_{j} P_{j}-1\right)\right\}
\end{aligned}
$$


The first part in Eq. 2.73) amounts to the $-T S$ or the entropy part due to finite temperature. The second part is the average of total energy of the system including the particle reservoir. The third and fourth part are due to the fact that during the course of optimization, many particle wavefunctions $\left|\psi_{j}\right\rangle$ and the respective probabilities $P_{j}$ have to follow certain constraints. The orthogonality of the wavefunctions and the constraint that the probabilities should add to unity are enforced using the Lagrange multipliers [69] $\boldsymbol{\Lambda}$ and $\lambda$ respectively. The stationarity with respect to the Lagrange multipliers means either the extrema or the saddle point.

The minimization in Eq. 2.73) is a constrained search problem over many particle wave-functions space. It is performed via two step procedure,

1. In the first step, optimization is done over a subspace of many particle wavefunctions $\left|\psi_{j}\right\rangle$ which produces the one-particle reduced density matrix $\rho$ defined as,

$$
\rho_{\alpha \beta}:=\sum_{j} P_{j}\left\langle\psi_{j}\left|\hat{c}_{\beta}^{\dagger} \hat{c}_{\alpha}\right| \psi_{j}\right\rangle
$$

In this restricted space of many particle wave-functions, the search for the minima is carried out.

2. In the second step, the optimization is done in the space of N-representable one particle reduced density matrices.

The first step in the optimization scheme defines the central quantity of r-DMFT called the reduced density matrix functional, which we denote by $F_{\beta}^{\hat{W}}[\boldsymbol{\rho}]$, and has the form,

$$
\begin{aligned}
F_{\beta}^{\hat{\boldsymbol{W}}}[\boldsymbol{\rho}]= & \min _{0 \leq P_{j} \leq 1,\left|\psi_{j}\right\rangle} \operatorname{stat}_{\boldsymbol{h}^{\prime}, \boldsymbol{\Lambda}, \lambda}\left\{\frac{1}{\beta} \sum_{j} P_{j} \ln P_{j}+\sum_{j} P_{j}\left\langle\psi_{j}|\hat{\boldsymbol{W}}| \psi_{j}\right\rangle\right. \\
& -\sum_{i j} \Lambda_{i j}\left(\left\langle\psi_{i} \mid \psi_{j}\right\rangle-\delta_{i j}\right)-\lambda \sum_{j}\left(\sum_{j} P_{j}-1\right) \\
& \left.-\sum_{\alpha \beta} h_{\alpha \beta}^{\prime}\left(\sum_{j} P_{j}\left\langle\psi_{j}\left|\hat{c}_{\beta}^{\dagger} \hat{c}_{\alpha}\right| \psi_{j}\right\rangle-\rho_{\alpha \beta}\right)\right\}
\end{aligned}
$$

where $\boldsymbol{h}^{\prime}$ is the Lagrange multiplier matrix which enforces the density matrix constraint for the many particle wavefunctions, as defined in Eq. 2.74). The reduced density matrix functional $F_{\beta}^{\hat{W}}[\boldsymbol{\rho}]$ defined in this way is a universal quantity that only depends on the interaction tensor $\hat{\boldsymbol{W}}$. This two step optimization leads to putting all the complexity the many-particle wave-functions in the reduced density matrix functional.

The grand potential in the occupation and natural orbitals representation has the 
form,

$$
\begin{aligned}
\Omega_{\beta}^{\hat{\boldsymbol{h}}+\hat{\boldsymbol{W}}}= & \min _{f_{n} \in[0,1],\left|\phi_{n}\right\rangle}\left\{\sum_{n} f_{n}\left\langle\phi_{n}|\hat{h}| \phi_{n}\right\rangle-\mu \sum_{n} f_{n}+F_{\beta}^{\hat{W}}\left[\sum_{n} f_{n}\left|\phi_{n}\right\rangle\left\langle\phi_{n}\right|\right]\right. \\
& \left.-\sum_{m n} \Lambda_{m n}\left(\left\langle\phi_{n} \mid \phi_{m}\right\rangle-\delta_{m n}\right)\right\}
\end{aligned}
$$

where the Lagrange multipliers $\boldsymbol{\Lambda}$ are used to enforce the orthogonality of the natural orbitals.

The density matrix functional obtained from the constrained wave function approach described above is a convex function

$$
F_{\beta}^{\hat{W}}\left[(1-\lambda) \boldsymbol{\rho}^{A}+\lambda \boldsymbol{\rho}^{B}\right] \leq(1-\lambda) F_{\beta}^{\hat{W}}\left[\boldsymbol{\rho}^{A}\right]+\lambda F_{\beta}^{\hat{W}}\left[\boldsymbol{\rho}^{B}\right]
$$

and therefore it satisfies the true minimum principle. The proof of the convexity can be found in the work of Blöchl et al [48. The true minimum principle comes in handy for numerical purpose too as it is far easier to search for a minima in a multi-dimensional space than looking for a stationary point.

The formulation of density matrix functional until now is exact and it has the same complexity as that of the grand potential for the many body system which suffers from the exponential increase in the many body Hilbert space with the system size. In the work of [68], first attempts were made to evaluate density matrix functional within this constrained search formalism. They employed local approximation to study finite Hubbard chains and were able to reproduce ground state energy, occupations and spin correlations to considerable accuracy.

\subsubsection{Green's function based method}

The link from rDMFT to many-body perturbation theory and the Green's function was provided by Blöchl et al. in 2013 [48.

They derived the density-matrix functional from the Kadanoff-Baym functional and thereby bridged the gap between density functional community and Green's function based many-body community. This construction as we will show, leads to a convex functional and therefore provides a minimum principle. The construction will also be a starting point for further discussion in Chapter 5.

The construction starts with the Luttinger-Ward identity [70] where the grand canonical potential $\Omega_{\beta}^{\hat{h}+\hat{W}}$ is obtained as a stationary point of Kadanoff-Baym functional $\Psi_{\beta}^{K B}[\boldsymbol{G}, \boldsymbol{\Sigma}, \boldsymbol{h}, \hat{\boldsymbol{W}}]$ as,

$$
\Omega_{\beta}^{\hat{\boldsymbol{h}}+\hat{\boldsymbol{W}}}=\operatorname{stat}_{\boldsymbol{G}, \boldsymbol{\Sigma}} \Psi_{\beta}^{K B}[\boldsymbol{G}, \boldsymbol{\Sigma}, \boldsymbol{h}, \hat{\boldsymbol{W}}]
$$


where the Kadanoff-Baym functional $\Psi_{\beta}^{K B}[\boldsymbol{G}, \boldsymbol{\Sigma}, \boldsymbol{h}, \hat{\boldsymbol{W}}]$ in its full glory has the form,

$$
\begin{aligned}
\Psi_{\beta}^{K B}[\boldsymbol{G}, \boldsymbol{\Sigma}, \boldsymbol{h}, \hat{\boldsymbol{W}}] & =\Phi^{\hat{\boldsymbol{W}}}[\boldsymbol{G}]-\frac{1}{\beta} \operatorname{Tr}\left\{\ln \left(\mathbf{1}+e^{-\beta(\boldsymbol{h}-\mu \mathbf{1})}\right)\right\} \\
& -\frac{1}{\beta} \sum_{\omega_{n}} \operatorname{Tr}\left\{\ln \left(\mathbf{1}-\frac{\mathbf{1}}{\left(i \omega_{n}+\mu\right) \mathbf{1}-\boldsymbol{h}} \boldsymbol{\Sigma}\left(i \omega_{n}\right)\right)+\boldsymbol{\Sigma}\left(i \omega_{n}\right) \boldsymbol{G}\left(i \omega_{n}\right)\right\}
\end{aligned}
$$

One difference in the above expression from the Luttinger-Ward identity of [70] is that, the grand potential of the non-interacting part i.e. $\frac{1}{\beta} \operatorname{Tr}\left\{\ln \left(\mathbf{1}+e^{-\beta(\boldsymbol{h}-\mu \mathbf{1})}\right)\right\}$, is separated.

The quantity $\Phi^{\hat{\boldsymbol{W}}}[\boldsymbol{G}]$ in Eq. 2.79 is the Luttinger-Ward functional that can be evaluated perturbatively using the skeleton diagrams as discussed in Appendix C.

\section{Properties of Luttinger-Ward functional}

The Luttinger-Ward functional by construction is a universal functional i.e. it only depends on the interaction $\hat{\boldsymbol{W}}$ and not on the one-particle part of the Hamiltonian. Secondly, the first order variation of Luttinger-Ward functional with respect to the Green's function provides the self-energy as,

$$
\begin{aligned}
\Phi^{\hat{\boldsymbol{W}=0}}[\boldsymbol{G}] & =0 \\
\frac{\delta \Phi^{\hat{\boldsymbol{W}}}[\boldsymbol{G}]}{\delta G_{\alpha \beta}\left(i \omega_{n}\right)} & =\frac{1}{\beta} \Sigma_{\beta \alpha}\left(i \omega_{n}\right)
\end{aligned}
$$

\section{Connection between $F_{\beta}^{\hat{W}}[\boldsymbol{\rho}]$ and $\Phi^{\hat{W}}[\boldsymbol{G}]$}

The connection between Kadanoff-Baym functional $\Psi_{\beta}^{K B}[\boldsymbol{G}, \boldsymbol{\Sigma}, \boldsymbol{h}, \hat{\boldsymbol{W}}]$ and the density matrix functional was derived using an important property [48] of KadanoffBaym functional which states, that it is invariant under a change $\boldsymbol{\Delta}$ of the noninteracting Hamiltonian $\boldsymbol{h}$ as,

$$
\Psi_{\beta}^{K B}[\boldsymbol{G}, \boldsymbol{\Sigma}, \boldsymbol{h}, \hat{\boldsymbol{W}}]=\Psi_{\beta}^{K B}[\boldsymbol{G}, \boldsymbol{\Sigma}+\boldsymbol{\Delta}, \boldsymbol{h}-\boldsymbol{\Delta}, \hat{\boldsymbol{W}}]+\frac{1}{\beta} \sum_{\omega_{n}} e^{i \omega_{n} 0^{+}} \operatorname{Tr}\left(\boldsymbol{G}\left(i \omega_{n}\right) \boldsymbol{\Delta}\right)
$$

The invariance of Kadanoff-Baym functional in Eq.2.82 holds not just when the stationary conditions are satisfied, but it is valid point per point.

Using this invariance with respect to the change $\Delta$ in the one-particle Hamiltonian $\boldsymbol{h}$, the connection to the density matrix $\boldsymbol{\rho}$ was made possible. The connection proceed with the construction of a one-particle density matrix $\boldsymbol{\rho}[\boldsymbol{G}]$ dependent 
Hamiltonian $\overline{\boldsymbol{h}}[\boldsymbol{\rho}[\boldsymbol{G}]]$ that can be constructed using the freedom of $\boldsymbol{\Delta}[\boldsymbol{\rho}[\boldsymbol{G}]]$ as,

$$
\overline{\boldsymbol{h}}[\boldsymbol{\rho}[\boldsymbol{G}]]=\boldsymbol{h}-\boldsymbol{\Delta}[\boldsymbol{\rho}[\boldsymbol{G}]]
$$

where the one particle density matrix $\boldsymbol{\rho}[\boldsymbol{G}]$ is obtained from the given Green's function $\boldsymbol{G}\left(i \omega_{n}\right)$ as,

$$
\boldsymbol{\rho}[\boldsymbol{G}]=\frac{1}{\beta} \sum_{\omega_{n}} \mathrm{e}^{i \omega_{n} 0^{+}} \boldsymbol{G}\left(i \omega_{n}\right)
$$

The non-interacting Hamiltonian $\overline{\boldsymbol{h}}[\boldsymbol{\rho}[\boldsymbol{G}]]$ is obtained by inverting the corresponding expression for the one particle reduced density matrix $\boldsymbol{\rho}$,

$$
\boldsymbol{\rho}=\left[\mathbf{1}+\mathrm{e}^{\beta(\overline{\boldsymbol{h}}-\mu \mathbf{1})}\right]^{-1}
$$

which when inverted has the form,

$$
\overline{\boldsymbol{h}}[\boldsymbol{\rho}]=\mu \mathbf{1}+\frac{1}{\beta} \ln \left[\frac{\mathbf{1}-\boldsymbol{\rho}}{\boldsymbol{\rho}}\right]
$$

This procedure helps in construction of one-particle reduced density matrix $\boldsymbol{\rho}$ dependent $\boldsymbol{\Delta}[\boldsymbol{\rho}]$ which in turn makes the expression on the right hand side of Eq. 2.82 density matrix dependent and Eq.2.82 attains the following form,

$$
\begin{aligned}
\Psi_{\beta, \mu}^{K B}[\boldsymbol{G}, \boldsymbol{\Sigma}, \boldsymbol{h}, \hat{W}] & =\sum_{\omega_{n}} f_{n}\left\langle\psi_{n}|\hat{h}| \psi_{n}\right\rangle+\frac{1}{\beta} \sum_{n}\left(f_{n} \ln \left(f_{n}\right)+\left(1-f_{n}\right) \ln \left(1-f_{n}\right)\right) \\
& -\mu \sum_{n} f_{n}+\Phi_{\beta}^{\hat{W}}[\boldsymbol{G}, \hat{W}] \\
& -\frac{1}{\beta} \sum_{\omega_{n}} e^{i \omega_{n} 0^{+}} \operatorname{Tr}\left\{\ln \left[\mathbf{1}-\left(\left(i \omega_{n}+\mu\right) \mathbf{1}-\overline{\boldsymbol{h}}\right)^{-1}\left(\boldsymbol{h}+\boldsymbol{\Sigma}\left(i \omega_{n}\right)-\overline{\boldsymbol{h}}\right)\right]\right. \\
& \left.+\left(\boldsymbol{h}+\boldsymbol{\Sigma}\left(i \omega_{n}\right)-\overline{\boldsymbol{h}}\right) \boldsymbol{G}\left(i \omega_{n}\right)+\boldsymbol{G}\left(i \omega_{n}\right)(\overline{\boldsymbol{h}}-\boldsymbol{h})\right\} \\
& +\sum_{n} f_{n}\left\langle\psi_{n}|(\hat{\bar{h}}-\hat{h})| \psi_{n}\right\rangle
\end{aligned}
$$

where the first and the last line combined of Eq.(2.87) comes from the fact that the grand canonical potential of the non-interacting Hamiltonian $\overline{\boldsymbol{h}}$ can be written in terms of occupations $f_{n}$ and natural orbitals $\left|\psi_{n}\right\rangle$ of one-particle reduced density matrix $\boldsymbol{\rho}$ as,

$$
\begin{aligned}
-\frac{1}{\beta} \operatorname{Tr}\left\{\ln \left(1+\mathrm{e}^{-\beta(\overline{\boldsymbol{h}}-\mathbf{1} \mu)}\right)\right\} & =\sum_{n} f_{n}\left\langle\psi_{n}|\hat{\bar{h}}| \psi_{n}\right\rangle-\mu \sum_{n} f_{n} \\
& +\frac{1}{\beta} \sum_{n}\left[f_{n} \ln \left(f_{n}\right)+\left(1-f_{n}\right) \ln \left(1-f_{n}\right)\right]
\end{aligned}
$$


Blöchl et al. [48] performed the stationary point search in Eq.2.78 in two steps.

1. In the first step, the stationary point search is performed in the space of Green's function $\boldsymbol{G}\left(i \omega_{n}\right)$ and self-energy $\boldsymbol{\Sigma}\left(i \omega_{n}\right)$ which produces a given one-particle reduced density matrix $\boldsymbol{\rho}$ i.e. it satisfies the constraint of Eq. 2.84). This step leads to a density matrix functional $F^{\hat{\boldsymbol{W}}}[\boldsymbol{\rho}]$ which has the form,

$$
\begin{aligned}
F^{\hat{\boldsymbol{W}}}[\boldsymbol{\rho}] & =\frac{1}{\beta} \operatorname{Tr}[\boldsymbol{\rho} \ln (\boldsymbol{\rho})+(\mathbf{1}-\boldsymbol{\rho}) \ln (\mathbf{1}-\boldsymbol{\rho})] \\
& +\underset{\boldsymbol{h}^{\prime}}{\operatorname{stat}} \operatorname{stat}_{\boldsymbol{G}}\left\{\Phi_{\beta}^{\hat{\boldsymbol{W}}}[\boldsymbol{G}, \hat{W}]\right. \\
& -\frac{1}{\beta} \sum_{\nu} \operatorname{Tr}\left\{\ln \left[\mathbf{1}-\left(\left(i \omega_{\nu}+\mu\right) \mathbf{1}-\overline{\boldsymbol{h}}\right)^{-1}\left(\boldsymbol{h}^{\prime}+\boldsymbol{\Sigma}\left(i \omega_{\nu}\right)-\overline{\boldsymbol{h}}\right)\right]\right. \\
& +\left(\boldsymbol{h}^{\prime}+\boldsymbol{\Sigma}\left(i \omega_{\nu}\right)-\overline{\boldsymbol{h}}\right) \boldsymbol{G}\left(i \omega_{\nu}\right) \\
& \left.\left.-\left[\boldsymbol{G}\left(i \omega_{\nu}\right)-\left(\left(i \omega_{\nu}+\mu\right) \mathbf{1}-\overline{\boldsymbol{h}}\right)^{-1}\right]\left(\boldsymbol{h}^{\prime}-\overline{\boldsymbol{h}}\right)\right\}\right\}
\end{aligned}
$$

The last part in Eq. 2.89) vanishes when the Green's function $\boldsymbol{G}\left(i \omega_{n}\right)$ obeys the density matrix constraint of Eq. (2.84). This constraint is enforced using the Lagrange multiplier $\boldsymbol{h}^{\prime}$. The remaining part of Eq.(2.89) is the Kadanoff-Baym functional for a many body system with one particle density matrix dependent non-interacting Hamiltonian $\overline{\boldsymbol{h}}$ and the self-energy replaced by $\boldsymbol{h}^{\prime}+\boldsymbol{\Sigma}-\overline{\boldsymbol{h}}$.

The density matrix functional $F^{\hat{\boldsymbol{W}}}[\boldsymbol{\rho}]$ defined in Eq. 2.89$]$ is also a universal functional which is independent of the non-interacting Hamiltonian $\boldsymbol{h}$. This representation of the density matrix functional provides a true minimum principle and also provides a direct link between the density-functional community and the Green's function based many-body community.

2. The second step is to evaluate the grand canonical potential $\Omega_{\beta}^{\hat{\boldsymbol{h}}+\hat{\boldsymbol{W}}}$ by performing a minimization in the space of N-representable one-particle reduced density matrices as,

$$
\begin{aligned}
\Omega_{\beta}^{\hat{\boldsymbol{h}}+\hat{\boldsymbol{W}}}= & \min _{f_{n} \in[0,1],\left|\phi_{n}\right\rangle}\left\{\sum_{n} f_{n}\left\langle\phi_{n}|\hat{h}| \phi_{n}\right\rangle-\mu \sum_{n} f_{n}+F_{\beta}^{\hat{W}}\left[\sum_{n} f_{n}\left|\phi_{n}\right\rangle\left\langle\phi_{n}\right|\right]\right. \\
& \left.-\sum_{m n} \Lambda_{m n}\left(\left\langle\phi_{n} \mid \phi_{m}\right\rangle-\delta_{m n}\right)\right\}
\end{aligned}
$$


where we have used the eigen-decomposition of one particle reduced density matrix.

\section{Stationary point search}

The expression for density matrix functional $F_{\beta}^{\hat{W}}[\boldsymbol{\rho}]$ in Eq. $(2.89)$ involves a stationary point search in the space of Green's function $\boldsymbol{G}\left(i \omega_{n}\right)$ and self-energy $\boldsymbol{\Sigma}\left(i \omega_{n}\right)$. The stationary conditions obtained from Eq. 2.89) are,

- The stationarity with respect to the Green's function $\boldsymbol{G}\left(i \omega_{n}\right)$ leads to,

$$
\frac{1}{\beta} \boldsymbol{\Sigma}\left(i \omega_{n}\right)=\frac{\delta \Phi^{\hat{\boldsymbol{W}}}[\boldsymbol{G}]}{\delta G\left(i \omega_{n}\right)}
$$

- The stationarity with respect to the self energy $\boldsymbol{\Sigma}\left(i \omega_{n}\right)$ leads to the Dyson equation,

$$
\boldsymbol{G}\left(i \omega_{n}\right)=\frac{\mathbf{1}}{\left(i \omega_{n}+\mu\right) \mathbf{1}-\boldsymbol{h}^{\prime}-\boldsymbol{\Sigma}\left(i \omega_{n}\right)}
$$

- The stationarity with respect to the Lagrange multiplier $\boldsymbol{h}^{\prime}$ leads to the density matrix constraint,

$$
\boldsymbol{\rho}=\frac{1}{\beta} \sum_{\omega_{n}} \mathrm{e}^{i \omega_{n} 0^{+}} \boldsymbol{G}\left(i \omega_{n}\right)
$$

It is not an extremum search and therefore we cannot employ the usual Car-Parinello procedure of Chapter 3 . One has to rely on traditional self-consistency schemes for the stationary point search as described in Blöchl et al. [48].

The full self-consistency scheme has not been implemented yet. In the current work, we want to provide a framework for an approximate scheme where we can completely avoid the internal optimization in an exponentially large space of Green's function and self-energy. To this direction, in Chapter 5, we will discuss different approximate schemes to obtain an algebraic expression for the density matrix functional.

\subsubsection{Parametrized density matrix functionals}

The evaluation of density matrix functional from the constrained search formalism of wave-function [68] and the statinary point search of Green's function based methods [48] is very recent. These methods are still in the development stage and Chapter 5 of this thesis will provide the framework for first controlled approximations in the direction of Green's function based methods.

Three decades ago the advancement of density matrix functional theory proceeded in the direction of density functional approaches i.e. through constructing approximations for density matrix functional $F_{\beta}^{\hat{\boldsymbol{W}}}[\boldsymbol{\rho}]$ based on physical justifications. These 
methods avoid complicated many-body calculations and proceed towards providing algebraic expression of the density matrix functional motivated through certain physical properties the functionals should obey. This approach provides a direct mapping of the form,

$$
\boldsymbol{\rho} \rightarrow F_{\beta}^{\hat{W}}[\boldsymbol{\rho}]
$$

An enormous amount of scientific advancement has taken in the direction of approximate density matrix functionals which are parameter based and are rather uncontrolled in the sense of treatment of correlation effects. To this date there has been several proposals for the approximate density matrix functionals. In this section we review a small number of density matrix functionals, a class of which will be investigated for its validity in Chapter 4.

\section{Review}

The advancements in the direction of reduced density matrix functional theory started to address the bond dissociation of diatomic molecules. It is an important problem in the electronic structure calculations.

The hydrogen molecule $\mathrm{H}_{2}$ is a simple prototype for the electron bond pair. Although the exact wave function of infinitely displaced hydrogen molecule is known exactly, it is still not described exactly through density functional approaches. The $\mathrm{H}_{2}$ dissociation problem is a long-standing problem in the field of density functional theory. Density-functional theory (DFT), with exchange-correlation functional approximated by the local density approximation (LDA) or the generalized-gradient approximation (GGA), fails in estimating the energy of stretched hydrogen molecule. Spin polarised DFT however performs better in estimating the energy but produces incorrect ground state. It produces broken spin symmetry state.

The inherent deficiency in the different approximations for the density functionals is well explained by E J Baerands et al. in [71] and [72] through hole function based analysis. They also provided an alternative approximation which fixes the deficiency and enables the functional to describe the bond dissociation problem of $\mathrm{H}_{2}$ molecule exactly within density functional based approcah.

The approximation goes into constructing suitable hole function function $h(\vec{r}, \vec{r})$ that allows to express the two-particle density $n^{(2)}\left(\vec{r}, \overrightarrow{r^{\prime}}\right)$ in the form

$$
n^{(2)}\left(\vec{r}, \overrightarrow{r^{\prime}}\right)=n(\vec{r}) n\left(\overrightarrow{r^{\prime}}\right)+n(\vec{r}) h\left(\vec{r}, \overrightarrow{r^{\prime}}\right)
$$

Note that the interaction-strength averaged hole function is used in DFT, while in rDMFT, the hole function at full interaction strength is of interest.

The hole function integrates to minus one (See Eq. (2.44)), and it is always negative [73]. These conditions constrain the shape of the hole function strongly, so that the exchange-correlation energy can be predicted reasonably well already with simple assumptions about the hole function. 
The hole function describes the exchange correlation energy $U_{x c}[\boldsymbol{\rho}]$ as,

$$
\begin{aligned}
F_{\beta}^{\hat{\boldsymbol{W}}}[\boldsymbol{\rho}] & =\frac{1}{\beta} \operatorname{Tr}[\boldsymbol{\rho} \ln (\boldsymbol{\rho})+(\mathbf{1}-\boldsymbol{\rho}) \ln (\mathbf{1}-\boldsymbol{\rho})]+\frac{1}{2} \int d^{3} r \int d^{3} r^{\prime} \frac{n^{(2)}\left(\vec{r}, \overrightarrow{r^{\prime}}\right)}{4 \pi \epsilon_{0}\left|\vec{r}-\overrightarrow{r^{\prime}}\right|} \\
& =\frac{1}{\beta} \operatorname{Tr}[\boldsymbol{\rho} \ln (\boldsymbol{\rho})+(\mathbf{1}-\boldsymbol{\rho}) \ln (\mathbf{1}-\boldsymbol{\rho})]+\frac{1}{2} \int d^{3} r \int d^{3} r^{\prime} \frac{n(\vec{r}) n\left(\overrightarrow{r^{\prime}}\right)}{4 \pi \epsilon_{0}\left|\vec{r}-\overrightarrow{r^{\prime}}\right|} \\
& +\frac{1}{2} \int d^{3} r n(\vec{r}) \int d^{3} r^{\prime} \frac{h\left(\vec{r}, \vec{r}^{\prime}\right)}{4 \pi \epsilon_{0}\left|\vec{r}-\overrightarrow{r^{\prime}}\right|} \\
& =\frac{1}{\beta} \operatorname{Tr}[\boldsymbol{\rho} \ln (\boldsymbol{\rho})+(\mathbf{1}-\boldsymbol{\rho}) \ln (\mathbf{1}-\boldsymbol{\rho})]+E_{H}[\boldsymbol{\rho}]+U_{x c}[\boldsymbol{\rho}]
\end{aligned}
$$

In the Hartree-Fock approximation, the hole function has the form

$$
h\left(\vec{r}, \overrightarrow{r^{\prime}}\right)=\frac{-1}{n(\vec{r})} \sum_{m, n} f_{m} f_{n} \sum_{\sigma, \sigma^{\prime}} \phi_{m}^{*}(\vec{x}) \phi_{n}(\vec{x}) \phi_{n}^{*}\left(\overrightarrow{x^{\prime}}\right) \phi_{m}\left(\overrightarrow{x^{\prime}}\right)
$$

As a consequence of the orthonormality of the natural orbitals, the sum rule Eq. (2.44) is obtained as

$$
\int d^{3} r^{\prime} h\left(\vec{r}, \overrightarrow{r^{\prime}}\right)=\frac{-1}{n(\vec{r})} \sum_{n \sigma} f_{n}^{2} \phi_{n}^{*}(\vec{x}) \phi_{n}(\vec{x})=-1
$$

The sum-rule is fulfilled exactly, when $f_{n}^{2}=f_{n}$ that is for integer occupations. For fractional occupations, however, the Hartree-Fock expression violates the sum-rule.

The exchange-correlation term in the Hartree-Fock approximation is

$$
\begin{aligned}
U_{x c}^{H F}[\boldsymbol{\rho}]= & -\frac{1}{2} \sum_{m, n} f_{m} f_{n} \sum_{\alpha \beta \gamma \delta} U_{\alpha \beta, \delta \gamma} \\
& \times\left\langle\chi_{\gamma} \mid \phi_{m}\right\rangle\left\langle\phi_{m} \mid \chi_{\alpha}\right\rangle\left\langle\chi_{\delta} \mid \phi_{n}\right\rangle\left\langle\phi_{n} \mid \chi_{\beta}\right\rangle .
\end{aligned}
$$

In the exact scenario, when the two hydrogen atoms are separated by large distance, the reference electron sees an unscreened nucleus, as described in the total hole on the lower panel of Fig. 2.1. The Fermi/Fock hole, on the other hand, leaves an electron density which screens the nucleus. Therefore the Hartree-Fock potential cannot describe the dissociation problem of hydrogen molecule exactly.

Similarly LDA (local density approximation) and the GGA (generalized gradient approximation) also fail in describing the dissociation problem of $\mathrm{H}_{2}$. The problem with LDA and GGA lies in the fact they depend exclusively on the local information such as density and the derivatives of density and therefore can never build the exact delocalised (Right most plot in upper panel of Fig. 2.1) exchange correlation hole. Therefore one has to move beyond local densities and their derivatives, and also include natural orbitals specifically to the exchange correlation energy. 


\section{Fermi hole + Coulomb hole $=$ total hole}
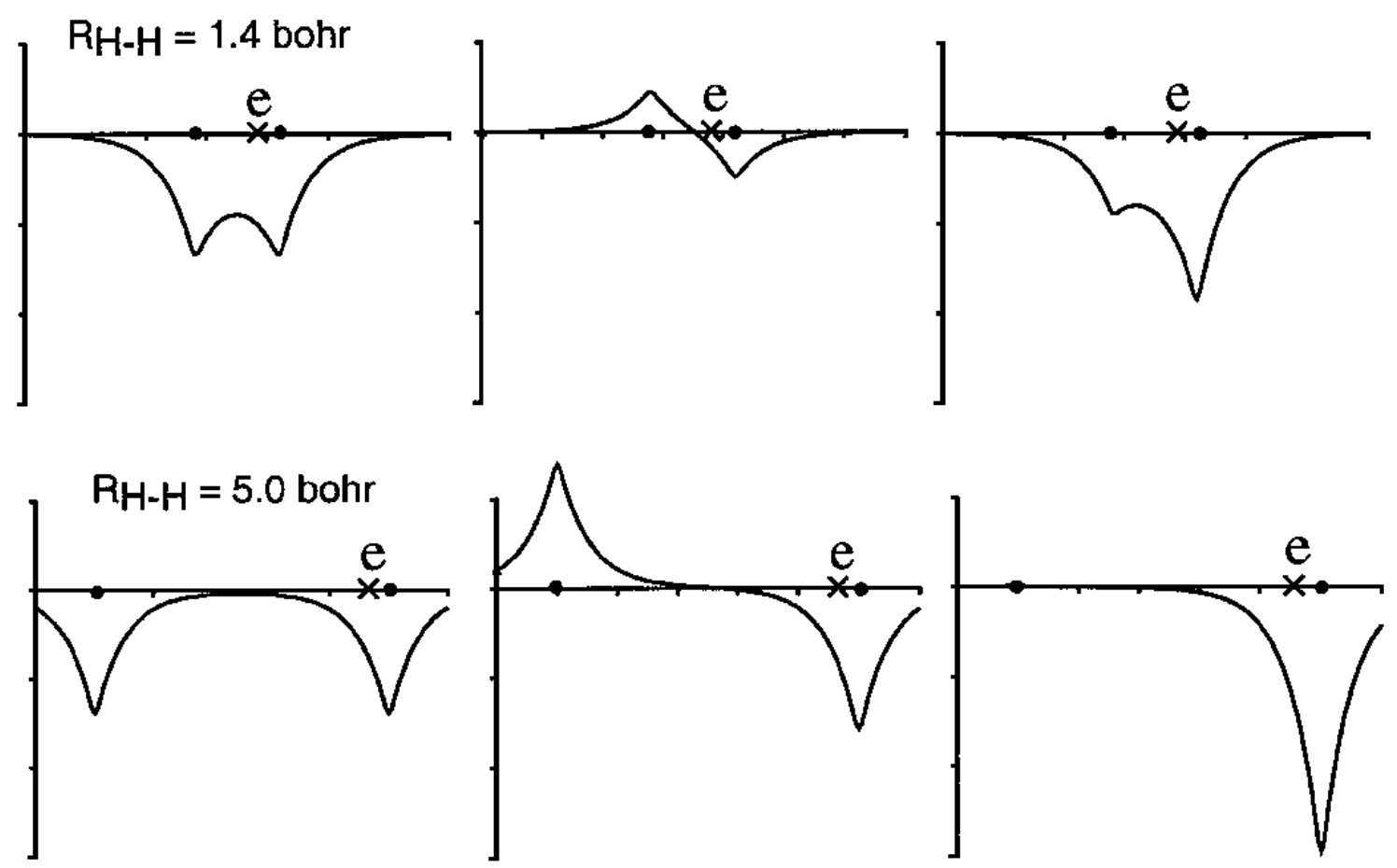

Figure 2.1: . Fermi hole, Coulomb hole and the total hole in the hydrogen molecule at equilibrium distance $R=1.4$ and $R=5.0$ bohr. The reference electron is placed at $0.3 \mathrm{bohr}$ to the left of right hydrogen atom. Figure taken from [71]

Baerands et al suggested following approximation for the exchange correlation hole $h\left(\vec{r}, \vec{r}^{\prime}\right)$ as,

$$
h\left(\vec{r}, \overrightarrow{r^{\prime}}\right)=\frac{-1}{n(\vec{r})} \sum_{m, n} f_{m}^{\frac{1}{2}} f_{n}^{\frac{1}{2}} \sum_{\sigma, \sigma^{\prime}} \phi_{m}^{*}(\vec{x}) \phi_{n}(\vec{x}) \phi_{n}^{*}\left(\overrightarrow{x^{\prime}}\right) \phi_{m}\left(\overrightarrow{x^{\prime}}\right) .
$$

This particular form of exchange correlation hole makes the exchange correlation energy $U_{x c}[\rho]$, natural orbitals dependent and also the dependence on occupation is linear unlike quadratic as in Hartree-Fock approximation. This particular form of exchange correlation energy describes correlations remarkably well for two electrons molecules and it describes the dissociation problem of $\mathrm{H}_{2}$ molecule exactly.

This particular form of exchange correlation hole in Eq. 2.100) was also suggested by Müller [50] in 1984. Müller suggested following form of the exchange-correlation 
hole,

$$
h\left(\vec{r}, \overrightarrow{r^{\prime}}\right)=\frac{-1}{n(\vec{r})} \sum_{m, n} f_{m}^{\frac{1}{2}+p} f_{n}^{\frac{1}{2}-p} \sum_{\sigma, \sigma^{\prime}} \phi_{m}^{*}(\vec{x}) \phi_{n}(\vec{x}) \phi_{n}^{*}\left(\overrightarrow{x^{\prime}}\right) \phi_{m}\left(\overrightarrow{x^{\prime}}\right) .
$$

This particluar form of the hole function also satisfies the sum rule of Eq. (2.44), even for the fractional occupations as,

$$
\begin{aligned}
\int d^{3} r^{\prime} h\left(\vec{r}, \overrightarrow{r^{\prime}}\right) & =\frac{-1}{n(\vec{r})} \sum_{m, n \sigma} f_{m}^{\frac{1}{2}+p} f_{n}^{\frac{1}{2}-p} \phi_{m}^{*}(\vec{x}) \phi_{n}(\vec{x}) \delta_{m n} \\
& =-\frac{1}{n(\vec{r})} \sum_{m \sigma} f_{m} \phi_{m}^{*}(\vec{x}) \phi_{m}(\vec{x}) \\
& =-1
\end{aligned}
$$

where we have used the orthonormality of naturals orbitals as,

$$
\sum_{\sigma} \int d^{3} r\left\langle\phi_{m} \mid \vec{r}, \sigma\right\rangle\left\langle\vec{r}, \sigma \mid \phi_{n}\right\rangle=\delta_{m n}
$$

Although with the Müller hole function the two particle density matrix $n^{(2)}\left(\vec{r}, \vec{r}^{\prime}\right)$ does not satisfy the Pauli principle of Eq. 2.45), i.e.,

$$
n^{(2)}(\vec{r}, \vec{r}) \neq 0
$$

Given the fact that for a finite value of $p \geq 0$, the fractional occupation $f_{m}$, satisfies,

$$
f_{m}^{\frac{1}{2}+p} \geq f_{m}
$$

and therefore it can be shown that for a fixed finite value of $p$, the two particle density matrix follows the condition,

$$
n^{(2)}(\vec{r}, \vec{r}) \leq 0
$$

Müller [50] showed that the two particle density at the same position in Eq.(2.106), has a maxima at $p=0$ and therefore Pauli principle is least violated for $p=0$. This is the value used in nearly all applications.

The Müller functional was however found to be over-correlating for many systems [74, 75, 76] and therefore several repulsive corrections were itroduced later. In the following discussion we briefly mention few such correction. 


\section{Corrections to Müller functional}

\section{Self interaction corrections}

In 1998, Goedecker and Umrigar [52] improved on the Müller functional by removing the self-interactions terms of the natural orbitals from the Hartree and the exchange correlation functional. They replaced the square root dependence on occupations in the exchange-correlation energy $U_{\mathrm{xc}}$ as,

$$
f_{m}^{\frac{1}{2}} f_{n}^{\frac{1}{2}} \rightarrow f_{m}^{\frac{1}{2}} f_{n}^{\frac{1}{2}}-\delta_{m n}\left(f_{m}-f_{m}^{2}\right)
$$

This particular choice of two particle density removes orbitals self-interaction but does not completely remove the electron self-interaction due to fractional occupations. They performed calculations for atoms like H, He, Li, Be, C and $\mathrm{Ne}$ and concluded that their proposed correction to Müller functional provides more accurate energies and the charge densities than the LDA, GGA and Hartree-Fock approximations.

Although the removal of self interaction terms of the natural orbitals improves on the correlation energies, it does fail dramatically at the dissociation limit. For homogenous electron gas, it fails to even reproduce the correct correlation energies [76].

\section{BBCn corrections}

In 2005, Gritsenko et al. [51], proposed three physically motivated repulsive corrections (termed as BBCn corrections) to compensate for the over correlating effect of the Müller functional. The correction to BB or the Müller functional [50] involves identification of weak and strongly occupied orbitals during the course of optimization.

These corrections were tested on several diatomic molecules like $\mathrm{H}_{2}, \mathrm{LiH}, \mathrm{BH}$, $\mathrm{Li}_{2}$, $\mathrm{HF}$ and the potential energy landscape of these molecules obtained from different corrections were compared with the exact obtained from full configuration interaction method.

In Fig. 2.2, the potential energy curve of $\mathrm{H}_{2}$ molecule is plotted as a function of inter-atomic distance. BB functional or the Müller functional on the other hand over estimates the energy, but becomes exact at large distances. BBCn correction [51] reproduces the CI curve for $\mathrm{H}_{2}$ excellently and at large distances converges faster to the CI curve. Results for different molecules can be found in [51].

BBC3 correction to the Müller functional is the most precise functional for the molecular systems which reproduces correlation energies that are around $17-20 \%$ from the reference results obtained from more accurate couple cluster methods [77] 


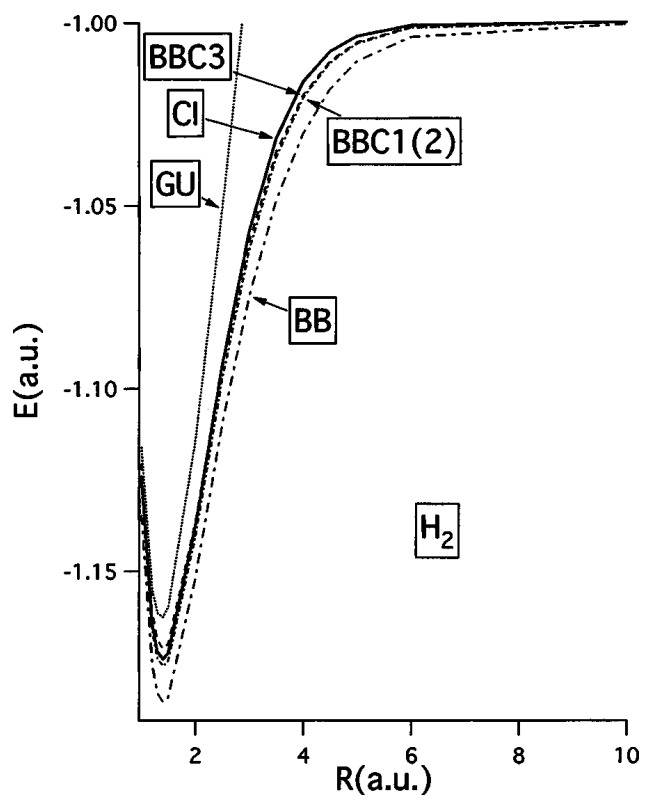

Figure 2.2: Energy curve for $\mathrm{H}_{2}$ molecule obtained from different corrections to the Müller functional. These curves are compared with exact CI curve. Figure taken from [51].

\section{ML functional}

Marques et al. in 2008 [54], proposed a different class of density matrix functional, which is purely empirical and the exchange-correlation $U_{\mathrm{xc}}$ dependence on the occupations is written as a Pade approximant as,

$$
f_{m} f_{n} \frac{a_{0}+a_{1} f_{m} f_{n}+a_{2}\left(f_{m} f_{n}\right)^{2}+\ldots}{\left.1+b_{1} f_{m} f_{n}+b_{2} f_{m} f_{n}\right)^{2}+\ldots}
$$

where $a_{0}, a_{1}, b_{1}$ etc. are the Pade coefficients which are obtained by optimizing an objective function

$$
\bar{\delta}=100 \sqrt{\frac{1}{N} \sum_{i=1}^{N}\left(\frac{E_{c}^{\mathrm{ML}}-E_{c}^{\mathrm{ref}}}{E_{c}^{\mathrm{ref}}}\right)^{2}}
$$

where $\mathrm{N}$ is the number of molecules in the set and $E_{c}^{\mathrm{ref}}$ is the ground state obtained from accurate couple cluster theory. The molecules used in the reference set can have wide range of reference energies (mili-Hartree to order of Hartree). Using the ML functional, the correlation energies obtained for typical molecules were $60 \%$ more accurate than the best functional available i.e. the BBC3 functional [51]. 


\section{Power functional}

Most of the reduced density matrix functional studies were concentrated on atoms and molecules. In 2008, Sharma et al. 53 proposed a Müller like functional and implemented it on the extended system, specifically the strongly correlated materials like transition metal oxides (TMOs) which are known to be Mott insulators. The density functional studies on TMOs produces metallic ground state in a spin unpolarized calculations. In spin-polarised calculations density functional studies produces a small band gap, but that is largely due to anti-ferromagnetic ordering.

Sharma et al. [53] proposed a Müller like functional where the square root dependence on the occupations in exchange-correlation energy $U_{\mathrm{xc}}$ was replaced by,

$$
f_{m}^{\frac{1}{2}} f_{n}^{\frac{1}{2}} \rightarrow f_{m}^{\alpha} f_{n}^{\alpha}
$$

where tuning the parameter from $\alpha=\frac{1}{2}$ to $\alpha=1$, one can interpolate between Müller or BB functional to Hartree-Fock functional respectively. Using a tangent method [53] for the calculation of band gap, power functional predicted band gaps for $\mathrm{Si}$ and $\mathrm{NiO}$, which deviated from experimental band gaps by $9.5 \%$. The most advanced BBC3 [51] and Piris natural orbitals functional (PNOF) [78] which yield results for molecular systems with accuracy comparable to more accurate coupled cluster methods, fail miserably for extended systems. Band insulator like Si and Mott insulator such as $\mathrm{NiO}$, turns out to be metallic when studied with BBC3 and PNOF functionals.

Lathiotakis et al. [79] also showed that power functional works equally well for homogenous electron gas and the finite systems. The $\mathrm{H}_{2}$ dissociation problem was reproduced with BBC3 level accuracy for $\alpha=0.525$.

In 2013, Sharma et al. [80] performed fully non-collinear calculations (spin unrestricted) calculation to study magnetic solids. They also developed the method to evaluate the dynamic quantity like spectral function within r-DMFT which is in principle a theory for evaluating only ground state properties. The density of states or the spectral function for transition metal oxides like $\mathrm{NiO}, \mathrm{MnO}, \mathrm{FeO}$ and $\mathrm{CoO}$ in the presence of anti-ferromagnetic order, were found to be in good agreement with dynamical mean field theory results.

\subsection{Test systems}

In our quest to benchmark the results obtained from different approximations, we will use simplified models which are either exactly solvable or have been studied extensively using numerically exact simulations.

One simplification which can be imposed on the general interaction tensor $\hat{W}$ in Eq. (2.24) is to restrict its elements over a finite cluster containing only small numbers of local spin orbitals $\alpha$. This restriction can be justified using the argu- 
ments of screening in strongly correlated electron systems. One such model which considers only local on-site Coulomb interaction was proposed independently by J. Hubbard [45], J Kanamori [46] and by M. C. Gutzwiller [44] in 1963. It is the most primitive model to study the properties of electrons in a solid. However simplistic, it does exhibits almost all interesting phenomena one observes in strongly correlated materials like Mott metal-insulator transition [45], magnetic ordering [81, 82, superconductivity [83, 84] etc.

The models are generally considered on translationally invariant lattices. Such lattices can be spanned completely through translating a unit cell. With an additional assumption of the interaction tensor $\hat{W}$ to be local, the knowledge of local spin orbitals in a unit cell is sufficient. Due to this simplication, the size of the interaction and the kinetic energy matrices are reduced considerably.

In order to keep ourself at the liberty of chosing a particular unit cell, we introduce a compact index for local basis set index $\alpha \equiv\left(\lambda_{\alpha}, \vec{T}_{\alpha}\right)$, where $\lambda_{\alpha}$ is the local orbital in the unit cell and $\vec{T}_{\alpha}$ is the identity of the unit cell containing the orbitals. In the special case of one atom per unit cell, the unit cell identity $\vec{T}$ is simply the position vector of the atom.

Using the compact notation defined above the sum in Eq. 2.22 translates as,

$$
\sum_{\alpha \beta \ldots} \rightarrow \sum_{\substack{\lambda_{\alpha}, \vec{T}_{\alpha} \\ \lambda_{\beta}, \vec{T}_{\beta}}}
$$

The most celebrated model is the one dimensional, one band Hubbard model. This model has one atom per unit cell with nearest neighbor hopping only. The local orbitals $\left\{\lambda_{\alpha}\right\}$ in the unit cell are the two spin orbitals $\{\uparrow, \downarrow\}$. Therefore we prefer to denote them as $\sigma_{\alpha}$. The hopping matrix elements has the following form,

$$
h_{\alpha, \beta}=-t\left(\delta_{T_{\beta}, T_{\alpha}+a}+\delta_{T_{\beta}, T_{\alpha}-a}\right) \delta_{\sigma_{\alpha} \sigma_{\beta}}
$$

where $t$ is positive and the onsite energies are chosen to be equal to zero. The lattice spacing is given by $a$.

The interaction tensor $W$ on the other hand is local in case of 1D Hubbard model which is given by a simple form as

$$
W_{\alpha \beta \gamma \delta}= \begin{cases}U, & \text { if } \alpha=\gamma \text { and } \beta=\delta \text { with } T_{\alpha}=T_{\beta} \\ 0, & \text { otherwise }\end{cases}
$$

With the simple form of hopping matrix in Eq.(2.112) and the interaction tensor in Eq. 2.113, the full Hamiltonian for 1D Hubbard model with one atom per unit cell 
has the form,

$$
\mathcal{H}=-t \sum_{T} \sum_{\sigma}\left(\hat{c}_{\sigma T}^{\dagger} \hat{c}_{\sigma T+A}+\text { h.c }\right)+\frac{U}{2} \sum_{T} \sum_{\sigma \sigma^{\prime}} \hat{c}_{T, \sigma}^{\dagger} \hat{c}_{T, \sigma^{\prime}}^{\dagger} \hat{c}_{T, \sigma^{\prime}} \hat{c}_{T, \sigma}
$$

The exact solution to one dimensional Hubbard model has been obtained from the Bethe ansatz [85, 86]. The one dimensional Hubbard model (also bipartite lattice) will be used as a test system for benchmarking the methods or approximations proposed in following chapters.

\subsubsection{Hubbard Dimer and the exact results}

In this section we present a part of the work of Kamil, E., Schade, R., Pruschke, T., and Blöchl, P. E., [56], concerning the exact results of the Hubbard dimer.

In density functional community the hydrogen $\mathrm{H}_{2}$ molecule is a protoype system for studying the chemical bond dissociation problem. The large interaction limit of the Hubbard dimer is essentially the same as bond dissociation limit of the hydrogen molecule. They differ only by the choice of energy scale. It will be essential to benchmark the results obtained from approximations proposed in subsequent chapters.

The Hubbard dimer is a simple system which has a one-particle basis with four spin orbitals $\left\{\left|\chi_{1, \uparrow}\right\rangle,\left|\chi_{1, \downarrow}\right\rangle,\left|\chi_{2, \uparrow}\right\rangle,\left|\chi_{2, \downarrow}\right\rangle\right\}$, one for each site and spin. The only nonzero matrix elements of the one-particle Hamiltonian

$$
h_{\alpha, \beta}=-t\left(1-\delta_{R_{\alpha}, R_{\beta}}\right) \delta_{\sigma_{\alpha}, \sigma_{\beta}}
$$

are those with orbitals having the same spin $\sigma_{\alpha}$ and $\sigma_{\beta}$ but different centers $R_{\alpha}$ and $R_{\beta}$. All nonzero elements have the value $-t$, where $t$ is positive. The orbital energies are chosen equal to zero.

Also the interaction tensor has a simple form, namely

$$
U_{\alpha \beta, \gamma \delta}=\left\{\begin{array}{ll}
U & \text { if } \alpha=\gamma, \beta=\delta \text { and } R_{\alpha}=R_{\beta} \\
0 & \text { otherwise }
\end{array} .\right.
$$

Thus, the Hamiltonian for the Hubbard dimer is

$$
\mathcal{H}=-\sum_{\sigma} t\left(\hat{c}_{1, \sigma}^{\dagger} \hat{c}_{2, \sigma}+\hat{c}_{2, \sigma}^{\dagger} \hat{c}_{1, \sigma}\right)+\hat{W}
$$


with the interaction

$$
\hat{W}=\frac{1}{2} \sum_{i=1}^{2} \sum_{\sigma, \sigma^{\prime}} U \hat{c}_{i, \sigma}^{\dagger} \hat{c}_{i, \sigma^{\prime}}^{\dagger} \hat{c}_{i, \sigma^{\prime}} \hat{c}_{i, \sigma} .
$$

The two-site Hubbard model, the Hubbard dimer, can be considered as model system for the correlation effects present in a chemical bond. If we denote the hopping parameter between the bonded atoms with $t$ and the on-site interaction strength with $U$, bond dissociation is described by the limit $t \rightarrow 0$ at constant $U$. Thus, the system evolves from a weakly correlated state into the strongly correlated limit $U / t \rightarrow \infty$ as the bond is broken. The large-interaction limit $U \rightarrow \infty$ of the Hubbard model differs from the bond-dissociation limit only by the choice of the energy scale.

\subsubsection{Exact energy and density matrix}

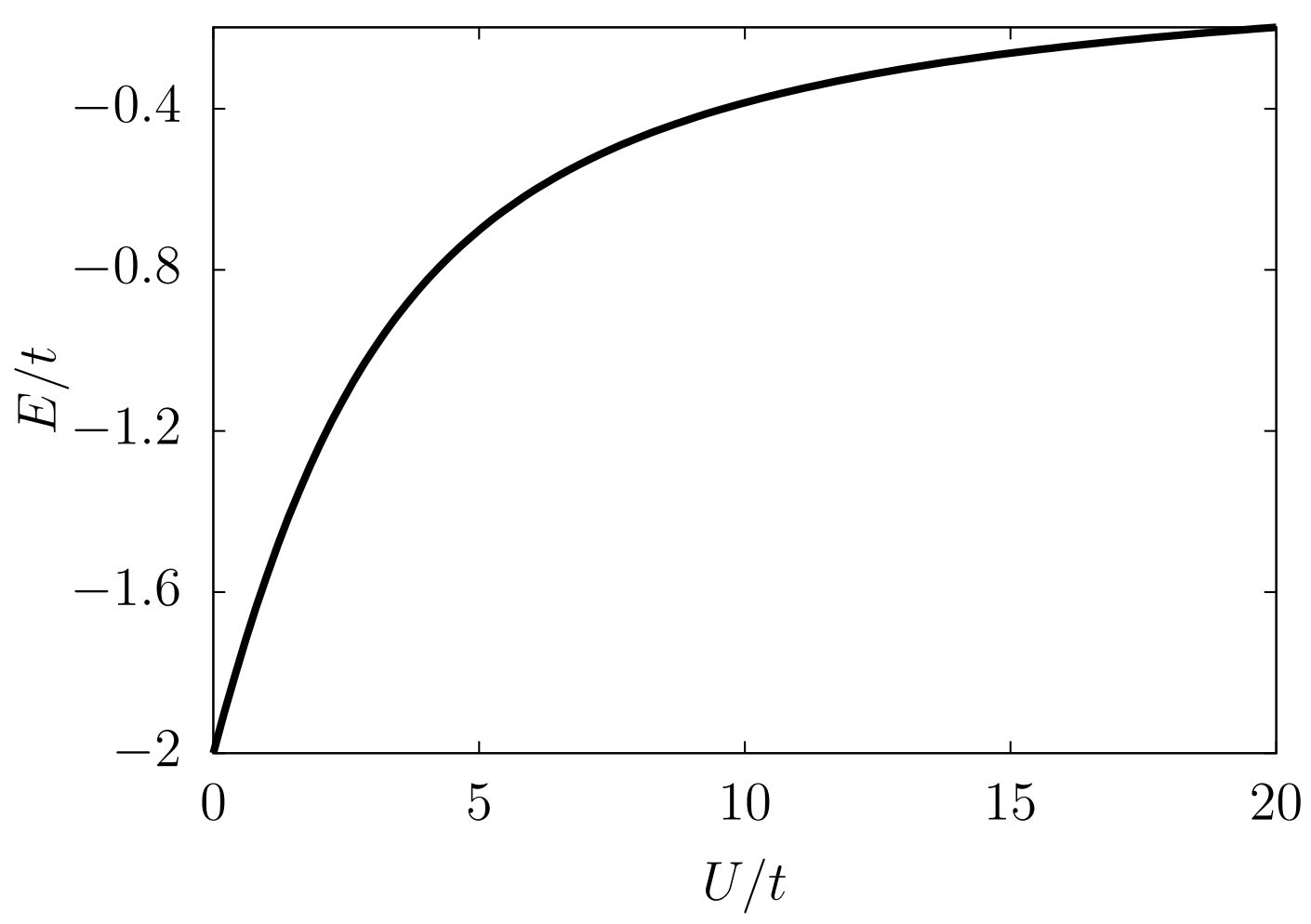

Figure 2.3: The exact ground-state energy $E$ from Eq. 2.126) of the half-filled Hubbard dimer as function of interaction strength $U / t$.

We follow the convention commonly adopted in the solid state community of showing the results for varying interaction strength $U$ and fixed hopping $t$, so that 
the hopping sets the energy scale. Considering the bond dissociation problem, the natural choice would be to keep the interaction strength constant, while reducing the hopping parameter.

For the non-interacting case, i.e. at $U=0$, the wave function is a Slater determinant of bonding states with opposite spin

$$
|\Phi(U=0)\rangle=\frac{1}{2}\left(\hat{c}_{1, \uparrow}^{\dagger}+\hat{c}_{2, \uparrow}^{\dagger}\right)\left(\hat{c}_{1, \downarrow}^{\dagger}+\hat{c}_{2, \downarrow}^{\dagger}\right)|\mathcal{O}\rangle
$$

With $|\mathcal{O}\rangle$ we denote the vacuum state.

This wave function can be rewritten as superposition of two eigenstates of the interaction operator

$$
\begin{aligned}
|\Phi(U=0)\rangle & =\frac{1}{2}\left(\hat{c}_{1, \uparrow}^{\dagger} \hat{c}_{1, \downarrow}^{\dagger}+\hat{c}_{2, \uparrow}^{\dagger} \hat{c}_{2, \downarrow}^{\dagger}\right)|\mathcal{O}\rangle \\
& +\frac{1}{2}\left(\hat{c}_{1, \uparrow}^{\dagger} \hat{c}_{2, \downarrow}^{\dagger}-\hat{c}_{1, \downarrow}^{\dagger} \hat{c}_{2, \uparrow}^{\dagger}\right)|\mathcal{O}\rangle
\end{aligned}
$$

The first wave function contains contributions with two electrons on the same site, i.e. ionic states. Its interaction eigenvalue is $U$. The second wave function describes two electrons with opposite spin on different sites. Its interaction eigenvalue is zero.

The first term describes the double occupancy, that is the probability that two electrons are on the same site, which is penalized by the electron-electron interaction. The second term is attributed to left-right correlation, as it describes the probability that the two electrons are on different sites.

As the interaction strength is increased, the contribution of the first wave function, being responsible for double occupancy, is suppressed. The wave function obtains the form [56]

$$
\begin{array}{rlrl}
|\Phi(\vartheta)\rangle & = & \frac{1}{\sqrt{2}}\left(\hat{c}_{1, \uparrow}^{\dagger} \hat{c}_{1, \downarrow}^{\dagger}+\hat{c}_{2, \uparrow}^{\dagger} \hat{c}_{2, \downarrow}^{\dagger}\right)|\mathcal{O}\rangle \cos \left(\vartheta+\frac{\pi}{4}\right) \\
+ & \frac{1}{\sqrt{2}}\left(\hat{c}_{1, \uparrow}^{\dagger} \hat{c}_{2, \downarrow}^{\dagger}-\hat{c}_{1, \downarrow}^{\dagger} \hat{c}_{2, \uparrow}^{\dagger}\right)|\mathcal{O}\rangle \sin \left(\vartheta+\frac{\pi}{4}\right) .
\end{array}
$$

With a basis set in the order $\left(\left|\chi_{1, \uparrow}\right\rangle,\left|\chi_{1, \downarrow}\right\rangle,\left|\chi_{2, \uparrow}\right\rangle,\left|\chi_{2, \downarrow}\right\rangle\right)$, the one-particle reduced density matrix has the form [56]

$$
\rho_{\alpha, \beta}(\vartheta)=\frac{1}{2}\left(\begin{array}{cccc}
1 & 0 & \cos (2 \vartheta) & 0 \\
0 & 1 & 0 & \cos (2 \vartheta) \\
\cos (2 \vartheta) & 0 & 1 & 0 \\
0 & \cos (2 \vartheta) & 0 & 1
\end{array}\right)
$$


The interaction energy is proportional to the double occupancy

$$
\langle\Phi(\vartheta)|\hat{W}| \Phi(\vartheta)\rangle=U \cos ^{2}\left(\vartheta+\frac{\pi}{4}\right)
$$

and the non-interacting energy is

$$
\langle\Phi(\vartheta)|\hat{h}| \Phi(\vartheta)\rangle=-2 t \cos (2 \vartheta) .
$$

The value of $\vartheta$ results from an equilibrium between the forces from the interaction energy Eq. 2.123) and those from the one-particle energy Eq. 2.124, which determines $\vartheta(U)$ as [56]

$$
\vartheta(U)=\arctan \left(\sqrt{1+\left(\frac{U}{4 t}\right)^{2}}+\frac{U}{4 t}\right)-\frac{\pi}{4} .
$$

The value $\vartheta(U)$ varies from zero to $\pi / 4$ with increasing interaction strength.

The resulting optimum energy has the form

$$
E=-2 t\left[\sqrt{1+\left(\frac{U}{4 t}\right)^{2}}-\frac{U}{4 t}\right]
$$

As the interaction increases, the wave function changes continuously from a Slater determinant of bonding states Eq. (2.120) at $U=0$ to a singlet state with antiferromagnetic correlations [56]. During this process, the bond strength is weakened and the covalent bond vanishes completely in the limit of infinite interaction. This loss of covalent bonding can also be described as localization of electrons on opposite sites, which raises the kinetic energy as a consequence of Heisenberg's uncertainty principle.

What has been described here is what is called static correlation [28]: The states for finite interaction can no more be described by a single Slater determinant, but four Slater determinants are required.

\subsubsection{Natural orbitals and occupations}

Interestingly, the natural orbitals do not depend on the interaction strength $U$. They are the bonding and antibonding states

$$
\begin{aligned}
|b, \sigma\rangle & :=\frac{1}{\sqrt{2}}\left(\left|\chi_{1, \sigma}\right\rangle+\left|\chi_{2, \sigma}\right\rangle\right) \\
|a, \sigma\rangle & :=\frac{1}{\sqrt{2}}\left(\left|\chi_{1, \sigma}\right\rangle-\left|\chi_{2, \sigma}\right\rangle\right) .
\end{aligned}
$$




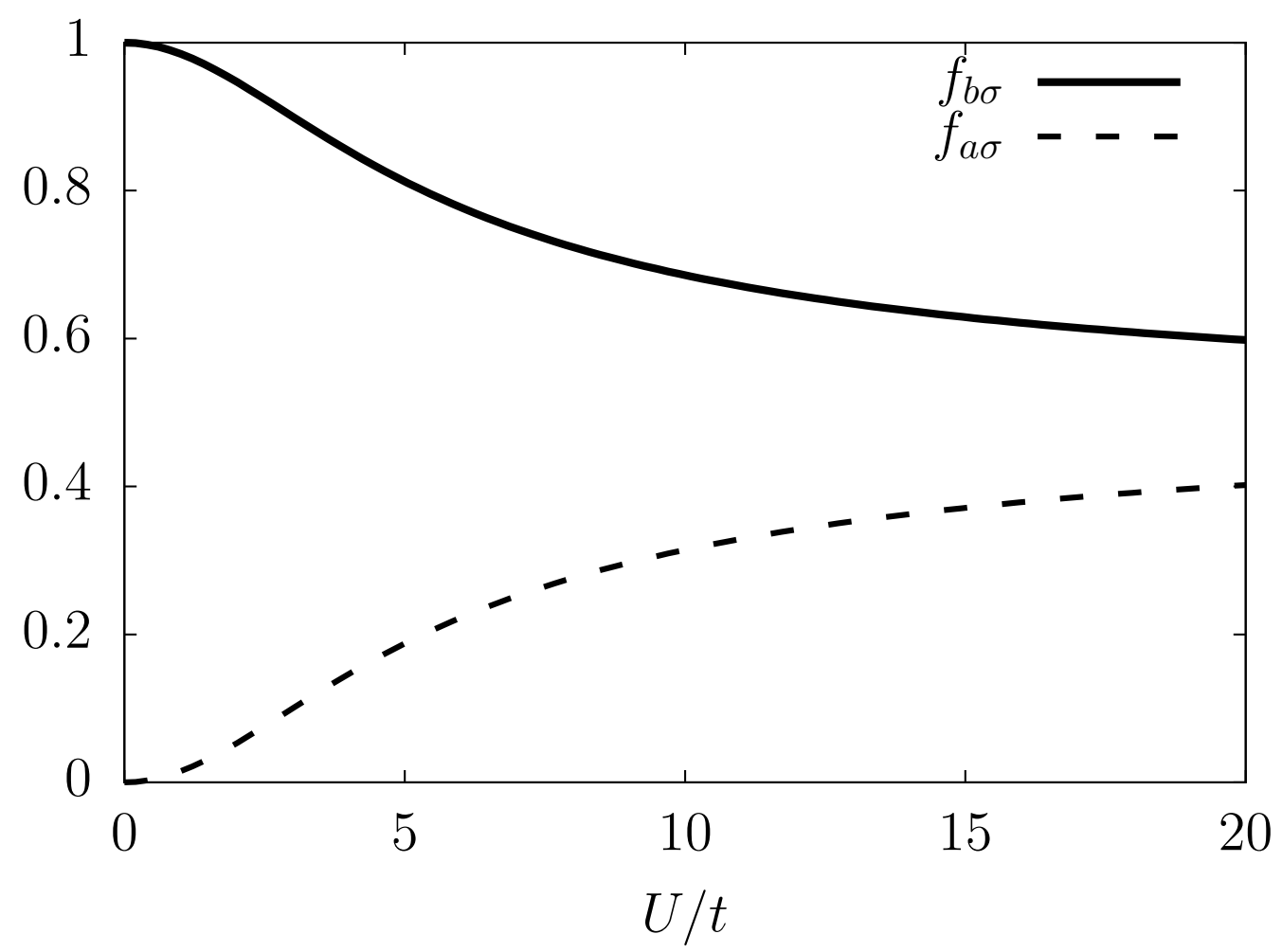

Figure 2.4: The occupations $f_{b, \sigma}$ and $f_{a, \sigma}$ from Eq. 2.128) of the half-filled Hubbard dimer as function of interaction strength $U / t$

Both orbitals are spread over both atoms, and the natural orbitals are identical to those of the non-interacting system.

The loss of bonding is, however, expressed by the fact that the occupations become fractional. The occupations are shown in Fig. 2.4. Their exact values $f_{b, \sigma}$ for the bonding states and $f_{a, \sigma}$ for the antibonding states are [56]

$$
\begin{aligned}
& f_{b, \sigma}=\frac{1}{2}+\frac{1}{2} \cos (2 \vartheta) \\
& f_{a, \sigma}=\frac{1}{2}-\frac{1}{2} \cos (2 \vartheta) .
\end{aligned}
$$

In the non-interacting case, the occupations are integer, with filled bonding states and unoccupied antibonding states. In the limit of large interaction strength the occupations approach $\frac{1}{2}$ for all four natural orbitals. In this limiting case with equally occupied bonding and antibonding states, the net bond strength vanishes completely. In the context of natural orbitals, we describe the effect as quantum fluctuations that create electron-hole pairs. These electron-hole pairs destroy the covalent bond with increasing interaction. [56] 


\subsubsection{Correlations}

In view of the following discussion, it is instructive to investigate the correlations of the electrons. The probability for an electron to be on one site and the other on the other site, we name it "site correlation", is given by the expectation value of

$$
\hat{C}=\left(\hat{c}_{2, \uparrow}^{\dagger} \hat{c}_{2, \uparrow}-\hat{c}_{1, \uparrow}^{\dagger} \hat{c}_{1, \uparrow}\right)\left(\hat{c}_{2, \downarrow}^{\dagger} \hat{c}_{2, \downarrow}-\hat{c}_{1, \downarrow}^{\dagger} \hat{c}_{1, \downarrow}\right) \text {. }
$$

For a state where both electrons bunch on one site, the expectation value of this

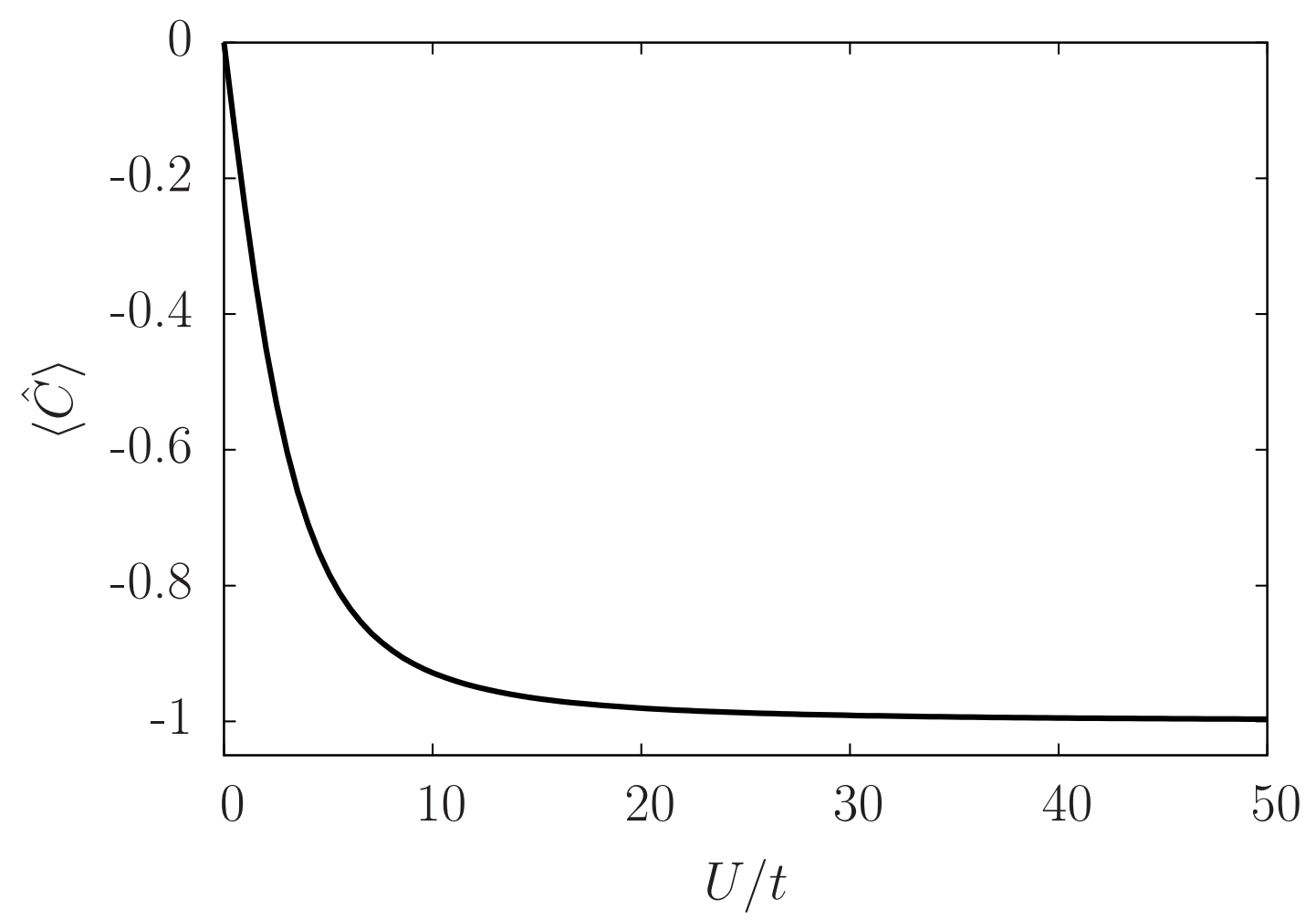

Figure 2.5: Site correlation $\langle\hat{C}\rangle$ as defined in Eq. (2.129) of the half-filled Hubbard dimer as a function of $U / t$. With increasing interaction strength $U / t$ the site correlation shows the transition from delocalized electrons $\langle\hat{C}\rangle=0$ to the left-right correlated state with $\langle\hat{C}\rangle=-1$.

operator is one, while if they localize on opposite sites, the expectation value is minus one. If it is zero, then the electrons are delocalized, i.e there is no correlation between the positions of both electrons. The correlation operator $\hat{C}$ is a two-particle operator and is not accessible from the one-particle density matrix. The exact solution for 
the correlation expectation value for the ground state is given by [56]

$$
\langle\hat{C}\rangle=-\sin (2 \vartheta(U))
$$

where $\vartheta(U)$ is given by Eq. 2.125. We can see in Fig. 2.5 that the site correlation vanishes without interaction, while the electrons anti-bunch for strong correlation so that $\langle\hat{C}\rangle$ approaches minus one. A site correlation of minus one indicates that each electron is fully localized either at one or at the other site, while the other is always at the other site. This is the basic notion of left-right correlation.

Of interest will also be the magnetic nature of the wave functions. The operator for the spin on site $i$ is

$$
\hat{\vec{S}}_{i}=\frac{1}{2}\left(\begin{array}{c}
\hat{c}_{i, \uparrow}^{\dagger} \hat{c}_{i, \downarrow}+\hat{c}_{i, \downarrow}^{\dagger} \hat{c}_{i, \uparrow} \\
-i \hat{c}_{i, \uparrow}^{\dagger} \hat{c}_{i, \downarrow}+i \hat{c}_{i, \downarrow}^{\dagger} \hat{c}_{i, \uparrow} \\
\hat{c}_{i, \uparrow}^{\dagger} \hat{c}_{i, \uparrow}-\hat{c}_{i, \downarrow}^{\dagger} \hat{c}_{i, \downarrow}
\end{array}\right) .
$$

For the wave function in Eq. 2.121), the total spin expectation value $\left\langle\left(\hat{\vec{S}}_{1}+\hat{\vec{S}}_{2}\right)^{2}\right\rangle$ vanishes, and consequently the spin expectation value $\left\langle\hat{\vec{S}}_{i}\right\rangle$ on each site vanishes as well. Nevertheless, the spins on different sites are antiferromagnetically correlated, that is 56 .

$$
\left\langle\Phi(\vartheta)\left|\hat{\vec{S}}_{1} \cdot \hat{\vec{S}}_{2}\right| \Phi(\vartheta)\right\rangle=-\frac{3}{8}[1+\sin (2 \vartheta)] \leq 0
$$

An antiferromagnetic correlation is already present in the non-interacting state, which expresses the non-vanishing contribution of the left-right correlated states to the Slater determinant built from bonding orbitals. As the interaction increases the left-right correlation doubles, which reflects in the increase of the antiferromagnetic correlation expressed in Eq. (2.132). [56] 


\section{Numerical optimization scheme}

In this chapter, we will discuss the numerical optimization scheme used to obtain the grand canonical potential. Throughout this chapter we assume that the density matrix functional is available from one of the methods discussed in Chapter. 2. The problem still persist in the evaluation of grand-canonical potential, which requires to solve a complicated constrained optimization problem of Eq. (2.76). It involves a search for the minima in the space of N-representable one-particle reduced density matrices. We employ the Car-Parinello optimization scheme [57] to tackle the problem of constrained minimization.

\subsection{Historical Background}

Until 1985, the ab-initio molecular-dynamics simulations for large solid state system require computationally demanding calculations for the Born-Oppenheimer surface $E_{g}^{\mathrm{BO}}(\vec{R})$ (See Sec. 2.2). The state-of-the-art density functional methods provide in most cases rather accurate description of the electronic ground state. However these calculations for a fixed position of nuclei, are computationally extremely demanding. Therefore ab-initio molecular dynamics was considered to be a distant dream until 1985.

In the conventional formulation of density functional theory [2, 3], for an approximate density functional one needs to solve a set of Kohn-Sham equations selfconsistently. The solution of Kohn-Sham equations involves repeated matrix diagonalization which scales exponentially with the system size. This makes it practically impossible to study the dynamics of the nuclei, since the whole procedure of iterative diagonalization has to be performed for every nuclei configuration in subsequent time steps.

In 1985, Robert Car and Michelle Parinello [57] radically transformed this situation. They proposed a method where electronic structure calculation were carried out for moving nuclei and the dynamics of nuclei is governed by classical equation of motion.

They approached the problem of calculation of $E_{g}^{\mathrm{BO}}(\vec{R})$ as a complex optimization problem which can be solved by using the concept of simulated annealing introduced by Kirkpatrick et.al [87]. This involves an objective function $O(\beta)$ which is minimized with respect to parameters $\{\beta\}$, by generating a succession of parameters $\{\beta\}^{\prime} s$ with a Boltzmann type probability distribution $\exp (-O(\{\beta\}) / T)$ via a 
Monte-Carlo procedure.

In Car Parinello approach the total energy functional $E\left[\sum_{n}\left|\phi_{n}\right\rangle f\left(x_{n}\right)\left\langle\phi_{n}\right| ; \vec{R}\right]$ is used as an objective function with natural orbitals $\left|\phi_{n}\right\rangle^{\prime} s$, the occupations $f_{n}^{\prime} s$ or $x_{n}^{\prime} s$ and the set of nuclei position vectors $\{\vec{R}\}$ as the variational parameters. The simulated annealing was based on molecular dynamics rather than on Monte Carlo method of Kirkpatrick et.al [87]. They called this approach as dynamic simulated annealing. The variational parameters are considered to be time-dependent and the dynamics of variational parameters are governed by a fictitious Lagrangian

$$
\begin{aligned}
\mathcal{L} & =\frac{1}{2} \sum_{n} m_{f}{\dot{x_{n}}}^{2}+\sum_{n} f\left(x_{n}\right)\left\langle\dot{\phi}_{n}\left|m_{\phi}\right| \dot{\phi_{n}}\right\rangle+\frac{1}{2} \sum_{i=1}^{M} M_{i} \dot{\vec{R}}_{i}^{2} \\
& -E\left[\sum_{n}\left|\phi_{n}\right\rangle f\left(x_{n}\right)\left\langle\phi_{n}\right| ; \vec{R}\right]+\sum_{m n} \Lambda_{m n}\left(\left\langle\phi_{m} \mid \phi_{n}\right\rangle-\delta_{m n}\right)+\mu\left(\sum_{n} f\left(x_{n}\right)-N\right)
\end{aligned}
$$

The dynamics associated with the nuclei in Eq. 3.1 has a real physical meaning whereas the dynamics associated with the occupations and natural orbitals are fictitious and introduced to perform the dynamic simulated annealing. The fictitious mass $m_{f}$ and $m_{\phi}$ should ideally be zero but are chosen to be finite to help in reaching the convergence of the simulated annealing. The occupations, wave-functions and the nuclear coordinates are evolved using Euler-Lagrange equations of motion in the presence of an additional friction.

To obtain convergence to a minima, velocities associated with the parameters can be varied so that the kinetic energy part of the fictitious Lagrangian in Eq. (3.1) decreases. This eventually reduces the associated temperature towards zero and the minima of energy functional is reached.

The major advantage of this proposed scheme by Car and Parinello [57, over the conventional formulation of ab-initio molecular dynamics scheme is that the diagonalization, self-consistency and the atomic relaxations, are all achieved simultaneously.

Details about the material presented in this chapter can be found in the second chapter of lecture notes from P. E. Blöchl [65].

\subsection{Numerical simulation}

Car-Parinello simulations being introduced as an alternative to conventional molecular dynamics simulations can be used essentially for any constrained optimization problem. We will use it extensively for evaluation of the ground state or the grand canonical potential of an electronic system (See Eq. (2.76) ), which in itself is a complex minimization problem. One simplification we have in our work, is that the atom 
positions are fixed. Therefore in the following work, we restrict ourself to a fixed configuration of the nuclei and implement Car-Parinello like simulation for finding the ground state of the electronic system. The nuclear position $\vec{R}$ is no longer a dynamic variable in our calculation, and it is only used as a parameter which for the subsequent discussion will be neglected.

The only dynamic variables of interest are the occupations $f\left(x_{n}\right)$ and the natural orbitals $\left|\phi_{n}\right\rangle$. The equation of motion for the occupations or the free variable $x_{n}$ and the wavefunctions are written in a more suitable form as,

$$
\begin{aligned}
m_{f} \ddot{x}_{n} & =-\frac{\partial E}{\partial x_{n}}+\left(\left\langle\dot{\phi}_{n}\left|m_{\phi}\right| \dot{\phi}_{n}\right\rangle+\mu\right) \frac{d f}{d x_{n}} \\
f\left(x_{n}\right) m_{\phi}\left|\ddot{\phi}_{n}\right\rangle & =-m_{\phi}\left|\dot{\phi}_{n}\right\rangle \dot{f}\left(x_{n}\right)-\frac{\partial E}{\partial\left\langle\phi_{n}\right|}+\sum_{m} \Lambda_{m n}\left|\phi_{m}\right\rangle
\end{aligned}
$$

where partial derivative of the energy functional with respect to $x_{n}$ and $\left|\phi_{n}\right\rangle$ in Eq. 3.2 can be written in a more convenient form as,

$$
\begin{aligned}
\frac{\partial E}{\partial x_{n}} & =\frac{d f}{d x_{n}}\left\langle\phi_{n}|\boldsymbol{D}| \phi_{n}\right\rangle \\
\frac{\partial E}{\partial\left\langle\phi_{n}\right|} & =f_{n} \boldsymbol{D}\left|\phi_{n}\right\rangle \\
\boldsymbol{D} & =\frac{\partial E}{\partial \boldsymbol{\rho}}
\end{aligned}
$$

where $\boldsymbol{D}$ is the derivative matrix of the energy functional $E[\boldsymbol{\rho} ; \vec{R}]$ with respect to one particle reduced density matrix $\boldsymbol{\rho}$ and the elements of the derivative matrix are given by $D_{\alpha \beta}=\frac{\partial E}{\partial \rho_{\beta \alpha}}$. The derivative matrix $\boldsymbol{D}$ and the natural orbitals $\left|\phi_{n}\right\rangle$ can be written in a matrix and column vector form respectively in a convenient basis set $\left\{\left|\chi_{\alpha}\right\rangle\right\}$ which are usually the set of local orbitals. The density matrix elements are defined in this local orbital set as,

$$
\rho_{\alpha \beta}=\sum_{n}\left\langle\chi_{\alpha} \mid \phi_{n}\right\rangle f_{n}\left\langle\phi_{n} \mid \chi_{\beta}\right\rangle
$$

Generally the energy functional is calculated in terms of occupations and natural orbitals and therefore the derivatives available at hand are with respect to occupations as $\frac{\partial E}{\partial f_{n}}$ and with respect to natural orbitals as $\frac{\partial E}{\partial\left\langle\chi_{\alpha} \mid \phi_{n}\right\rangle}$. However for Car-Parinello simulation one needs to evaluate the derivative matrix $\boldsymbol{D}$ which requires calculation of energy functional with respect to one particle reduced density matrix $\boldsymbol{\rho}$. The tranformation from derivatives with respect to occupations and natural orbitals to derivatives with respect to one particle reduced density matrix is discussed in Appendix A. 
To implement the task of minimizing the energy functional $E\left[\sum_{n}\left|\phi_{n}\right\rangle f\left(x_{n}\right)\left\langle\phi_{n}\right|\right]$ it is essential to evolve the occupations and wave functions in the presence of friction (See. Chapter 2 of [65]). The friction quench the system into the ground state (See Chapter 2 of [65]) and it also reproduce the thermal fluctuations of a constant temperature ensemble.

The EOM for the occupation and wavefunction under the influence of friction has the form,

$$
\begin{aligned}
m_{f} \ddot{x_{n}} & =-\left(\left\langle\phi_{n}|\boldsymbol{D}| \phi_{n}\right\rangle-\left\langle\dot{\phi}_{n}\left|m_{\phi}\right| \dot{\phi}_{n}\right\rangle-\mu\right) \frac{d f}{d x_{n}}-m_{f} a_{f} \dot{x}_{n} \\
m_{\phi}\left|\ddot{\phi_{n}}\right\rangle & =-\boldsymbol{D}\left|\phi_{n}\right\rangle+\frac{1}{f\left(x_{n}\right)} \sum_{m} \Lambda_{m n}\left|\phi_{m}\right\rangle-m_{\phi} a_{\phi}\left|\dot{\phi}_{n}\right\rangle
\end{aligned}
$$

where $a_{f}$ and $a_{\phi}$ are the friction coefficients for occupations and wavefunctions repsectively. If we compare the above set of equations with Eq. 3.2, we notice the sign change in the $\left|\dot{\phi}_{n}\right\rangle$ dependent term in EOM for the occupation and the absence of $\left|\dot{\phi}_{n}\right\rangle$ term in the corresponding EOM for the natural orbitals. These set of equations, in the absense of friction term $a_{f}$ and $a_{\phi}$, conserve the total energy during the course of the simulation. The total energy has the form,

$$
\begin{aligned}
E & =\frac{1}{2} \sum_{n} m_{f}{\dot{x_{n}}}^{2}+\sum_{n} f\left(x_{n}\right)\left\langle\dot{\phi}_{n}\left|m_{\phi}\right| \dot{\phi}_{n}\right\rangle+E\left[\sum_{n}\left|\phi_{n}\right\rangle f\left(x_{n}\right)\left\langle\phi_{n}\right|\right] \\
& -\sum_{m n} \Lambda_{m n}\left(\left\langle\phi_{m} \mid \phi_{n}\right\rangle-\delta_{m n}\right)-\mu\left(\sum_{n} f\left(x_{n}\right)-N\right)
\end{aligned}
$$

Under the assumption that the Lagrange multiplier $\boldsymbol{\Lambda}$ is hermitean it can be shown that the energy is conserved for all times i.e. $\frac{d E}{d t}=0$ by using the set of equations in Eq. 3.2 .

\subsubsection{Time evolution}

The equations of motion for the wavefunctions and the occupations in Eq. 3.5 are integrated numerically using Verlet algorithm [88]. The wavefunctions and occupation evolve under constraint which are enforced using Lagrange multipliers $\boldsymbol{\Lambda}$ and the chemical potential $\mu$ respectively. In successive time steps the Lagrange multipliers are evaluated from the constraint conditions.

In order to the solve the EOM numerically, we discretize the time variable as $t_{n}=n \Delta$, where $\Delta$ is the time step. At any time step we need the value of variable 
at three time slices as,

$$
\begin{aligned}
x\left(t_{n}-\Delta\right) & =x(-) \\
x\left(t_{n}\right) & =x(0) \\
x\left(t_{n}+\Delta\right) & =x(+)
\end{aligned}
$$

The first and second order time derivatives in the Verlet algorithm [88] are approximated as,

$$
\begin{aligned}
& \dot{x} \simeq \frac{x(+)-x(-)}{2 \Delta} \\
& \ddot{x} \simeq \frac{x(+)-2 x(0)+x(-)}{\Delta^{2}}
\end{aligned}
$$

Using the approximation for the time derivatives in Eq. 3.8, the discretized version of EOM for the wavefunctions in Eq. 3.5 has the form [65],

$$
\begin{aligned}
m_{\phi} \frac{\left|\phi_{n}(+)\right\rangle-2\left|\phi_{n}(0)\right\rangle+\left|\phi_{n}(-)\right\rangle}{\Delta^{2}} & =-\boldsymbol{D}(0)\left|\phi_{n}(0)\right\rangle-m_{\phi} a_{\phi} \frac{\left|\phi_{n}(+)\right\rangle-\left|\phi_{n}(-)\right\rangle}{2 \Delta} \\
& +\frac{1}{f_{n}} \sum_{m} \Lambda_{m n}\left|\phi_{m}(0)\right\rangle \\
\left|\phi_{n}(+)\right\rangle & =\frac{2}{1+\alpha_{\phi}}\left|\phi_{n}(0)\right\rangle-\frac{1-\alpha_{\phi}}{1+\alpha_{\phi}}\left|\phi_{n}(-)\right\rangle \\
& -\frac{\Delta^{2}}{m_{\phi}\left(1+\alpha_{\phi}\right)} \boldsymbol{D}(0)\left|\phi_{n}(0)\right\rangle \\
& +\frac{1}{f_{n}} \sum_{m} \Lambda_{m n} \frac{\Delta^{2}}{m_{\phi}\left(1+\alpha_{\phi}\right)}\left|\phi_{m}(0)\right\rangle
\end{aligned}
$$

where $\alpha_{\phi}=\frac{a_{\phi} \Delta}{2}$. The wavefunction at time $t_{n}+\Delta$ is evaluated in terms of wavefunction at time slice $t_{n}$ and $t_{n}-\Delta$ and the Lagrange multipliers $\Lambda$ which has to be determined independently is discussed later in this section.

The discretized version of the EOM for occupation $f_{n}$ or the free variable $x_{n}$ which parametrizes the occupation has the form [65],

$$
\begin{aligned}
m_{f} \frac{x_{n}(+)-2 x_{n}(0)+x_{n}(-)}{\Delta^{2}} & =-\left\langle\phi_{n}(0)|\boldsymbol{D}| \phi_{n}(0)\right\rangle-m_{f} a_{f} \frac{x_{n}(+)-x_{n}(-)}{2 \Delta} \\
& +\mu \frac{d f_{n}}{d x_{n}(0)} \\
x_{n}(+) & =\frac{2}{1+\alpha_{f}} x_{n}(0)-\frac{1-\alpha_{f}}{1+\alpha_{f}} x_{n}(-)
\end{aligned}
$$




$$
-\frac{\Delta^{2}}{m_{f}\left(1+\alpha_{f}\right)}\left\langle\phi_{n}(0)|\boldsymbol{D}| \phi_{n}(0)\right\rangle+\frac{\Delta^{2}}{m_{f}\left(1+\alpha_{f}\right)} \mu \frac{d f_{n}}{d x_{n}(0)}
$$

where $\alpha_{f}=\frac{a_{f} \Delta}{2}$ and the lagrange multiplier $\mu$ is determined independently to enforce the number of particles constraint.

\subsection{Constraints}

\subsubsection{Wave function constraint}

As stated in the previous section, the dynamics of wavefunctions and occupations in Eq. 3.5 require the evaluation of Lagrange multiplieres $\Lambda$ and $\mu$ at each time step $t_{n}$. The constraint condition for wavefunctions to be orthonormal at each time step i.e $\left\langle\phi_{n}(+) \mid \phi_{m}(+)\right\rangle=\delta_{n m}$, determines the Lagrange multiplier $\boldsymbol{\Lambda}$. The wavefunction $\left|\phi_{n}(+)\right\rangle$ is given by Eq. 3.9 and has the form,

$$
\begin{aligned}
\left|\phi_{n}(+)\right\rangle & =\left|\tilde{\phi}_{n}\right\rangle+\sum_{m} \frac{1}{f\left(x_{n}\right)} \Lambda_{m n}\left|\psi_{m}\right\rangle \\
\left|\tilde{\phi}_{n}\right\rangle & =\frac{2}{1+\alpha_{\phi}}\left|\phi_{n}(0)\right\rangle-\frac{1-\alpha_{\phi}}{1+\alpha_{\phi}}\left|\phi_{n}(-)\right\rangle-\frac{\Delta^{2}}{m_{\phi}\left(1+\alpha_{\phi}\right)} \boldsymbol{D}(0)\left|\phi_{n}(0)\right\rangle \\
\left|\psi_{m}\right\rangle & =\frac{\Delta^{2}}{m_{\phi}\left(1+\alpha_{\phi}\right)}\left|\phi_{m}(0)\right\rangle
\end{aligned}
$$

Using the fact that the wavefunctions in the current $\left|\phi_{n}(0)\right\rangle$ and previous $\left|\phi_{n}(-)\right\rangle$ time steps have been orthonormalized, the constraint condition $\left\langle\phi_{n}(+) \mid \phi_{m}(+)\right\rangle=$ $\delta_{n m}$ leads to,

$$
\begin{aligned}
\left(\left\langle\tilde{\phi}_{n} \mid \tilde{\phi}_{m}\right\rangle-\delta_{m n}\right)+ & \sum_{i}\left\langle\tilde{\phi}_{n} \mid \psi_{i}\right\rangle \frac{1}{f\left(x_{m}\right)} \Lambda_{i m}+\sum_{i} \frac{1}{f\left(x_{n}\right)} \Lambda_{i n}^{*}\left\langle\psi_{i} \mid \tilde{\phi}_{m}\right\rangle \\
+ & \sum_{i j} \frac{1}{f\left(x_{n}\right)} \Lambda_{i n}^{*}\left\langle\psi_{i} \mid \psi_{j}\right\rangle \frac{1}{f\left(x_{m}\right)} \Lambda_{j m}=0 \\
& \boldsymbol{A}+\boldsymbol{B} \boldsymbol{X}+\boldsymbol{X}^{\dagger} \boldsymbol{B}^{\dagger}+\boldsymbol{X}^{\dagger} \boldsymbol{C} \boldsymbol{X}=0
\end{aligned}
$$

where the matrices $\boldsymbol{A}_{i j}=\left\langle\tilde{\phi}_{i} \mid \tilde{\phi}_{j}\right\rangle-\delta_{i j}, \boldsymbol{B}_{i j}=\left\langle\tilde{\phi}_{i} \mid \psi_{j}\right\rangle$ and $\boldsymbol{X}_{i j}=\frac{1}{f\left(x_{j}\right)} \Lambda_{i j}$. The fact that $\boldsymbol{X}$ is hermitean, the matrix quadratic equation can be solved using an iterative procedure (For more details See Chapter 2 of [65]). 


\subsubsection{Occupation constraint}

The number of electrons $N$ in the system during the course of dynamics are enforced using the Lagrange multiplier $\mu$. Thus for all successive time steps the occupations should add up to the number of electrons $N$ for a given value of $\mu$ as

$$
\sum_{n} f\left(x_{n}(+)\right)=N(\mu)
$$

The optimum value of $\mu$ which enforces the constant filling can be evaluated from Eq. 3.13 via a root search algorithm like bisection or Newton-Raphson method. We use Newton-Raphson algorithm for root finding in our existing code.

\subsection{Convergence, stability and accuracy}

The stability and accuracy of Car-Parinello simulations depend on the accuracy of the numerical integrator used to integrate the equations of motion in Eq.(3.5). We use Verlet algorithm as an integrator which is very simple and robust but not very accurate.

The general form of equations in the Car-Parinello simulations barring the constraint terms is that of a damped harmonic osscilator which will be used to analyze the accuracy and stability of the Verlet algorithm. The damped harmonic oscillator will also be used as a prototype system to evaluate the optimium values of the friction and the time step in the Car-Parinello simulations. The optimum time-step is essential for the stability and accuracy of Verlet integrator whereas the friction term is the deciding factor for the convergence of the Car Parinello simulation.

\subsubsection{Accuracy and Stability of Verlet Algorithm}

The prototype used to analyze the stability and convergence issues is the damped harmonic oscillator. The equation of motion has the form,

$$
m \ddot{r}+m \alpha \dot{r}=F(t)
$$

where $F(t)=-k r(t)$. The error accumulated in each time step due to the finite discretization of equation of motion can be understood from the discretized form of $\ddot{r} \simeq \frac{r(+)-2 r(0)+r(-)}{\Delta^{2}}$ and $\dot{r} \simeq \frac{r(+)-r(-)}{2 \Delta}$. The error in this approximation of first and second time derivative can be calculated from the Taylor expansion of $r(+)$ and $r(-)$ as,

$$
\begin{aligned}
& r(+)=r(0)+\dot{r} \Delta+\frac{1}{2} \ddot{r} \Delta^{2}+\ldots \\
& r(-)=r(0)-\dot{r} \Delta+\frac{1}{2} \ddot{r} \Delta^{2}+\ldots
\end{aligned}
$$


The error in velocity and accelaration due to finite time discretization goes as,

$$
\begin{aligned}
\frac{r(+)-r(-)}{2 \Delta} & =\dot{r}+\frac{1}{6} r^{(3)} \Delta^{2}+. . \\
\frac{r(+)-2 r(0)+r(-)}{\Delta^{2}} & =\ddot{r}+\frac{1}{12} r^{(4)} \Delta^{2}+. .
\end{aligned}
$$

The error in velocity and accelaration is $O\left(\Delta^{2}\right)$. The position in subsequent time step of Verlet algorithm is given by,

$$
r(+)=\frac{2}{1+\alpha \Delta / 2} r(0)-\frac{1-\alpha \Delta / 2}{1+\alpha \Delta / 2} r(-)+F(0) \frac{\Delta^{2}}{m} \frac{1}{1+\alpha \Delta / 2}
$$

Thus the evaluation of $r$ in each time step lead to an accumulation of errors which goes as $O\left(\Delta^{4}\right)$ since the force term itself is multiplied by $\Delta^{2}$. The total number of time steps for one simulation are of the order of $1 / \Delta$, thus the total error in the simulation is of the order of $O\left(\Delta^{3}\right)$. Since the errors in each time step depend only on the even powers of $\Delta$, thus the Verlet algorithm preserves the time inversion symmetry. Hence there is no energy drift in the course of evolution and in the absence of friction, Verlet algorithm conserves the total energy.

The stability of the Verlet algorithm depends on the time step $\Delta$ which has the stability limit (For details, See Chapter 2 of [65]),

$$
\Delta<\frac{2}{\omega_{0}}
$$

where $\omega_{0}$ is the maximum frequency of the modes in the system. For eg. in case of a harmonic oscillator $(F(t)=-k r(t))$ the characteristic frequency has the form $\omega_{0}=\sqrt{k / m}$, where $k$ is the spring constant. For a simple harmonic oscillator, the simulated frequency $\omega$ of the system due to finite time-step, is given in terms of characteristic frequency $\omega_{0}$ as,

$$
\frac{\omega}{\omega_{0}}=\frac{1}{\omega_{0} \Delta} \arccos \left(1-\frac{\left(\omega_{0} \Delta\right)^{2}}{2}\right)
$$

For the solution to exist $\omega_{0} \Delta<2$. In the extreme limit of $\omega_{0} \Delta \rightarrow 0$ only, we can recover the exact characteristic frequency of the system from the simulation. At the stability limit of $\omega_{0} \Delta=2$, the frequencies are overestimated by a factor of $\frac{\pi}{2}$. Calculations have been done in great details in Chapter 2 of [65].

\subsubsection{Convergence}

The convergence to the minima depends on the energy dissipation of the system. The behvaior of energy dissipation in the presence of friction can be understood 
analytically in the simple case of damped harmonic oscillator which has the form as in Eq. 3.14. The rate of energy dissipation $\frac{d E}{d t}$ for the damped harmonic oscillator has the form,

$$
\frac{d E}{d t}=-\alpha\left(\frac{d x}{d t}\right)^{2}
$$

It is evident from the Eq. 3.20$)$, that the rate of dissipation or consequentially the convergence rate is directly proportional to the square of velocity and the friction. At the first glance it seems that if the friction is large, one will reach the minima faster, but larger friction leads to smalled velocities and the system cannot accumulate kinetic energy and thus the motion will be extremely slow. In the other extreme case of small friction, its obvious that system will not dissipate the kinetic energy efficiently. Thus we have to find an optimum friction at which the kinetic energy of the system is dissipated efficiently and leads to proper convergence to the minima.

The optimum friction for the simulation of damped harmonic oscillator can be obtained analytically (See Chapter 2 [65]) and it has the following value,

$$
a=\alpha \Delta / 2=\omega_{0} \Delta
$$

It can be shown [65] that due to Verlet discretization, the imaginary part of the simulation frequency that controls the decay of the trajectory, has a maxima at the optimum value of friction given by Eq. (3.21).

In practice the friction is chosen at a particular value, such that the total energy in Eq. (3.6) follows an optimum damped trajectory, similar to that of damped harmonic oscillator. When the energy change in subsequent time steps becomes negligible, the friction is switched off and the system is allowed to evolve from its lastest configuration. If the ground state of the system was reached when the friction was switched off, then the further evolution in the absence of friction will lead to oscillation with neglible amplitude around converged value. This criteria should be used to avoid the risk of getting stuck in a local minima or the meta-stable state. The most convenient way is to allow the friction to reduce in each successive iteration. Also to avoid bias, the natural orbitals and occupations should be chosen randomly and to ensure the global minima, simulations should be performed for different initial random configurations.

In Chapter. 4, with regards to a specific model, we will discuss the criteria which works best, to avoid bias in the simulations and increasing the possibility of landing in a global minima. 



\section{Parametrized density matrix functionals}

This chapter contains results and figures obtained from the publication of E. Kamil, R. Schade, T. Pruschke and P. E. Blöchl [56]. All the authors contributed equally and should be considered as co-first authors. The results in Sec. 4.7 are obtained from the calculations done solely by Robert Schade.

The exact formulation of density matrix functionals via wave-function (See Sec. 2.6.1) and Green's function (See Sec. 2.6.2) based methods has the same complexity as the exact evaluation of grand canonical potential for the many-body system. In order to avoid the full complexity of an explicit many-body description, one proceeds analogously to the development of density functionals, namely by searching models for the density-matrix functional, that capture the most essential physical effects while having an algebraic dependence on the density matrix as discussed in Sec. 2.6.3.

In this chapter we study a class of Hartree-Fock like density matrix functionals namely Müller [50] and Power functionals [53]. The development of such model density matrix functional relies on the benchmark system that allows one to evaluate their quality and for this purpose we use exactly solvable system like the Hubbard dimer as described in Section 2.7.1.

\subsection{Grand canonical potential and the density matrix functional}

As discussed in Chapter 2, the grand canonical potential for finite temperature and fixed particle number, for a many-particle system is expressed with the help of the density-matrix functional $F_{\beta}^{\hat{W}}[\boldsymbol{\rho}]$ as

$$
\begin{aligned}
\Omega_{\beta}^{\hat{h}+\hat{W}}= & \min _{f_{n} \in[0,1],\left|\phi_{n}\right\rangle}\left\{\sum_{n} f_{n}\left\langle\phi_{n}|\hat{h}| \phi_{n}\right\rangle-\mu \sum_{n} f_{n}+F_{\beta}^{\hat{W}}\left[\sum_{n} f_{n}\left|\phi_{n}\right\rangle\left\langle\phi_{n}\right|\right]\right. \\
& \left.-\sum_{m n} \Lambda_{m n}\left(\left\langle\phi_{n} \mid \phi_{m}\right\rangle-\delta_{m n}\right)\right\}
\end{aligned}
$$


where $\hat{h}=\sum_{\alpha, \beta}\left|\chi_{\alpha}\right\rangle h_{\alpha, \beta}\left\langle\chi_{\beta}\right|$.

The reduced density-matrix functional $F_{\beta}^{\hat{W}}[\boldsymbol{\rho}]$ is universal in the sense that it depends only on the intrinsic properties of the electron gas, namely the interaction $\hat{W}$, while it is independent of the one-particle Hamiltonian $\hat{h}$. The chemical potential $\mu$ is a Lagrange multiplier that constrains the electron number to $N . \Lambda_{m n}$ are the Lagrange multipliers which enforce that natural orbitals $\left|\phi_{m}\right\rangle$ remain orthonormal.

It is customary to separate the Hartree contribution from the density matrix functional as,

$$
F_{\beta}^{\hat{W}}[\boldsymbol{\rho}]=E_{H}[\boldsymbol{\rho}]+U_{x c, \beta}[\boldsymbol{\rho}]
$$

where the exchange-correlation energy $U_{x c}$ contains the complexity of the many particle problem. It is the electrostatic interaction of each electron with its exchangecorrelation hole and the entropy term $-T S$.

The Hartree energy $E_{H}[\boldsymbol{\rho}]$ is obtained from the electron density $n(\vec{r})$ defined in Eq. (2.32) as,

$$
\begin{aligned}
n(\vec{r}) & =\sum_{\sigma} \rho(r \sigma, r \sigma) \\
& =\sum_{\sigma} \sum_{\alpha, \beta} \chi_{\alpha}(\vec{r}, \sigma) \rho_{\alpha, \beta} \chi_{\beta}^{*}(\vec{r}, \sigma)
\end{aligned}
$$

as

$$
\begin{aligned}
E_{H}[\boldsymbol{\rho}] & =\frac{1}{2} \int d^{3} r \int d^{3} r^{\prime} \frac{e^{2} n(\vec{r}) n\left(\overrightarrow{r^{\prime}}\right)}{4 \pi \epsilon_{0}\left|\vec{r}-\overrightarrow{r^{\prime}}\right|} \\
& =\frac{1}{2} \sum_{\alpha, \beta, \gamma, \delta} W_{\alpha, \beta, \delta, \gamma} \rho_{\delta, \alpha} \rho_{\gamma, \beta} .
\end{aligned}
$$

where the interaction tensor element $W_{\alpha \beta, \gamma \delta}$ is evaluated as in Eq.(2.24)

Towards the main goal of testing the quality of available approximate density matrix functionals, we now proceed towards the discussion of hole function as defined in Eq.2.43), for the construction of approximate exchange-correlation energy $U_{x c}$.

\subsection{Construction of density-matrix functionals}

As discussed in Sec. 2.6.3, most empirical density-matrix functionals maintain the general form of the Hartree-Fock exchange term. However the dependence on the occupations is no longer quadratic, rather it has a general form given by,

$$
U_{x c}[\boldsymbol{\rho}]=-\frac{1}{2} \sum_{m, n} c_{m, n} \sum_{\alpha \beta \gamma \delta} U_{\alpha \beta, \delta \gamma}
$$




\begin{tabular}{|l|l|}
\hline \hline Hartree-Fock approximation & $c_{m, n}^{H F}=f_{m} f_{n}$ \\
Müller functional[50] & $c_{m, n}^{M}=f_{m}^{\frac{1}{2}} f_{n}^{\frac{1}{2}}$ \\
power functional[53] & $c_{m, n}^{P}(\alpha)=f_{m}^{\alpha} f_{n}^{\alpha}$ \\
\hline \hline
\end{tabular}

Table 4.1: Dependence of the parameters $c_{m, n}$ on the occupations $f_{n}$ as defined in Eq. 4.5 for density-matrix functionals used in this work.

$$
\times\left\langle\chi_{\gamma} \mid \phi_{m}\right\rangle\left\langle\phi_{m} \mid \chi_{\alpha}\right\rangle\left\langle\chi_{\delta} \mid \phi_{n}\right\rangle\left\langle\phi_{n} \mid \chi_{\beta}\right\rangle,
$$

where we replace the factor $f_{n} f_{m}$ in Eq. (2.99) by coefficients $c_{m, n}$ with a different dependence on the occupations.

The coefficients $c_{m, n}$ for the approximate density-matrix functionals considered in this work are summarized in table 4.1 .

The power functional therefore interpolates between uncorrelated Hartree-Fock functional $(\alpha=1)$ and the over correlated Müller functional $\left(\alpha=\frac{1}{2}\right)$

\subsection{Optimization scheme for the evaluation of the grand canonical potential}

With the different approximations of exchange-correlation energy $U_{x c}$ in Sec. 4.2 , the density matrix functional in Eq. (4.2) attains a simple algebraic form. Using the different approximations for the density matrix functional, we need to optimize the expression on the right hand side of Eq. 4.1) over the space of natural orbitals $\left|\phi_{n}\right\rangle$ and the occulations $f_{n}$ under certain constraints. We employ the technique of Car-Parinello optimization discussed in Chapter. 3. The fictitious Lagrangian has the form,

$$
\begin{aligned}
\mathcal{L} & =\frac{1}{2} \sum_{n} m_{f} \dot{x}_{n}^{2}+\sum_{n} f\left(x_{n}\right) m_{\psi} \sum_{\alpha}\left|\dot{a}_{\alpha, n}\right|^{2} \\
& -\sum_{n} f\left(x_{n}\right) \sum_{\alpha, \beta} a_{\alpha, n} h_{\beta, \alpha} a_{\beta, n}^{*}-F^{\hat{W}}\left[\sum_{n} a_{\alpha, n} f\left(x_{n}\right) a_{\beta, n}^{*}\right] \\
& +\sum_{n, m} \Lambda_{m, n}\left(\sum_{\alpha} a_{\alpha, n}^{*} a_{\alpha, m}-\delta_{n, m}\right)+\mu\left(\sum_{n} f\left(x_{n}\right)-N\right) .
\end{aligned}
$$


where the constraint of occupation being $0 \leq f_{n} \leq 1$ is usually bypassed using the following parametrization with free variables $x_{n}$ as,

$$
f_{n}=\frac{1}{2}\left(1-\cos x_{n}\right)
$$

The natural orbitals $\left|\phi_{n}\right\rangle$ are represented as a linear combination of local orbitals $\left|\chi_{\alpha}\right\rangle$ as,

$$
\left|\phi_{n}\right\rangle=\sum_{\alpha}\left|\chi_{\alpha}\right\rangle a_{\alpha, n}
$$

The orthonormality of the natural orbitals is enforced with the Lagrange multipliers $\Lambda_{m, n}$, which form a hermitian matrix, and the particle number is constrained with the chemical potential $\mu$.

In order to avoid any bias in our results, the wave functions and occupations are initialized as random numbers between zero and one. Then the constraints, i.e. orthonormality of the natural orbitals and total particle number, are imposed. The Car-Parinello dynamics as discussed in Chapter. 3 is then performed on the parameters $\left\{x_{n}\right\}$ and $\left\{a_{\alpha_{n}}\right\}$.

The phase space is explored by repeating the calculation, in order to identify the global minimum and potential degenerate ground states. To obtain an analytical understanding of the solutions we analyse the form of the natural orbitals by the inspection of the results obtained numerically. The resulting ansatz for the natural orbitals is then verified by optimizing the total energy in this subspace, and comparing the energies.

With the recipe in hand we now discuss the results of E. Kamil, R. Schade, T. Pruschke and P. E. Blöchl [56], in details. They disuss the quality of Hartree-Fock, Müller and power density matrix functionals by benchmarking their results on an exactly solvable system of the Hubbard Dimer (Ref. Sec. 2.7.1). They also performed an indepth analytical study of these approximate density matrix functionals and highlighted the extreme caution to be taken while implementing it for the real systems.

\subsection{Performance of density-matrix functionals}

\subsubsection{Hartree-Fock approximation}

To begin with we study the effect of Hartree-Fock density matrix functional given by Eq. 2.99), on the half filled Hubbard dimer and compare it with exact results from Sec. 2.7.1. 


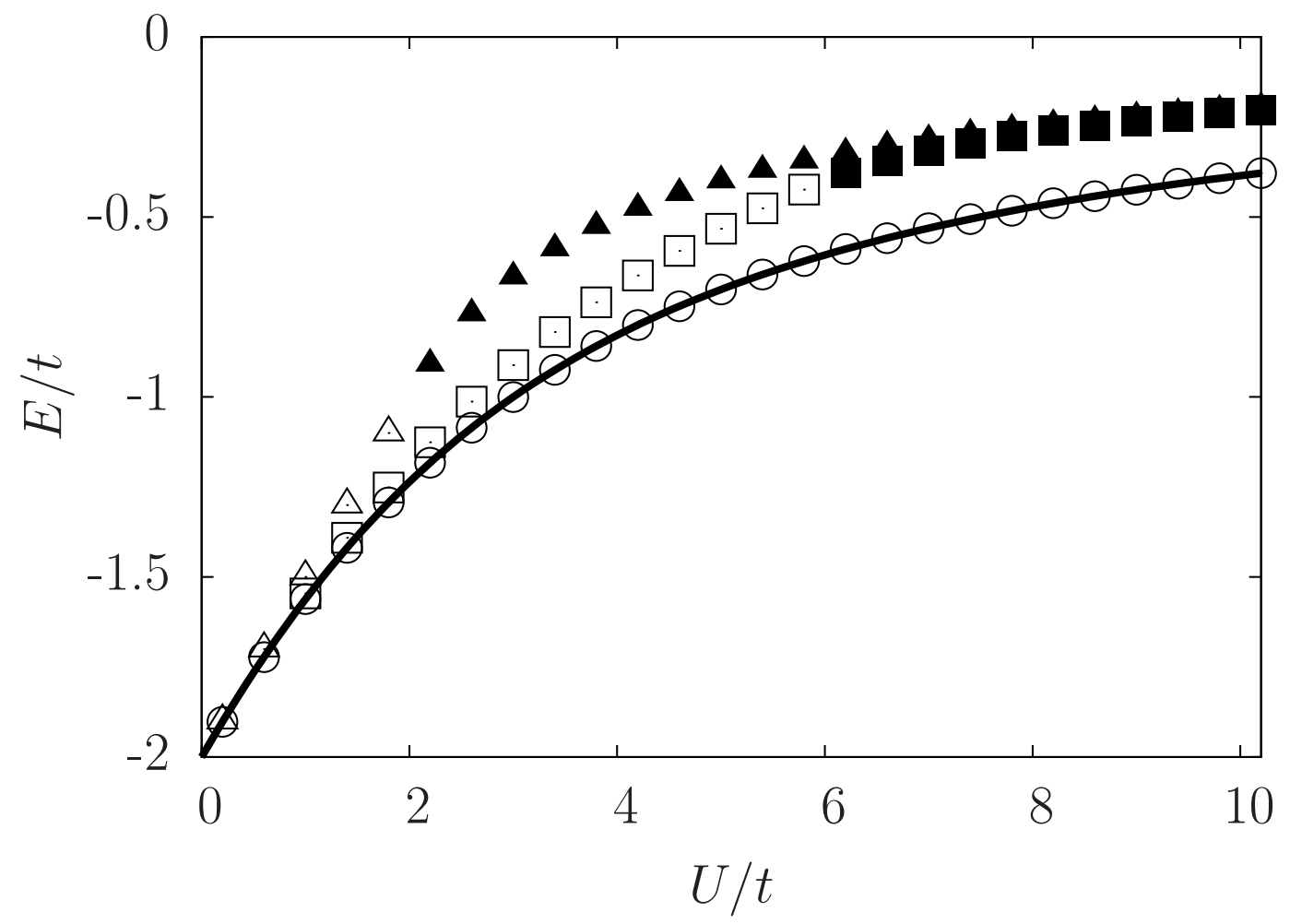

Figure 4.1: Ground-state energy $E$ of the half-filled Hubbard dimer as function of interaction strength $U / t$ for different density-matrix functionals. Circles: Müller functional. Squares: power functional with $\alpha=0.53$. Triangles: Hartree-Fock approximation. Solid line: exact ground-state energy. Müller's functional produces the correct ground-state energy at half filling. Non-magnetic states are indicated by open symbols and antiferromagnetic states by filled symbols.

\section{Non magnetic solution}

If the optimization over one particle reduced density matrices in Eq.(4.1) is constrained over a space of non-magnetic density matrices i.e. the occupations for the up and down spins are same, then the natural orbitals turns out be interaction strength independent. The total energy has the following form,

$$
E^{H F}(U)=-2 t+\frac{U}{2} .
$$

which states that in the dissociation limit of the hydrogen molecule i.e. $t=0$, the total energy is equal to the half of the interaction strength $U$. This however is not true at half-filling in the exact system, as the total energy vanishes, because each isolated atom has single electron that does not interact with each other. This 
reflects the well known difficulty of restricted, i.e. non-spin-polarized, Hartree-Fock to describe the dissociation of chemical bonds.

Today's electronic structure calculations should consider a spin-polarization whenever a magnetization provides a lower energy. Allowing for spin polarization, i.e. as in unrestricted Hartree-Fock or spin-density functional theory, improves the description dramatically. Nevertheless, the transition from the weakly correlated to the strongly correlated regime still differs in many ways from the correct behavior. These differences are of interest in the following discussion.

\section{Anti-ferromagnetic solution}

As evident from Fig. 4.1, if one allows for an unbiased optimization, over a complete space of density matrices, then the Hartree-Fock density matrix functional leads to a transition from non-magnetic to anti-ferromagnetic solutions at a critical value of interaction of $U=2 t$. To get an analytical understanding of this transition, the natural orbitals obtained from the unbiased optimization were analysed and a minimal ansatz for the natural orbitals is proposed.

One such ansatz for the natural orbitals has the form,

$$
\begin{aligned}
\left|\phi_{1}^{H F}(\gamma)\right\rangle & =+|b, \uparrow\rangle \cos (\gamma)+|a, \uparrow\rangle \sin (\gamma) \\
\left|\phi_{2}^{H F}(\gamma)\right\rangle & =+|b, \downarrow\rangle \cos (\gamma)-|a, \downarrow\rangle \sin (\gamma) \\
\left|\phi_{3}^{H F}(\gamma)\right\rangle & =-|b, \uparrow\rangle \sin (\gamma)+|a, \uparrow\rangle \cos (\gamma) \\
\left|\phi_{4}^{H F}(\gamma)\right\rangle & =+|b, \downarrow\rangle \sin (\gamma)+|a, \downarrow\rangle \cos (\gamma) .
\end{aligned}
$$

where $|b, \sigma\rangle$ and $|a, \sigma\rangle$ are the bonding and anti-bonding orbitals respectively defined in terms of local orbitals $\left|\chi_{i, \sigma}\right\rangle$ as,

$$
\begin{aligned}
|b, \sigma\rangle & :=\frac{1}{\sqrt{2}}\left(\left|\chi_{1, \sigma}\right\rangle+\left|\chi_{2, \sigma}\right\rangle\right) \\
|a, \sigma\rangle & :=\frac{1}{\sqrt{2}}\left(\left|\chi_{1, \sigma}\right\rangle-\left|\chi_{2, \sigma}\right\rangle\right) .
\end{aligned}
$$

This is the simplest ansatz for the natural orbitals for the Hartree-Fock solution which can be parametrized by only one free parameter $\gamma$.

The first two natural orbitals are occupied and the last two are the unoccupied. The many particle wave-function for the Hartree-Fock solution is a single Slater determinant which can be constructed from the natural orbitals as,

$\left|\Phi^{H F}(\gamma)\right\rangle=\left[\hat{c}_{1, \uparrow}^{\dagger} \cos \left(\gamma-\frac{\pi}{4}\right)+\hat{c}_{2, \uparrow}^{\dagger} \cos \left(\gamma+\frac{\pi}{4}\right)\right]\left[\hat{c}_{1, \downarrow}^{\dagger} \cos \left(\gamma+\frac{\pi}{4}\right)-\hat{c}_{2, \downarrow}^{\dagger} \cos \left(\gamma-\frac{\pi}{4}\right)\right]|\mathcal{O}\rangle$, 
With the given many particle wave-function $\left|\Phi^{H F}(\gamma)\right\rangle$, we can evaluate the interaction energy as,

$$
\left\langle\Phi^{H F}(\gamma)|\hat{W}| \Phi^{H F}(\gamma)\right\rangle=\frac{1}{2} U \cos ^{2}(2 \gamma)
$$

and the kinetic energy requires the evaluation of the one particle reduced density matrix $\rho^{H F}(\gamma)$ which can be obtained from the many particle wave-function $\left|\Phi^{H F}(\gamma)\right\rangle$ as,

$$
\rho^{H F}(\gamma)_{\alpha \beta}=\left\langle\Phi^{H F}(\gamma)\left|\hat{c}_{\beta}^{\dagger} \hat{c}_{\alpha}\right| \Phi^{H F}(\gamma)\right\rangle
$$

In the local orbitals $\left(\left|\chi_{1, \uparrow}\right\rangle,\left|\chi_{1, \downarrow}\right\rangle,\left|\chi_{2, \uparrow}\right\rangle,\left|\chi_{2, \downarrow}\right\rangle\right)$ basis, the one particle reduced density matrix has the form,

$$
\rho^{H F}(\gamma)=\frac{1}{2}\left(\begin{array}{cccc}
1+\sin (2 \gamma) & 0 & \cos (2 \gamma) & 0 \\
0 & 1-\sin (2 \gamma) & 0 & \cos (2 \gamma) \\
\cos (2 \gamma) & 0 & 1-\sin (2 \gamma) & 0 \\
0 & \cos (2 \gamma) & 0 & 1+\sin (2 \gamma)
\end{array}\right)
$$

Using the one particle Hamiltonian $\hat{h}$ for the Hubbard dimer as given in Eq. 2.115), the kinetic energy has the following form,

$$
\operatorname{Tr}\left(\hat{h} \rho^{H F}(\gamma)\right)=-2 t \cos (2 \gamma)
$$

The minimum condition for the total energy is given by,

$$
\gamma(U)= \begin{cases}0 & \text { for } U \leq 2 t \\ \frac{1}{2} \arccos \left(\frac{2 t}{U}\right) & \text { for } U>2 t\end{cases}
$$

For $U \leq 2 t$, the system remains non-magnetic and the natural orbitals are given by bonding and antibonding orbitals as in the case of non-magnetic dimer. But for $U>2 t$, the system becomes an antiferromagnet, whereas the exact many-particle wave function is a singlet with antiferromagnetic correlations (See Sec.2.7.1).

\subsubsection{Müller's functional}

It is evident from Fig. 4.1, that the ground state energy obtained from unbiased Car-Parinello optmization using the Müller functional i.e. $c_{m n}\left(\alpha=\frac{1}{2}\right)$ in Table. 4.1 . is same as the exact ground state energy for the Hubbard dimer at half filling. There is also no artificial transition to an anti-ferromagnetic solution like in Hartree-Fock case. But Müller functional does have a major flaw in predicting the magnetic structure of the ground state. It was found that several density matrices produces 
the same ground state energy for a given interaction strength $U$.

To analyze this degeneracy, we examined the natural orbitals obtained from the unbiased car-Parinello optimization. It was found that the natural orbitals differ from the ones in Eq. (4.11), only through a global spin rotation.

With the natural orbitals of Eq. (4.11), the total energy for the half-filled dimer obtained from Müller's functional can be expressed solely by the occupations as

$$
E^{M}=-2 t+\frac{1}{2} U+2 t\left(\sum_{\sigma} f_{a \sigma}\right)-\frac{1}{2} U \sum_{\sigma} \sqrt{f_{a \sigma} f_{b \sigma}} .
$$

The first two terms, which are independent of the occupations, are identical to the total energy Eq. (4.9) of the spin-restricted Hartree-Fock approximation. If only the bonding states are occupied, the remaining terms of Eq. 4.18 vanish and Müller's functional gives the same result as the Hartree-Fock approximation.

The minimization in this restricted space for a given interaction strength leads to a solution for occupations which lie on a line parametrized by $s$ as,

$$
\begin{aligned}
f_{a, \uparrow}^{M}(s) & =\frac{1}{1+R^{2}}+s, \\
f_{a, \downarrow}^{M}(s) & =\frac{1}{1+R^{2}}-s, \\
f_{b, \uparrow}^{M}(s) & =R^{2}\left(\frac{1}{1+R^{2}}+s\right), \\
f_{b, \downarrow}^{M}(s) & =R^{2}\left(\frac{1}{1+R^{2}}-s\right),
\end{aligned}
$$

where $R=4 t / U+\sqrt{1+(4 t / U)^{2}}$. The requirement, that the occupations remain between zero and one, limits the parameter $s$ to the interval

$$
s \in\left[-\frac{1}{R^{2}\left(1+R^{2}\right)}, \frac{1}{R^{2}\left(1+R^{2}\right)}\right] .
$$

The range of the occupations, which minimize the total energy Eq. (4.18), is shown in Fig. 4.2 as a function of interaction strength $U / t$. In the limit of infinite interaction strengths, we have $R=1$ respectively $s \in[-1 / 2,1 / 2]$ and the possible occupations $f_{a / b, \sigma}^{M}(s)=1 / 2+\sigma s$ cover the whole range from zero to one. The exact solution for the half fillied Hubbar dimer is a non-magnetic state, whereas the Müller functional produces a one dimensional manifold of degenerate magnetic states which have the same energy as the exact non-magnetic solution. This degeneracy leads to infinite susceptibility at zero temperature for the any finite interaction strength. However the exact solution at zero temperature has zero magnetic susceptibilty due to singlettriplet splitting (Ref. Sec. 2.7.1).

The magnetization of each site in the ground state of the Müller functional ob- 


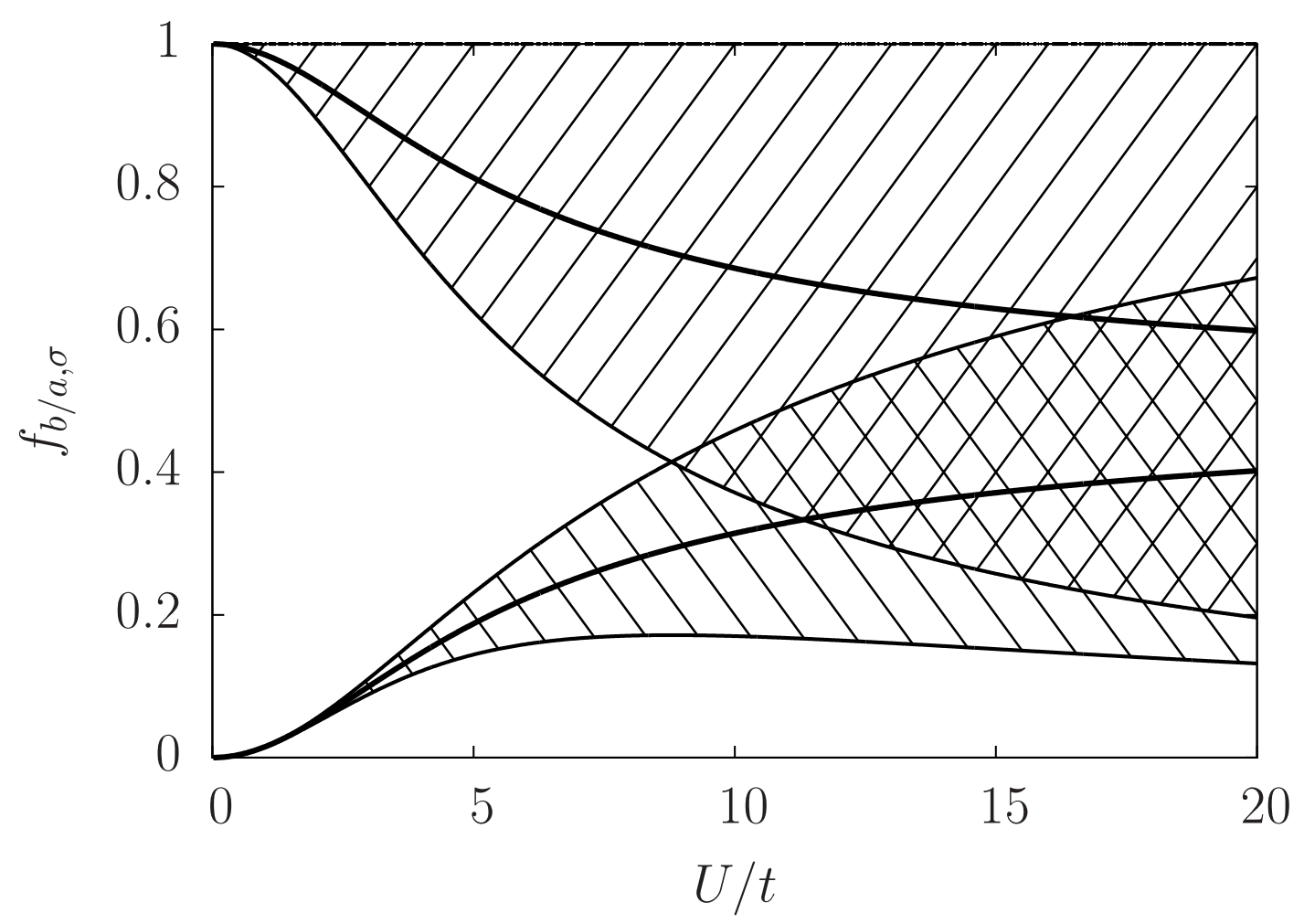

Figure 4.2: Occupations $f_{b, \sigma}$ and $f_{a, \sigma}$ from Eq. (4.19) of the half-filled dimer obtained with the Müller functional as a function of interaction strength $U / t$. The striped regions indicate the range of occupations in the manifold of degenerate groundstates. The thick solid lines indicate the mean values for the pair of occupations in the corresponding striped region. It also represents the degenerate occupations for the non-magnetic solution of the Müller functional. The occupations of the non-magnetic solution of the Müller functional coincide with those of the exact ground state of the Hubbard dimer.

tained with the occupations given by Eq. 4.19 has the form

$$
\begin{aligned}
m^{z}(s) & =\frac{1}{2}\left[f_{b, \uparrow}^{M}(s)-f_{b, \downarrow}^{M}(s)+f_{a, \uparrow}^{M}(s)-f_{a, \downarrow}^{M}(s)\right] \mu_{B} \\
& =\left(1+R^{2}\right) s \mu_{B}
\end{aligned}
$$

with the Bohr magneton $\mu_{B}$. It can assume any value with $\left|m_{z}\right|<1 / R^{2} \mu_{B}$. The symmetric solution $(s=0)$ only predicts the correct ground state which has zero magnetic moment. 


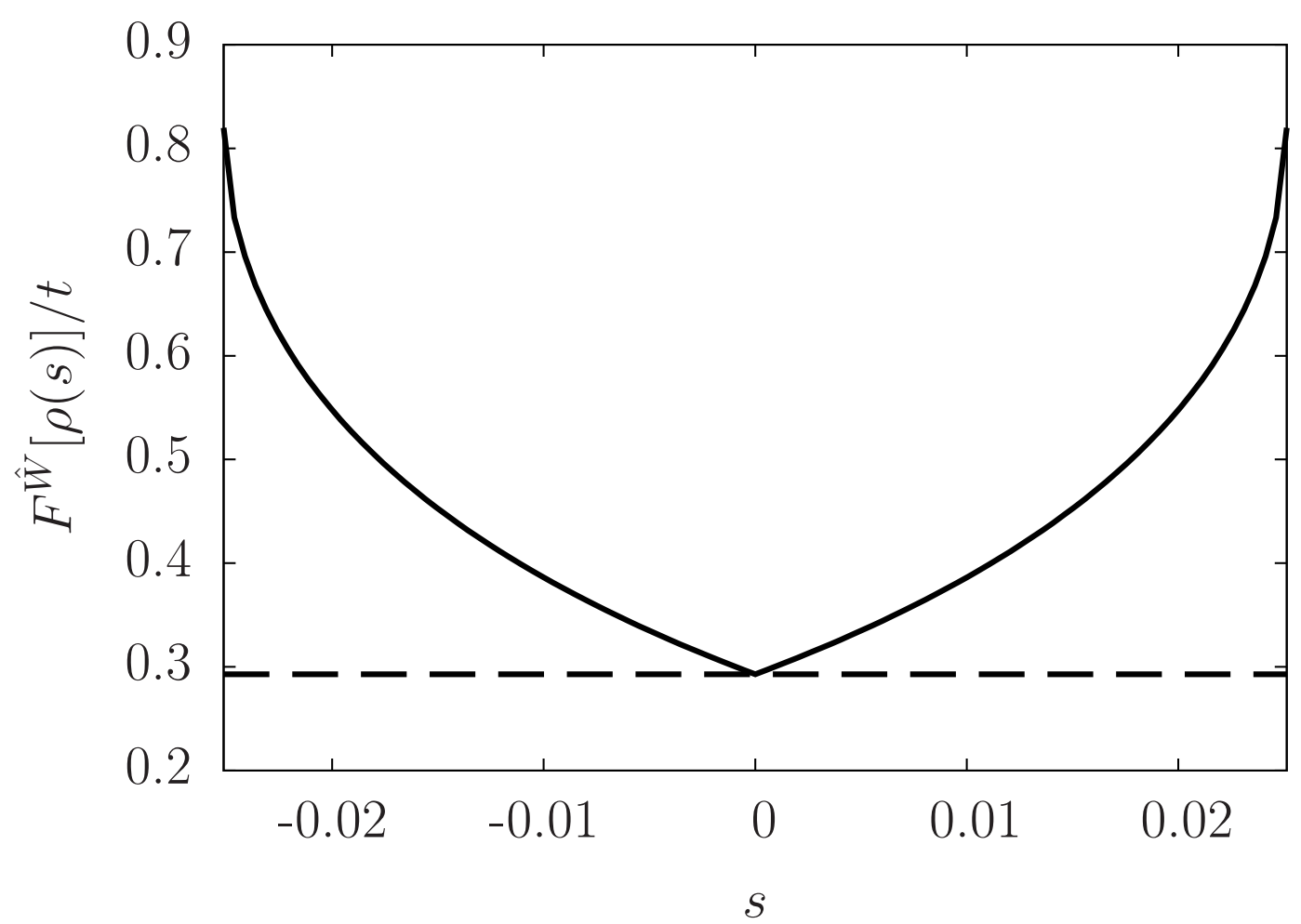

Figure 4.3: Müller's density-matrix functional (dashed line) and the exact functional (solid line) as a function of the line parameter $s$ for $U=4 t$. The density matrix $\rho(s)$ is given by Eq. 4.11 and Eq. 4.19. The values of the exact functional have been obtained by a constrained search over an ensemble of many-particle wave functions. The point $s=0$, where Müller's approximation and the exact functional coincide, corresponds to the symmetric solution $\left(m_{z}=0\right)$.

\section{Comparison to exact density matrix functional}

The exact density matrix functional for the Hubbard dimer at half filling for a given density matrix $\rho(s)$ living on the degenrate manifold of Eq.4.19), is evaluated using the full costrained search over an ensemble of many-particle wave functions [47] in Eq. 2.75).

The Müller functional is constant over the range of line parameter $s$ given by Eq. 4.20). As evident from Fig. 4.3, the enourmous difference in the functionals illustrates the severe problems of the Müller functional to describe the magnetic structure properly and indicates a systematic flaw in the functional. 


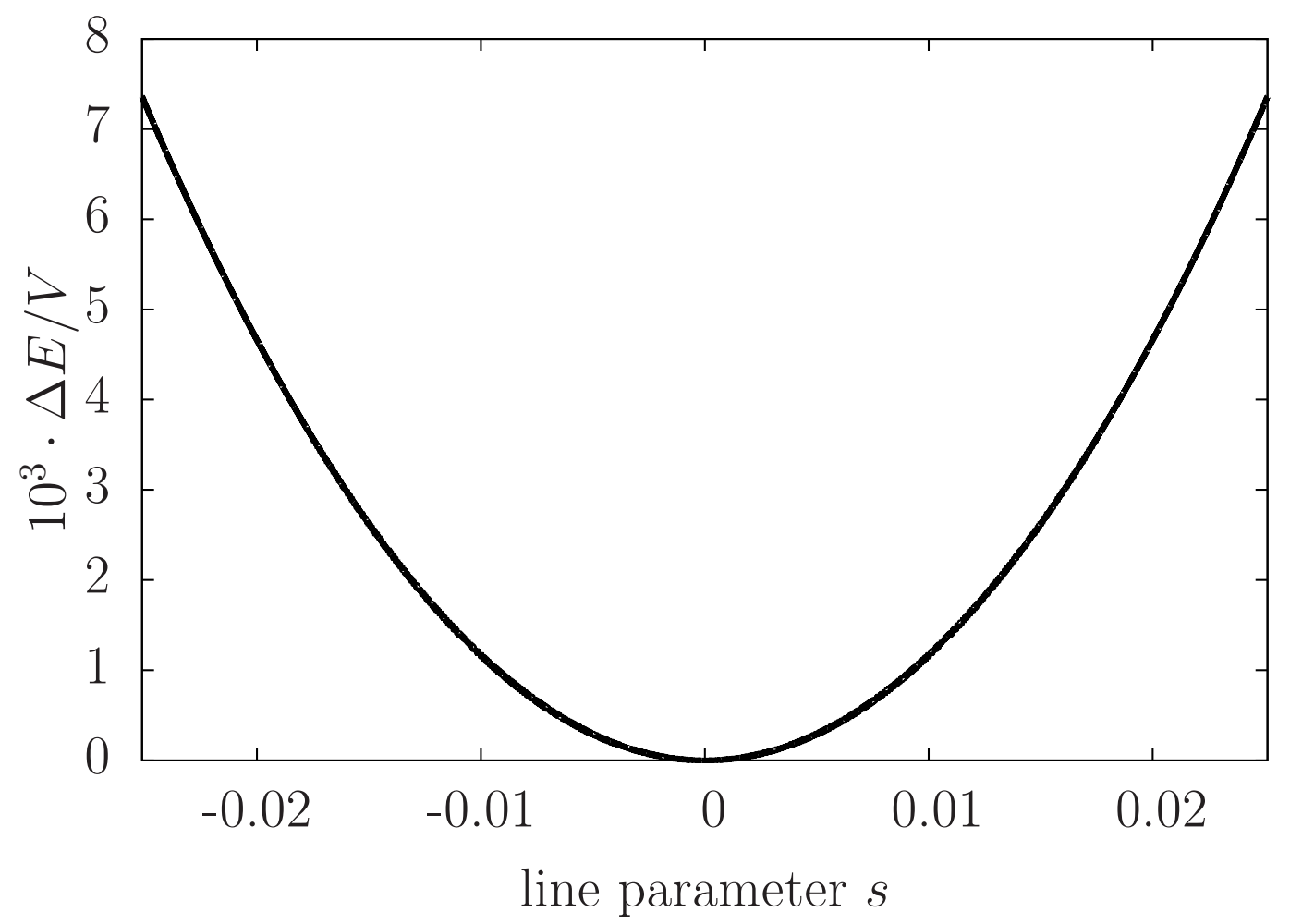

Figure 4.4: Energy $\Delta E=E[V, s]-E[V, s=0]$ of Eq. 4.23) of the Hubbard dimer obtained with the Müller functional including an off-site interaction in first-order perturbation theory with $U=4 t$ along the manifold Eq. (4.19) of ground states. The point $s=0$ indicates the non-magnetic solution.

\section{Off site interaction and the degeneracy breaking}

This severe problem of degeneracy or the infinite magnetic susceptibility raises the question whether the findings can be extended to more realistic systems. In this section we investigate the peculiarity of local Hubbard interaction which leads to the problem of degeneracy. To this end we extend the interaction in the Hubbard dimer to neighbouring site also as,

$$
\begin{aligned}
\hat{W} & =\frac{1}{2} \sum_{i} \sum_{\sigma, \sigma^{\prime}} U \hat{c}_{i, \sigma}^{\dagger} \hat{c}_{i, \sigma^{\prime}}^{\dagger} \hat{c}_{i, \sigma^{\prime}} \hat{c}_{i, \sigma} \\
& +\frac{1}{2} \sum_{i \neq j} \sum_{\sigma} V \hat{c}_{i, \sigma}^{\dagger} \hat{c}_{j, \sigma}^{\dagger} \hat{c}_{i, \sigma} \hat{c}_{j, \sigma} .
\end{aligned}
$$

where $V$ is the magnitude of interaction between same spin spin on the neighbouring site. In realistic system we do have extended interactions and therefore keeping the 
interaction local might limit the tranferability of findings of Hubbard dimer to real systems.

With the assumption that the interaction $V$ is small and the natural orbitals are the bonding and anti-bonding orbitals of Eq. (4.11), the energy landscape in the one dimensional degenerate manifold of Eq. 4.19), has the form,

$$
\begin{aligned}
E^{M}[V, s]= & E^{M}[0, s]+\frac{V\left(R^{2}-1\right)^{2}}{2} \\
& \times\left[\left(\frac{2}{1+R^{2}}\right)^{2}+s^{2}\right]+\mathcal{O}\left(V^{2}\right) .
\end{aligned}
$$

$E^{M}[0, s]$ is the $s$-independent total energy obtained with Müller's functional for the Hubbard dimer in the absence of an offsite interaction. It is given by Eq. 4.18) and Eq. 4.19.

As shown in Fig. 4.4, the energy in Eq. 4.23) due to a small offsite interaction $V$, has a minima at the $s=0$ i.e. at the symmetric solution with zero onsite magnetic moment. Therefore the artificial degeneracy or the infinite magnetic suscpetibility will not be apparent in real systems.

Nevertheless, as evident from the comparison with the exact functional shown in Fig. (4.3), the changes produced by the off-site term are far too small: In order to produce an energy difference between the maximally polarized state (see Eq. 4.20) and the unpolarized state comparable to the exact result shown in Fig. 4.3, an unrealistically large offsite interaction parameter of order $V=10 t$ would be required.

\subsection{Power functional}

The ground state energy obtained from unbiased Car-Parinello optimization, for the power functional i.e. $\left(c_{m n}(\alpha)\right)$ in the Table. 4.1, is shown in Fig. 4.1. In the calculations the value of $\alpha$ was chosen to be 0.53 . There is an anti-ferromagnetic transition after certain critical value of interaction strength. The occupations of the Hubbard dimer obtained from power functional $(\alpha=0.53)$ is shown in Fig. 4.5 .

Whereas the density-matrix functional in the Hartree-Fock approximation produces integer and pairwise identical occupations, the power functional produces fractional occupations which are not identical in pairs. Near $U=6 t$, we observe a transition. This transition separates the Müller-like behavior (fractional occupations) at small interactions from a Hartree-Fock-like behavior (integer occupations) at large interactions.

- At small interactions, the solutions are analogous to those of the Müller functional. However, from the manifold of degenerate ground states of the Müller functional, the power functional favors the state with maximal ferromagnetic moments i.e. the extreme of . 


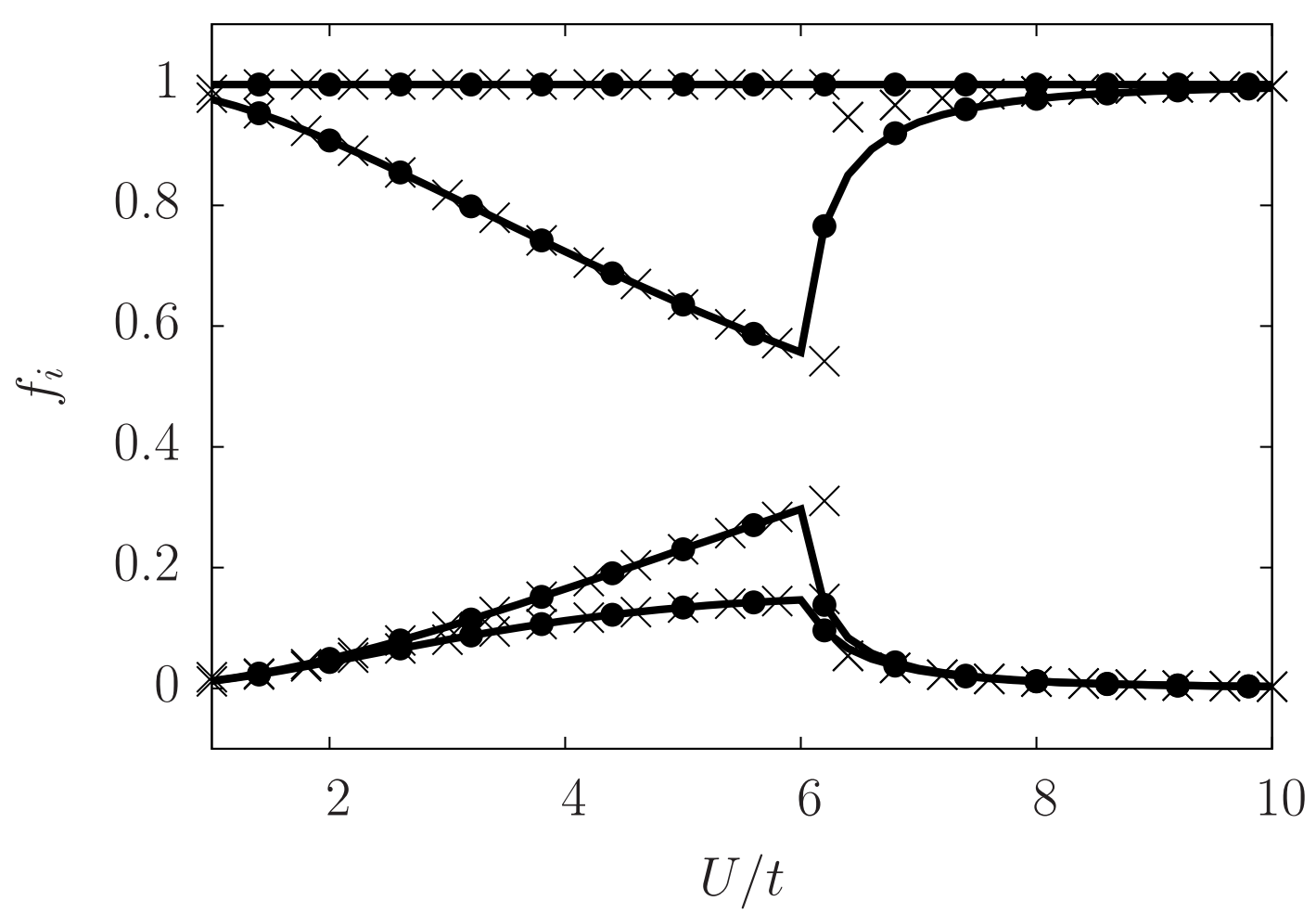

Figure 4.5: Occupations $f_{i}$ as a function of $U / t$ for the power functional with $\alpha=0.53$. Solid dots have been obtained from an unbiased optimization of the power functional. The solid lines are obtained from a restricted optimization using the non-collinear natural orbitals of the ansatz Eq. 4.27). The diagonal crosses are obtained from a restricted optimization using the collinear natural orbitals Eq. 4.26) of the Hartree-Fock approximation.

- At larger interactions, the ground state undergoes a transition into a noncollinear ground state. For very large interaction the state approaches the Hartree-Fock-like antiferromagnetic state.

\section{Ferromagnetic solution in the weakly interacting regime:}

The occupations in the weakly interacting regime can be understood as follows: In case of the Müller density-matrix functional, we have shown in Sec. 4.4.2 that there exists a manifold of degenerate ground-state density matrices on the line given by Eq. 4.19. If we increase the parameter $\alpha$ of the power functional infinitesimally as $\alpha=\frac{1}{2}+\epsilon$ where $\epsilon>0$, and restrict ourselves to interaction strengths $U / t$ where the natural orbitals are bonding and antibonding states, Eq. (4.11), the total energy 
along the line given by Eq. (4.19) is

$$
\begin{aligned}
E_{\alpha=\frac{1}{2}+\epsilon}^{P}(s) & =2 t\left(\frac{2}{1+R^{2}}-1\right)+U \\
& -\frac{U}{4} \sum_{\sigma= \pm 1}\left(1+R^{1+2 \epsilon}\right)^{2}\left(\frac{1}{1+R^{2}}+\sigma s\right)^{1+2 \epsilon}
\end{aligned}
$$

where $R=4 t / U+\sqrt{1+(4 t / U)^{2}}$. The energy in Eq. 4.24), shown in Fig. 4.6, has a negative curvature along the line parameter $s$ and the minima lie at the boundaries given in Eq. 4.20).

At these boundaries, the extreme non-symmetric solutions of the Müller functional, one of the states is always fully occupied (See Fig. 4.2) because this maximum occupation limits the range of degenerate solutions. This explains the corresponding observation in Fig. 4.5.

Unfortunately, any change of the parameter $\alpha$ away from the value of the Müller functional, destroys the non-magnetic ground state in favor of an unphysical ferromagnetic state.

\section{Large-interaction regime}

The Hartree-Fock approximation exhibits a transition from a non-magnetic state to an antiferromagnetic state at $U=2 t$. This transition is absent in the Müller functional, but it is present in the power functional for all other values of $\alpha>\frac{1}{2}$.

In order to explore, how the power functional interpolates between these two extreme cases, we calculated the product $\left\langle\hat{\vec{S}}_{1}\right\rangle \cdot\left\langle\hat{\vec{S}}_{2}\right\rangle$ of the spin expectation values at the two sites of the dimer. A positive value of $\left\langle\hat{\vec{S}}_{1}\right\rangle \cdot\left\langle\hat{\vec{S}}_{2}\right\rangle$ corresponds to a ferromagnetic, a negative value to an antiferromagnetic spin alignment. The maximum absolute value is $\hbar^{2} / 4$.

For the Hubbard dimer at half filling, $\left\langle\hat{\vec{S}}_{1}\right\rangle \cdot\left\langle\hat{\vec{S}}_{2}\right\rangle$ is shown in Fig. 4.7 as function of interaction strength $U / t$ and the parameter $\alpha$ of the power functional. For the Müller functional discussed in Sec. 4.4 .2 , i.e. for $\alpha=1 / 2$, we consider the solution with the strongest polarization, because this is the state that continuously matches to the solutions of the power functional. In this ferromagnetic state, $\left\langle\hat{\vec{S}}_{1}\right\rangle \cdot\left\langle\hat{\vec{S}}_{2}\right\rangle$ is positive. Unfortunately, the correct non-magnetic state is not a ground state of the power functional for $\alpha>\frac{1}{2}$.

At a critical interaction strength $U_{c}(\alpha)$ the power functional exhibits a transition from this ferromagnetic state into a complex non-collinear state with a mostly antiferromagnetic spin alignment. The angle between the magnetization on the two sites is shown in Fig. 4.8.

Fig. 4.7 clearly shows the location of the transition between the ferromagnetic and the antiferromagnetic non-collinear regime. The critical interaction strength 


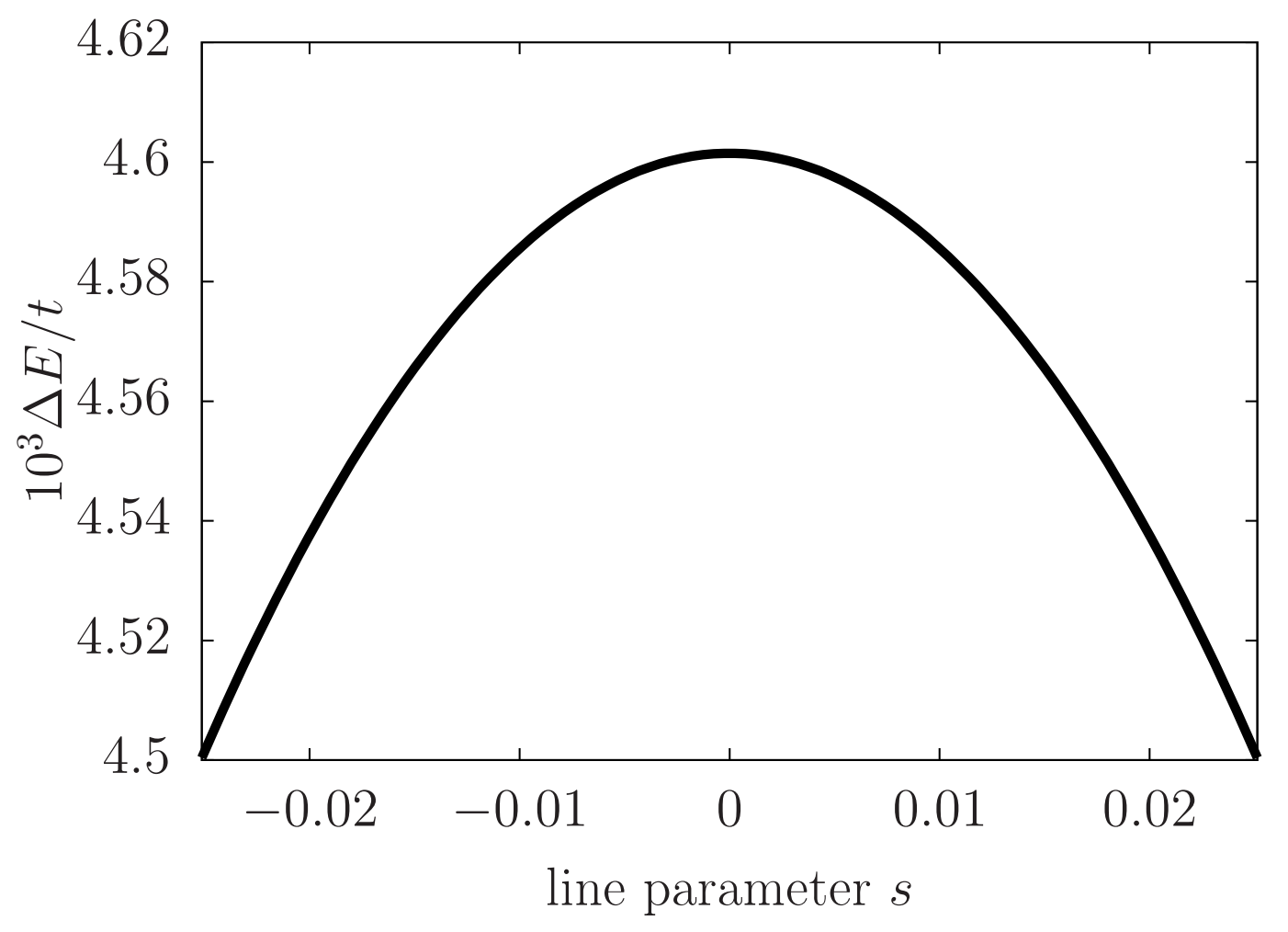

Figure 4.6: Total energy difference $\Delta E=E_{\alpha=1 / 2+\epsilon}^{P}[\rho(s)]-E_{\alpha=1 / 2}^{P}[\rho(s)]$ given by Eq. 4.24 for $U=4 t$ using the power functional approximation with $\epsilon=10^{-3}$ as a function of the line parameter $s$ that parametrizes the one-particle reduced density matrix according to Eq. (4.19).

$U_{c}(\alpha)$ of this transition is infinite for the Müller functional. As the parameter $\alpha$ is increased, the critical interaction strength falls off rapidly and approaches the value $U_{c}(\alpha=1)=2 t$ of the Hartree-Fock approximation.

Thus, the power functional exhibits a Hartree-Fock-like transition into an antiferromagnetic ground state except for the limiting case, the Müller functional. By choosing the parameter $\alpha$ sufficiently close to $1 / 2$, the transition can be shifted into a regime that is physically less important.

\section{Collinear approximation using the Hartree-Fock natural orbitals}

In order to get a qualitative understanding of the asymmetric occupations (Ref. Fig. 4.5 and the critical value of interaction strength $U_{c}$ of the transition to antiferromagnetic solutions (Ref. Fig. 4.7), we use an ansatz that covers both extreme cases, namely the Müller functional with $\alpha=\frac{1}{2}$ and the Hartree-Fock approximation with $\alpha=1$. These are, one the one hand, the asymmetric natural orbitals 
Eq. 4.10 that can describe the antiferromagnetic state of the Hartree-Fock approximation. On the other hand, the ansatz allows for fractional occupations to capture the nature of the ground state of the Müller functional.

With this ansatz, the one-particle reduced density matrix $\rho\left(f_{1}, \ldots, f_{4}, \gamma\right)$ is a function of occupations $f_{n}$ and the angle $\gamma$ and the corresponding total energy $E^{P, \alpha}$ obtained with the power functional is

$$
\begin{aligned}
E_{\alpha}^{P}\left[\rho\left(f_{1}, \ldots, f_{4}, \gamma\right)\right] & =E^{\mathrm{kin}}\left[\rho\left(f_{1}, \ldots, f_{4}, \gamma\right)\right] \\
& +F_{\alpha}^{P}\left[\rho\left(f_{1}, \ldots, f_{4}, \gamma\right)\right]
\end{aligned}
$$

where

$$
\begin{aligned}
E^{\mathrm{kin}}\left[\rho\left(f_{1}, \ldots, f_{4}, \gamma\right)\right] & =-t \cos (2 \gamma)\left(f_{1}+f_{2}-f_{3}-f_{4}\right) \\
F_{\alpha}^{P}\left[\rho\left(f_{1}, \ldots, f_{4}, \gamma\right)\right] & =\frac{U}{4}\left[\left(f_{1}+f_{2}+f_{3}+f_{4}\right)^{2}-\left(f_{1}^{\alpha}+f_{3}^{\alpha}\right)^{2}-\left(f_{2}^{\alpha}+f_{4}^{\alpha}\right)^{2}\right] \\
& +\frac{U}{4} \sin ^{2}(2 \gamma)\left[\left(f_{2}+f_{3}-f_{4}-f_{1}\right)^{2}-\left(f_{1}^{\alpha}-f_{3}^{\alpha}\right)^{2}-\left(f_{2}^{\alpha}-f_{4}^{\alpha}\right)^{2}\right] .
\end{aligned}
$$

An approximation, which is a strict upper bound, for the total energy of the power functional is obtained by minimizing Eq. 4.26 for a half-filled system with occupations between zero and one.

As a characteristic example, the resulting occupations for $\alpha=0.53$ are shown in Fig. 4.5. The properties of this ansatz with regard to the description of the transition to the antiferromagnetic state will be investigated in the following section after a more general discussion of the transition.

The ansatz using the collinear natural orbitals Eq. (4.10) and arbitrary occupations is, however, not able to describe the true ground state for the power functional in the strongly interacting regime. The energy difference of the ansatz to the unbiased solution is shown in Fig. 4.9. The deviation is largest near the transition. The transition point is slightly displaced by the collinear ansatz, which explains the sharp rise. For larger interactions, the error due to the collinear approximation falls off rapidly. It should be noted that the overall error due to the restricted ansatz is apparently small. For the parameter $\alpha=0.53$ used in Eq. (4.9), the maximum error in the energy is less than $1 \%$ of the binding energy.

\section{Beyond the collinear approximation}

The ansatz using the Hartree-Fock natural orbitals already gives a fairly good description of the ground state of the power functional. How do the natural orbitals of the power functional differ from those of the Hartree-Fock solution?

In the large interaction region, the power functional produces non-collinear ground states. The natural orbitals of the power functional can be represented as superpo- 


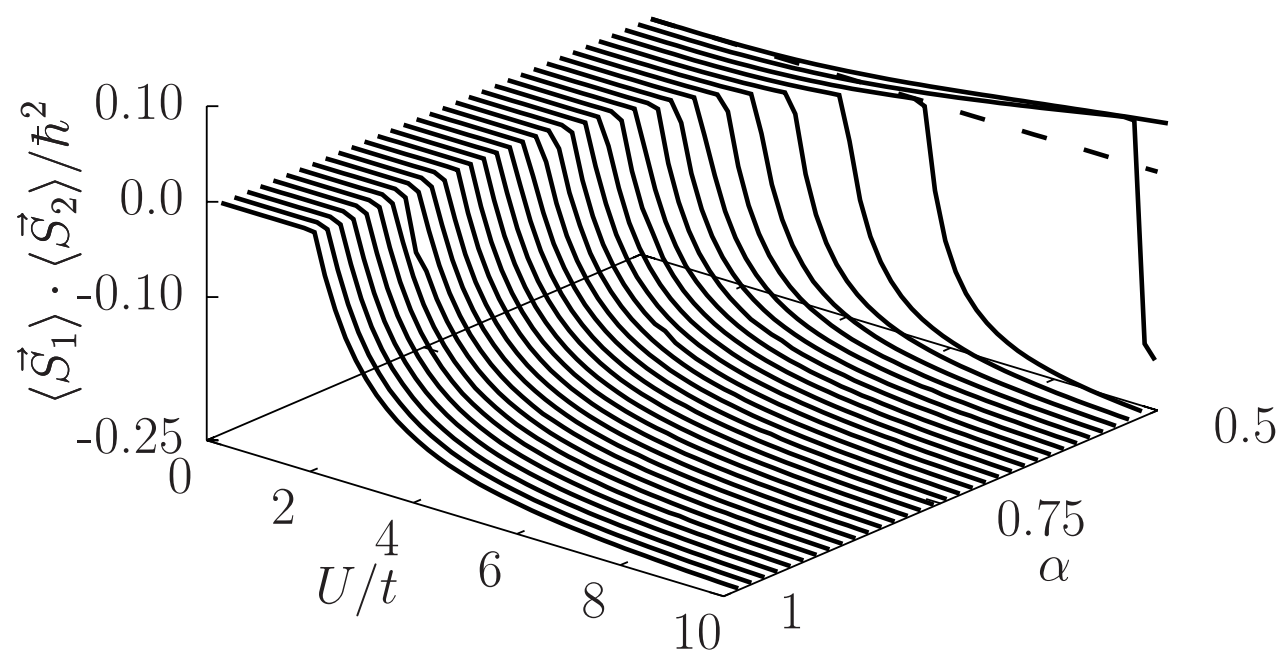

Figure 4.7: Scalar product $\left\langle\hat{\vec{S}}_{1}\right\rangle \cdot\left\langle\hat{\vec{S}}_{2}\right\rangle$ of the spin expectation vectors on the two sites of the Hubbard dimer as an indicator for the transition to the antiferromagnetic state within the power functional approximation with the parameter $\alpha$ for the Hubbard dimer at various interaction strengths. A positive value indicates a ferromagnetic state, a negative value an antiferromagnetic state. For the Müller functional, i.e. $\alpha=1 / 2$, the dashed line represents the result for the symmetric solutions and the solid line the corresponding degenerate result for the degenerate maximally polarized state.

sitions of bonding and antibonding states,

$$
\begin{aligned}
\left|\phi_{1}^{P}\right\rangle & =|b, \uparrow\rangle \cos \left(\beta_{1}\right)-|a, \downarrow\rangle \sin \left(\beta_{1}\right) \\
\left|\phi_{2}^{P}\right\rangle & =|b, \downarrow\rangle \cos \left(\beta_{2}\right)-|a, \uparrow\rangle \sin \left(\beta_{2}\right) \\
\left|\phi_{3}^{P}\right\rangle & =|b, \uparrow\rangle \sin \left(\beta_{1}\right)+|a, \downarrow\rangle \cos \left(\beta_{1}\right) \\
\left|\phi_{4}^{P}\right\rangle & =|b, \downarrow\rangle \sin \left(\beta_{2}\right)+|a, \uparrow\rangle \cos \left(\beta_{2}\right) .
\end{aligned}
$$

The two angles $\beta_{1}$ and $\beta_{2}$ are free variational parameters. The natural orbitals of the non-interacting system, respectively those of the Müller functional are obtained with $\beta_{1}=\beta_{2}=0$. The values of the two parameters are shown in Fig. 4.10 for one example of the power functional.

In the Hartree-Fock approximation, respectively in the power functional with the collinear ansatz, the pair of bonding and antibonding orbitals that contribute to a 


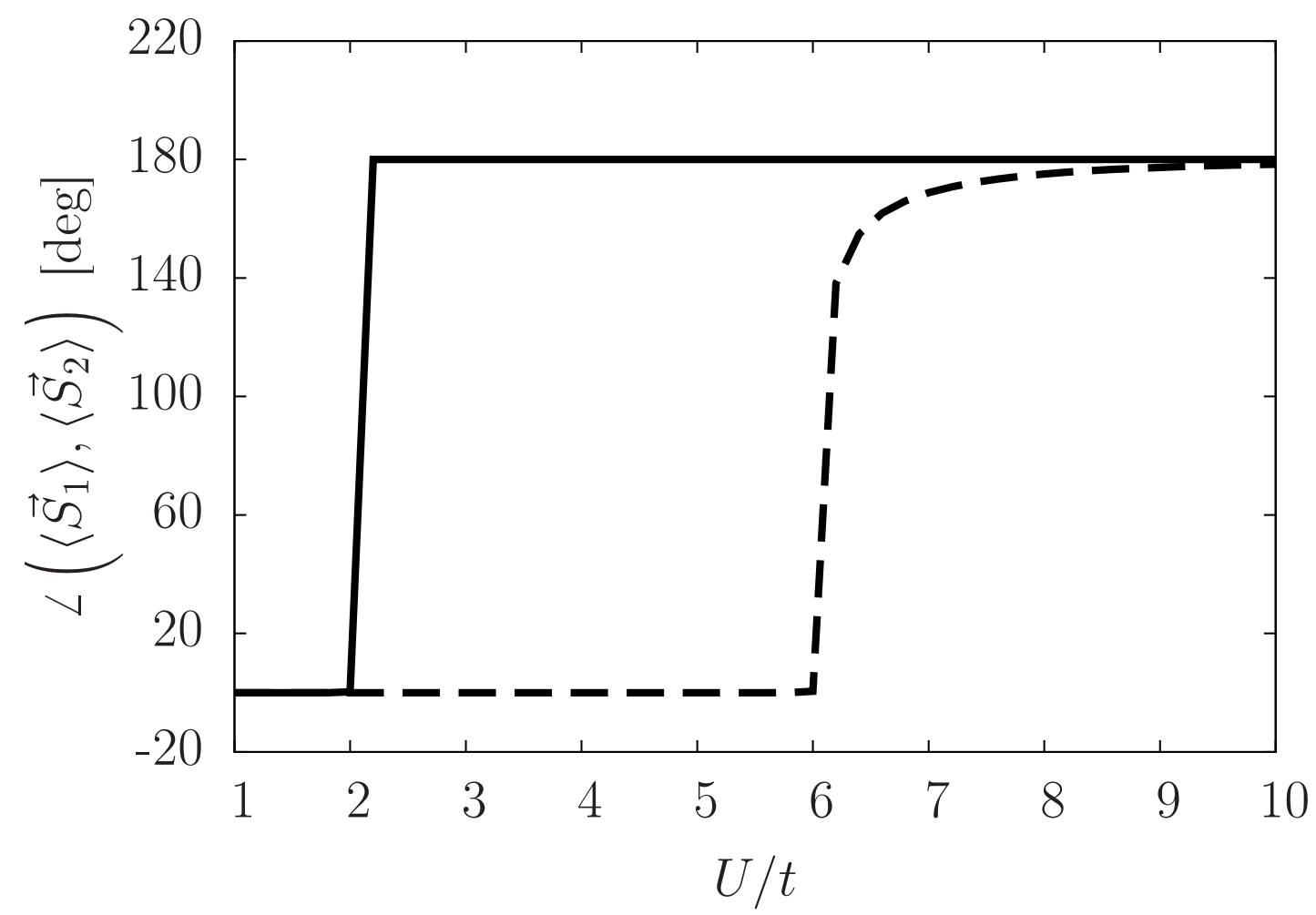

Figure 4.8: Angle between the spin expectation vectors $\left\langle\vec{S}_{1}\right\rangle$ and $\left\langle\vec{S}_{2}\right\rangle$ on the two sites of the Hubbard dimer as function of the interaction strength $U$. Dashed line: power functional with the parameter $\alpha=0.53$; solid line: Hartree-Fock approximation. This angle is a measure of collinearity of natural orbitals.

natural orbital, given in Eq. 4.10, have the same spin direction. This results in the localization of the electron on one or the other site of the dimer. The emerging picture is appealing because it reflects the left-right correlations of the electrons. The admixture of antibonding states to the natural orbitals for the two spin directions is the same. Thus, there is no symmetry-breaking charge disproportionation.

The natural orbitals Eq. (4.27) of the power functional are composed of bonding and antibonding orbitals with opposite spin directions. This leads to natural orbitals with equal weight on both sites, but the spins on both sides have a finite angle between them. The state has an intrinsically non-collinear, even though still a coplanar spin structure.

The admixture of antibonding states in the two pairs is independent in the power functional, so that the natural orbitals contain two independent free parameters, namely $\beta_{1}$ and $\beta_{2}$.

The net magnetic moment of each of the four natural orbitals points along the same direction. For the choice in Eq. (4.27), this is the $z$-direction. The parameters 
$\beta_{1}$ and $\beta_{2}$ control the relative angles of the local moments on the two sites of the dimer for each of the natural orbitals. This angle is $4 \beta_{1}$ for the orbitals $\left|\phi_{1}^{P}\right\rangle$ and $\left|\phi_{3}^{P}\right\rangle$ and it is $4 \beta_{2}$ for the orbitals $\left|\phi_{2}^{P}\right\rangle$ and $\left|\phi_{4}^{P}\right\rangle$. The natural orbitals are pairwise antiparallel: On any given site $\left|\phi_{1}^{P}\right\rangle$ and $\left|\phi_{3}^{P}\right\rangle$ have local moments in opposite directions. Similarly, this holds for $\left|\phi_{2}^{P}\right\rangle$ and $\left|\phi_{4}^{P}\right\rangle$.

It seems that the ground states of the power functional do not connect continuously to those of the Hartree-Fock approximation, because the natural orbitals belong to different classes. This is, however, not so: The ansatz for the natural orbital Eq. 4.27) connects smoothly to those of the Hartree-Fock approximation in Eq. 4.10 when the two parameters $\beta_{1}$ and $\beta_{2}$ become equal, and furthermore the occupations become integer. This limit of the ansatz Eq. 4.27) for the power functional describes, however, an antiferromagnet with the local moments aligned along the $x$-direction, while the ansatz of Eq. 4.10 for the Hartree-Fock solution is polarized along the z-direction. Thus they are related by a global spin rotation, which is a symmetry of the Hamiltonian.

\subsection{Beyond half-filling}

Up to now, we considered only the half filled case of the Hubbard dimer. Here we consider also deviations from the particle number $N=2$.

To avoid mathematical complications, we define $E(N)$ thermodynamically consistent as the zero-temperature limit of the Helmholtz potential $\beta \rightarrow \infty$, which in turn is constructed from the grand potential by a Legendre-Fenchel transform

$$
E(N)=\lim _{\beta \rightarrow \infty} \max _{\mu}\left[-\frac{1}{\beta} \ln \left(\operatorname{Tr} \mathrm{e}^{-\beta(\hat{H}-\mu \hat{N})}\right)+\mu N\right] .
$$

The trace is performed over the fermionic Fock space.

It can be shown that the total energy $E(N)$ consists of piecewise linear segments between integer particle numbers. Thus the slope of the total energy $E(N)$, the chemical potential $\mu=d E / d N$, is usually ${ }^{1}$ discontinuous at integer occupations. This derivative discontinuity gives the fundamental band gap which is defined as the difference of electron affinity and ionization potential. The band gap is relevant, not only as an estimation related to optical spectra, but, more importantly, for the response functions and chemical equilibria. Therefore, we investigate whether the derivative discontinuities are properly described by the approximate density-matrix functionals.

The total energy $E(N)$ of the exact solution and several power functionals is shown in Fig. 4.11 and the corresponding chemical potential in Fig. 4.12, For the

\footnotetext{
${ }^{1}$ For degenerate states and when the electron addition and removal are dominated by delocalized states the discontinuity may also vanish or become infinitesimally small.
} 


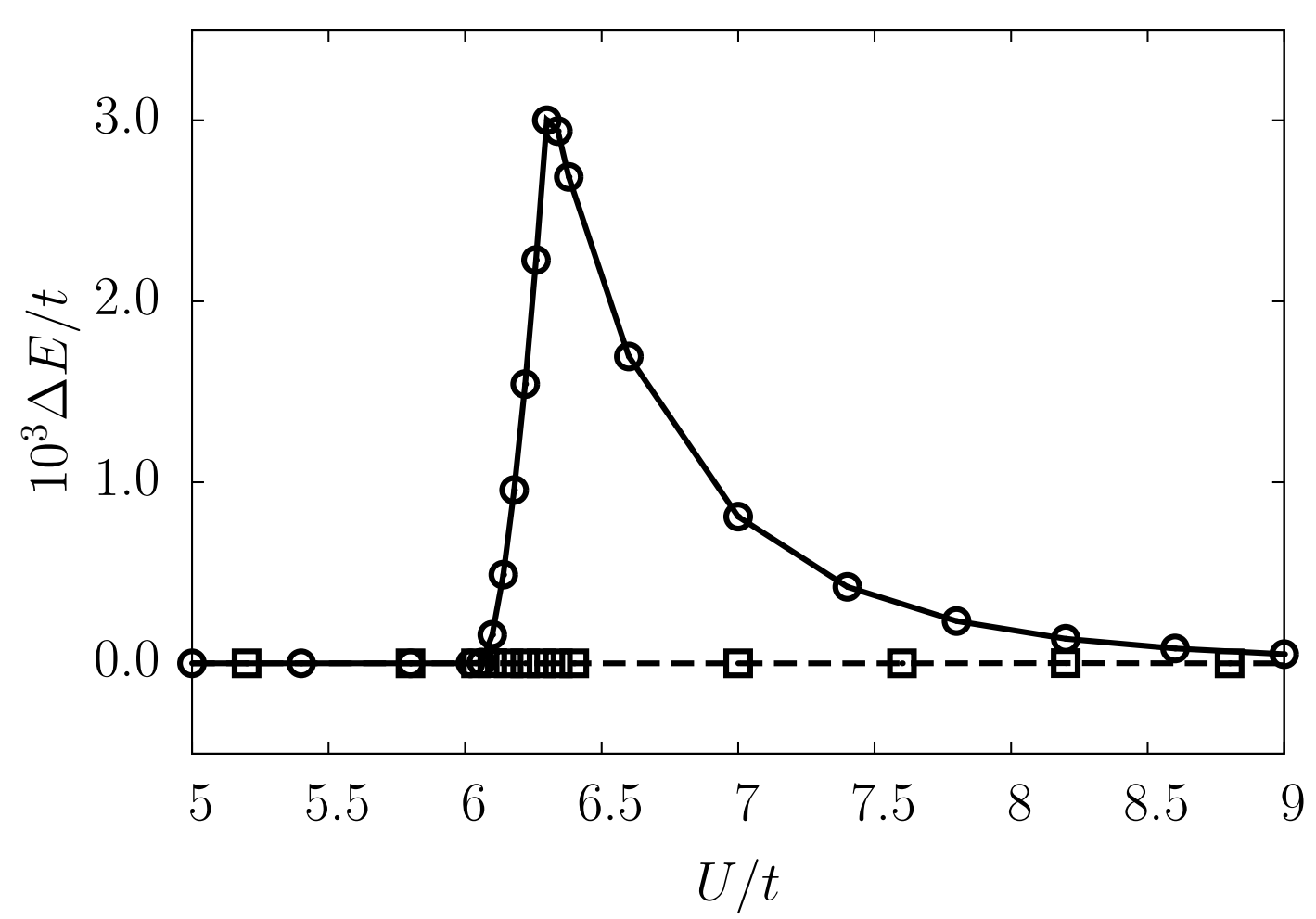

Figure 4.9: Energy difference $\Delta E$ of the power functional with $\alpha=0.53$ between the density matrices obtained by a constrained and an unbiased optimization. $\Delta E$ for the case of constrained optimization with the natural orbitals of a Hartree-Fock in Eq. (4.10) is the solid line with circle symbols, while the $\Delta E$ obtained from constrained optimization with the non-collinear natural orbitals of Eq. 4.27) is the dashed line with square symbols.

Hubbard dimer, the derivative discontinuity at $N=2$ is due to a combination of the one-particle gap and the interaction. The derivative discontinuity at $N=1$ is, however, entirely due to the interaction. These features are clearly visible for the exact calculation shown in Fig. 4.11.

In the Hartree-Fock approximation, the energy for fractional occupations has a negative curvature for $1<N<3$. As a result, the derivative discontinuities are larger than in the exact solution. It reflects the well known observation that HartreeFock calculations overestimate band gaps. This observation can be rationalized with a lack of screening in the Hartree-Fock approximation that reduces the effective interaction strength.

The Müller functional, however, fails to give any derivative discontinuity. It is surprising, that a functional that performs as well as the Müller functional for $N=2$ is completely unable to capture the correct physics beyond half filling. It adds to 


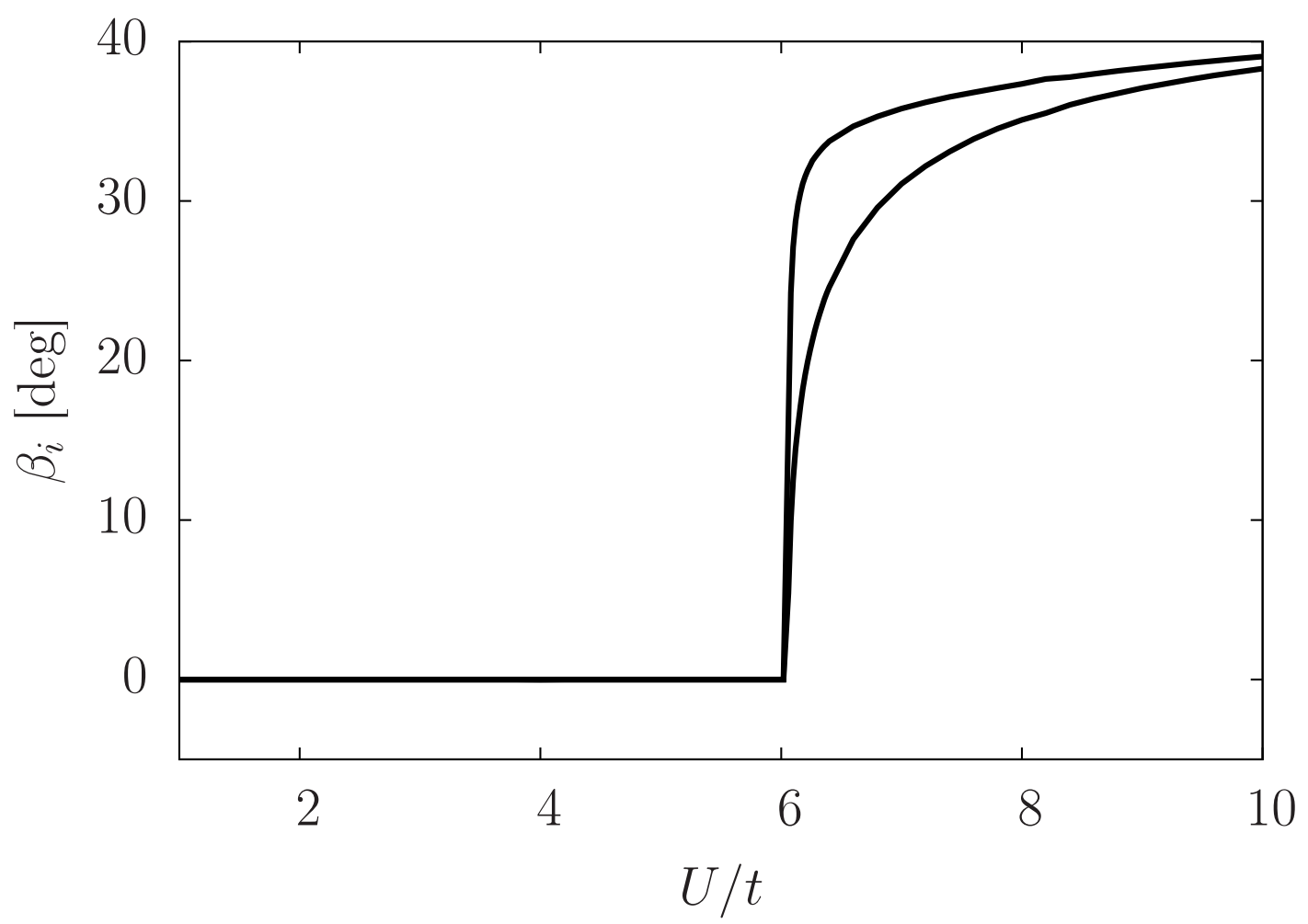

Figure 4.10: Parameters $\beta_{1}$ and $\beta_{2}$ defining the natural orbitals Eq. 4.27) of the power functional for $\alpha=0.53$ as function of the interaction strength.

the simplified picture that the Müller functional behaves very metal-like: It does not have a band gap and and its magnetic susceptibility is infinite.

Except for the Hartree-Fock limit, also the power functional lacks a derivative discontinuity. This is apparent from Fig. 4.12. For small $\alpha$, that is the Müller-like regime, the power functional behaves analogous to the Müller functional itself. In the parameter regime of the antiferromagnetic ground state, however, the chemical potential makes a continuous transition between two distinct linear functions of the particle number.

This behavior of the power functional for the Hubbard dimer is analogous to that observed earlier for finite [89, 90] and extended systems [53, 91].

In order to extract values for the band gap despite of the absence of a derivative discontinuity, Sharma et al. 53. proposed the extrapolation method, which exploits the behavior of $E(N)$ further away from the Fermi level. Sharma et al. exploit that the chemical potential makes a transition between two linear functions. The extrapolation of these linear functions to the integer particle number yields an offset which is identified with the band gap. This method yields finite band gaps in the appropriate parameter range of the power functional, where the Müller func- 
tional incorrectly predicts a vanishing band gap [53]. Surprisingly, the band gaps obtained using the extrapolation method from the power functional agree well with experimental results even for non-magnetic calculations.

Our results for the Hubbard dimer shown in Fig. 4.12 indicate that the band gap obtained with the extrapolation method[53] can be tuned between zero and the Hartree-Fock value by adjusting $\alpha$. Signatures of this behavior have been observed in studies that investigated the dependence on the power functional parameter $\alpha$ for realistic systems [53, 90].

The absence of a true derivative discontinuity using the power functional and the tunability of the band gap determined with the extrapolation method is not limited to the antiferromagnetic ground state. As shown in Fig. 4.13, the Hubbard dimer behaves qualitatively similar, when the spin polarization is suppressed. In the nonmagnetic calculations, the onset of a finite band gap obtained by the extrapolation method is delayed to larger power parameters $\alpha$. This finding is analogous to that observed for $\mathrm{NiO}$, for which non-magnetic calculations find a metallic ground state for $\alpha<0.65$ [92], whereas non-collinear calculations find an insulating ground state already for $\alpha=0.56[93]$.

\subsection{Beyond the dimer}

The question remains whether the findings for the Hubbard dimer persist in larger systems with more degrees of freedom. This is relevant for calculations of more complex systems having large unit cells. For this purpose we performed calculations for the power functional for Hubbard rings and Hubbard chains.

Figure 4.14 shows the occupation numbers for a half-filled Hubbard ring at an intermediate interaction strength of $U=5 t$, which like the Hubbard dimer, has an antiferromagnetic ground state in the Hartree-Fock approximation. For the Müller functional we obtain fractional occupations as for the dimer. While the fractional occupations deviate from the exact result, their deviation from integer occupations are of the same order of magnitude as in the exact solution. The power functional exhibits abrupt transitions to an antiferromagnetic state around $\alpha_{c} \approx 0.58$ very analogous to the Hubbard dimer.

For a six-site Hubbard chain with seven electrons, i.e. one electron more than half filling, the pattern of transitions is even more complex: This behavior is shown in Figure 4.15. There are now three transitions:

1. A continuous transition between $\alpha \approx 0.567$ and $\alpha \approx 0.569$ from the nonmagnetic Müller ground state to a state with collinear spins in the pattern $\uparrow \downarrow \uparrow \uparrow \downarrow \uparrow$, which is only stable in a small window of parameters.

2. Around $\alpha \approx 0.576$ there is a non-smooth transition to a state with an antiferromagnetic spin-structure, i.e. $\uparrow \downarrow \uparrow \downarrow \uparrow \downarrow$. 


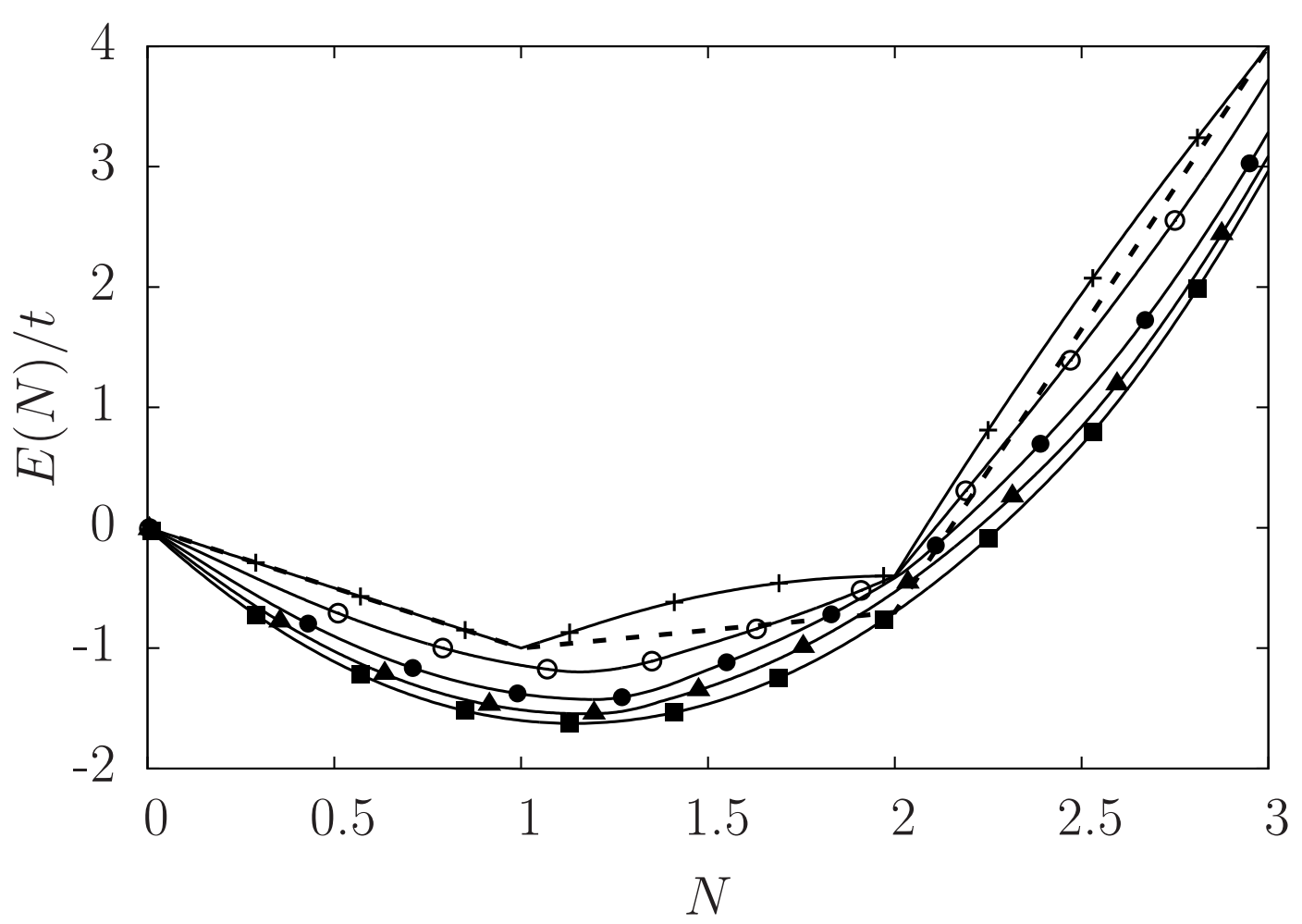

Figure 4.11: Ground-state energy $E(N)$ of the Hubbard dimer with $U=5 t$ in units of the hopping parameter $t$ as function of particle number $N$. The critical power functional parameter for the transition to an antiferromagnetic state lies at $\alpha \approx 0.54$ for the given interaction strength. Dashed line: exact solution, crosses: Hartree-Fock approximation, open circles: power functional with $\alpha=0.7$, filled circles: power functional with $\alpha=0.58$, triangles: power functional with $\alpha=0.53$, squares: Müller functional.

3. Beyond $\alpha \approx 0.75$, the antiferromagnetic structure breaks up and evolves into the HF-ground state having a spin-structure given by $\uparrow \downarrow \uparrow \uparrow \downarrow \uparrow$.

These examples demonstrate that the power functional can generate a variety of magnetic states even for simple systems.

\subsection{Summary}

The popular density-matrix functionals, the Müller functional[50], the Hartree-Fock approximation and the power functional[53], which continuously interpolates between the other two, have been benchmarked for the Hubbard dimer.

Particular emphasis has been given to left-right correlation, the dominant correla- 


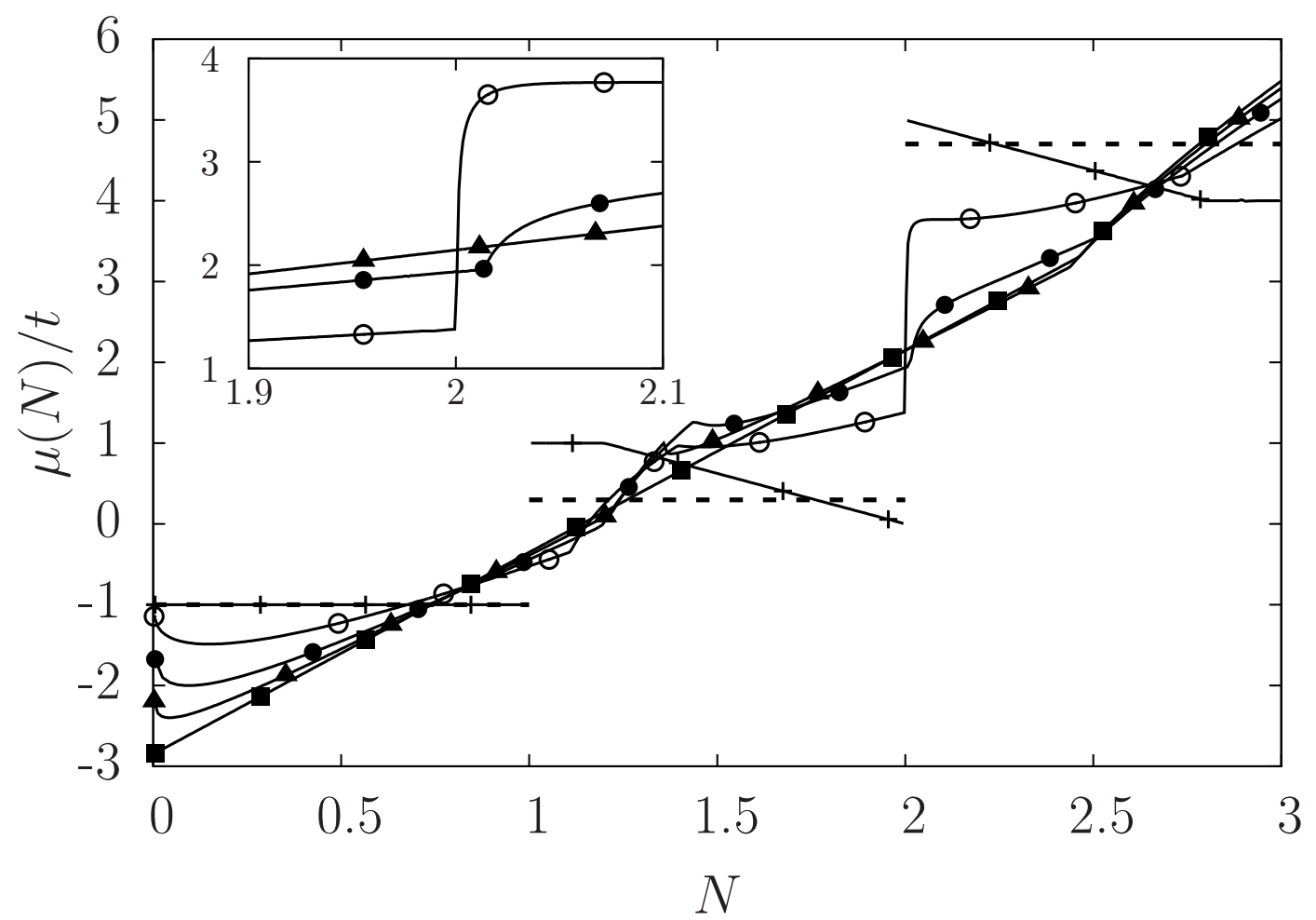

Figure 4.12: Chemical potential $\mu(N)$ of the Hubbard dimer with $U=5 t$ in units of the hopping parameter $t$ as function of particle number $N$. The behavior of the power functional with $1 / 2<\alpha<1$ close to half-filling is shown in the inset. Dashed line: exact solution, crosses: Hartree-Fock approximation, open circles: power functional with $\alpha=0.7$, filled circles: power functional with $\alpha=0.58$, triangles: power functional with $\alpha=0.53$, squares: Müller functional.

tion effect for bond dissociation, which is not captured in local density functionals [28]. Left-right correlation describes that electrons localize on opposite sites of the dimer. This electron correlation, which increases with the interaction strength, avoids the energetic cost of the Coulomb repulsion due to double occupancy of a site. In the Hartree-Fock approximation, this left-right correlation leads to an antiferromagnetic state with a spin-up electron mostly localized on one side and the spin-down electron on the other. This so-called broken-symmetry state disagrees with the exact solution, which is a singlet state, having no local moments, but nevertheless antiferromagnetic correlations similar to the broken symmetry state. More importantly, however, the antiferromagnetic transition is an abrupt one and not a continuous buildup of antiferromagnetic correlations as in the exact solution. The result is a qualitatively incorrect shape of the total energy during bond dissociation. 


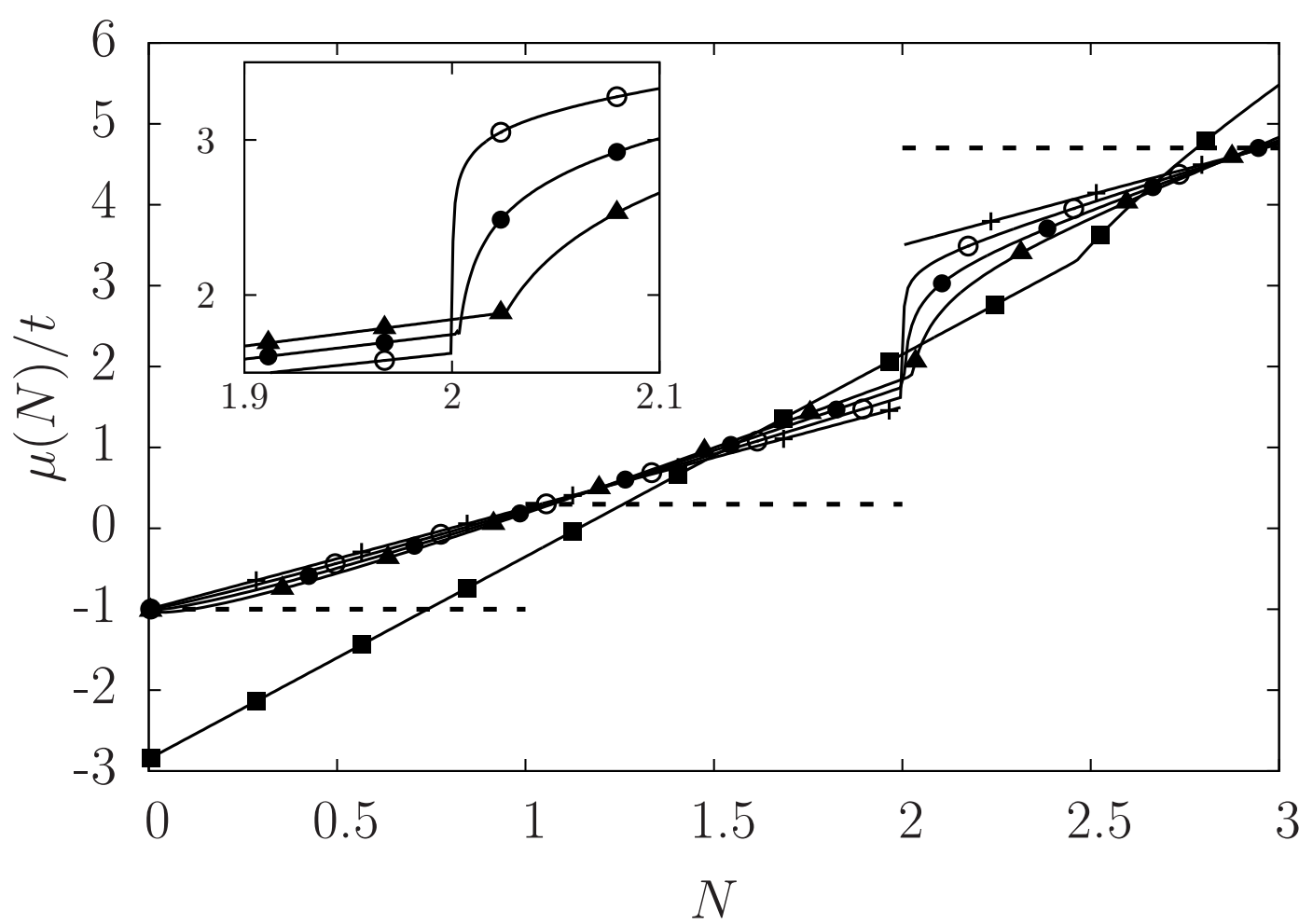

Figure 4.13: Chemical potential $\mu(N)$ of the Hubbard dimer with $U=5 t$ in units of the hopping parameter $t$ as function of particle number $N$, when the density matrix is restricted to be non-magnetic. The behavior of the power functional with $1 / 2<\alpha<1$ close to half-filling is shown in the inset. Dashed line: exact solution, crosses: Hartree-Fock approximation, open circles: power functional with $\alpha=0.95$, filled circles: power functional with $\alpha=0.9$, triangles: power functional with $\alpha=0.85$, squares: Müller functional.

The Müller functional [50] establishes left-right correlation in a fundamentally different manner: while the natural orbitals are mostly - in the Hubbard dimer exactlyindependent of the interaction, the occupations become fractional, which reflects the creation of electron-hole pairs that screen the interaction. One of the main successes of the Müller functional besides being able to produce fractional occupations correctly, is that it captures the continuous nature of the transition to the left-right-correlated state.

Our calculations avoid any bias and allow for arbitrary non-collinear spin-polarized states. This strategy shall bring all potential problems to the surface, that would be present in large scale electronic structure calculations using these density-matrix functionals.

Our first observation is that the ground state for the Müller functional, which does 


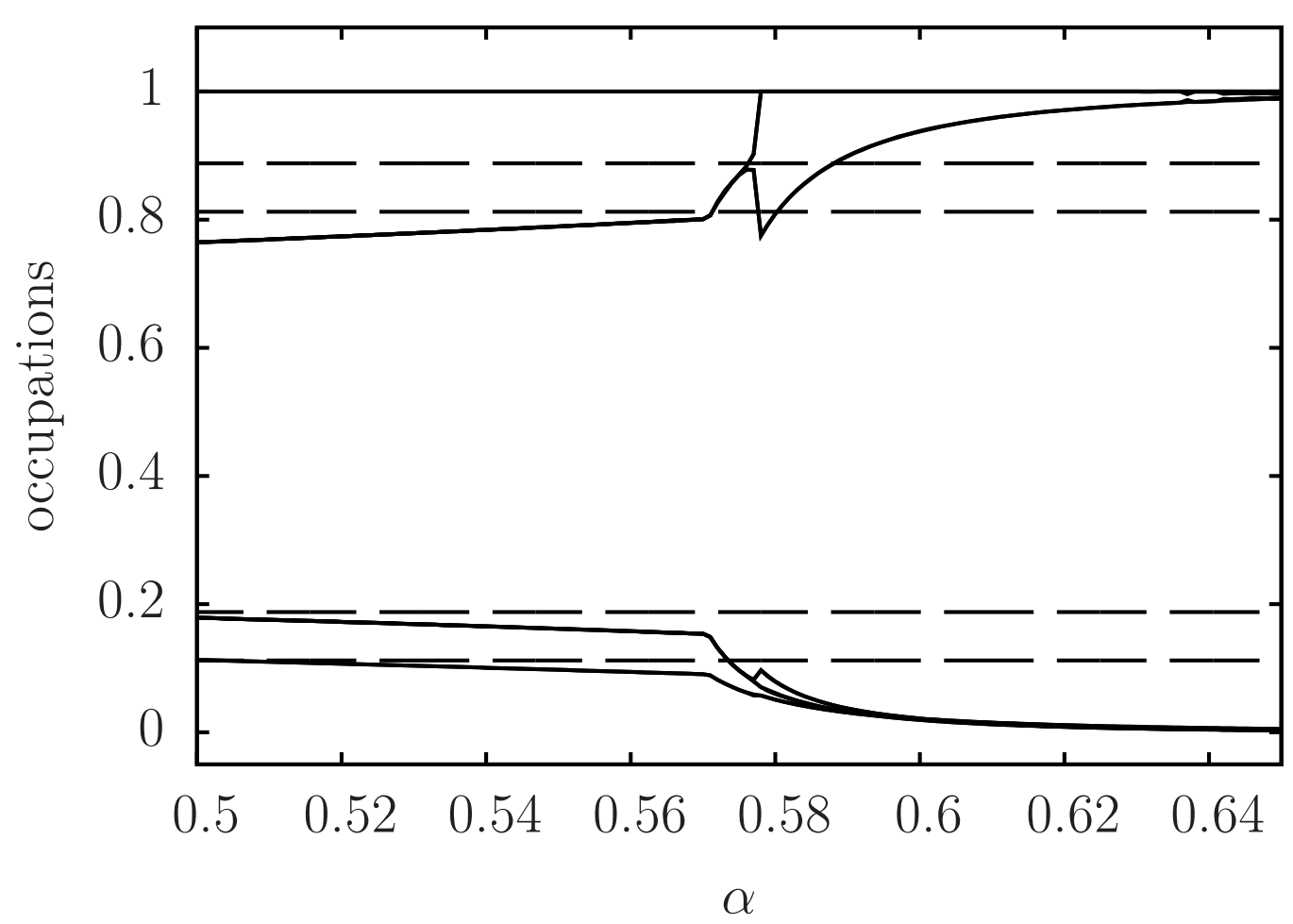

Figure 4.14: Occupations of the half-filled six-site Hubbard-ring with $U=5 t$ for the power functional as function of the parameter $\alpha$ (solid lines). The dashed horizontal lines indicate the occupations of the exact many-electron description. Evident are the rather abrupt transitions from fractional to integer occupations.

not have local moments, is degenerate with a one dimensional manifold of ferromagnetic states. Thus the dimer has infinite magnetic susceptibility when described with the Müller functional, in contrast to the vanishing zero-temperature susceptibility of the exact solution of the Hubbard dimer. This large magnetic polarizability is likely to cause severe problems in extended electronic structure calculations.

When turning to the power functional[53], we find that the system behaves analogous to the Müller functional for small interactions, while it exhibits a transition to a Hartree-Fock-like antiferromagnetic state for large interactions. The critical interaction, where this transition occurs, drops rapidly with increasing $\alpha$ from infinity in the Müller functional to the Hartree-Fock value $U_{\text {crit }}=2 t$.

In the small-interaction regime the system is weakly pinned in the ferromagnetic state corresponding to the largest moment of the ground-state manifold of the Müller functional.

Our calculations indicate a major deficiency in the description of magnetic properties for this class of density-matrix functionals. The problems persist in modified 


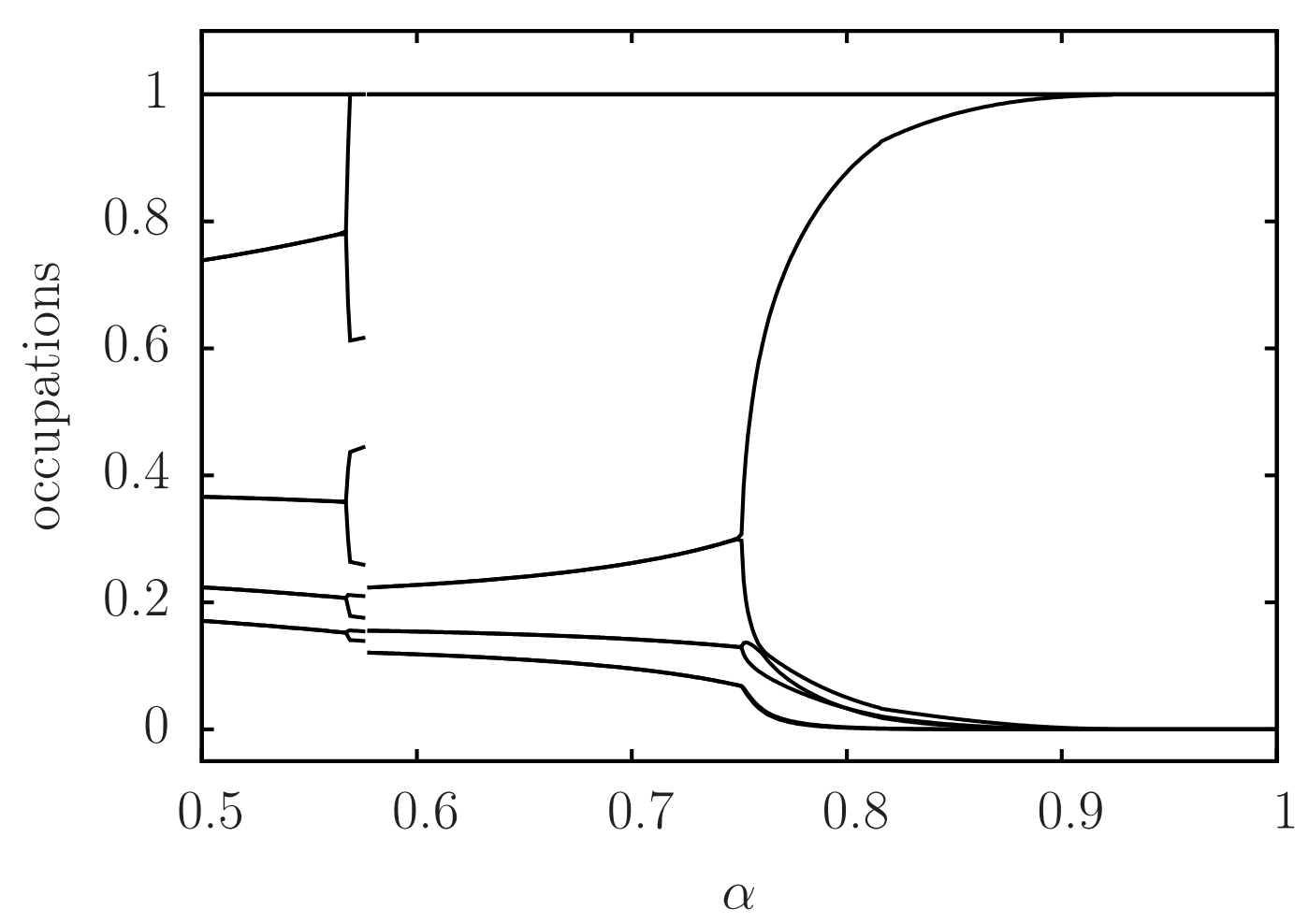

Figure 4.15: Occupations of the six-site Hubbard-chain with seven electrons and $U=5 t$ for ground states of the power functional as function of the parameter $\alpha$ spanning the range from the Müller functional $(\alpha=1 / 2)$ to the HartreeFock approximation $(\alpha=1)$. Evident are the rather abrupt transitions from fractional to integer occupations.

form also for more general Hamiltonians, which include off-site Coulomb interactions, and for more extended systems.

Besides the bond-dissociation problem, we investigated the derivative discontinuity 94 , 28 with changing the number of electrons. A balanced description of the electron affinity and ionization potential is essential for a qualitatively correct description of charge transfer. We find that the metal-like behavior of the Müller functional persists: The discontinuity of the exchange-correlation energy even offsets the one of the kinetic energy. The Müller functional describes the Hubbard dimer with vanishing fundamental gap.

The power functional inherits many of the problems of the Müller functional: There is no derivative discontinuity in the entire parameter range of the power functional except for the Hartree-Fock limit. In the low-interaction regime the solutions are weakly ferromagnetic. Like the Hartree-Fock approximation, the power functional exhibits an artificial abrupt magnetic transition with increasing interaction 
towards an antiferromagnetic configuration, albeit at a larger critical interaction. These states are intrinsically non-collinear.

The absence of any derivative discontinuity also for insulating materials is expected to produce an artificial charge transfer between the constituents of large-scale electronic structure calculations. This cast severe doubt on the performance of such density-matrix functionals for complex systems.

While the power functional lacks a derivative discontinuity, its chemical potential undergoes a continuous transition between two linear functions, which has been exploited to extract a band gap from data obtained further away from the integer particle number [53, 91, 95, 89, 90].

Our calculations indicate, however, that the band gap obtained from this extrapolation can be tuned by the free parameter $\alpha$ of the power functional between zero and the Hartree-Fock result. The band gap opens in non-collinear calculations only when in the antiferromagnetic regime, while it vanishes in the Müller-type regime at low interactions. The opening of a band gap obtained by the extrapolation method and its tunability are features that persist in non-magnetic calculations, while the gap opens at a larger value of the power parameter than in the magnetic calculation. These problems or signatures of them can be observed in previous calculations [95, 90, 80, 93, 92].

The problems presented here demonstrate potential fundamental flaws of the class of density-matrix functionals of this study. 


\section{Density matrix functionals derived from Green's function}

In Chapter. 4, we demonstrated the fundamental flaws in the existing state of the art density matrix functionals namely the parametrized density matrix functionals of Sec. 2.6.3. The most prominent among all, the celebrated Müller class of functionals had serious drawbacks in determining the magnetic structures of even simple model systems. The quality of such functionals depends extensively on parameters which cannot be determined in a controled way.

In Sec. 2.6.2, we briefly discussed the way to evaluate density matrix functional from the Green's function functional namely the Luttinger-Ward functional. The exact relation [48] between the two requires a stationary point search in an exponential space of the Green's function and the self-energy. But nevertheless, it allows to construct approximations in a controlled way via immensely studied techniques of perturbation theory in the many-body community.

In this chapter, we propose several approximations to evaluate density matrix functional from the Green's function and assess their quality by comparing with the performance of existing state of the art density matrix functionals. The simple model system of Sec. 2.7, namely the Hubbard dimer will be used as a benchmark system to test the quality of the proposed approximations.

\subsection{Framework for construction of approximate scheme}

In Sec. 2.6.2, we presented the exact relation between the density matrix functional and the Luttinger-Ward functional. The exact expression has the following form,

$$
\begin{aligned}
F^{\hat{\boldsymbol{W}}}[\boldsymbol{\rho}]= & \frac{1}{\beta} \operatorname{Tr}[\boldsymbol{\rho} \ln (\boldsymbol{\rho})+(\mathbf{1}-\boldsymbol{\rho}) \ln (\mathbf{1}-\boldsymbol{\rho})] \\
+ & \operatorname{stat}_{\boldsymbol{h}^{\prime}} \operatorname{stat}_{\boldsymbol{G}, \boldsymbol{\Sigma}}\left\{\Phi_{\beta}^{\hat{W}}[\boldsymbol{G}, \hat{W}]-\frac{1}{\beta} \sum_{\nu} \operatorname{Tr}\left\{\ln \left[\mathbf{1}-\overline{\boldsymbol{G}}\left(i \omega_{\nu}\right)\left(\boldsymbol{h}^{\prime}+\boldsymbol{\Sigma}\left(i \omega_{\nu}\right)-\overline{\boldsymbol{h}}\right)\right]\right.\right. \\
& \left.\left.+\left(\boldsymbol{h}^{\prime}+\boldsymbol{\Sigma}\left(i \omega_{\nu}\right)-\overline{\boldsymbol{h}}\right) \boldsymbol{G}\left(i \omega_{\nu}\right)-\left[\boldsymbol{G}\left(i \omega_{\nu}\right)-\overline{\boldsymbol{G}}\left(i \omega_{\nu}\right)\right]\left(\boldsymbol{h}^{\prime}-\overline{\boldsymbol{h}}\right)\right\}\right\}
\end{aligned}
$$


where $\Phi_{\beta}^{\hat{W}}[\boldsymbol{G}, \hat{W}]$ is the Luttinger-Ward functional [49, 70], $\overline{\boldsymbol{G}}\left(i \omega_{\nu}\right)=\left(\left(i \omega_{\nu}+\mu\right) \mathbf{1}-\right.$ $\overline{\boldsymbol{h}})^{-1}$ is the non-interacting Green's function which is a direct functional of $\boldsymbol{\rho}$ through $\overline{\boldsymbol{h}}=\mu-\frac{1}{\beta} \ln \left(\frac{\mathbf{1 - \rho}}{\boldsymbol{\rho}}\right)$. The Lagrange multiplier $\boldsymbol{h}^{\prime}$ enforces the density matrix constraint,

$$
\boldsymbol{\rho}[\boldsymbol{G}]=\frac{1}{\beta} \sum_{\omega_{\nu}} \mathrm{e}^{i \omega_{\nu} 0^{+}} \boldsymbol{G}\left(i \omega_{\nu}\right)
$$

The non-interacting Green's function $\overline{\boldsymbol{G}}\left(i \omega_{\nu}\right)$, by construction satisfies the density matrix constraint of Eq. (5.2).

As evident from Eq. 5.1 , the evaluation of density matrix functional $F_{\beta}^{\hat{W}}[\boldsymbol{\rho}]$ requires an internal optimization in the space of the Green's function $\boldsymbol{G}\left(i \omega_{n}\right)$ and the corresponding self energy $\boldsymbol{\Sigma}\left(i \omega_{n}\right)$ with an additional complexity of the density matrix constrained of Eq. (5.2). The internal optimization requires solution for a set of self-consistent equations [48].

\section{Approximate scheme}

The most desirable approximate scheme will be the one which completely avoids the stationary point search in Eq. (5.1), but nevertheless describes the essential physics. In order to bypass the full internal optimization, we define a mapping of the form,

$$
\rho \rightarrow G[\rho]
$$

which provides a Green's function $\boldsymbol{G}(\boldsymbol{\rho})$ for a given one-particle density matrix $\boldsymbol{\rho}$. The Green's function obtained from the mapping in Eq. 5.3), by construction satisfies the density matrix constraint of Eq.5.2.

The mapping also helps to avoid the stationary point search in Eq.(5.1) and leads to an error $\Delta F_{\beta}^{\hat{W}}[\boldsymbol{\rho}]$ in the calculations of density matrix functional. It can be shown [48, that the error $\Delta F_{\beta}^{\hat{W}}[\boldsymbol{\rho}]$ is quadratic in the variation of the model Green's function $\boldsymbol{G}[\rho]$ from true Green's function $\boldsymbol{G}_{\text {true }}$,

$$
\begin{aligned}
\Delta F_{\beta}^{\hat{W}}[\boldsymbol{\rho}] & =\Phi_{\beta}^{L W}\left[\boldsymbol{G}_{\text {true }}, \hat{W}\right]-\Phi_{\beta}^{L W}[\boldsymbol{G}[\boldsymbol{\rho}], \hat{W}] \\
& +\sum_{n} \operatorname{Tr}\left\{\frac{\partial \Phi_{\beta}^{L W}}{\partial \boldsymbol{G}\left(i \omega_{n}\right)}\left(\boldsymbol{G}[\rho]\left(i \omega_{n}\right)-\boldsymbol{G}\left(i \omega_{n}\right)\right)\right\} \\
& +\frac{1}{\beta} \sum_{n} \operatorname{Tr}\left\{\sum_{m=2}^{\infty} \frac{1}{m}\left(1-\boldsymbol{G}[\boldsymbol{\rho}]\left(i \omega_{n}\right) \boldsymbol{G}^{-1}\left(i \omega_{n}\right)\right)^{m}\right\}
\end{aligned}
$$

With the direct mapping suggested in Eq.(5.3), the stationarity condition with 
respect to the self-energy leads to the Dyson equation of the form,

$$
\Sigma\left(i \omega_{n}\right)=\overline{\boldsymbol{h}}+\overline{\boldsymbol{G}}^{-1}\left(i \omega_{n}\right)-\boldsymbol{G}^{-1}\left(i \omega_{n}\right)-\boldsymbol{h}^{\prime}
$$

Substituting this form of the stationary self-energy in Eq. (5.1), and using the fact that $\boldsymbol{G}[\boldsymbol{\rho}]$ satisfies the density matrix constraint which leads to dropping off the last part in Eq (5.1), the density matrix functional $F_{\beta}^{\hat{W}}[\boldsymbol{\rho}]$ attains a simple algebraic form,

$$
\begin{aligned}
F_{\beta}^{\hat{W}}[\boldsymbol{\rho}] & \approx \frac{1}{\beta} \operatorname{Tr}[\boldsymbol{\rho} \ln (\boldsymbol{\rho})+(\mathbf{1}-\boldsymbol{\rho}) \ln (\mathbf{1}-\boldsymbol{\rho})]+\Phi_{\beta}^{L W}[\boldsymbol{G}[\boldsymbol{\rho}], \hat{W}] \\
& -\frac{1}{\beta} \sum_{\omega_{n}} \operatorname{Tr}\left\{\ln \left[\overline{\boldsymbol{G}}\left(i \omega_{n}\right) \boldsymbol{G}^{-1}\left(i \omega_{n}\right)\right]+\overline{\boldsymbol{G}}^{-1}\left(i \omega_{n}\right) \boldsymbol{G}\left(i \omega_{n}\right)-\mathbf{1}\right\}
\end{aligned}
$$

The simple mapping in Eq. (5.3), leads to the direct evaluation of density matrix functional from the Luttinger-Ward functional evaluated for the model Green's function $\boldsymbol{G}[\boldsymbol{\rho}]$. Further approximation comes in the evaluation of Luttinger-Ward functional which is done perturbatively by expanding in the powers of interaction strength $U$. This is discussed in the next section.

\subsection{Approximation for Luttinger Ward functional $\Phi_{\beta}^{L W}[\boldsymbol{G}[\boldsymbol{\rho}], \hat{W}]$}

The Luttinger-Ward functional [49, 70] is defined through a perturbative expansion in the interaction strength. It is obtained by summing up an infinite set of skeleton diagrams with no self energy insertions.

The density matrix functional as stated earlier is evaluated in a controlled way by considering the diagrammatic expansion of the Luttinger-Ward functional for a given model Green's function $\boldsymbol{G}[\boldsymbol{\rho}]$.

\subsubsection{Hartree-Fock approximation}

The first order skeleton diagrams in the perturbation expansion are the so called Hartree-Fock diagrams as shown in the Fig. 5.1. The approximate Luttinger-Ward functional $\Phi_{\beta}^{H F}[\boldsymbol{G}[\boldsymbol{\rho}], \hat{W}]$ corresponding to the Hartree-Fock approximation is given in terms of the two diagrams $D_{a}$ and $D_{b}$ in Fig. 5.1 as,

$$
\Phi_{\beta}^{\mathrm{HF}}[\boldsymbol{G}[\boldsymbol{\rho}], \hat{W}]=-\frac{1}{\beta} \sum_{D=D_{a}, D_{b}} \frac{V(D)}{S(D)}
$$


The value of the diagrams $V(D)$ is evaluated using the diagrammatic rules sketched in Appendix C. The number of closed Fermionic loops for Hartree diagram is $n_{f}=2$ whereas for the Fock diagram is $n_{f}=1$.

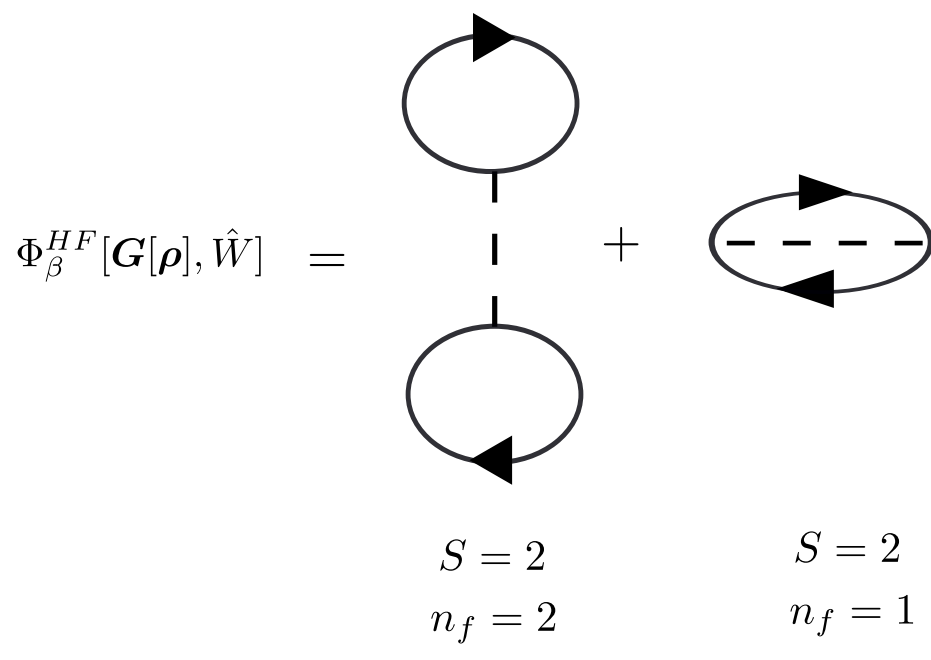

(a)

(b)

Figure 5.1: The skeleton diagrams contributing to the first order in the perturbation expansion of Luttinger Ward functional. The symmetry factor for the Hartree diagram (a) is $S=2$ and the number of fermi loops is $n_{f}=2$. The symmetry factor for the Fock diagram (b) is $S=2$ and the number of fermi loops is $n_{f}=1$.

The complete algebraic expression for the Hartree-Fock contribution has the form,

$$
\begin{aligned}
\Phi_{\beta}^{H F}[\boldsymbol{G}[\boldsymbol{\rho}], \hat{W}] & =\frac{1}{2} \sum_{a b, d c} W_{a b, c d} \frac{1}{\beta^{2}} \sum_{\omega_{n_{1}} \omega_{n_{2}}}\left(\boldsymbol{G}_{d a}\left(i \omega_{n_{1}}\right) \boldsymbol{G}_{c b}\left(i \omega_{n_{2}}\right)-\boldsymbol{G}_{c a}\left(i \omega_{n_{1}}\right) \boldsymbol{G}_{d b}\left(i \omega_{n_{2}}\right)\right) \\
& =\frac{1}{2} \sum_{a b, c d} W_{a b, d c}\left(\boldsymbol{\rho}_{d a} \boldsymbol{\rho}_{c b}-\boldsymbol{\rho}_{c a} \boldsymbol{\rho}_{d b}\right)
\end{aligned}
$$

where $\omega_{n}=\frac{(2 n+1) \pi}{\beta}$ is the fermionic Matsubara frequency and we have used the fact that $\frac{1}{\beta} \sum_{i \omega_{n}} \boldsymbol{G}\left(i \omega_{n}\right)=\boldsymbol{\rho}$.

It is evident from Eq. (5.8), the Luttinger Ward functional in the first order is a direct functional of one particle reduced density matrix $\boldsymbol{\rho}$ and does not depend on the choice of the model Green's function. 


\subsubsection{Second order diagrams}

Beyond the Hartree-Fock approximation, the diagrams which include non-trivial correlation effects are the second order diagrams. In the second order of perturbation in the interaction, there are two skeleton diagrams as shown in Fig. 5.2, The first diagram is the bubble or the "tad-pole" diagram whereas the second is the exchange contribution. The value of Luttinger-Ward function $\Phi_{\beta}^{(2)}[\boldsymbol{G}[\boldsymbol{\rho}], \hat{W}]$ in the second

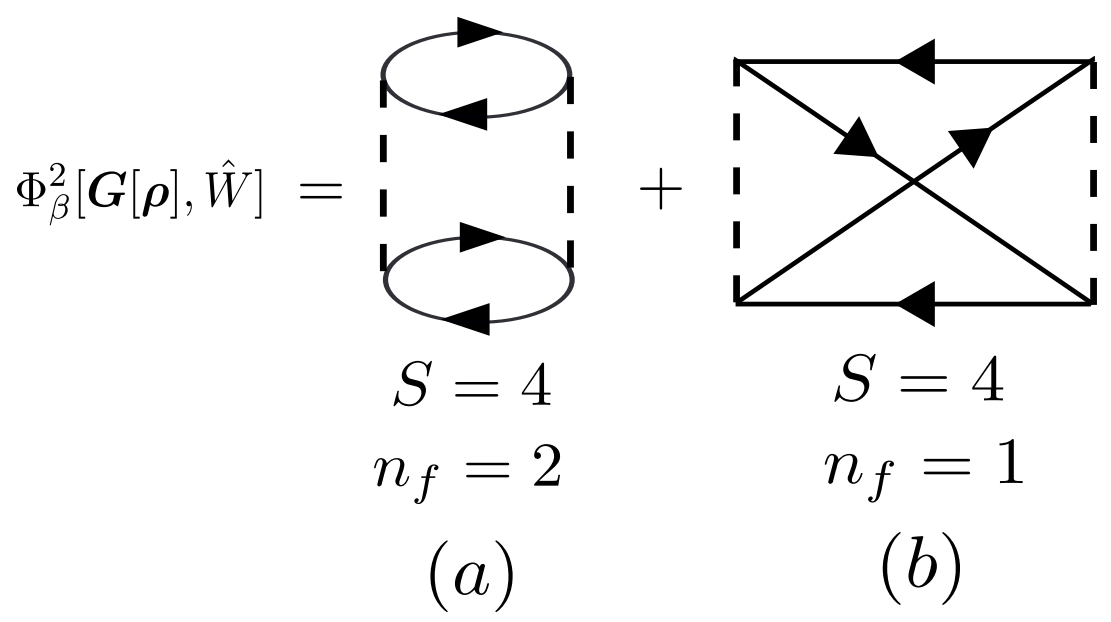

Figure 5.2: The skeleton diagrams contributing to the second order in the perturbation expansion of Luttinger Ward functional. The symmetry factor for the bubble diagram (a) is $S=4$ and the number of Fermi loops is $n_{f}=2$. The symmetry factor for the exchange diagram (b) is $S=4$ and the number of Fermi loops is $n_{f}=1$.

order of the perturbation expansion can be written as a sum of two diagrams (See. Eq. (C.16) in Sec C.4 as,

$$
\Phi_{\beta}^{(2)}[\boldsymbol{G}[\boldsymbol{\rho}], \hat{W}]=-\frac{1}{\beta} \sum_{D=D_{a}, D_{b}} \frac{V(D)}{S(D)}
$$

The value of the diagrams are evaluated according to the rules given in Sec. C. The symmetry factors of both the diagrams is 4 . The value of the second order diagrams depends on the choice of the model Green's function. Both diagrams depends on a second rank tensorial object called the polarization $\chi$, which has the form,

$$
\chi_{a b, c d}\left(i \nu_{n}\right)=\frac{1}{\beta} \sum_{\omega_{n}} \boldsymbol{G}_{d a}\left(i \omega_{n}+i \nu_{n}\right) \boldsymbol{G}_{c b}\left(i \omega_{n}\right)
$$


where the frequency $\nu_{n}=\frac{2 n \pi}{\beta}$ is the bosonic Matsubara frequency. The fermionic Matsubara sum in Eq. 5.10) can be performed analytically using the multi-pole representation of the Green's function.

Using the polarization tensor $\chi\left(\nu_{n}\right)$ it can be shown easily that the combined mathematical expression for the two second order diagrams in Fig. 5.2 has the following form,

$$
\begin{aligned}
\Phi_{\beta}^{2}[\boldsymbol{G}[\boldsymbol{\rho}], \hat{W}] & =-\frac{1}{4 \beta} \sum_{\nu_{n}}\left(\operatorname{Tr} \boldsymbol{Y}_{\text {bubble }}^{2}\left(i \nu_{n}\right)-\operatorname{Tr} \boldsymbol{Y}_{\mathrm{exch}}^{2}\left(i \nu_{n}\right)\right) \\
\boldsymbol{Y}_{a b, c d}^{\text {bubble }}\left(i \nu_{n}\right) & =\sum_{e f} W_{e a, f b} \boldsymbol{\chi}_{f d, e c}\left(i \nu_{n}\right) \\
\boldsymbol{Y}_{a b, c d}^{\text {exch. }}\left(i \nu_{n}\right) & =\sum_{e f} W_{e a, b f} \boldsymbol{\chi}_{f d, e c}\left(i \nu_{n}\right)
\end{aligned}
$$

As evident from the expression in Eq. (5.11), the objects $\boldsymbol{Y}^{\text {bubble }}$ and $\boldsymbol{Y}^{\text {exch. }}$ corresponding to the bubble and exchange diagrams respectively, can be obtained from each other by interchanging the last two indices of the interaction vertex. The negative sign in the exchange diagram comes from the fact the the number of closed Fermi loops in diagram (b) is $n_{f}=1$.

\section{Hugenholtz diagrams}

It is worthwhile to illustrate at this stage, the importance of Hugenholtz diagrams [9] which drastically reduces the number of diagrammatic evaluations at higher orders. The Hugenholtz diagram are obtained by replacing the conventional interaction vertex with an anti-symmetrized vertex as,

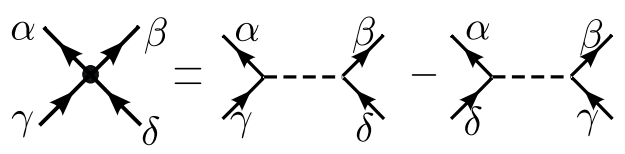

Below we state few important points that need to be taken care of while evaluating the Luttinger-Ward functional in terms of the Hugenholtz diagrams,

1. The value $V(D)$ of the Hugenholtz diagrams are also evaluated using the rules prescribed in Appendix C, with a slight modification that for each interaction vertex we include the factor,

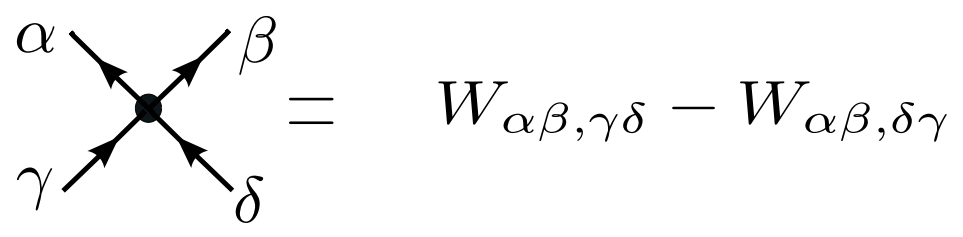


2. During the evaluation of value $V(D)$, the number of fermion loops $n_{f}$ are evaluated by replacing the Hugenholtz vertex by the conventional vertex and counting the number of closed fermion loops as for a Feynman diagram.

3. The mathematical expression for the Luttinger-Ward functional in terms of the Hugenholtz diagram has the form,

$$
\Phi_{\beta}^{2}[\boldsymbol{G}[\boldsymbol{\rho}], \hat{W}]=-\frac{1}{\beta} \sum_{D} \frac{V(D)}{2^{n_{e}(D)} S(D)}
$$

where $n_{e}(D)$ is the number of equivalent pair of lines i.e. the number of pairs of lines originating at the same vertex, terminating at the same vertex and oriented in the same direction for a given diagram $D$.

The two skeleton diagrams each in the first (See Fig. 5.1) and second-order (See Fig. 5.2) of the perturbation expansion can be obtained from one Hugenholtz diagram each respectively as,

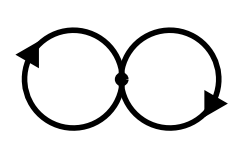

$$
\begin{gathered}
S=1 \\
n_{e}=1 \\
n_{f}=2 \\
a
\end{gathered}
$$

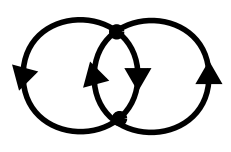

$S=2$
$n_{e}=2$

$n_{f}=2$

Figure 5.3: The Hugenholtz skeleton diagrams equivalent to the (a) Hartree-Fock and (b) the second order "tad-pole" and the corresponding exchange diagram.

\section{Example diagram}

Using the fact that the symmetry factor for the second order Hugenholtz diagram in Fig. 5.3(b) is $S=2$, the number of fermion loops is $n_{f}=2$ and the number of equivalent pair of lines is $n_{e}=2$, the value of the second order approximation to the Luttinger-Ward functional has the form,

$$
\begin{aligned}
\Phi_{\beta}^{2}[\boldsymbol{G}[\boldsymbol{\rho}], \hat{W}] & =-\frac{1}{8 \beta} \sum_{\nu_{n}} \operatorname{Tr}\left\{\left(\boldsymbol{Y}^{\mathrm{RPA}-\mathrm{PH}}\left(i \nu_{n}\right)\right)^{2}\right\} \\
\boldsymbol{Y}_{a b, c d}^{\mathrm{RPA}-\mathrm{PH}}\left(i \nu_{n}\right) & =\sum_{e f}\left(W_{e a, f b}-W_{e a, b f}\right) \chi_{f d, e c}\left(i \nu_{n}\right)
\end{aligned}
$$

The expression in Eq. (5.13) when expanded, is equal to the combined contribution of the "tad-pole" and the corresponding exchange diagram in Fig. 5.2. The extra factor of 2 in Eq. (5.13) takes care of the over-counting each diagram twice. 


\subsubsection{Higher order diagrams}

The systematic evaluation of higher order diagrams in the weak-coupling limit (low $\mathrm{U})$ is computationally demanding and is not the best way to approximate the Luttinger-Ward functional. The most feasible way to move beyond second order diagrams, is to look for a class of diagrams which can be summed up analytically to infinite orders. Inclusion of only a set of diagrams in the expansion of LuttingerWard functional might lead to unphysical results due to violation of conservation laws [96]. For example in the case of spin dependent interactions, at the second order of perturbation, one needs to consider both skeleton diagrams of Fig. 5.2. Ignoring the exchange diagram (b) in Fig. 5.2 will lead to violation of Pauli exclusion principle [97]. In 1989, Bickers and Scalapino [98, 99, 100] proposed fluctuation-exchange

$$
\Phi_{\beta}^{\mathrm{RPA} P \mathrm{PH}}[\boldsymbol{G}[\boldsymbol{\rho}], \hat{W}]=
$$

Figure 5.4: The Hugenholtz skeleton diagrams contributing to the particle-hole channel in random phase approximation (RPA) of the Luttinger Ward functional. For the diagram with $n$ interaction vertices, the symmetry factor is $S(D)=2 n$, the number of closed fermi loops is $n_{f}=n$ and the number of equivalent pair of line is $n_{e}=0$.

(FLEX) approximation which originated from the conserving approximation theory proposed by Baym and Kadanoff [96]. The FLEX approximation is the simplest infinite order conserving approximation which treats the collective particle-hole and particle-particle excitations. It has been widely applied to single and multi-orbital Hubbard model [98, 99], in which the spin rotational invariance is respected.

In case of the general interaction tensor which allows for the breaking of spin rotational invariance, the infinite series of closed skeleton diagrams of LuttingerWard functional within random-phase-approximation (RPA) in Fig. 5.4 and Fig. 5.5 form the body of FLEX diagrams [101]. The Hugenholtz diagrams were found to be more suited to deal with the $\mathrm{SU}(2)$ broken case [101].

The infinite series of the particle-hole channel within RPA (See Fig. 5.4) can be summed up analytically to obtain a closed expression for the random-phase- 
approximation for the Luttinger-Ward functional $\Phi_{\beta}^{\mathrm{RPA}-\mathrm{PH}}[\boldsymbol{G}[\boldsymbol{\rho}], \hat{W}]$ given by,

$$
\Phi_{\beta}^{\mathrm{RPA}-\mathrm{PH}}[\boldsymbol{G}[\boldsymbol{\rho}], \hat{W}]=-\frac{1}{\beta} \sum_{D \in \mathrm{RPA}-\mathrm{PH}} \frac{V(D)}{2^{n_{e}(D)} S(D)}
$$

For a diagram $D$ in the RPA-PH series with $n>2$ Hugenholtz interaction vertices (anti-symmetrized vertex), the symmetry factor is $S(D)=2 n$ and the number of closed fermion loops are $n_{f}=n$. Therefore the sign of each diagram with $n$ vertices is $(-1)^{n+n_{f}}=1$. The number of equivalent pair of lines $n_{e}(D)=0$.

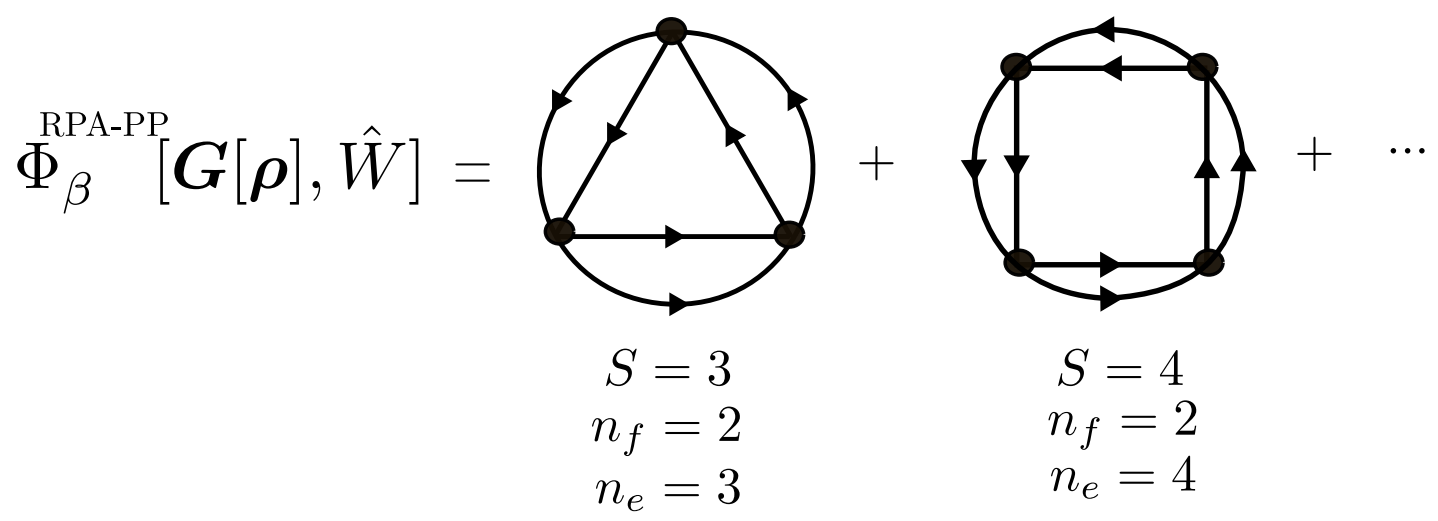

Figure 5.5: The Hugenholtz skeleton diagrams contributing to the particle-particle channel in random phase approximation (RPA) of the Luttinger Ward functional. For the diagram with $n$ interaction vertices, the symmetry factor is $S(D)=n$, the number of closed fermi loops is $n_{f}=2$ and the number of equivalent pair of line is $n_{e}=n$.

The building block of each diagram is the tensor $\boldsymbol{Y}^{\mathrm{RPA}-\mathrm{PH}}\left(i \nu_{n}\right)$, the expression for which is given in Eq. (5.13). The series can be summed up analytically and has the following form,

$$
\begin{aligned}
\Phi_{\beta}^{\mathrm{RPA}-\mathrm{PH}}[\boldsymbol{G}[\boldsymbol{\rho}], \hat{W}] & =-\frac{1}{\beta} \sum_{n=3}^{\infty} \frac{1}{2 n} \sum_{\nu_{n}} \operatorname{Tr}\left(\boldsymbol{Y}^{\mathrm{RPA}-\mathrm{PH}}\left(i \nu_{n}\right)\right)^{n} \\
& =\frac{1}{2 \beta} \sum_{\nu_{n}} \operatorname{Tr}\left\{\ln \left(\mathbf{1}-\boldsymbol{Y}^{\mathrm{RPA}-\mathrm{PH}}\left(i \nu_{n}\right)\right)+\boldsymbol{Y}^{\mathrm{RPA}-\mathrm{PH}}\left(i \nu_{n}\right)\right. \\
& \left.+\frac{1}{2}\left(\boldsymbol{Y}^{\mathrm{RPA}-\mathrm{PH}}\left(i \nu_{n}\right)\right)^{2}\right\}
\end{aligned}
$$

The particle-particle channel within the RPA series in Fig. 5.5 is also referred to as the T-matrix approximation (TMA) proposed by V. M. Galitskii [102] in 1958. 
The TMA was proposed in the context of non-ideal Fermi gas where the interaction between particles is short range i.e. inter-particle distance is much larger than the range of interaction.

For a diagram $D$ in the RPA-PP series with $n>2$ Hugenholtz interaction vertices (anti-symmetrized vertex), the symmetry factor is $S(D)=n$ and the number of closed fermion loops are $n_{f}=2$. The number of equivalent pair of lines $n_{e}(D)=n$.

The contribution of the particle-particle channel of the RPA series to the LuttingerWard functional is denoted as $\Phi_{\beta}^{\mathrm{RPA}-\mathrm{PP}}[\boldsymbol{G}[\boldsymbol{\rho}], \hat{W}]$ and the building block $\boldsymbol{Y}^{\mathrm{RPA}-\mathrm{PP}}\left(i \nu_{n}\right)$ is used for the evaluation of the value of each diagram $V(D)$. These quantities have the following form,

$$
\begin{aligned}
\Phi_{\beta}^{\mathrm{RPA}-\mathrm{PP}}[\boldsymbol{G}[\boldsymbol{\rho}], \hat{W}] & =-\frac{1}{\beta} \sum_{n=3}^{\infty} \frac{1}{n 2^{n}} \sum_{\nu_{n}} \operatorname{Tr}\left(\boldsymbol{Y}^{\mathrm{RPA}-\mathrm{PP}}\left(i \nu_{n}\right)\right)^{n} \\
& =-\frac{1}{\beta} \sum_{\nu_{n}} \operatorname{Tr}\left\{\ln \left(\mathbf{1}-\frac{\boldsymbol{Y}^{\mathrm{RPA}-\mathrm{PP}}\left(i \nu_{n}\right)}{2}\right)+\frac{\boldsymbol{Y}^{\mathrm{PP}}\left(i \nu_{n}\right)}{2}\right. \\
& \left.+\frac{1}{2}\left(\frac{\boldsymbol{Y}^{\mathrm{RPA}-\mathrm{PP}}\left(i \nu_{n}\right)}{2}\right)^{2}\right\} \\
Y_{a b, c d}^{\mathrm{RPA}-\mathrm{PP}}\left(i \nu_{n}\right) & =-\sum_{e f}\left(W_{a b, e f}-W_{a b, f e}\right) \frac{1}{\beta} \sum_{\omega_{n}} G_{c e}\left(i \nu_{n}-i \omega_{n}\right) G_{d f}\left(i \omega_{n}\right)
\end{aligned}
$$

\subsubsection{Regularization}

As shown in the previous section, the perturbation expansion of Luttinger Ward functional requires summation over Matsubara frequencies which needs to be performed numerically.

The algebraic expressions for each diagram or certain class of diagrams in the perturbative expansion of Luttinger-Ward functional has the following general form,

$$
\mathcal{S}=\frac{1}{\beta} \sum_{i \omega_{n}} \mathcal{F}\left(i \omega_{n}\right)
$$

where $\omega_{n}$ can be bosonic or fermionic Matsubara frequencies. In principle, one needs to perform this sum over infinite number of discrete Matsubara frequencies. However, in practice, we perform a frequency cut-off where we restrict the sum to some large frequency $\Omega$.

It is observed that at low temperatures the value of $\mathcal{F}\left(i \omega_{n}\right)$ converges slowly with increasing Matsubara frequencies. Therefore, at low temperatures, the sum in Eq. 5.17) require large number of frequencies to be included. This in turn slows down the numerical simulations. 
Therefore, we need to come up with the scheme where we can evaluate the behavior of the tail analytically. This requires to evaluate the behavior of $\mathcal{F}\left(i \omega_{n}\right)$ at large frequencies which can be obtained using Laurent expansion in $\omega_{n}$ as,

$$
\mathcal{F}_{\text {tail }}\left(i \omega_{n}\right)=\sum_{j} \frac{C_{j}}{\left(i \omega_{n}\right)^{j}}
$$

Using the behavior of the tail, we can approximate the sum in Eq.5.17) as,

$$
\mathcal{S} \approx \frac{1}{\beta}(\sum_{i \omega_{n}=-\Omega}^{\Omega} \mathcal{F}\left(i \omega_{n}\right)+\underbrace{\sum_{j} C_{j} \sum_{i \omega_{n}=-\infty}^{\infty} \frac{1}{\left(i \omega_{n}\right)^{j}}-\sum_{j} C_{j} \sum_{i \omega_{n}=-\Omega}^{\Omega} \frac{1}{\left(i \omega_{n}\right)^{j}}}_{\text {Tail correction }})
$$

In this method of regularization we evaluate the behavior of the tail analytically using the following formula [8],

$$
\frac{1}{\beta} \sum_{i \omega_{n}=-\infty}^{\infty} \frac{1}{\left(i \omega_{n}-\zeta\right)^{j}}=-\frac{\eta}{(j-1) !} \partial_{\zeta}^{j-1} n_{\eta}(\zeta)
$$

where $\eta= \pm 1$ for bosons and fermions respectively and

$$
n_{\eta}(\zeta)= \begin{cases}\frac{1}{e^{\beta \zeta}-1}, & \text { for } \eta=1 \\ \frac{1}{e^{\beta \zeta}+1}, & \text { for } \eta=-1\end{cases}
$$

The procedure in Eq. (5.19), leads to an exact evaluation of the sum at large frequencies due to the availability of analytical expressions of the form in Eq. (5.20).

NOTE: An extra precaution has to be taken while performing the sum over bosonic matsubara frequencies in the tail correction part of Eq. 5.19) because it requires the correction to be evaluated for $\omega_{n}=0$. The correction term thus assume the following form,

$$
\sum_{j} C_{j} \sum_{\substack{i \omega_{n}=-\infty \\ \omega_{n} \neq 0}}^{\infty} \frac{1}{\left(i \omega_{n}\right)^{j}}-\sum_{j} C_{j} \sum_{\substack{i \omega_{n}=-\Omega \\ \omega_{n} \neq 0}}^{\Omega} \frac{1}{\left(i \omega_{n}\right)^{j}}
$$

where the infinite sum can still be evaluated analytically as,

$$
\frac{1}{\beta} \sum_{\substack{i \omega_{n}=-\infty \\ \omega_{n} \neq 0}}^{\infty} \frac{1}{\left(i \omega_{n}\right)^{j}}=\lim _{\zeta \rightarrow 0}\left(-\frac{1}{(j-1) !} \partial_{\zeta}^{j-1} n(\zeta)-\frac{1}{\beta \zeta^{j}}\right)
$$

provided that limit in Eq. (5.23) exist. 


\subsection{Model Green's function}

This section will be devoted towards the construction of the model Green's function which follows the density-matrix constraint of Eq. (5.2). Before proceeding in this direction, we restate the most prominent representation of the Green's function $\boldsymbol{G}\left(i \omega_{n}\right)$ i.e. the Lehmann representation of Eq.2.58) in terms of the spectral function $\boldsymbol{A}(\epsilon)$ as,

$$
G_{\alpha \beta}\left(i \omega_{n}\right)=\int d \epsilon \frac{A_{\alpha \beta}(\epsilon)}{i \omega_{n}+\mu-\epsilon}
$$

where $\mu$ is the chemical potential and the integration is over the entire energy range. The spectral function $A_{\alpha \beta}(\epsilon)$ as defined in Eq.(2.58), satisfy two important properties,

$$
\begin{aligned}
& \int d \epsilon A_{\alpha \beta}(\epsilon)=\left\langle\chi_{\alpha} \mid \chi_{\beta}\right\rangle \\
& \int d \epsilon A_{\alpha \beta}(\epsilon) n_{F}(\epsilon)=\rho_{\alpha \beta}
\end{aligned}
$$

where $n_{F}(\epsilon)=\frac{1}{1+\exp (\beta \epsilon)}$ is the Fermi function. The Fermi-function has poles at the imaginary Matsubara frequencies $\omega_{n}=\frac{(2 n+1) \pi}{\beta}$ and has the following representation,

$$
n_{F}(\epsilon)=\frac{1}{\beta} \sum_{n} \frac{\mathrm{e}^{i \omega_{n} 0^{+}}}{i \omega_{n}-\epsilon}
$$

We now focus on the construction of the model spectral functions which will be used to construct the Green's function. The spectral function provides information about the poles and their respective weights, of the Green's function. For an occupation $f$, provided that their exist a set of $N$ poles at $\left\{A_{i}(f)\right\}_{i=1}^{N}$, and their respective weights $\left\{P_{i}(f)\right\}_{i=1}^{N}$, the spectral function has the following form,

$$
\boldsymbol{A}_{\alpha \beta}(\epsilon)=\sum_{n}\left\langle\chi_{\alpha} \mid \phi_{n}\right\rangle\left\langle\phi_{n} \mid \chi_{\beta}\right\rangle \sum_{i=1}^{\mathrm{N}} P_{i}\left(f_{n}\right) \delta\left(\epsilon-A_{i}\left(f_{n}\right)\right)
$$

where $\left|\phi_{n}\right\rangle$ are the natural orbitals and $f_{n}$ are the occupations, obtained from the eigen-representation of the one-particle reduced density matrix $\boldsymbol{\rho}$.

Using the representation in Eq. (5.27), it can be shown that for the spectral function to satisfy the properties of Eq. (5.25), the weights and the poles need to satisfy the constraints,

$$
\sum_{i=1}^{N} P_{i}(f)=1
$$




$$
\sum_{i=1}^{N} P_{i}(f) n_{F}\left(A_{i}(f)\right)=f
$$

This particular representation of spectral function in Eq. (5.27) is the most appropriate when it comes to the practical usage. It provides a systematic way to manage the complexity by allowing flexibility in the decision to limit the number of poles. To this direction one proceed towards the construction of minimal models which can reproduce the given number of poles.

\subsubsection{Single-pole approximation}

In this section we construct the most trivial spectral function. We consider that for a given occupation $f$, there exists one pole which has the form,

$$
A(f)=\frac{1}{\beta} \ln \left(\frac{1-f}{f}\right)
$$

and by default the weight of this pole is one. Using the fact that $n_{F}[A(f)]=f$, this pole satisfies the constraints of Eq. (5.28). The spectral function $\boldsymbol{A}(\epsilon)$ has the following form,

$$
\boldsymbol{A}_{\alpha \beta}(\epsilon)=\sum_{n}\left\langle\chi_{\alpha} \mid \phi_{n}\right\rangle\left\langle\phi_{n} \mid \chi_{\beta}\right\rangle \delta\left(\epsilon-\frac{1}{\beta} \ln \left(\frac{1-f_{n}}{f_{n}}\right)\right)
$$

and the model Green's function is nothing but the non-interacting Green's function $\overline{\boldsymbol{G}}_{\alpha \beta}\left(i \omega_{\nu}\right)$ in Eq. (5.1), which in the eigen-representation of $\boldsymbol{\rho}$, has the form,

$$
\overline{\boldsymbol{G}}_{\alpha \beta}\left(i \omega_{\nu}\right)=\sum_{n}\left\langle\chi_{\alpha} \mid \phi_{n}\right\rangle \frac{1}{i \omega_{\nu}-\frac{1}{\beta} \ln \left(\frac{1-f_{n}}{f_{n}}\right)}\left\langle\phi_{n} \mid \chi_{\beta}\right\rangle
$$

This particluar form of the Green's function poses a serious problem in the perturbation theory for the evaluation of Luttinger-Ward functional. To understand the breakdown, let us consider the behavior of second order diagram in the series of perturbation expansion of Luttinger-Ward functional.

Using the Green's function of Eq. (5.31), the Matsubara sum over fermionic frequencies in the evaluation of polarization tensor of Eq. 5.10 can be performed analytically and attains the following form,

$$
\chi_{a b, c d}\left(i \nu_{n}\right)=\sum_{m n}\left\langle\chi_{a} \mid \phi_{m}\right\rangle\left\langle\phi_{m} \mid \chi_{b}\right\rangle\left\langle\chi_{c} \mid \phi_{n}\right\rangle\left\langle\phi_{n} \mid \chi_{d}\right\rangle \frac{n_{F}\left[A\left(f_{n}\right)\right]-n_{F}\left[A\left(f_{m}\right)\right]}{i \nu_{n}+A\left(f_{n}\right)-A\left(f_{m}\right)}(5
$$

As evident from the approximate form of Luttinger-Ward functional described in Sec. 5.2, one needs to perform the bosonic Matsubara frequency sum which also 
includes the zero frequency. At $\nu_{n}=0$, in the limit $A\left(f_{n}\right)-A\left(f_{m}\right) \rightarrow 0$, the polarization is proportional to,

$$
\sim \beta n_{F}[A(f)]\left(n_{F}[A(f)]-1\right)
$$

In the limit of large $\beta$ i.e. at small temperatures, the pole collapse at the Fermi level i.e. $A(f) \sim 0$ and therefore the Fermi function $n_{F}[A(f)] \sim \frac{1}{2}$. Therefore the zeroth frequency contribution becomes proportional to $\beta$, which leads to breakdown of the series in low temperature regime.

The physical argument behind this breakdown of the perturbation expansion is that in the limit of $T=0$, the poles of the Green's function fall at the Fermi level and thereby leading to a system with zero band width $D$. Therefore any perturbation of Luttinger-Ward functional in $U / D$ for the model Green's function given by Eq.(5.31) is bound to fail. This particular model of the Green's function can only be used in high temperature regime, where the thermal fluctuations will be stronger than any correlation effects. This makes it un-interesting for the electronic system with strong correlation effects.

\subsubsection{Two-pole approximation}

The best way to move forward is to construct the poles in such a way, that it avoids the collapse to Fermi level. To this direction, we consider, for a given occupations two poles which does not fall at the Fermi level in the low temperature regime. This requires to search for the minimal model which can enable us to construct two poles with the required property.

The simplest prototype model which works for our purpose is the Hubbard atom. The Hubbard atom is a quantum impurity which has two energy levels $\epsilon_{a}$ and $\epsilon_{b}$ and and the two electrons on the impurity repel each other via Coulomb repulsion $\Delta$. The Hamiltonian for this impurity problem has the form,

$$
\mathcal{H}_{\text {imp. }}=\epsilon_{a} \hat{c}_{a}^{\dagger} \hat{c}_{a}+\epsilon_{b} \hat{c}_{b}^{\dagger} \hat{c}_{b}+\Delta \hat{c}_{a}^{\dagger} \hat{c}_{a} \hat{c}_{b}^{\dagger} \hat{c}_{b}
$$

The single particle Green's function which has the form,

$$
\mathcal{G}_{i j}\left(i \omega_{n}\right)=-\int_{0}^{\beta} d \tau e^{i \omega_{n} \tau} \frac{\operatorname{Tr}\left(e^{-\beta \mathcal{H}_{\mathrm{imp}}} \hat{c}_{i}(\tau) \hat{c}_{j}^{\dagger}(0)\right)}{\operatorname{Tr}\left(e^{-\beta \mathcal{H}_{\mathrm{imp}}}\right)}
$$

can be evaluated analytically. For the single impurity it can be shown that offdiagonal elements of the Green's function are zero. The diagonal element $\mathcal{G}_{a a}$ on the 
other hand has the form,

$$
\begin{aligned}
\mathcal{G}_{a a}\left(i \omega_{n}\right) & =\frac{P\left(\epsilon_{a} ; \epsilon_{b}, \Delta\right)}{i \omega_{n}-\left(\epsilon_{a}-\mu\right)}+\frac{1-P\left(\epsilon_{a} ; \epsilon_{b}, \Delta\right)}{i \omega_{n}-\left(\epsilon_{a}+\Delta-\mu\right)} \\
P\left(\epsilon_{a} ; \epsilon_{b}, \Delta\right) & =\frac{1+e^{-\beta\left(\epsilon_{a}-\mu\right)}}{1+e^{-\beta\left(\epsilon_{a}-\mu\right)}+e^{-\beta\left(\epsilon_{b}-\mu\right)}\left(1+e^{-\beta\left(\epsilon_{a}+\Delta-\mu\right)}\right)}
\end{aligned}
$$

The Green's function has two poles situated at $\epsilon_{a}$ and $\epsilon_{a}+\Delta$ with $P\left(\epsilon_{a} ; \epsilon_{b}, \Delta\right)$ and $1-P\left(\epsilon_{a} ; \epsilon_{b}, \Delta\right)$ as their respective weights.

The constraint equations of Eq. (5.28), attains a simple form,

$$
f=\frac{P\left(\epsilon_{a} ; \epsilon_{b}, \Delta\right)}{1+e^{\beta\left(\epsilon_{a}-\mu\right)}}+\frac{1-P\left(\epsilon_{a} ; \epsilon_{b}, \Delta\right)}{1+e^{\beta\left(\epsilon_{a}+\Delta-\mu\right)}}
$$

The Eq. (5.37) needs to be inverted to obtain pole $\epsilon_{a}$ as a function of the occupation $f$. However, there exist freedom on the choice of second energy level $\epsilon_{b}$.

\section{Case of $\epsilon_{a}=\epsilon_{b}$}

The most obvious choice for the second energy level $\epsilon_{b}$, is $\epsilon_{b}=\epsilon_{a}$. The constraint Eq. (5.37) can be inverted and the two poles $\left(\epsilon_{a}\right.$ and $\left.\epsilon_{a}+\Delta\right)$ and their respective weights attains the following form,

$$
\begin{aligned}
& P_{1}(f)=1-f=1-P_{2}(f) \\
& A_{1}(f)=\epsilon_{a}=\mu+\frac{1}{\beta} \log \left[\frac{1-2 f+\sqrt{\left((1-2 f)^{2}+4 f(1-f) e^{-\beta \Delta}\right)}}{2 f}\right] \\
& A_{2}(f)=A_{1}(f)+\Delta
\end{aligned}
$$

We are in a position to analyze the behavior of two poles at low temperatures or large $\beta$. For a finite value of interaction $\Delta$, and assuming $\frac{2 f_{n}\left(1-f_{n}\right) e^{-\beta \Delta}}{\left(1-2 f_{n}\right)^{2}} \sim 0$, which is valid for $f \neq 0$, we expand the square root in Eq. (5.38) to first order and obtain,

$$
A_{1}(f)=\mu+\frac{1}{\beta} \log \left[\frac{(1-2 f)+|1-2 f|\left(1+\frac{2 f(1-f) e^{-\beta \Delta}}{(1-2 f)^{2}}\right)}{2 f}\right]
$$

The two poles now has the following form,

$$
A_{1}= \begin{cases}\frac{1}{\beta} \log \left[\frac{1-2 f}{f}\right] & \text { for } f<\frac{1}{2} \\ -\frac{\Delta}{2} & \text { for } f=\frac{1}{2} \\ \frac{1}{\beta} \log \left[\frac{1-f}{|1-2 f|}\right]-\Delta & \text { for } f>\frac{1}{2}\end{cases}
$$




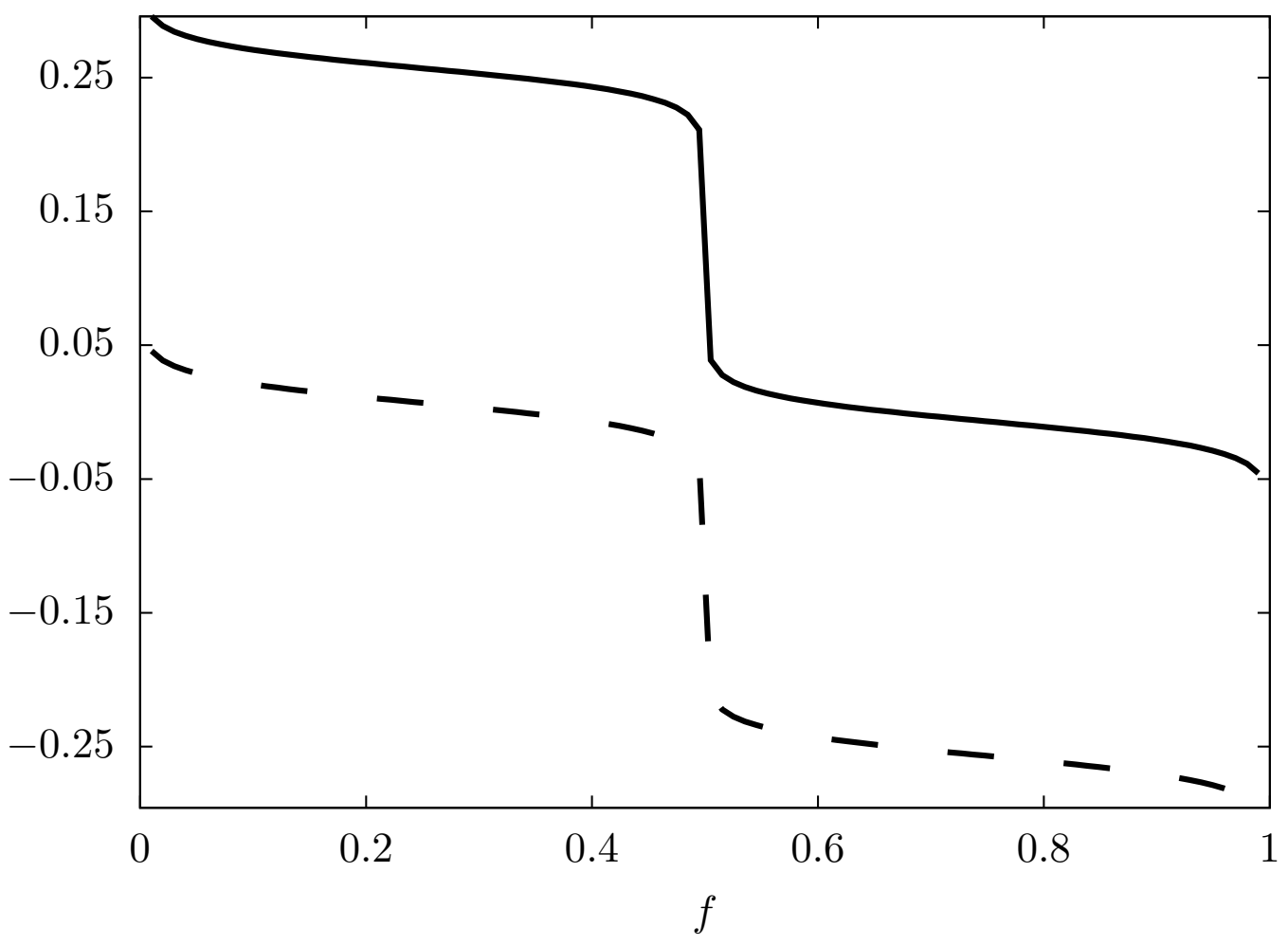

Figure 5.6: Position of poles $A_{1}(f)$ (dashed line) and $A_{2}(f)$ (solid line) as a function of occupation. The value of onsite interaction is $\Delta=0.25$ and the inverse temperature is $\beta=100$. The two poles are always separated by $\Delta$ at any given occupation.

$$
A_{2}= \begin{cases}\frac{1}{\beta} \log \left[\frac{1-2 f}{f}\right]+\Delta & \text { for } f<\frac{1}{2} \\ \frac{\Delta}{2} & \text { for } f=\frac{1}{2} \\ \frac{1}{\beta} \log \left[\frac{1-f}{|1-2 f|}\right] & \text { for } f>\frac{1}{2}\end{cases}
$$

As evident from Fig. 5.6, one of the two poles fall are at the Fermi level for all occupations except at $f=\frac{1}{2}$. The most evident choice of $\epsilon_{b}$, leads to a pole structure which does not serve our purpose.

Case of $\epsilon_{b}=\mu-\Delta / 2$

In this attempt, the energy level $\epsilon_{b}$ is placed at half the interaction strength $\Delta$ below the Fermi level i.e. $\epsilon_{b}=\mu-\frac{\Delta}{2}$. With this choice of $\epsilon_{b}$, the solution for the poles and 


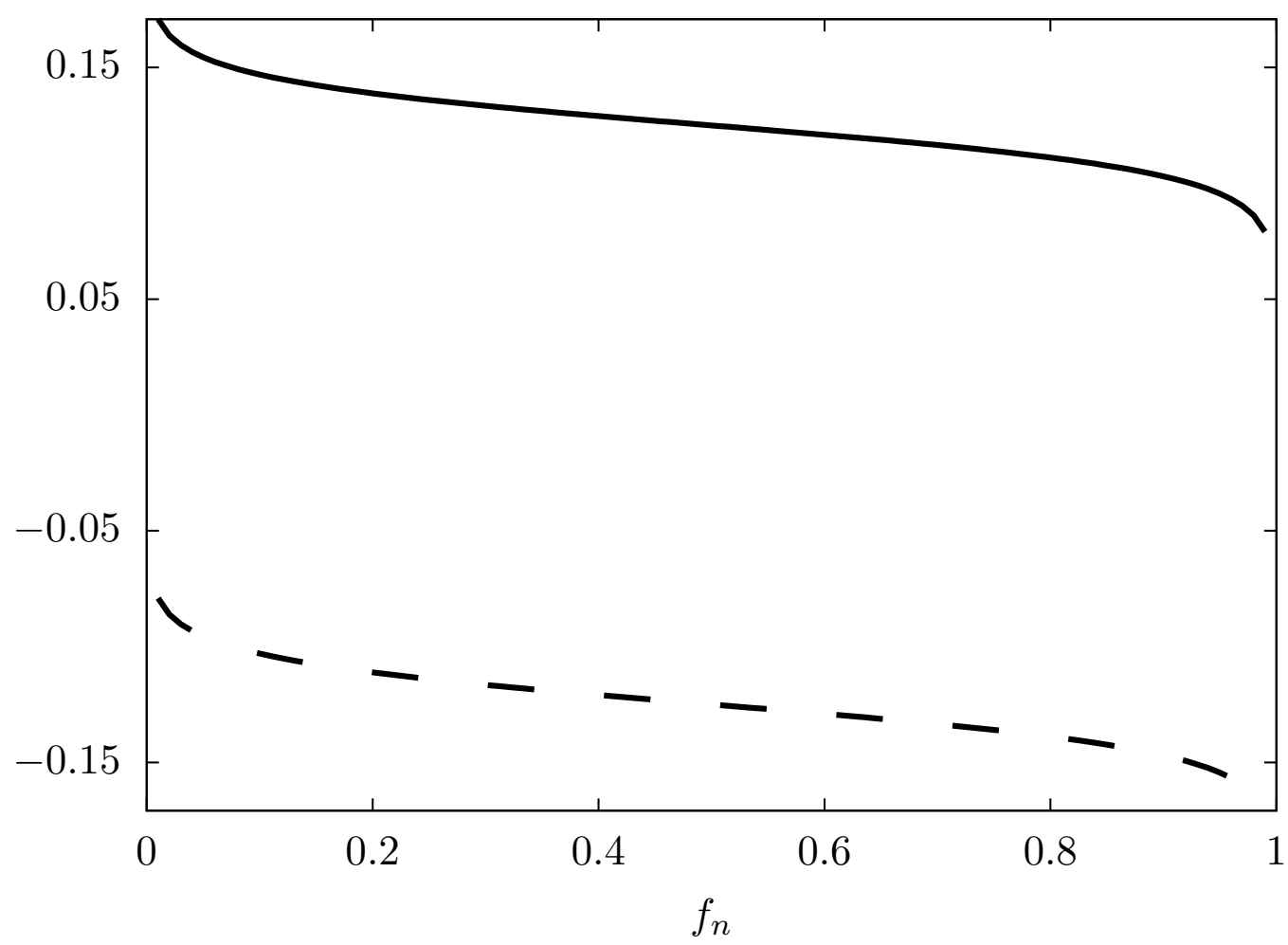

Figure 5.7: Position of poles $A_{1}(f)$ (dashed line) and $A_{2}(f)$ (solid line) as a function of occupation. The position of energy level $\epsilon_{b}$ of Eq.(5.34) has been set to $\epsilon_{b}=\mu-\frac{\Delta}{2}$. The value of onsite interaction is $\Delta=0.25$ and the inverse temperature is $\beta=100$. The two poles are always separated by $\Delta$ at any given occupation.

their respective weights in Eq. (5.37), has the form,

$$
\begin{aligned}
P(f) & =\frac{f+(1-f) \mathrm{e}^{\beta \frac{\Delta}{2}}}{1+\mathrm{e}^{\beta \frac{\Delta}{2}}} \\
A_{1}(f) & =\mu+\frac{1}{\beta} \ln \left(\frac{1-f}{f}\right)-\frac{\Delta}{2} \\
A_{2}(f) & =\mu+\frac{1}{\beta} \ln \left(\frac{1-f}{f}\right)+\frac{\Delta}{2}
\end{aligned}
$$

In Fig. 5.7, the position of two poles are plotted as a function of occupation. As evident, at low temperatures the two poles don't collapse at the Fermi level. They are always separated by interaction $\Delta$. This particular model of the Green's function helps to avoid the polarization catastrophe in the perturbative expansion of 
Luttinger-Ward functional. We will test this model Green's function in the evaluation of density matrix functional and will further test its quality by benchmarking against the results obtained from state of the art parametrized density matrix functionals.

\subsection{Performance of the density matrix functional}

This section will be devoted to the quality of approximate density matrix functional suggested in Eq. (5.6), along with the model Green's function proposed in Sec. 5.3.2. To keep the discussion in this chapter self-contained, we reiterate the definition of the grand canonical potential. For a many-particle system, the grand-canonical potential is expressed with the help of the density-matrix functional $F_{\beta}^{\hat{W}}[\boldsymbol{\rho}]$ as

$$
\begin{aligned}
\Omega_{\beta, \mu}^{\hat{h}+\hat{\boldsymbol{W}}}= & \min _{f_{n} \in[0,1],\left|\phi_{n}\right\rangle}\left\{\sum_{n} f_{n}\left\langle\phi_{n}|\hat{h}| \phi_{n}\right\rangle-\mu \sum_{n} f_{n}+F_{\beta}^{\hat{W}}\left[\sum_{n} f_{n}\left|\phi_{n}\right\rangle\left\langle\phi_{n}\right|\right]\right. \\
& \left.-\sum_{m n} \Lambda_{m n}\left(\left\langle\phi_{n} \mid \phi_{m}\right\rangle-\delta_{m n}\right)\right\}
\end{aligned}
$$

where $\hat{h}=\sum_{\alpha, \beta}\left|\chi_{\alpha}\right\rangle h_{\alpha, \beta}\left\langle\chi_{\beta}\right|$.

The reduced density-matrix functional $F_{\beta}^{\hat{W}}[\boldsymbol{\rho}]$ which will be used in the evaluation of the grand-canonical potential is given by Eq. (5.6). The chemical potential $\mu$ is a Lagrange multiplier that constrains the electron number to $N . \Lambda_{m n}$ are the Lagrange multipliers which enforce the orthogonality of the natural orbitals $\left|\phi_{m}\right\rangle$.

The density matrix functional $F_{\beta}^{\hat{W}}[\boldsymbol{\rho}]$ in Eq. (5.6), has an algebraic form for a given model Green's function. The corresponding spectral function $\boldsymbol{A}(\epsilon)$ is given in terms of poles and weights as described in Eq. (5.41). The optimization in Eq. (5.42), is done in the Car-Parinello spirit using a fictitious Lagrangian as described in Chapter. 3 and to guarantee the unbiased implementation, the method in Sec. 4.3 of Chapter. 4 is used.

As usual, the Hubbard dimer (See Sec. 2.7.1) will be used as a test system to study the quality of approximate density matrix functional.

\subsubsection{Approximate density matrix functional}

The density matrix functional in Eq. (5.6), requires the evaluation of the LuttingerWard functional. In this section we will approximate the Luttinger-Ward functional through some of the important diagrams of Sec. 5.2, namely the Hartree-Fock and higher order diagrams.

The model Green's function to be used for the evaluation of these diagrams has 
the form,

$$
G_{\alpha \beta}\left(i \omega_{\nu}\right)=\sum_{n}\left\langle\chi_{\alpha} \mid \phi_{n}\right\rangle \sum_{i=1}^{2} \frac{P_{i}\left(f_{n}\right)}{i \omega_{\nu}-A_{i}\left(f_{n}\right)}\left\langle\phi_{n} \mid \chi_{\beta}\right\rangle
$$

where the position of the poles $A_{i}$ and their respective weights $P_{i}$ are given by Eq. 5.41.

As evident from Sec. 5.2, beyond the Hartree-Fock approximation, the evaluation of the higher order approximations for the Luttinger-Ward functional requires the knowledge of the polarization tensor $\chi$. The fermionic Matsubara frequency sum in the evaluation of evaluation of polarization tensor in Eq. (5.10) can be performed analytically for the model Green's function of Eq. (5.43). The polarization tensor $\chi$ attains a simple form,

$$
\begin{aligned}
\chi_{a b, c d}\left(i \nu_{n}\right) & =\sum_{m n}\left\langle\chi_{a} \mid \phi_{m}\right\rangle\left\langle\phi_{m} \mid \chi_{b}\right\rangle\left\langle\chi_{c} \mid \phi_{n}\right\rangle\left\langle\phi_{n} \mid \chi_{d}\right\rangle \mathcal{P}\left(f_{m}, f_{n}\right) \\
\mathcal{P}\left(f_{m}, f_{n}\right) & =\sum_{i, j=1}^{N} P_{i}\left(f_{m}\right) P_{j}\left(f_{n}\right) \frac{n_{F}\left[A_{j}\left(f_{n}\right)\right]-n_{F}\left[A_{i}\left(f_{m}\right)\right]}{i \nu_{n}+A_{j}\left(f_{n}\right)-A_{i}\left(f_{m}\right)}
\end{aligned}
$$

where $\mathcal{P}\left(f_{m}, f_{n}\right)$ is the polarization coefficient which depends solely on the occupations $f_{m}$. The polarization tensor appears implicitly in the evaluation of the Luttinger-Ward functional $\Phi_{\beta}^{\text {approx }}[\boldsymbol{G}[\boldsymbol{\rho}], \hat{W}]$ (See Sec. 5.2 and therefore leads to its dependence on the occupations and the natural orbitals. Here $\Phi_{\beta}^{\text {approx }}[\boldsymbol{G}[\boldsymbol{\rho}], \hat{W}]$ is the correction to the Luttinger-Ward functional beyond the Hartree-Fock approximation.

The approximate density-matrix functional, therefore can be represented in the eigen-representation of one-particle density matrix $\boldsymbol{\rho}$, as

$$
\begin{aligned}
F_{\beta}^{\hat{W}}\left[\sum_{n} f_{n}\left|\phi_{n}\right\rangle\left\langle\phi_{n}\right|\right] & =\frac{1}{\beta} \sum_{n}\left[f_{n} \ln \left(f_{n}\right)+\left(1-f_{n}\right) \ln \left(1-f_{n}\right)\right] \\
& +\frac{1}{2} \sum_{\alpha \beta \gamma \delta}\left(W_{\alpha \beta, \delta \gamma}-W_{\alpha \beta, \gamma \delta}\right) \sum_{m n} f_{m} f_{n}\left\langle\chi_{\delta} \mid \phi_{m}\right\rangle\left\langle\phi_{m} \mid \chi_{\alpha}\right\rangle\left\langle\chi_{\gamma} \mid \phi_{n}\right\rangle\left\langle\phi_{n} \mid \chi_{\beta}\right\rangle \\
& +\Phi_{\beta}^{\text {approx }}[\boldsymbol{G}[\boldsymbol{\rho}], \hat{W}] \\
& -\frac{1}{\beta} \sum_{\omega_{n}} \sum_{m}\left\{-\ln \left[\left(i \omega_{n}-\frac{1}{\beta} \ln \left(\frac{1-f_{m}}{f_{m}}\right)\right) \sum_{i=1}^{2} \frac{P_{i}\left(f_{m}\right)}{i \omega_{n}-A_{i}\left(f_{m}\right)}\right]\right. \\
& \left.+\left(i \omega_{n}-\frac{1}{\beta} \ln \left(\frac{1-f_{m}}{f_{m}}\right)\right) \sum_{i=1}^{2} \frac{P_{i}\left(f_{m}\right)}{i \omega_{n}-A_{i}\left(f_{m}\right)}-1\right\}
\end{aligned}
$$

The sum over fermionic and bosonic Matsubara frequencies in Eq. (5.45) is performed numerically, employing the regularization technique as discussed in Sec . 5.2.4 


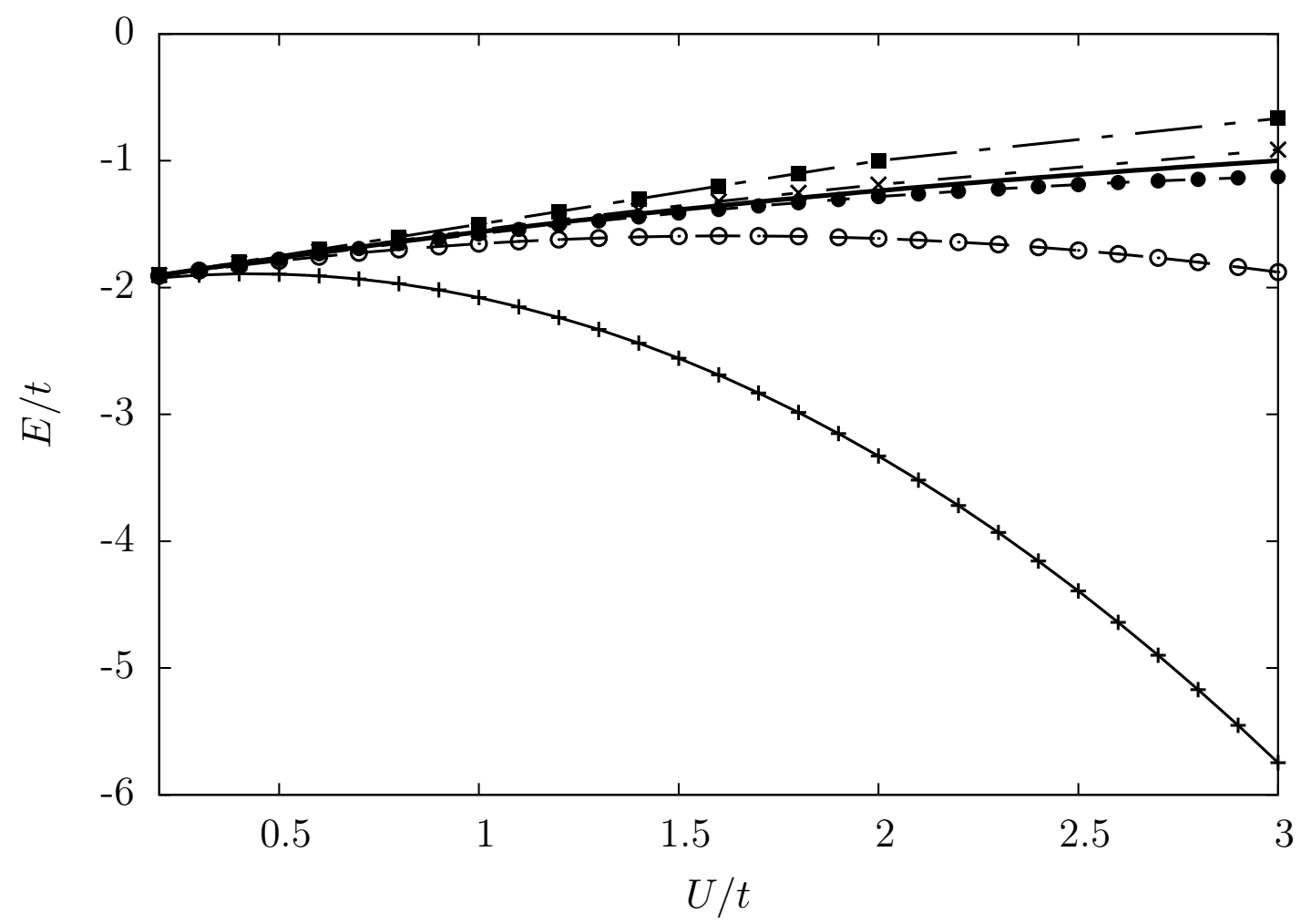

Figure 5.8: The ground-state energy for the half-filled Hubbard dimer as a function of interaction strength $U / t$ for different approximations of density-matrix functionals. Solid line: exact ground state energy. Solid square: Hartree-Fock density matrix functional. Crosses: power-functional with $\alpha=0.53$. Solid circles: approximate density matrix functional of Eq. 5.45 with $\Delta / t=1.8$. Open circles: Approximate density matrix functional of Eq. (5.45) with $\Delta / t=0.8$. Solid triangles: approximate density matrix functional of Eq. (5.45) with $\Delta / t=0.2$. The value of inverse temperature is $\beta=1000$

\subsubsection{Results}

With the given form of the density-matrix functional in Eq. (5.45), the grandcanonical potential is evaluated from Eq. (5.42), for the Hubbard dimer at halffilling. One important point to note is that the approximate density-matrix functional depends parametrically on pole separation $\Delta$ through its dependence on the poles position $A_{i}$. In this section, the results for the grand-canonical potential will be examined for different values of $\Delta$. The consistent procedure to fix the value of $\Delta$ will be discussed later. 


\section{Case of $\Phi_{\beta}^{\text {approx }}[\boldsymbol{G}[\boldsymbol{\rho}], \hat{W}]=\Phi_{\beta}^{(2)}[\boldsymbol{G}[\boldsymbol{\rho}], \hat{W}]$}

In this section we consider only the second-order diagrams for the approximate Luttinger-Ward functional i.e. $\Phi_{\beta}^{\text {approx }}[\boldsymbol{G}[\boldsymbol{\rho}], \hat{W}]=\Phi_{\beta}^{(2)}[\boldsymbol{G}[\boldsymbol{\rho}], \hat{W}]$ and use it to evaluate the approximate density matrix functional of Eq. 5.45 .

In Fig. 5.8, the grand-canonical potential for the Hubbard dimer is plotted as a function of the interaction strength $U$. Hartree-Fock and the power functional ( $\alpha=0.53)$ overestimates the grand potential. The energies however obtained from the approximate density-matrix functional of Eq. (5.45) with $\Phi_{\beta}^{\text {approx }}[\boldsymbol{G}[\boldsymbol{\rho}], \hat{W}]=$ $\Phi_{\beta}^{(2)}[\boldsymbol{G}[\boldsymbol{\rho}], \hat{W}]$, are underestimated. It is also noticeable from Fig. 5.8 , that by increasing the pole-separation $\Delta$, the approximate density-matrix functional leads to better estimation of the grand potential.

Also perceptible from Fig. 5.8, is the fact that for small pole-separations, the deviation from the exact solution, starts at very small values of interaction strength $U$. The deviation can be pushed towards larger values of interaction strength by increasing the pole-separation. This behavior can indeed be understood from the fact that the small pole-separation leads to a Green's function which bears resemblance to the single-pole approximant, and therefore leads to polarization catastrophe as discussed in Sec. 5.3.1. The breakdown of the second-order perturbation theory can however be postponed to larger values of the interaction strength by increasing the pole-separation.

\section{Derivative discontinuity at half-filling}

As discussed in Sec. 4.6, the quality of an approximate density-matrix functional depends on how well it can reproduce the derivative discontinuity. The derivative discontinuity provides an estimation of the fundamental band gap. In Fig. 5.9. the chemical potential $\mu(N)=\frac{1}{t} \frac{\partial E(N)}{\partial N}$ at half-filling is plotted as a function of the particle number. The exact solution has a jump at $N=2$ (half-filling).

The Müller and the power functional $(\alpha=0.53)$ fails to reproduce the derivative discontinuity. The Hartree-Fock on the other hand under-estimate the band-gap for $U=2.5 t$, while it gives a very good estimation of band-gap for $U=3 t$. In Fig. 4.12 , calculations for $\mathrm{U}=5 t$ shows that Hartree-Fock functional over-estimates the band-gap.

Our proposed approximate density-matrix functional of Eq. (5.45) with the LuttingerWard functional approximated by the second order diagram i.e. $\Phi_{\beta}^{\text {approx }}[\boldsymbol{G}[\boldsymbol{\rho}], \hat{W}]=$ $\Phi_{\beta}^{(2)}[\boldsymbol{G}[\boldsymbol{\rho}], \hat{W}]$, fails to reproduce the derivative discontinuity. It nevertheless produces a smoothened derivative discontinuity (the chemical potential undergoes a continuous transition between two linear functions). Thereby, using the extrapolation method proposed by Sharma etal. [53], one can estimate the band-gap.

Using the extrapolation method, Müller functional still leads to a vanishing bandgap. As discussed in Chapter 4 , the power functional $(\alpha=0.53)$ leads to a finite 

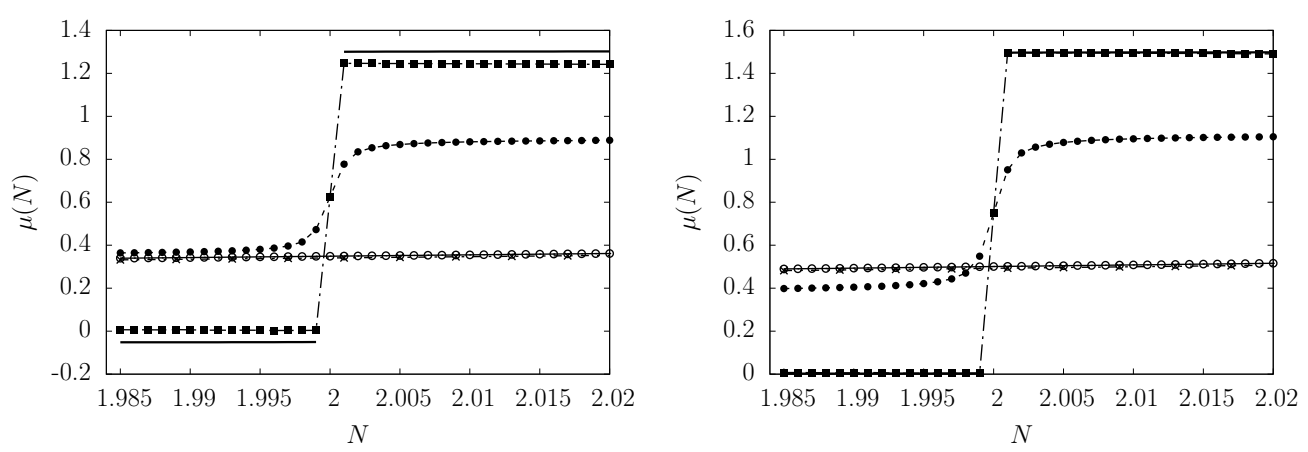

Figure 5.9: Chemical potential $\mu(N)$ of the Hubbard dimer with $U=2.5 t$ (left panel) and $U=3 t$ (right panel) in units of the hopping parameter $t$ as function of particle number $N$. Solid line: exact solution, solid squares: Hartree-Fock approximation, open circles: power functional with $\alpha=0.53$, crosses: Müller functional, solid circles: approximate density-matrix functional of Eq. (5.45). The value of inverse temperature is $\beta=1000$ and the value of pole separation is $\Delta=1.8$.

band-gap only in the anti-ferromagnetic regime. The chosen value of the interaction strength $(U=2.5 t$ and $U=3 t)$ in Fig. 5.9 is lower than the critical value of the interaction $U_{c} \sim 6 t$, beyond which power functional $(\alpha=0.53)$ produces antiferromagnetic ground-state. This explains the absence of any smoothened derivative discontinuity in case of the power functional in Fig. 5.9.

On the other hand, using the extrapolation method, the approximate densitymatrix functional of Eq. (5.45) will lead to a band-gap smaller than the exact value.

$$
\text { Case of } \Phi_{\beta}^{\text {approx }}[\boldsymbol{G}[\boldsymbol{\rho}], \hat{W}]=\Phi_{\beta}^{(2)}[\boldsymbol{G}[\boldsymbol{\rho}], \hat{W}]+\Phi_{\beta}^{\mathrm{RPA}-\mathrm{PH}}[\boldsymbol{G}[\boldsymbol{\rho}], \hat{W}]+\Phi_{\beta}^{\mathrm{RPA}-\mathrm{PP}}[\boldsymbol{G}[\boldsymbol{\rho}], \hat{W}]
$$

The next logical step to move ahead with the approximate density matrix functional in Eq. (5.45), is to improve on the approximation for the Luttinger-Ward functional $\Phi_{\beta}^{\text {approx }}[\hat{G}[\boldsymbol{\rho}], \hat{W}]$ i.e. to move beyond the second order in perturbation theory. As stated in Sec. 5.2, the most prominent way to include higher order diagrams is to select a class of diagrams which can be summed to an infinite order. One such class of diagrams forms the random-phase-approximation (RPA) series with the particlehole and particle-particle channel as discussed in Sec. 5.2.3.

In Fig. 5.10, the energies obtained from the different approximations for the Luttinger-Ward functional are plotted as a function of the interaction strength $U$. It is evident from Fig. 5.10, that the inclusion of higher order series like RPA-PH and RPA-PP does not improve the estimation of energy for the half-filled Hubbard dimer. RPA-PH and RPA-PP series breaks down at even smaller values of interaction strength compared to the second-order approximation.

It seems from the findings that the second-order approximation to the Luttinger- 
Ward functional performs better than the partial summations of certain classes of diagrams like RPA-PH and RPA-PP.

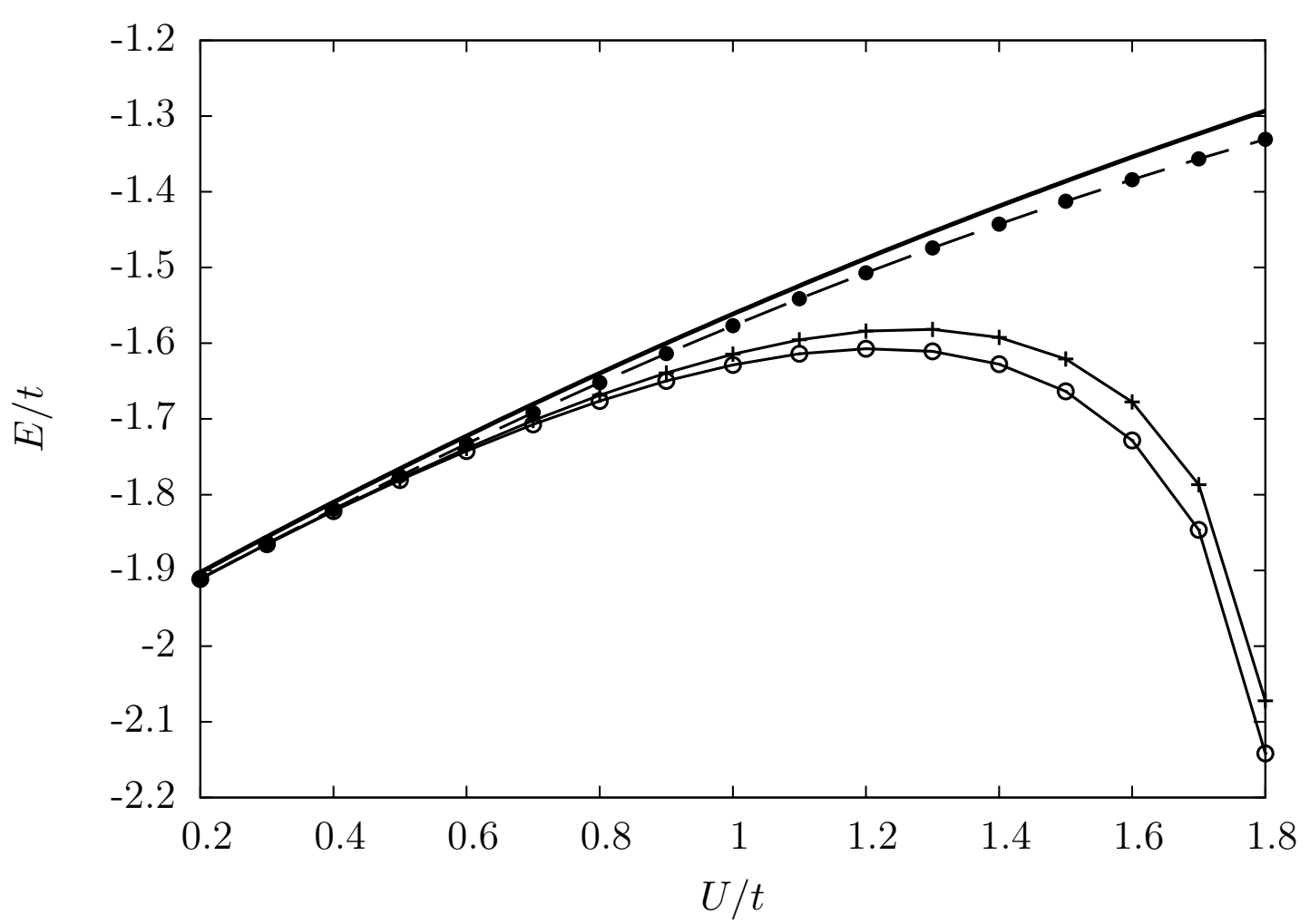

Figure 5.10: The ground-state energy for the half-filled Hubbard dimer as a function of interaction strength $U / t$ for different approximations of density-matrix functionals. Solid line: exact ground state energy. Approximate density matrix functional of Eq. 5.45 with- Solid circles: $\Phi_{\beta}^{\text {approx }}[\boldsymbol{G}[\boldsymbol{\rho}], \hat{W}]=$ $\Phi_{\beta}^{(2)}\left[\boldsymbol{G}[\boldsymbol{\rho}]\right.$. Crosses: $\Phi_{\beta}^{\mathrm{approx}}[\boldsymbol{G}[\boldsymbol{\rho}], \hat{W}]=\Phi_{\beta}^{(2)}\left[\boldsymbol{G}\left[\boldsymbol{\rho}+\Phi_{\beta}^{\mathrm{RPA}-\mathrm{PH}}[\boldsymbol{G}[\boldsymbol{\rho}]\right.\right.$. Open circles: $\Phi_{\beta}^{\text {approx }}[\boldsymbol{G}[\boldsymbol{\rho}], \hat{W}]=\Phi_{\beta}^{(2)}\left[\boldsymbol{G}\left[\boldsymbol{\rho}+\Phi_{\beta}^{\mathrm{RPA}-\mathrm{PH}}\left[\boldsymbol{G}[\boldsymbol{\rho}]+\Phi_{\beta}^{\mathrm{RPA}-\mathrm{PP}}[\boldsymbol{G}[\boldsymbol{\rho}]\right.\right.\right.$. The value of inverse temperature is $\beta=1000$ and the poles separation is $\Delta=1.8$.

\subsubsection{Consistent way to fix $\Delta$}

In the previous section, the calculations for the grand-canonical potential were performed for a fixed value of the pole-separation $\Delta$. It was found that by increasing the value of $\Delta$, the breakdown of the perturbation theory can be postponed towards larger values of the interaction strength. The parameter $\Delta$, was in a way chosen arbitrarily. 

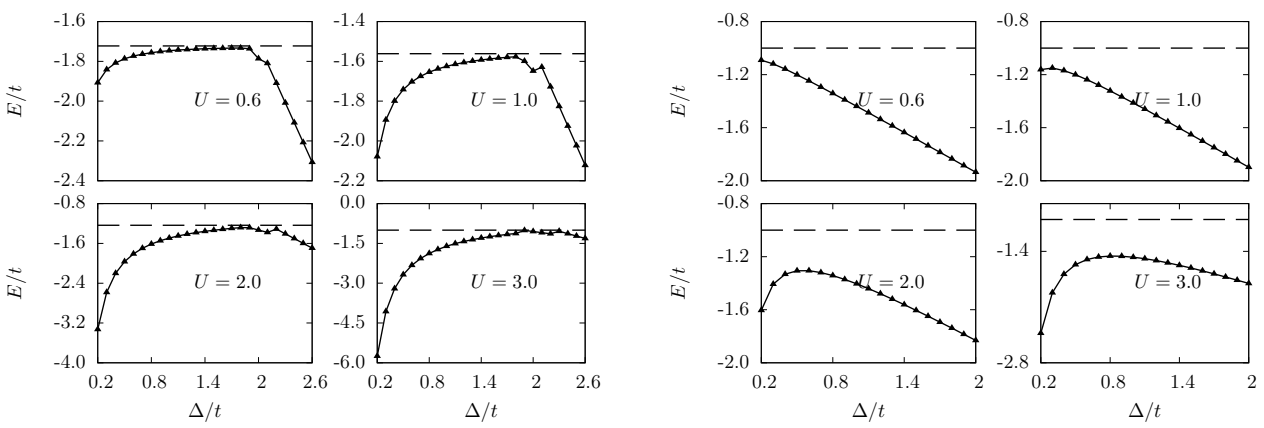

Figure 5.11: The ground-state energy for the half-filled (left panel) and quarter-filled (right panel) Hubbard dimer as a function of pole-separation $\Delta / t$ for different values of interaction strength $U / t$. Dashed line: exact ground state energy. solid lines with symbols: energy from the approximate density matrix functional of Eq. (5.45). The value of inverse temperature is $\beta=1000$.

In this section, we propose a consistent way to fix the value of $\Delta$ for the calculations. As evident from Fig. 5.11, the pole-separation $\Delta$ cannot be increased arbitrarily to larger values, as the deviation from exact value starts to increase.

For the Hubbard dimer at quarter and half-filling, the energy obtained from the approximate density-matrix functional of Eq. (5.45) as a function of pole-separation $\Delta$ is concave. At the maxima, the deviation from the exact ground-state energy is the least, as shown in Fig. 5.11.

This behavior of grand-canonical potential as a function of pole-separation $\Delta$ provides a lead towards a consistent way to fix the value of $\Delta$. Beside the minimization in Eq. (5.42), one also needs to perform a stationary point search in the space of pole-separation $\Delta$.

Also evident from Fig. 5.11, that the second-order approximation to LuttingerWard functional provides a good estimate of energy at half-filling in the given range of interaction strength $U$. At quarter-filling, the approximation provides a good estimate only for the smaller values of the interaction strength.

\subsection{Summary}

In this chapter, we presented the first results for an approximate density matrix functional constructed in a controlled way from an exact relation with the LuttingerWard functional [48]. The framework for this approximate scheme involves approximation at two different levels. The first set of approximation comes in through the construction or search for a reasonable model Green's function which helps in capturing the essential physics. The second set of approximation lies in the evalua- 
tion of the Luttinger-Ward functional for the given model Green's function, which is done through a perturbative approach.

The search for minimalistic model Green's function is done through physical reasoning. Once the Green's function is chosen, it leads to an extreme simplification where one can avoid a computationally demanding stationary point search in the space of Green's function and self-energy.

We first discussed the most simple single-pole approximation for the model Green's function and demonstrated that it leads to a polarization catastrophe. The reason for such catastrophe was the peculiar property of the pole of the Green's function which in the low temperature regime collapses to the Fermi level. This collapse of the pole to the Fermi energy leads to vanishing band-width and therefore any finite interaction strength $U$ leads to the breakdown of perturbation theory.

Learning from the failure of the single-pole approximation for the model Green's function, it became evident that one needs atleast two poles which repel each other and are situated away from the Fermi level at low temperatures for any given value of occupations. We constructed one such model Green's function which obeys the density matrix constraint and its two poles avoid any such collapse to the Fermi level. This simplest two-pole model Green's function fixes the problem of the polarization catastrophe.

With the minimal two-pole model for the Green's function, we evaluated the approximate density matrix functional using different approximations for the LuttingerWard functional. The approximate density matrix functional was used to study the Hubbard dimer. It was demonstrated that the second order approximation to the Luttinger-Ward functional provides a good estimate of the ground-state energy at half-filling. It also leads to a smoothened derivative discontinuity at half-filling. The extrapolation method provides a quantitative estimate of the fundamental band-gap and it was observed that our approximate density matrix functional underestimates the band-gap. In terms of the energy and the band-gap, our approximate functional performs better than the power and Müller functional, which leads to a vanishing band-gap. It however suffers from the breakdown of the perturbation theory beyond a certain limit of the interaction strength. Higher order approximation to the Luttinger-Ward functional are also discussed and were not found to perform better than the second-order approximation. 



\section{Conclusion and Outlook}

The broader perspective of this thesis is to present reduced density matrix functional theory (rDMFT) as a useful framework for the rigorous formulation of hybrid theories like LDA $+\mathrm{U}$ [36], DFT-plus-Gutzwiller approximation[40], DFT+DMFT [12].

It has already been established that rDMFT can make contact with many-particle wavefunction and the Green's function based approaches [7, 48]. The exact relation between rDMFT to the many-particle wave functions was established by Levy's constrained search algorithm [7] and recently efforts have been made to construct controlled approximation [47] by using the exact link. In 2013, Blöchl et al. [48, provided an exact link between rDMFT and the many-body perturbation theory through Green's function and Luttinger-Ward functional [49].

However for decades, the development in the field of rDMFT did not proceed in the direction of providing controlled approximations based on exact relations mentioned above. Rather, it concentrated on searching for model density matrix functionals which can capture the essential physical effects while having an algebraic dependence of the energy on the one-particle reduced density matrix.

The significant aspect that came to the forefront from the work presented in this thesis was the necessity to put effort in building controlled approximations on theories which make exact contact with the many-particle description of the electronic system.

The two key issues that we address in this thesis are the following,

1. Performance of a class of state-of-the-art reduced density matrix functionals namely the Hartree-Fock, Müller [50, 51] and the power functional [53].

2. Efficient framework for an approximate scheme, built on the exact relation between density-matrix functional and the Luttinger-Ward functional [48]

The basic machinery required at the very onset of addressing the above mentioned issues are the model systems and the optimization schemes.

We use simple model systems like the Hubbard dimer, Hubbard chains and rings as the benchmark systems. For most cases we used Hubbard dimer as a test system because the large interaction limit $U \rightarrow \infty$, describes the bond dissociation limit of the hydrogen molecule and the most prominent failure of the density functionals occurs during the bond dissociation. This simple system captures the essential features 
of the correlation effects present in a chemical bond. We emphasize particularly on the left-right correlation, which is a dominant correlation effect in the dissociation problem and which is not captured easily by density functionals [28].

An unbiased optimization scheme in the spirit of Car-Parinello was employed to optimize the natural orbitals and the occupations and more importantly it allows for non-collinear spin polarized states. This strategy grants flexibility in bringing out the potential flaws of the density matrix functionals when studying the electronic properties of real materials.

Equipped with the basic machinery, we first benchmark a class of state of the art density matrix functionals namely the Hartree-Fock, Müller [50, 51] and the power functional [53].

With the knowledge of the exact solution for a half-filled Hubbard dimer, we started studying the Hartree-Fock functional for the Hubbard model[44, 45, 46]. The Hartree-Fock functional is analogous to hybrid density functionals [23], which admix a portion of exact exchange to the exchange-correlation energy. Our results [56] showed that the Hartree-Fock functional leads to an anti-ferromagnetic state with non-vanishing local moments. The transition to an anti-ferromagnetic state is abrupt in the Hartree-Fock case, unlike a smooth and continuous build up to anti-ferromagnetic correlations in the exact scenario.

The Müller functional [50] on the other hand leads to a one-dimensional manifold [56] of ferromagnetic solutions which are degenerate with the exact non-magnetic solution. This degeneracy leads to an infinite magnetic susceptibility in contrast to vanishing zero-temperature susceptibility of the exact solution.

This suggests that extreme care need to be taken when performing large scale electronic structure calculations with Müller functional, as it has the potential to produce incorrect magnetic properties.

The power functional [53], which interpolates between Müller and the HartreeFock functional produces Müller like solutions at small interaction strength. Of the one-dimensional manifold, power functional leads to states with maximal ferromagnetic moment. At large interaction, the solution is a non-collinear anti-ferromagnet. The transition to an anti-ferromagnetic state occurs at critical interaction strength $U_{c}(\alpha)$, which falls off rapidly from $U_{c}\left(\alpha=\frac{1}{2}\right)=\infty$ in case of Müller to $U_{c}(\alpha=1)=2 t$ for the Hartree-Fock. These problems of artificial magnetic transition and infinite magnetic susceptibility were also shown [56] to persist in extended systems like Hubbard rings and systems with non-local Coulomb interactions.

Besides the problem of bond-dissociation, we [56] also investigated the issue of derivative discontinuity [94, 28. The derivative discontinuity also gives an estimate of the fundamental band-gap. The Müller functional [50] leads to a metal like behavior as it describes the Hubbard dimer with a vanishing band gap. The power functional [53] also lacks derivative discontinuity. However in non-collinear calculations, in the anti-ferromagnetic regime, the chemical potential obtained with the 
power functional undergoes a continuous transition between two linear functions of the particle number. The offset between the linear functions can be exploited to extract the band-gap [53, 91, 95, 89, 90]. We demonstrated [56] that the band gap obtained from this method can be tuned from zero to the Hartree-Fock result, by changing the parameter $\alpha$ of the power functional. In non-magnetic calculations, the band-gap opens up at larger values of the parameter $\alpha$, than in the magnetic calculations.

The tunability of the band gap is similar to other methods such as LDA+U [36] and hybrid density functionals [23]. However, the latter methods exhibit a true derivative discontinuity and their band gap does not shrink below the Kohn-Sham band gap, which is analogous to the non-interacting band gap of the Hubbard dimer.

Our findings [56] demonstrate the necessity to look for approaches which make direct contact with the many-particle description of the electronic system. To this direction, we presented in Chapter 5, a promising approximate scheme, built on the exact relation between density-matrix functional and the Luttinger-Ward functional 48 .

In Chapter 5, we present a two level approximate scheme which starts with constructing an approximate model Green's function that obeys the density matrix constraint. This eliminates the computationally demanding stationary point search in the space of Green's function and the self-energy. The second approximation is in the perturbative expansion of the Luttinger-Ward functional [49].

While searching for an approximate model Green's function, we arrived at a minimal two-pole model Green's function. The two-pole model Green's function eliminates the polarization catastrophe problem present for the single-pole approximation of the Green's function.

With the second-order approximation of the Luttinger-Ward functional evaluated for the two-pole model Green's function, our approximate density matrix functional provides a good estimate of the ground state energy of the Hubbard dimer at halffilling for small to intermediate interactions $U \sim 3 t$. For interaction strength larger than $3 t$ leads to the breakdown of second-order perturbation theory. Our approximate density matrix functional also produces a smoothened derivative discontinuity in the permissible range of interactions where the Müller and the power functional leads to a vanishing band-gap. Higher order approximations to the Luttinger-Ward functional like the random-phase-approximation (RPA) surprisingly do not improve the ground state energy.

The development of approximations through the Green's function based method is not yet complete. It is an ongoing project that needs more careful study to analyze and improve on the current form of the approximate density matrix functional provided in Chapter 5. From the current status of the approximate density-matrix functional, there are still certain open questions that need to be addressed. The most pertinent one is the comparison of the performance of random-phase-approximation 
with the second-order approximation to the Luttinger-Ward functional. This might also require the comparison of the exact Green's function for the Hubbard dimer with the two-pole model Green's function evaluated from the ground-state one-particle density matrix obtained from the approximate density-matrix functional presented in Chapter 5.

To improve on the approximation of the density-matrix functional, we need a proper understanding of the higher order approximations of the Luttinger-Ward functional obtained from partial summations of a specific class of diagrams (RPA etc). Once it has been accomplished, we can consider better and more sophisticated models for the Green's function.

We believe that the approximate density matrix functional presented in Chapter 5, can be a good starting point to study materials with weak to intermediate correlations. The development in this direction is still under progress and there is lot of room for the improvement. 
Appendices 



\section{A Evaluation of derivative matrix $D$}

The derivative matrix $\boldsymbol{D}=\frac{\partial E}{\partial \boldsymbol{\rho}}$ is one of the important entity in the Car-Parinello simulations (See. Chapter. 3). The energy functional is generally evaluated in the eigen representation of the one-particle density matrix $\boldsymbol{\rho}$ and therefore the derivatives are available with respect to occupations and the natural orbitals.

To obtain the derivatives with respect to $\boldsymbol{\rho}$, we evaluate the first order variation in occupation and natural orbital with a first order change in the density matrix. To this effect we write the eigen decomposition of $\boldsymbol{\rho}$ as,

$$
f=U^{\dagger} \rho \boldsymbol{U}
$$

where $\boldsymbol{f}$ is a diagonal matrix whose elements are the occupations $f_{n}$ and $\boldsymbol{U}$ is a unitary matrix which contains the natural orbitals $\left|\phi_{n}\right\rangle$ as its columns.

The first order variation of Eq. A.1 has the form

$$
d \boldsymbol{f}=d \boldsymbol{U}^{\dagger} \boldsymbol{\rho} \boldsymbol{U}+\boldsymbol{U}^{\dagger} d \boldsymbol{\rho} \boldsymbol{U}+\boldsymbol{U}^{\dagger} \boldsymbol{\rho} d \boldsymbol{U}
$$

Using the fact that the matrix $\boldsymbol{U}$ is a unitary matrix and hence $d\left(\boldsymbol{U}^{\dagger} \boldsymbol{U}\right)=0$, we can rewrite Eq. A.2 as

$$
d \boldsymbol{f}+\left(\boldsymbol{U}^{\dagger} d \boldsymbol{U}\right) \boldsymbol{f}-\boldsymbol{f} \boldsymbol{U}^{\dagger} d \boldsymbol{U}=\boldsymbol{U}^{\dagger} d \boldsymbol{\rho} \boldsymbol{U}
$$

Comparing the diagonal and non-diagonal elements on both sides of Eq. A.3 we obtain,

$$
\begin{aligned}
d f_{i} & =\left(\boldsymbol{U}^{\dagger} d \boldsymbol{\rho} \boldsymbol{U}\right)_{i i} \\
\left(\boldsymbol{U}^{\dagger} d \boldsymbol{U}\right)_{i j} & =\frac{\left(\boldsymbol{U}^{\dagger} d \boldsymbol{\rho} \boldsymbol{U}\right)_{i j}}{f_{j}-f_{i}}
\end{aligned}
$$

and therefore the first order variation of the occupation and natural orbitals has the form,

$$
\begin{aligned}
d f_{i} & =\left(\boldsymbol{U}^{\dagger} d \boldsymbol{\rho} \boldsymbol{U}\right)_{i i} \\
d \boldsymbol{U}_{i j} & =\sum_{k \neq j} U_{i k} \frac{\left(\boldsymbol{U}^{\dagger} d \boldsymbol{\rho} \boldsymbol{U}\right)_{k j}}{f_{j}-f_{k}}
\end{aligned}
$$


The first order deviation of the natural orbital assume that the eigenvalues of density matrix i.e. the occupations are non-degenerate. However it can be shown without loss of generality that even in the case of degenrate occupations, the first order correction to natural orbitals is given by Eq. A.5 with sum over non-degenerate states only.

The first order variation of energy functional has the form,

$$
\begin{aligned}
d E & =\sum_{i} \frac{\partial E}{\partial f_{i}} d f_{i}+\sum_{i j} \frac{\partial E}{\partial U_{i j}} d U_{i j}+\sum_{i j} \frac{\partial E}{\partial U_{i j}^{*}} d U_{i j}^{*} \\
& =\sum_{i} \frac{\partial E}{\partial f_{i}}\left(\boldsymbol{U}^{\dagger} d \boldsymbol{\rho} \boldsymbol{U}\right)_{i i}+\sum_{i j} \frac{\partial E}{\partial U_{i j}} \sum_{k \neq j} U_{i k} \frac{\left(\boldsymbol{U}^{\dagger} d \boldsymbol{\rho} \boldsymbol{U}\right)_{k j}}{f_{j}-f_{k}} \\
& +\sum_{i j} \frac{\partial E}{\partial U_{i j}^{*}}\left(\sum_{k \neq j} U_{i k} \frac{\left(\boldsymbol{U}^{\dagger} d \boldsymbol{\rho} \boldsymbol{U}\right)_{k j}}{f_{j}-f_{k}}\right)^{*} \\
& =\sum_{m n}\left[\sum_{k \neq j} U_{m k} \frac{1}{f_{j}-f_{k}} \sum_{i}\left(\frac{\partial E}{\partial U_{i j}} U_{i k}-\frac{\partial E}{\partial U_{i k}^{*}} U_{i j}^{*}\right) U_{n j}+\sum_{i} \frac{\partial E}{\partial f_{i}} U_{m i}^{*} U_{n i}\right] d \rho_{m n}
\end{aligned}
$$

Thus once the derivatives of energy functional with respect to occupations and natural orbitals are available we can evaluate the elements of derivative matrix $D_{n m}=\frac{\partial E}{\partial \rho_{m n}}$ using the transformation given in Eq. A.6 as,

$$
D_{n m}=\sum_{k \neq j} U_{m k} \frac{1}{f_{j}-f_{k}} \sum_{i}\left(\frac{\partial E}{\partial U_{i j}} U_{i k}-\frac{\partial E}{\partial U_{i k}^{*}} U_{i j}^{*}\right) U_{n j}+\sum_{i} \frac{\partial E}{\partial f_{i}} U_{m i}^{*} U_{n i}
$$

The derivative matrix $\boldsymbol{D}$ is a central entity to the Car-Parinello simulations and it needs to be evaluated efficiently. 


\section{B Unconstrained Optimization}

The evaluation of grand-canonical potential as,

$$
\begin{aligned}
\Omega_{\beta}^{\hat{\boldsymbol{h}}+\hat{\boldsymbol{W}}}= & \min _{f_{n} \in[0,1],\left|\phi_{n}\right\rangle}\left\{\sum_{n} f_{n}\left\langle\phi_{n}|\hat{h}| \phi_{n}\right\rangle-\mu \sum_{n} f_{n}+F_{\beta}^{\hat{W}}\left[\sum_{n} f_{n}\left|\phi_{n}\right\rangle\left\langle\phi_{n}\right|\right]\right. \\
& \left.-\sum_{m n} \Lambda_{m n}\left(\left\langle\phi_{n} \mid \phi_{m}\right\rangle-\delta_{m n}\right)\right\}
\end{aligned}
$$

is a constrained optimization problem. As shown in Chapter. 3, to fulfill the constraint of orthogonalization of natural orbitals in each time step, one needs to solve iteratively, a non-linear set of matrix equations. An efficient rDMFT driver, based on Car-Parinello optimization has been implemented, which deals with the natural orbitals constraints efficiently.

We also use efficient multi-dimensional minimization solvers available in numerical libraries like GNU Scientific Library (GSL) [103] and NLopt [104] . These libraries consists of solvers based on efficient minimization algorithms like steepest descent algorithm, quasi-Newton method like BFGS algorithm (Broyden- Fletcher- GoldfarbShanno), Fletcher-Reeves conjugate gradient algorithms, Simplex algorithm etc.

These solvers are used to carry out unconstrained optimization in a multi-dimensional space. To make an efficient use these solvers, we need to to reframe our problem of finding the minima of energy functional as described in Eq. B.1. To convert the constrained optimization problem of Eq. B.1 to an unconstrained optimization problem we have to get rid of the orthonormality constraint of the natural orbitals.

Using the fact that the matrix $\boldsymbol{U}$ is a unitary matrix i.e. $\boldsymbol{U}^{\dagger} \boldsymbol{U}=\mathbf{1}$, it can can parametrized by a set of real parameters $([105,106])$.

The parametrization relies on the existence of Euler angle parametrization of representations of $\mathrm{SU}(\mathrm{N})$ algebra. The linear independent set of $N^{2}-1$ traceless hermitean matrices $\boldsymbol{\lambda}_{i}$ forms a representation of $\mathrm{SU}(\mathrm{N})$ Lie algebra, and has the form [106],

$$
\begin{aligned}
{\left[\boldsymbol{\lambda}_{i}, \boldsymbol{\lambda}_{j}\right] } & =2 i f_{i j k} \boldsymbol{\lambda}_{k} \\
f_{i j k} & =\frac{1}{4 i} \operatorname{Tr}\left(\left[\left[\boldsymbol{\lambda}_{i}, \boldsymbol{\lambda}_{j}\right] \boldsymbol{\lambda}_{k}\right]\right)
\end{aligned}
$$

where [,] is the commutator and $f_{i j k}$ are the structure constants. 
It was shown by Tilma et.al [106], that any unitary matrix of dimension $\mathrm{N}$ can be parametrized using $N^{2}-1$ real parameters $\left\{\alpha_{i}\right\}$ as,

$$
\begin{aligned}
& \boldsymbol{U}=\prod_{N \geq m \geq 2}\left(\prod_{2 \leq k \leq m} \boldsymbol{A}(k, j(m))\right) \\
& \times e^{\boldsymbol{\lambda}_{3} \alpha_{N^{2}-(N-1)}} \ldots e^{\boldsymbol{\lambda}_{(N-1)^{2}-1} \alpha_{N^{2}-2}} e^{\boldsymbol{\lambda}_{N^{2}-1} \alpha_{N^{2}-1}} \\
& \boldsymbol{A}(k, j(m))=e^{\boldsymbol{\lambda}_{3} \alpha_{2 k-3+j(m)}} e^{\boldsymbol{\lambda}_{(k-1)}{ }^{2}+1} \alpha_{2 k-2+j(m)} \\
& j(m)= \begin{cases}0, & \text { for } m=N \\
\sum_{0 \leq l \leq N-m-1} 2(m+l), & \text { for } m \neq N\end{cases}
\end{aligned}
$$

By construction it is evident that $\boldsymbol{U}$ is hermitean. It is also evident from Eq. (B.3) that not all $\boldsymbol{\lambda}$ matrices appear in the expression. The recipe for constructing important $\boldsymbol{\lambda}$ matrices used in the representation of unitary matrix $U$ is given by Tilma etal. [106].

For $N=2$ the matrices are the simple Pauli matrices which are the representation for generators of $\mathrm{SU}(2)$ algebra. For $N>2$, the $\boldsymbol{\lambda}$ matrices are generated by the procedure as follows,

$$
\begin{aligned}
& \lambda_{3}=\left(\begin{array}{cccc}
1 & 0 & \ldots & 0 \\
0 & -1 & \ldots & 0 \\
\ldots & \ldots & \ldots & \ldots \\
0 & 0 & \ldots & 0
\end{array}\right)_{N \times N}
\end{aligned}
$$

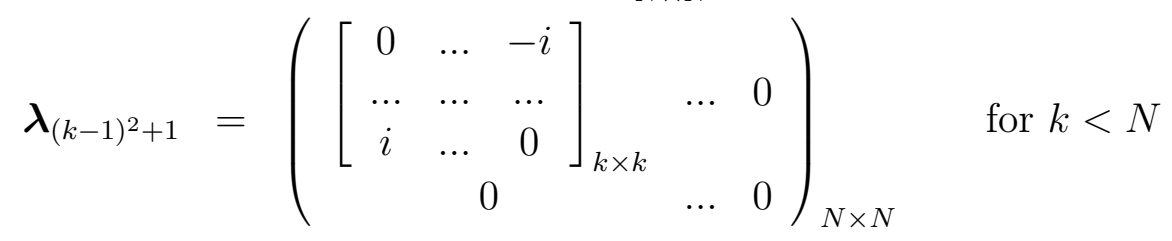

$$
\begin{aligned}
& \boldsymbol{\lambda}_{(N-1)^{2}+1}=\left(\begin{array}{ccc}
0 & \ldots & -i \\
\ldots & \ldots & \ldots \\
i & \ldots & 0
\end{array}\right)_{N \times N} \quad \text { for } k=N \\
& \boldsymbol{\lambda}_{N^{2}-1}=\sqrt{\frac{2}{N^{2}-N}}\left(\begin{array}{cccc}
1 & 0 & \ldots & 0 \\
0 & 1 & \ldots & 0 \\
\ldots & \ldots & \ldots & \ldots \\
0 & 0 & \ldots & -(N-1)
\end{array}\right)_{N \times N}
\end{aligned}
$$

This method provides a recipe to parametrize the natural orbitals of an $N \times N$ one-particle reduced density matrix, in terms of $N^{2}-1$ real parameters $\left\{\alpha_{i}\right\}_{i=1}^{N^{2}-1}$. The constraints on occupations $0<f_{n}<1$ can be fulfilled by parametrizing the 
occupations in terms of real parameters $x_{n}$ as,

$$
f_{n}=\frac{1}{2}\left[1-\cos \left(x_{n}\right)\right]
$$

where $x_{n}$ is a free parameter. Therefore we can write the grand-canonical potential in Eq. (B.1) as an unconstrained optimization in space of real parameters $\left\{\alpha_{i}\right\}_{i=1}^{N^{2}-1}$ and $\left\{x_{i}\right\}_{i=1}^{N}$.

The derivatives of the energy functional are generally available with respect to occupations $\left\{f_{i}\right\}_{i=1}^{N}$ and the natural orbitals i.e. $\left\{U_{i j}\right\}_{i, j=1}^{N}$. The unconstrained minimization orequires derivatives with respect to real parameters $\left\{\alpha_{i}\right\}_{i=1}^{N^{2}-1}$ and $\left\{x_{i}\right\}_{i=1}^{N}$. The energy functional derivatives are transformed as,

$$
\begin{aligned}
\frac{\partial E}{\partial x_{n}} & =\frac{\sin \left(x_{n}\right)}{2} \frac{\partial E}{\partial f_{n}} \\
\frac{\partial E}{\partial \alpha_{n}} & =\operatorname{Tr}\left(\frac{\partial E}{\partial \boldsymbol{U}} \frac{\partial \boldsymbol{U}}{\partial \alpha_{n}}+\frac{\partial E}{\partial \boldsymbol{U}^{\dagger}} \frac{\partial \boldsymbol{U}^{\dagger}}{\partial \alpha_{n}}\right)
\end{aligned}
$$

where the matrix $\frac{\partial \boldsymbol{U}}{\partial \alpha_{n}}$ is obtained by systematic differentiation of $\boldsymbol{U}$ in Eq. (B.3) with respect to $\alpha_{n}$ which requires inserting corresponding $i \boldsymbol{\lambda}$ matrix at the $N$-th position in the product of Eq. (B.3). 



\section{Grand potential and the Luttinger Ward functional}

In order to keep the discussion self-contained we discuss the perturbative expansion of the grand-canonical potential. This discussion can be found in any standard book of the many-body physics. We however closely follow along the lines of [9]. The ultimate goal of this section is to highlight the non-trivial relation between the grand-canonical potential and the Luttinger-Ward functional [70].

The grand-canonical potential for the Hamiltonian $\hat{\mathcal{H}}=\hat{\boldsymbol{h}}+\hat{\boldsymbol{W}}$ is defined as,

$$
\begin{aligned}
\Omega_{\beta}^{\hat{\boldsymbol{h}}+\hat{\boldsymbol{W}}} & =-k_{B} T \ln \mathcal{Z} \\
\mathcal{Z} & =\operatorname{Tr}\left(e^{-\beta(\hat{\boldsymbol{h}}+\hat{\boldsymbol{W}}-\mu \mathcal{N})}\right)
\end{aligned}
$$

For simplicity of the notation we defined two Hamiltonians $\hat{K}$ and $\hat{K}_{0}$ as,

$$
\begin{aligned}
\hat{K}_{0} & =\hat{\boldsymbol{h}}-\mu \mathcal{N} \\
\hat{K} & =\hat{\boldsymbol{h}}+\hat{\boldsymbol{W}}-\mu \mathcal{N}
\end{aligned}
$$

In the interaction picture the time evolution operator $\hat{U}(\tau)$ for the full Hamiltonian $\hat{K}$ has the form,

$$
\hat{U}(\tau)=e^{\tau \hat{K}_{0}} e^{-\tau \hat{K}}
$$

where the equation of motion of the $\hat{U}(\tau)$ and its formal solution has the form,

$$
\begin{aligned}
\frac{d \hat{U}(\tau)}{d \tau} & =-\hat{\boldsymbol{W}}(\tau) \hat{U}(\tau) \\
\hat{U}(\tau) & =\sum_{n} \frac{(-1)^{n}}{n !} \int_{0}^{\tau} d \tau_{1} \int_{0}^{\tau} d \tau_{2} \ldots \int_{0}^{\tau} d \tau_{n} \mathcal{T}_{\tau}\left(\hat{\boldsymbol{W}}\left(\tau_{1}\right) \hat{\boldsymbol{W}}\left(\tau_{2}\right) \ldots \hat{\boldsymbol{W}}\left(\tau_{n}\right)\right) \\
& =\mathcal{T}_{\tau} \exp \left(-\int_{0}^{\tau} d \tau^{\prime} \hat{\boldsymbol{W}}\left(\tau^{\prime}\right)\right)
\end{aligned}
$$

where the time ordering operator $\mathcal{T}_{\tau}$ orders the operator in decreasing order of time $\tau$. The partition function $\mathcal{Z}$ can be written in terms of time evolution operator $\hat{U}(\tau)$ evaluated at imaginary time $\tau=\beta$, as

$$
\mathcal{Z}=\operatorname{Tr}\left(e^{-\beta \hat{K}_{0}} \hat{U}(\beta)\right)
$$




$$
=\operatorname{Tr}\left(e^{-\beta \hat{K}_{0}}\right)\langle\hat{U}(\beta)\rangle_{0}
$$

where the average $\langle\hat{U}(\beta)\rangle_{0}$ is taken with the non-interacting density-matrix $\mathrm{e}^{-\beta \hat{K}_{0}}$, corresponding to the Hamiltonian $\hat{K}_{0}$ as,

$$
\begin{aligned}
&\langle\hat{U}(\beta)\rangle_{0}=\frac{\operatorname{Tr}\left(e^{-\beta \hat{K}_{0}} \hat{U}(\beta)\right)}{\operatorname{Tr}\left(e^{-\beta \hat{K}_{0}}\right)} \\
&=\sum_{n} \frac{(-1)^{n}}{n !} \int_{0}^{\tau} d \tau_{1} \int_{0}^{\tau} d \tau_{2} \ldots \int_{0}^{\tau} d \tau_{n}\left\langle\mathcal{T}_{\tau}\left(\hat{\boldsymbol{W}}\left(\tau_{1}\right) \hat{\boldsymbol{W}}\left(\tau_{2}\right) \ldots \hat{\boldsymbol{W}}\left(\tau_{n}\right)\right)\right\rangle_{0} \\
&=\sum_{n} \frac{(-1)^{n}}{2^{n} n !} \sum_{\alpha_{1} \beta_{1} \gamma_{1} \delta_{1}} \sum_{\alpha_{2} \beta_{2} \gamma_{2} \delta_{2}} \ldots \sum_{\alpha_{n} \beta_{n} \gamma_{n} \delta_{n}} W_{\alpha_{1} \beta_{1} \gamma_{1} \delta_{1}} W_{\alpha_{2} \beta_{2} \gamma_{2} \delta_{2}} \ldots W_{\alpha_{n} \beta_{n} \gamma_{n} \delta_{n}} \\
& \int_{0}^{\tau} d \tau_{1} \int_{0}^{\tau} d \tau_{2} \ldots \int_{0}^{\tau} d \tau_{n}\left\langle\mathcal{T}_{\tau}\left(\hat{c}_{\alpha_{1}}^{\dagger}\left(\tau_{1}\right) \hat{c}_{\beta_{1}}^{\dagger}\left(\tau_{1}\right) \hat{c}_{\delta_{1}}\left(\tau_{1}\right) \hat{c}_{\gamma_{1}}\left(\tau_{1}\right) \ldots \hat{c}_{\alpha_{n}}^{\dagger}\left(\tau_{n}\right) \hat{c}_{\beta_{n}}^{\dagger}\left(\tau_{n}\right) \hat{c}_{\delta_{n}}\left(\tau_{n}\right) \hat{c}_{\gamma_{n}}\left(\tau_{n}\right)\right)\right\rangle_{0}
\end{aligned}
$$

The average with respect to non-interacting density matrix $\mathrm{e}^{-\beta \hat{K}_{0}}$, leads to simplicity for evaluating terms order by order in the expansion of $\mathcal{Z}$ in Eq. (C.6). The simplicity comes from the fact that one can use Wick's theorem to evaluate each n-th order term, which simply demands enumeration of all the contractions possible. The contraction means pairing of all the annihilations with creation operators. Each contraction corresponds to a non-interacting Green's function. The n-th order term in Eq. C.6), has $2 n$ creation and annihilation operators each. Therefore a total of $(2 n)$ ! contractions has to be evaluated for an n-th order term. Each of the $(2 n)$ ! terms are represented by distinct diagrams $D \in S_{n}$. The partition function $\mathcal{Z}$ can be expressed in terms of the diagrams as,

$$
\mathcal{Z}=\operatorname{Tr}\left(e^{-\beta \hat{K}_{0}}\right) \sum_{n} \sum_{D \in S_{n}} \frac{1}{2^{n} n !} V(D)
$$

where the mathematical expression $V(D)$ of the diagram $D$, can be obtained by following a set of rules [9] which will be sketched in the following section.

\section{C.1 Rules to evaluate the diagrams}

The interaction vertex $W_{\alpha \beta, \gamma \delta}$ is given by a wavy line with two incoming and two outgoing lines. The outgoing lines corresponds to the creation operators $\hat{c}_{\alpha}^{\dagger}$ and $\hat{c}_{\beta}^{\dagger}$ whereas the incoming lines corresponds to annihilation operators $\hat{c}_{\gamma}$ and $\hat{c}_{\delta}$

The contraction corresponds to pairing of a creation operator $\hat{c}_{\alpha_{i}}^{\dagger}\left(\tau_{i}\right)$ from one interaction vertex, with an annihilation operator $\hat{c}_{\alpha_{j}}\left(\tau_{j}\right)$ of the same or another interaction vertex. This contraction corresponds to a Green's function $\mathcal{G}_{\alpha_{j} \alpha_{i}}^{0}\left(\tau_{j}-\right.$ 
$\left.\tau_{i}\right)$ represented by a solid directed line with arrow originating from index $\alpha_{i}$ and terminating at the index $\alpha_{j}$.

The fourier transformation of the Green's function as defined in Eq. (2.50), allows the time integrals in Eq. C.6) to be performed easily. Since for any interaction vertex there are two incoming and two outgoing Green's function lines and each of these four Green's function will have $\tau$ in common. Therefore the dependence on $\tau$ for any diagram will be of the form,

$$
\exp \left(\tau\left(i \omega_{n_{1}}+i \omega_{n_{2}}-i \omega_{n_{3}}-i \omega_{n_{4}}\right)\right)
$$

which when integrated over the range $[0, \beta]$ leads to a matsubara frequency conservation rule,

$$
i \omega_{n_{1}}+i \omega_{n_{2}}=i \omega_{n_{3}}+i \omega_{n_{4}}
$$

There the matsubara frequencies at each interaction vertex should be conserved, i.e. the total frequency associated with Green's function entering a particular vertex should be equal to the total frequency associated with outgoing Green's function lines.

To sum up all the observations, one sets up a list of rules [9] to evaluate a particluar diagram, which are listed below.

The evaluation of partition function $\mathcal{Z}$ in Eq.(C.7) starts with drawing all distinct diagrams with n-vertices connected by directed lines. Two diagrams are distinct, if they cannot be deformed so as to coincide completely. The value $V(D)$ of each diagram $D$ in a set $S_{n}$ of $(2 n)$ ! distinct diagrams is evaluated using following rules,

- For each interaction vertex include,

$$
W_{\alpha \beta, \gamma \delta}=\gamma_{\gamma}^{\alpha}-\cdots
$$

- Assign a single particle non-interacting Green's function $\mathcal{G}_{\alpha_{j} \alpha_{i}}^{0}\left(i \omega_{n}\right)$ to a directed line as,

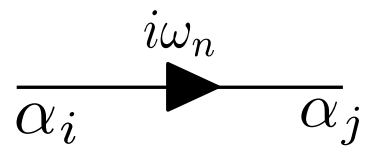

- Restrict the number of matsubara frequecies $i \omega_{n}$, by the conservation theorem of Eq.(C.9). This in general limits the number of independent matsubara frequencies. For an $n$-th order diagram, there are $n+1$ independent matsubara frequencies.

- Green's function lines originating and terminating at the same interaction vertex leads to an extra factor of $e^{i \omega_{n} 0^{+}}$. 
- For each fermionic loop, there is a sign factor of -1 . Therefore include an overall factor of $\frac{(-1)^{n+n_{F}}}{\beta^{n}}$ where $n_{F}$ is the number of fermi loops or closed paths in the diagram.

- Sum over all the orbitals indices $\alpha^{\prime} s$ and the independent matsubara frequencies.

\section{C.2 Symmetry factors of the diagrams}

\section{This section summarizes the discussion about symmetry factors in [9]}

The brute-force way of evaluating diagrams is practically impossible, since the number of diagrams grow dramatically with the order $n$. For example for the order $n=3$, there are total of $6 !=720$ diagrams. Therefore we need some additional simplification to efficiently account for all the diagrams at a particular order $n$ of perturbation. It was realized that several distinct diagrams $D \in S_{n}$ have same numerical value $V(D)$.

To this direction we consider set of transformations on a diagram $D \in S_{n}$ which leaves the value $V(D)$ of the diagram $D$ invariant. For a general interaction vertex with the property

$$
W_{\alpha \beta, \gamma \delta}=W_{\beta \alpha, \delta \gamma}
$$

there are two types of transformations which leave the value of diagrams invariant.

- Since all the time label $\tau_{1}, \tau_{2} \ldots \tau_{n}$ are integrated over, therefore any permutation of time variables leave the value of diagram invariant. So for $n$ interaction vertex, there are $n$ ! number of such transformations available.

- Exchange of extremities of the interaction vertex also keeps the value of diagram invariant, since all the indices of interactions are summed up and the interchanging the extremities does not change the value of interaction as shown in Eq. C.10.

Therefore for each diagram of $n$-th order, there are $2^{n} n$ ! transformations available which leave the value of diagram unchanged. Now we consider the action of these transformations on a diagram $D \in S_{n}$. Some transformations will transform the diagram $D$ in a deformation of itself while the rest of transformations will yield a different diagram but with the same numerical value.

We introduce a symmetry factor of diagram $D$ as $S(D)$ which will be the number of deformations produced by the transformations. Therefore the number of topologically equivalent diagrams are $\frac{2^{n} n !}{S(D)}$. Therefore the partition function can be rewritten 
in terms of topologically distinct diagrams as,

$$
\mathcal{Z}=\operatorname{Tr}\left(e^{-\beta \hat{K}_{0}}\right) \sum_{n} \sum_{D \in T_{n}} \frac{1}{S(D)} V(D)
$$

where $T_{n}$ is the set of topologically distinct diagrams with $n$ interaction vertices.

\section{C.3 Linked cluster theorem}

The evaluation of the grand potential $\Omega_{\beta}^{\hat{h}+\hat{W}}$ requires taking the logarithm of the partition function $\mathcal{Z}$ and has the form,

$$
\begin{aligned}
\Omega_{\beta}^{\hat{h}+\hat{W}} & =\Omega_{\beta}^{0}-\frac{1}{\beta} \ln \left(\sum_{n} \sum_{D \in S_{n}} \frac{1}{2^{n} n !} V(D)\right) \\
\Omega_{\beta}^{0} & =-\frac{1}{\beta} \ln \left[\operatorname{Tr}\left(e^{-\beta \hat{K}_{0}}\right)\right]
\end{aligned}
$$

The set $S_{n}$ in Eq. C.12) also contain diagrams which have disconnected parts that are not connected to other part of the diagram through Green's function line or the interaction vertex. The value $V(D)$ of diagram $D \in S_{n}$ which has say two disconnected pieces $D_{a}$ and $D_{b}$ with $n_{a}$ and $n_{b}$ interaction vertices respectively such that $n=n_{a}+n_{b}$, can be written as,

$$
V\left(D=D_{a}+D_{b}\right)=V\left(D_{a}\right) V\left(D_{b}\right)
$$

The linked cluster theorem enables us to write the grand potential in terms of only distinct connected diagrams $([9])$ as,

$$
\Omega_{\beta}^{\hat{\boldsymbol{h}}+\hat{\boldsymbol{W}}}=\Omega_{\beta}^{0}-\frac{1}{\beta} \ln \left(\sum_{n} \sum_{D \in C_{n}} \frac{V(D)}{S(D)}\right)
$$

where $C_{n}$ is the set of topologically distinct connected diagrams with $n$ interaction vertices.

\section{C.4 Luttinger Ward functional $\Phi^{\hat{W}}[\boldsymbol{G}]$}

Luttinger and Ward in their seminal work [70], derived an expression for the grand canonical potential in terms of a universal Green's function functional $\Phi^{\hat{\boldsymbol{W}}}[\boldsymbol{G}]$. They 
defined a quantity $Y$ as,

$$
Y=-\frac{1}{\beta} \sum_{\omega_{n}} \operatorname{Tr}\left\{\ln \left(-\boldsymbol{G}^{-1}\right)+\Sigma\left(i \omega_{n}\right) \boldsymbol{G}\left(i \omega_{n}\right)\right\}+\Phi^{\hat{\boldsymbol{W}}}[\boldsymbol{G}]
$$

where $\boldsymbol{G}^{-1}$ is the interacting Green's function and $\Sigma$ is the self-energy defined in the previous section. The trace of the logarithm of matrix $\boldsymbol{G}^{-1}$ is obtained by summing up the logarithm of eigen-values of $\boldsymbol{G}^{-1}$. The Luttinger Ward functional $\Phi^{\hat{\boldsymbol{W}}}[\boldsymbol{G}]$ is a Green's function functional which is constructed as a sum of infinite set of closed, connected skeleton Feynman diagrams. The skeleton diagrams are obtained by removing the self-energy insertions in the respective diagram. In diagramatic language, it means that by cutting any two Green's function lines, the diagram does not decompose into sub-diagrams.

In terms of diagrams the Luttinger Ward functional $\Phi^{\hat{\boldsymbol{W}}}[\boldsymbol{G}]$ has the following form,

$$
\Phi^{\hat{\boldsymbol{W}}}[\boldsymbol{G}]=-\frac{1}{\beta} \sum_{n} \sum_{D \in C_{n}^{s}} \frac{V(D)}{S(D)}
$$

where $C_{n}^{s}$ is the set of skeleton diagrams having $n$ interaction vertices.

The Luttinger Ward functional $\Phi^{\hat{W}}[G]$ by its construction follows certain important properties,

$$
\begin{aligned}
\Phi^{\hat{\boldsymbol{W}=0}}[\boldsymbol{G}] & =0 \\
\frac{\delta \Phi^{\hat{\boldsymbol{W}}}[\boldsymbol{G}]}{\delta G_{\alpha \beta}\left(i \omega_{n}\right)} & =\frac{1}{\beta} \Sigma_{\beta \alpha}\left(i \omega_{n}\right)
\end{aligned}
$$

where the first property in Eq. C.17) states that the Luttinger Ward functional $\Phi^{\hat{\boldsymbol{W}}}[\boldsymbol{G}]$ is a universal functional that depends only on the interaction between the electrons and not on the external potential. The second property states that the first order variation of the Luttinger Ward functional $\Phi^{\hat{W}}[\boldsymbol{G}]$ with respect to the Green's function $\boldsymbol{G}$ provides the self-energy of the interacting fermions.

Using the following properties of $\Phi^{\hat{W}}[\boldsymbol{G}]$, Luttinger and Ward proved that the quantity $Y$ in Eq. C.15 is equal to the grand potential $\Omega_{\beta}^{\hat{h}+\hat{W}}$ and is stationary with respect to the self-energy i.e.,

$$
\begin{aligned}
Y & =\Omega_{\beta}^{\hat{h}+\hat{W}} \\
\frac{\partial Y}{\partial \Sigma_{\alpha \beta}\left(i \omega_{n}\right)} & =0
\end{aligned}
$$


The stationarity condition in Eq. C.18), leads to the celebrated Dyson equation,

$$
\boldsymbol{G}\left(i \omega_{n}\right)=\frac{\mathbf{1}}{\left(i \omega_{n}+\mu\right) \mathbf{1}-\boldsymbol{h}-\boldsymbol{\Sigma}\left(i \omega_{n}\right)}
$$

The more elaborate proof of Luttinger-Ward identity can be found in lecture notes of Robert Eder [107]. 



\section{Bibliography}

[1] M. Born and R. Oppenheimer. Zur quantentheorie der molekeln. Annalen der Physik, 389(20):457-484, 1927.

[2] Pierre Hohenberg and Walter Kohn. Inhomogeneous electron gas. Phys. Rev., 136:B864, 1964.

[3] Walter Kohn and Lu J. Sham. Self-consistent equations including exchange and correlation effects. Phys. Rev., 140:A1133, 1965.

[4] Robert O. Jones and Olle Gunnarsson. The density functional formalism, its applications and prospects. Rev. Mod. Phys., 61:689, 1989.

[5] R.O. Jones. Density functional theory: Past, present, ... future? PsiK Newsletter, 124:0, 2015.

[6] T. L. Gilbert. Hohenberg-Kohn theorem for nonlocal external potentials. Phys. Rev. B, 12:2111, 1975.

[7] M. Levy. Universal variational functionals of electron densities, first order density matrixes and natural spin-orbitals and solution of the v-representability problem. Proc. Nat'l Acad. Sci. USA, 76:6062, 1979.

[8] G.D. Mahan. Many-Particle Physics. Physics of Solids and Liquids. Springer, 2000 .

[9] J.W. Negele and H. Orland. Quantum many-particle systems. Frontiers in physics. Addison-Wesley Pub. Co., 1988.

[10] Walter Metzner and Dieter Vollhardt. Correlated lattice fermions in $d=\infty$ dimensions. Phys. Rev. Lett., 62:324-327, Jan 1989.

[11] E. Müller-Hartmann. Correlated fermions on a lattice in high dimensions. $Z$. Phys. B, 74:507-512, 1989.

[12] Antoine Georges, Gabriel Kotliar, Werner Krauth, and Marcelo J. Rozenberg. Dynamical mean-field theory of strongly correlated fermion systems and the limit of infinite dimensions. Rev. Mod. Phys., 68:13-125, Jan 1996. 
[13] Christopher J. Cramer and Donald G. Truhlar. Density functional theory for transition metals and transition metal chemistry. Phys. Chem. Chem. Phys., 11:10757-10816, 2009.

[14] D.C. Langreth and M.J. Mehl. Beyond the local-density approximation in calculations of ground-state electronic properties. Phys. Rev. B, 28:1809, 1983.

[15] H. Bahmann and M. Ernzerhof. Generalized-gradient exchange-correlation hole obtained from a correlation factor ansatz. J. Chem. Phys., 128:234104, 2008 .

[16] John P. Perdew, Kieron Burke, and Matthias Ernzerhof. Generalized gradient approximation made simple. Phys. Rev. Lett, 77:3865, 1996.

[17] J.P. Perdew, K. Burke, and M. Ernzerhof. Erratum: Generalized gradient approximation made simple. Phys. Rev. Lett, 78:1396, 1997.

[18] A. D. Becke. Density-functional thermochemistry. I. the effect of the exchangeonly gradient correction. J. Chem. Phys., 96:2155, 1992.

[19] A. D. Becke. Density-functional thermochemistry. II. the effect of the perdewwang generalized-gradient correlation correction. J. Chem. Phys., 97:9173, 1992 .

[20] Axel D. Becke. Density functional thermochemistry. III. the role of exact exchange. J. Chem. Phys, 98:5648, 1993.

[21] Axel D. Becke. Density-functional thermochemistry. IV. a new dynamical correlation functional and implications for exact-exchange mixing. J. Chem. Phys., 104:1040, 1996.

[22] Axel D. Becke. Density-functional thermochemistry. V. systematic optimization of exchange-correlation functionals. J. Chem. Phys., 107:8554, 1997.

[23] Axel D. Becke. A new mixing of hartree-fock and local density-functional theories. J. Chem. Phys., 98:1372, 1993.

[24] P.J. Stephens, F.J. Devlin, C.F. Chabalowski, and M.J. Frisch. Ab-initio calculation of vibrational adsorption and circular dichroism spectra using densityfunctional force fields. J. Phys. Chem, 98:11623, 1994.

[25] M. Ernzerhof and G.E. Scuseria. Assessment of the Perdew-Ernzerhof exchange-correlation functional. J. Chem. Phys., 110:5029, 99.

[26] C. Adamo and V. Barone. Toward reliable density functional methods without adjustable parameters: The PBE0 model. J. Chem. Phys., 110:6158, 1999. 
[27] Ulf von Barth. Basic density-functional theory: An overview. Physica Scripta, 2004(T109):9, 2004.

[28] Aron J. Cohen, Paula Mori-Sánchez, and Weitao Yang. Challenges for density functional theory. Chem. Rev., 112(1):289, 2012.

[29] K. Terakura, T. Oguchi, A. R. Williams, and J. Kübler. Band theory of insulating transition-metal monoxides: Band-structure calculations. Phys. Rev. $B, 30: 4734-4747$, Oct 1984 .

[30] A. Georges and G. Kotliar. Hubbard model in infinite dimensions. Phys. Rev. $B, 45: 6479,1992$.

[31] M. Imada, A. Fujimori, and Y. Tokura. Metal-insulator transitions. Rev. Mod. Phys., 70:1039, 1998.

[32] Karsten Held, Igor A. Nekrasov, Georg Keller, Volker Eyert, Nils Blümer, Andrew K. McMahan, Richard T. Scalettar, Thomas Pruschke, Vladimir I. Anisimov, and Dieter Vollhardt. The LDA+DMFT approach to materials with strong electronic correlations. NIC Series (John von Neumann Institute for Computing), 10:175, 2002.

[33] K. Held and. I.A. Nekrasov, G. Keller, V. Eyert, N. Blümer, A.K. McMahan, R.T. Scalettar, Th. Pruschke, V.I. Anisimov, and D. Vollhardt. Realistic investigations of correlated electron systems with LDA+DMFT. PsiK Newsletter, $56: 65,2003$.

[34] K. Held. Electronic structure calculations using dynamical mean field theory. Adv. Phys., 56(6):829-926, 2007.

[35] G. Kotliar, S. Y. Savrasov, K. Haule, V. S. Oudovenko, O. Parcollet, and C. A. Marianetti. Electronic structure calculations with dynamical mean-field theory. Rev. Mod. Phys., 78:865-951, Aug 2006.

[36] Vladimir I. Anisimov, Jan Zaanen, and Ole K. Andersen. Band theory and Mott insulators: Hubbard $U$ instead of Stoner I. Phys. Rev. B, 44:943-954, Jul 1991.

[37] Vladimir I. Anisimov, F. Aryasetiawan, and A. I. Lichtenstein. First-principles calculations of the electronic structure and spectra of strongly correlated systems: the LDA+U method. J. Phys.: Condens. Matter, 9:767, 1997.

[38] B. Amadon, F. Jollet, and M. Torrent. $\gamma$ and $\beta$ Cerium: LDA $+\mathrm{U}$ calculations of ground-state parameters. Phys. Rev. B, 77:155104, 2008. 
[39] D.A. Andersson, S.I. Simak, B. Johansson, I.A. Abrikosov, and N. V. Skorodumova. Modeling of $\mathrm{CeO}_{2}, \mathrm{Ce}_{2} \mathrm{O}_{3}$, and $\mathrm{CeO}_{2-x}$ in the $\mathrm{LDA}+\mathrm{U}$ formalism. Phys. Rev. B, 75:35109, 2007.

[40] J. Bünemann, W. Weber, and F. Gebhard. Multiband gutzwiller wave functions for general on-site interactions. Phys. Rev. B, 57:6896-6916, Mar 1998.

[41] Tobias Schickling, Jörg Bünemann, Florian Gebhard, and Werner Weber. Gutzwiller density functional theory: a formal derivation and application to ferromagnetic nickel. New J. Phys., 16:83034, 2014.

[42] Guang-Tao Wang, Xi Dai, and Zhong Fang. Phase diagram of $\mathrm{Na}_{x} \mathrm{CoO}_{2}$ studied by Gutzwiller density-functional theory. Phys. Rev. Lett., 101:066403, Aug 2008.

[43] Ruanchen Dong, Xiangang Wan, Xi Dai, and Sergey Y. Savrasov. Orbitaldependent electronic masses in Ce heavy-fermion materials studied via gutzwiller density-functional theory. Phys. Rev. B, 89:165122, Apr 2014.

[44] Martin C. Gutzwiller. Effect of correlation on the ferromagnetism of transition metals. Phys. Rev. Lett., 10(5):159, 1963.

[45] J. Hubbard. Electron correlations in narrow energy bands. Proc. R. Soc. Lond. A, 276:238, 1963.

[46] J. Kanamori. Electron correlation and ferromagnetism of transition metals. Prog. Theor. Phys., 30:275, 1963.

[47] P. E. Blöchl, C. F. J. Walther, and Th. Pruschke. Method to include explicit correlations into density-functional calculations based on density-matrix functional theory. Phys. Rev. B, 84:205101, 2011.

[48] Peter E. Blöchl, Thomas Pruschke, and Michael Potthoff. Density-matrix functionals from green's functions. Phys. Rev. B, 88:205139, Nov 2013.

[49] J. M. Luttinger and J. C. Ward. Ground-state energy of a many-fermion system ii. Phys. Rev., 118:1417, 1960.

[50] A. M. K. Müller. Explicit approximation between reduced two- and oneparticle density matrices. Phys. Lett., 105:446, 1984.

[51] Oleg Gritsenko, Katarzyna Pernal, and Evert Jan Baerends. An improved density matrix functional by physically motivated repulsive corrections. $J$. Chem. Phys., 122(20):-, 2005. 
[52] S. Goedecker and C. J. Umrigar. Natural orbital functional for the manyelectron problem. Phys. Rev. Lett., 81:866-869, Jul 1998.

[53] Sangeeta Sharma, J. Kay Dewhurst, Nektarios N. Lathiotakis, and Eberhard K. U. Gross. Reduced density matrix functional for many-electron systems. Phys. Rev. B, 78:201103, 2008.

[54] Miguel A. L. Marques and N. N. Lathiotakis. Empirical functionals for reduced-density-matrix-functional theory. Phys. Rev. A, 77:032509, Mar 2008.

[55] D J Carrascal, J Ferrer, J C Smith, and K Burke. The hubbard dimer: a density functional case study of a many-body problem. Journal of Physics: Condensed Matter, 27(39):393001, 2015.

[56] Ebad Kamil, Robert Schade, Thomas Pruschke, and Peter E. Blöchl. Reduced density-matrix functionals applied to the hubbard dimer. Phys. Rev. B, 93:085141, Feb 2016.

[57] Roberto Car and Michele Parrinello. Unified approach for molecular dynamics and density-functional theory. Phys. Rev. Lett, 55:2471, 1985.

[58] R. Car and M. Parrinello. Simple Molecular Systems at Very High Density, chapter A Unified Approach for Molecular Dynamics and Density Functional Theory, page 455. Plenum, NY, 1989.

[59] M. Born and K. Huang, editors. Dynamical Theory of Crystal Lattices. Oxford Clarendon Press, 1954.

[60] P. Blöchl. The electronic structure of matter. Advanced Topics of Theoretical Physics 1. 2013.

[61] J. von Neumann. Wahrscheinlichkeitstheoretischer aufbau der quantenmechanik. Nachrichten von der Gesellschaft der Wissenschaften zu Göttingen, Mathematisch-Physikalische Klasse, 1927:245-272, 1927.

[62] A. J. Coleman. Structure of fermion density matrices. Rev. Mod. Phys., 35:668-686, Jul 1963.

[63] A.J. Coleman. Discussion on "structure of fermion density matrices". Rev. Mod. Phys., 35:687, 1963.

[64] Per-Olov Löwdin. Quantum theory of many-particle systems. i. physical interpretations by means of density matrices, natural spin-orbitals, and convergence problems in the method of configurational interaction. Phys. Rev., 97:1474-1489, Mar 1955. 
[65] P. Blöchl. Theory of first principle calculations. CP-PAW Hands-On Course on First-Principles Calculations. 2015.

[66] T. Matsubara. A new approach to quantum-statistical physics. Prog. Theor. Phys., 14:351, 1955.

[67] M. Levy. Universal Variational Functionals of Electron Densities, FirstOrder Density Matrices, and Natural Spin-Orbitals and Solution of the vRepresentability Problem. Proceedings of the National Academy of Science, 76:6062-6065, December 1979.

[68] Peter E. Blöchl, Christian F. J. Walther, and Thomas Pruschke. Method to include explicit correlations into density-functional calculations based on density-matrix functional theory. Phys. Rev. B, 84:205101, Nov 2011.

[69] Jean-Paul Ryckaert, Giovanni Ciccotti, and Herman J. C. Berendsen. Numerical integration of cartesian equations of motion of a system with constraints: molecular dynamics of alkenes. J. Comput. Phys., 23:327, 1977.

[70] W. Kohn and J. M. Luttinger. Ground-state energy of a many-fermion system. Phys. Rev., 118:41-45, Apr 1960.

[71] E. J. Baerends. Exact exchange-correlation treatment of dissociated $\mathrm{H}_{2}$ in density functional theory. Phys. Rev. Lett, 87:133004, 2001.

[72] M.A. Buijse and E.J. Baerends. An approximate exchange-correlation hole density as a functional of the natural orbitals. Mol. Phys., 100:401, 2002.

[73] John P. Perdew, Kieron Burke, and Yue Wang. Generalized gradient approximation for the exchange-correlation hole of a many-electron system. Phys. Rev. B, 54:16533-16539, Dec 1996.

[74] Jerzy Cioslowski and Katarzyna Pernal. Constraints upon natural spin orbital functionals imposed by properties of a homogeneous electron gas. The Journal of Chemical Physics, 111(8), 1999.

[75] Gábor Csányi and T. A. Arias. Tensor product expansions for correlation in quantum many-body systems. Phys. Rev. B, 61:7348-7352, Mar 2000.

[76] N. N. Lathiotakis, N. Helbig, and E. K. U. Gross. Performance of one-body reduced density-matrix functionals for the homogeneous electron gas. Phys. Rev. B, 75:195120, May 2007.

[77] N. N. Lathiotakis and Miguel A. L. Marques. Benchmark calculations for reduced density-matrix functional theory. The Journal of Chemical Physics, 128(18), 2008. 
[78] Mario Piris. A new approach for the two-electron cumulant in natural orbital functional theory. International Journal of Quantum Chemistry, 106(5):10931104, 2006.

[79] N. N. Lathiotakis, S. Sharma, J. K. Dewhurst, F. G. Eich, M. A. L. Marques, and E. K. U. Gross. Density-matrix-power functional: Performance for finite systems and the homogeneous electron gas. Phys. Rev. A, 79:040501, Apr 2009 .

[80] S. Sharma, J. K. Dewhurst, S. Shallcross, and E. K. U. Gross. Spectral density and metal-insulator phase transition in mott insulators within reduced density matrix functional theory. Phys. Rev. Lett., 110:116403, Mar 2013.

[81] Yosuke Nagaoka. Ferromagnetism in a narrow, almost half-filled $s$ band. Phys. Rev., 147:392-405, Jul 1966.

[82] Philip W. Anderson. Theory of magnetic exchange interactions:exchange in insulators and semiconductors. volume 14 of Solid State Physics, pages $99-$ 214. Academic Press, 1963.

[83] P. W. ANDERSON. The resonating valence bond state in la2cuo4 and superconductivity. Science, 235(4793):1196-1198, 1987.

[84] F. C. Zhang and T. M. Rice. Effective hamiltonian for the superconducting cu oxides. Phys. Rev. B, 37:3759-3761, Mar 1988.

[85] Elliott H. Lieb and F. Y. Wu. Absence of mott transition in an exact solution of the short-range, one-band model in one dimension. Phys. Rev. Lett., 20:14451448, Jun 1968.

[86] Elliott H. Lieb and F.Y. Wu. The one-dimensional hubbard model: a reminiscence. Physica A: Statistical Mechanics and its Applications, 321(1-2):1-27, 2003. Statphys-Taiwan-2002: Lattice Models and Complex Systems.

[87] M. P. Vecchi S. Kirkpatrick, C. D. Gelatt. Optimization by simulated annealing. Science, 220(4598):671-680, 1983.

[88] Loup Verlet. Computer "experiments" on classical fluids. i. thermodynamical properties of lennard-jones molecules. Phys. Rev., 159:98-103, Jul 1967.

[89] N. Helbig, N. N. Lathiotakis, and E. K. U. Gross. Discontinuity of the chemical potential in reduced-density-matrix-functional theory for open-shell systems. Phys. Rev. A, 79:022504, Feb 2009. 
[90] N. N. Lathiotakis, S. Sharma, N. Helbig, J.K.Dewhurst, M.A.L.Marques, F. Eich, T. Baldsiefen, A. Zacarias, and E. K. U. Gross. Discontinuities of the chemical potential in reduced density matrix functional theory. Zeitschrift fuer Physikalische Chemie, 224:467-480, 2010.

[91] N. Helbig, N. N. Lathiotakis, M. Albrecht, and E. K. U. Gross. Discontinuity of the chemical potential in reduced-density-matrix-functional theory. EPL (Europhysics Letters), 77(6):67003, 2007.

[92] Y. Shinohara, S. Sharma, S. Shallcross, N. N. Lathiotakis, and E. K. U. Gross. Spectrum for nonmagnetic mott insulators from power functional within reduced density matrix functional theory. Journal of Chemical Theory and Computation, 11(10):4895-4899, 2015. PMID: 26574277.

[93] Y Shinohara, S Sharma, J K Dewhurst, S Shallcross, N N Lathiotakis, and E K U Gross. Doping induced metal-insulator phase transition in nio. a reduced density matrix functional theory perspective. New Journal of Physics, 17(9):093038, 2015.

[94] John P. Perdew, Robert G. Parr, Mel Levy, and Jose L. Balduz. Densityfunctional theory for fractional particle number: Derivative discontinuities of the energy. Phys. Rev. Lett., 49:1691-1694, Dec 1982.

[95] S. Sharma, J. K. Dewhurst, N. N. Lathiotakis, and E. K. U. Gross. Reduced density matrix functional for many-electron systems. Phys. Rev. B, 78:201103, Nov 2008.

[96] Gordon Baym. Self-consistent approximations in many-body systems. Phys. Rev., 127:1391-1401, Aug 1962.

[97] Jan Gukelberger, Li Huang, and Philipp Werner. On the dangers of partial diagrammatic summations: Benchmarks for the two-dimensional hubbard model in the weak-coupling regime. Phys. Rev. B, 91:235114, Jun 2015.

[98] N. E. Bickers and D.J. Scalapino. Conserving approximations for strongly fluctuating electron systems. i. formalism and calculational approach. Ann. Phys., 193:206, 1989.

[99] N.E. Bickers and S.R. White. Conserving approximations for strongly fluctuating electron systems. ii. numerical results and parquet extension. Phys. Rev. B, 43:8044, 1991.

[100] N. E. Bickers, D. J. Scalapino, and S. R. White. Conserving approximations for strongly correlated electron systems: Bethe-salpeter equation and dynamics for the two-dimensional hubbard model. Phys. Rev. Lett., 62:961-964, Feb 1989. 
[101] Hu Wang, Shun-Li Yu, and Jian-Xin Li. Fermi arcs, pseudogap, and collective excitations in doped $\mathrm{sr}_{2} \mathrm{iro}_{4}$ : A generalized fluctuation exchange study. Phys. Rev. B, 91:165138, Apr 2015.

[102] M. Galitskii. The energy spectrum of a non-ideal fermi gas. Sov. Phys.-JETP, $7(1): 104,1958$.

[103] M. Galassi, J. Davies, J. Theiler, B. Gough, G. Jungman, P. Alken, M. Booth, and F. Rossi. GNU Scientific Library Reference Manual. Network Theory Ltd, 3rd edition, 2009. http://www.gnu.org/software/gsl/.

[104] Steven G. Johnson and M. J. D. Powell. The NLopt nonlinear-optimization package. http://ab-initio.mit.edu/nlopt.

[105] Todd Tilma, Mark Byrd, and E C G Sudarshan. A parametrization of bipartite systems based on SU(4) euler angles. Journal of Physics A: Mathematical and General, 35(48):10445, 2002.

[106] Todd Tilma and E C G Sudarshan. Generalized euler angle parametrization for SU(N). Journal of Physics A: Mathematical and General, 35(48):10467, 2002 .

[107] R. Eder. The variational cluster approximation. In Eva Pavarini, Erik Koch, and Ulrich Schollwöck, editors, Emergent Phenomena in Correlated Matter, volume 3 of Modeling and Simulation. Forschungszentrum Jülich Zentralbibliothek, Verlag, Sep 2013. 



\section{Acknowledgement}

Firstly, I would like to express my sincere gratitude to my advisors Prof. Dr. Thomas Pruschke (1959-2016) and Prof. Dr. Peter E. Blöchl for offering me a position to pursue my $\mathrm{PhD}$ study and research, for their motivation and patience. Their guidance and assistance throughout my work, helped me in successfully writing this thesis.

The administrative issues after the sad demise of Prof. Pruschke, were handled very efficiently by the secretaries, senior professors and the group leaders of the Condensed Matter Theory group at Instut für Theoretische Physik.

I would like to thank Prof. Dr. Stefan Kehrein for agreeing to be on my thesis committee and keeping a close track of my progress during the course of my PhD studies. Sincere thanks to Dr. Salvatore Manmana, Dr. Rainer Härtle, Dr. Maria Medvedyeva (currently in Slovenia), Dr. Davide Fioretto and Mr. Robert Schade for the fruitful discussions and insightful comments that motivated me to pursue my research work with zeal.

Sincere thanks to the group members for providing a dynamic and vibrant atmosphere for the academic work and other extra curricular activities. I made several very good friends during my stay in Göttingen. To name a few among them are Stephan, Fabian, Ingo, Florian, Ehsan, Elham, Navid, Mariya, Johannes, Davide and Salvatore. I spent a lot of time with them, discussing physics, politics, games, movies, etc during our extended coffee breaks, movie nights, journal clubs, barbecues and several other get-togethers. I would also like to thank my very dear friends Shubham and Debanjan, whom I know from my graduation days in Kolkata, for their continuous support throughout my stay in Göttingen.

Special thanks to my parents and sister for encouraging me to pursue a career in science and for standing with me during the good and bad times. Thanks to my dear friend Bochra for supporting and tolerating me during my doctoral studies.

Financial support from DFG-FOR1346 is sincerely acknowledged. 



\title{
Ebad Kamil
}

\author{
Hannoversche Str. 134 - Göttingen - Germany \\ (\$) +49 $17671617904 \bullet \bowtie$ kamilebad@gmail.com
}

\section{Education}

Georg-August Universität

Doctor of Philosophy, Computational Condensed Matter Physics

Thesis: Unbiased evaluation and development of

Green's-function-based density-matrix functionals

Supervisor: Prof. Thomas Pruschke (1959-2016) and Prof. Peter Blöchl

Indian Institute of Science Education and Research

BS-MS Dual degree

Thesis: Dephasing of a Qubit due to Quantum and Classical noise CGPA: $9.05 / 10$

St. Anthony College

All India Senior School Certificate Examination

Result: $87.6 \%$

Place and date of birth

- Barabanki, India. 08. December 1987

\section{Fellowships}

Deutsche Forschungsgemeinschaft (DFG) grant

Pursuing doctoral studies at Georg-August Universität

Deutscher Akademischer Austauschdienst (DAAD-WISE) fellowship

Intership at Institut für Theoretische Physik, Universität Augsburg

INSPIRE Fellowship

Pursuing undergraduate and graduate studies at IISER

\section{Teaching Experience}

○ George-August Universität

Teaching assistant for "Advanced Condensed Matter Physics"

Göttingen, Germany

Tutor for "Classical Field Theory"

- IISER Kolkata

Teaching assistant for "Advanced Quantum Mechanics"

\section{Publications and Conferences}

- E. Kamil, R. Schade, T. Pruschke and P. E. Blöchl. Reduced density-matrix functionals applied to the hubbard dimer. Phys. Rev. B 93, 085141, 2016.

- E. Kamil, T. Pruschke and P. E. Blöchl. Resolvent approach for the evaluation of the reduced density-matrix functional of correlated electron systems. AIP Conf. Proc. 1550, 253 (2013).

- E. Kamil and S. Dattagupta. Dephasing of a qubit due to quantum and classical noise. Pramana, Vol. 79, Issue 3, pp 357-376 (2012)

- Presented scientific work at international conferences in Germany, France, Italy and Slovenia.

\section{Skills}

Platform: Unix, Windows

Language: $\mathrm{C}++$, Python, Fortran, Bash (scripting). LaTeX for high quality typesetting.

Numerical libraries for scientific computing: GSL, NLopt, BLAS/LAPACK, NumPy/SciPy.

Softwares: Mathematica, Gnuplot 